Supporting Information for

\title{
Mechanisms for Formation of Diazocinones, Pyridazines, and Pyrazolines from Tetrazines-Oxyanion-Accelerated Pericyclic Cascades?
}

\author{
Michael W. Lodewyk, Mark J. Kurth, and Dean J. Tantillo* \\ Department of Chemistry \\ University of California, Davis \\ One Shields Avenue \\ Davis, CA 95616
}

\section{Table of Contents}

Gaussian03 Full Reference............................................S1

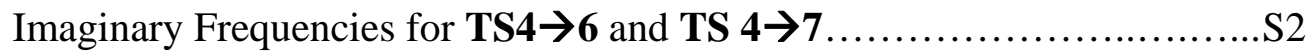

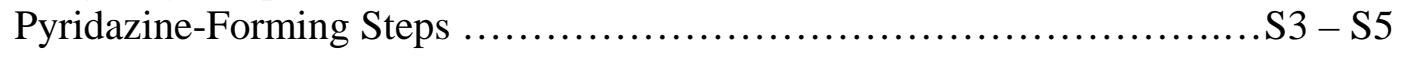

Relaxed PES Scan of C-C (enolate-tetrazine) distance in 1+2 Complex

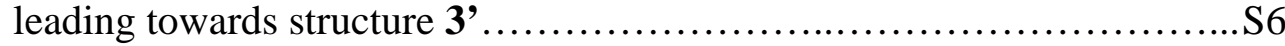

Relaxed Potential Energy Surface Scan of C3-C4 distance in 3'............ S7

Electrostatic potential maps for model systems lacking the phenyl rings.... S7

Coordinates and Energies....................................... S8 - S97

IRC Plots........................................................... S98 104 


\section{Gaussian03 Full Reference}

\section{GAUSSIAN03, Revision D.01}

M. J. Frisch, G. W. Trucks, H. B. Schlegel, G. E. Scuseria,

M. A. Robb, J. R. Cheeseman, J. A. Montgomery, Jr., T. Vreven, K. N. Kudin, J. C. Burant, J. M. Millam, S. S. Iyengar, J. Tomasi, V. Barone, B. Mennucci, M. Cossi, G. Scalmani, N. Rega,

G. A. Petersson, H. Nakatsuji, M. Hada, M. Ehara, K. Toyota, R. Fukuda, J. Hasegawa, M. Ishida, T. Nakajima, Y. Honda, O. Kitao, H. Nakai, M. Klene, X. Li, J. E. Knox, H. P. Hratchian, J. B. Cross, V. Bakken, C. Adamo, J. Jaramillo, R. Gomperts, R. E. Stratmann, O. Yazyev, A. J. Austin, R. Cammi, C. Pomelli, J. W. Ochterski, P. Y. Ayala, K. Morokuma, G. A. Voth, P. Salvador, J. J. Dannenberg, V. G. Zakrzewski, S. Dapprich, A. D. Daniels, M. C. Strain, O. Farkas, D. K. Malick, A. D. Rabuck, K. Raghavachari, J. B. Foresman, J. V. Ortiz, Q. Cui, A. G. Baboul, S. Clifford, J. Cioslowski, B. B. Stefanov, G. Liu, A. Liashenko, P. Piskorz, I. Komaromi, R. L. Martin, D. J. Fox, T. Keith, M. A. Al-Laham, C. Y. Peng, A. Nanayakkara, M. Challacombe, P. M. W. Gill, B. Johnson, W. Chen, M. W. Wong, C. Gonzalez, and J. A. Pople, Gaussian, Inc., Wallingford CT, 2004. 


\section{Imaginary Frequencies for TS4 $\rightarrow 6$ and TS $4 \rightarrow 7$ (see footnote 20)}

As mentioned in footnote 20, the assignment of disrotatory or conrotatory descriptors to the motion of the imaginary frequencies in TS4 $\rightarrow \mathbf{6}$ and TS4 $\rightarrow \mathbf{7}$ is somewhat ambiguous. While in TS4 $\rightarrow \mathbf{6}$, the hydrogen atom on C7 clearly rotates outwards and in TS4 $\rightarrow 7$ the same hydrogen atom clearly rotates inwards, the motion of the oxygen atom is much less clear, and appears to correspond mainly to planarization of the carbon center in both structures. Therefore, our assignment of a disrotatory product and a conrotatory product is based on the structures which result from forward IRC calculations, and subsequent optimization of these end points. From this perspective, the oxygen is ultimately rotated outwards in both products, and thus TS4 $\rightarrow \mathbf{6}$, with the H rotated outwards can be said to correspond to a disrotatory product while TS4 $\rightarrow$, with the $\mathrm{H}$ rotated inwards can be said to correspond to a conrotatory product. Note that this situation bears a connection to systems which have been described by Borden, et al. as “monorotatory”, see J. Chem. Soc., Perkin Trans. 2, 1999, 2339-2347.

Shown below are images containing frame shots of the equilibrium (center images) and extreme positions of the animated frequencies for both structures. Also included along with this document are animated .gif files for the imaginary frequency of each structure.

\section{TS4 $\rightarrow 6$}
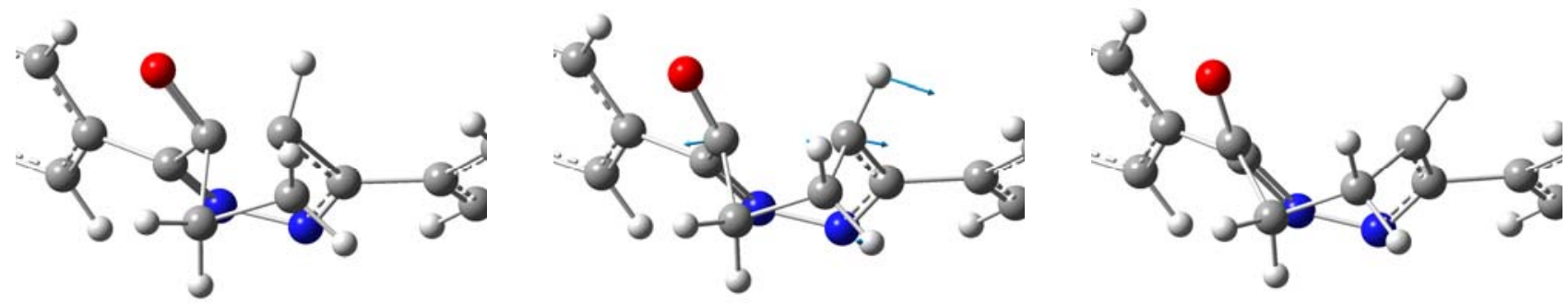

TS4 $\rightarrow 7$
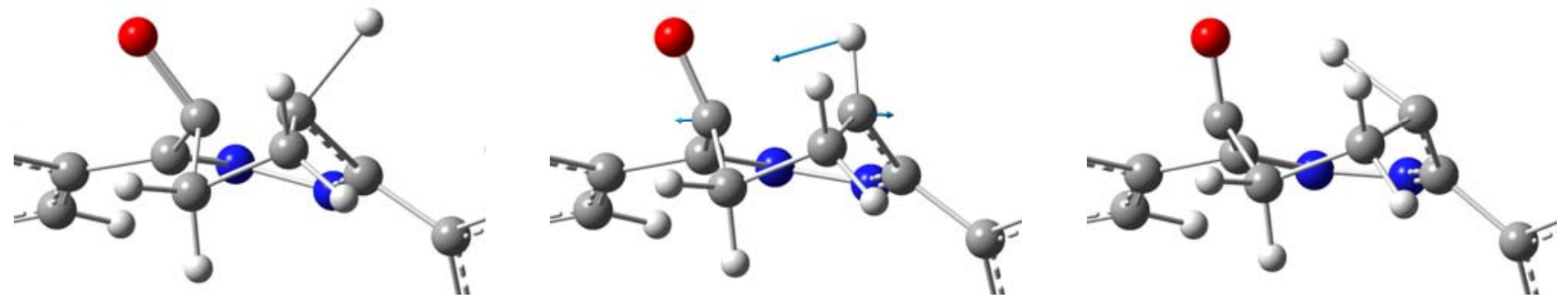


\section{Pyridazine-Forming Steps (see footnote 26)}

As proposed in Scheme 2, the aromatization pathway likely occurs via a protonation-elimination pathway involving an external proton source and an external base. This involvement of external species presents a challenge in examining the energetics of these processes with computations. Here, we make an attempt to examine plausible steps in a discrete sense.

The first step, protonation of the oxyanion to give $\mathbf{7}$ is likely to occur intermolecularly, with methanol as the obvious proton source. At this point, a syn-elimination is required to form the corresponding pyridazine. An intramolecular, concerted version of this process would almost certainly face an exceedingly high reaction barrier, and therefore we hypothesize that the elimination may occur via a two-step, E1cb-type mechanism, where the hydrogen at C7 is deprotonated to form a delocalized carbanion prior to elimination of hydroxide. The E1cb mechanism is frequently invoked when the possibility exists for the formation of a stabilized carbanion and when a relatively poor leaving group must be eliminated. For example, the final elimination step in the common aldol condensation reaction is often formulated in this way, and this same mechanism was proposed very recently by Domingo et al, for systems closely related to these (see reference $2 \mathrm{f}$ ). This process is detailed in the scheme below.

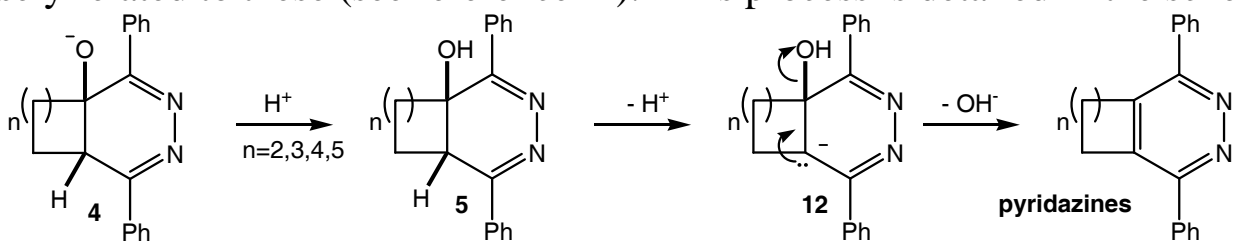

In order to test the feasibility of this hypothesis, we computed the $\mathbf{1 2} \rightarrow$ pyridazine step for the system derived from cyclopentanone, as well as the system derived from cyclobutanone for comparison. As shown in the figure below, we were able to find minima for the delocalized carbanions, and transitionstate structures for the elimination of hydroxide ion. Product complexes with the hydroxide ion associated with aromatic and aliphatic hydrogen atoms in the systems were also found (vida infra), but these gas-phase structures are likely not meaningful representations of the solution phase where the hydroxide ion is expected to be solvated. 

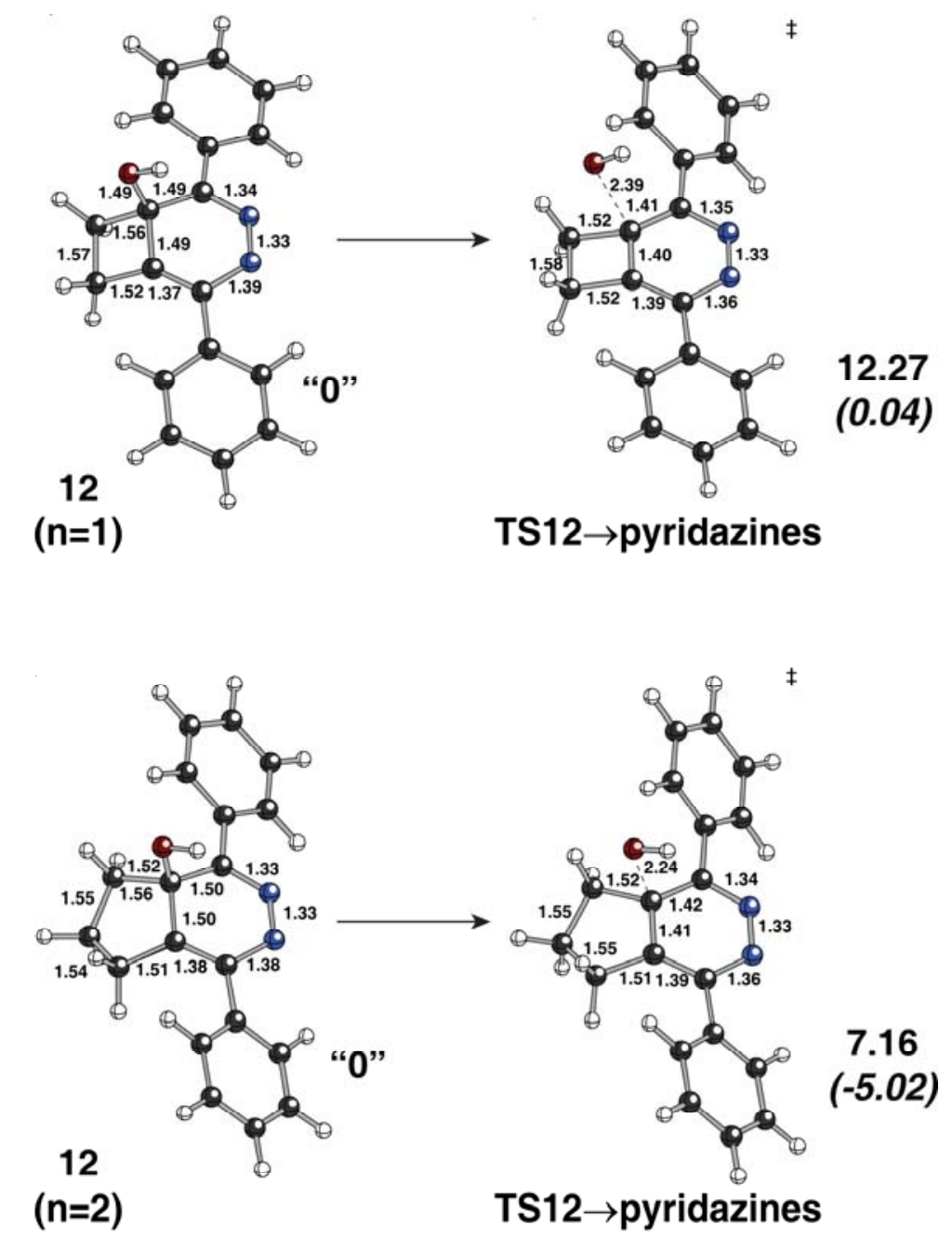

Computed geometries and energies (B3LYP/6-31+G(d,p)) for stationary point structures in the proposed E1cb transformation leading to pyridazines $(n=1$ and $n=2)$. Energies are in $\mathrm{kcal} / \mathrm{mol}$; regular text shows gas-phase free energy values, italicized text in parentheses shows solvent single-point electronic energy values (see methods). Selected distances are in $\AA$.

The scheme below summarizes the energies associated with the potential energy surfaces for the cyclobutanone-derived $(n=1)$ and cyclopentanone-derived $(n=2)$ systems in the regions involved in the ring-opening and aromatization processes. Following the $\mathrm{n}=1$ pathway, we see that $\mathbf{4}$ can either undergo exothermic ring-opening with a barrier of $10 \mathrm{kcal} / \mathrm{mol}$, or can isomerize via intermolecular proton transfer to 12. At this point, it faces a barrier ranging from $\sim 4-12 \mathrm{kcal} / \mathrm{mol}$ towards the fused pyridazine product. In comparison, the $\mathrm{n}=2$ system can undergo ring-opening with a somewhat higher barrier of $\sim 11-22 \mathrm{kcal} / \mathrm{mol}$, but can isomerize to $\mathbf{1 2}$ in a process that is significantly more exergonic than for the $\mathrm{n}=1$ system. From there, the transformation to a pyridazine is essentially barrierless. Beyond the two transition-state structures, intermediate $\mathbf{6}$ leading towards the diazocinone products represents a more exergonic product for $n=1$ than it does for $n=2$. For the fused pyridazines, the $n=1$ structure is slightly exergonic, while the $\mathrm{n}=2$ product is overall exergonic by $\sim 14-19 \mathrm{kcal} / \mathrm{mol}$. Both values likely underestimate the true exergonicity since the structures involve a non-solvated hydroxide ion. Thus, the 
theoretical evidence points to an easier and more exergonic ring-opening for the $n=1$ system than for the $\mathrm{n}=2$ system.

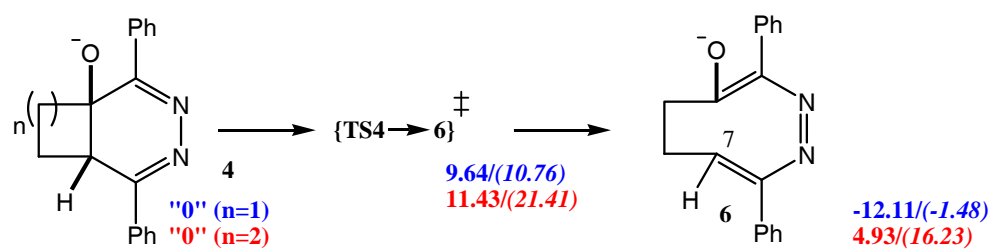

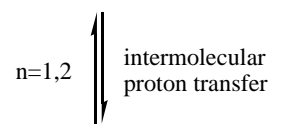

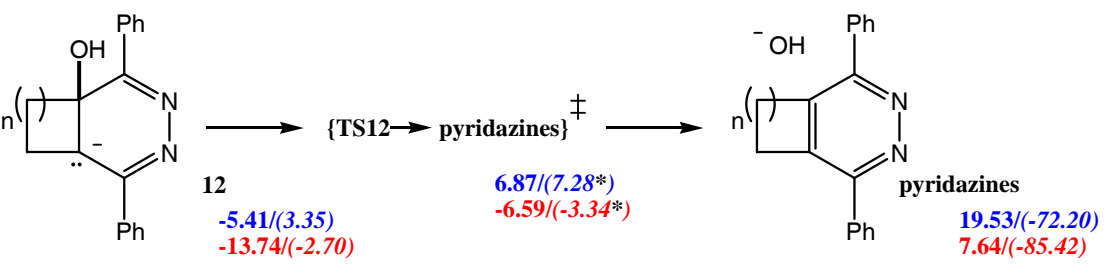

Energies (B3LYP/6-31+G(d,p)) are in kcal/mol; regular text shows gas-phase free energy values, italicized text in parentheses shows optimized solvent free energy values (*Values for the TS12 $\rightarrow$ pyridazine transitions structures are derived from calculations utilizing ua0 radii instead of uaks, and are relative to structures 4 computed with the same method). Selected distances are in Å. Note that for the pyridazines, the structure includes an associated hydroxide ion (computed as an isolated species), which likely artificially raises the energy compared to the expected solution phase behavior where the hydroxide ion would be dissociated and solvated. 
Relaxed Potential Energy Surface Scan of C-C (enolate-tetrazine) distance in 1+2 Complex leading towards structure 3'

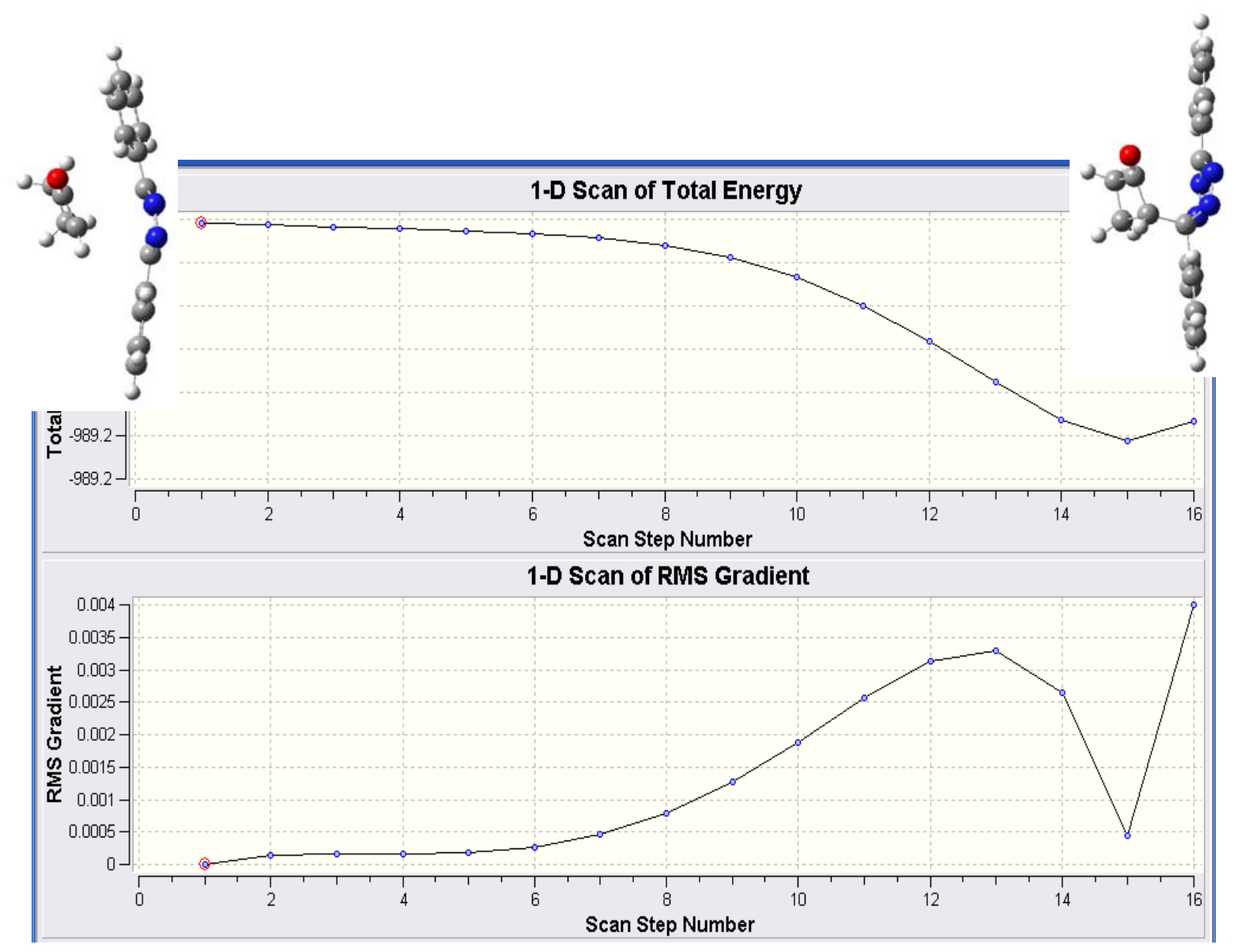

The C7-C8 distance here was scanned from $3.01 \AA$ to $1.51 \AA$ in $0.1 \AA$ increments. 


\section{Relaxed Potential Energy Surface Scan of C3-C4 distance in 3' (see footnote 17)}

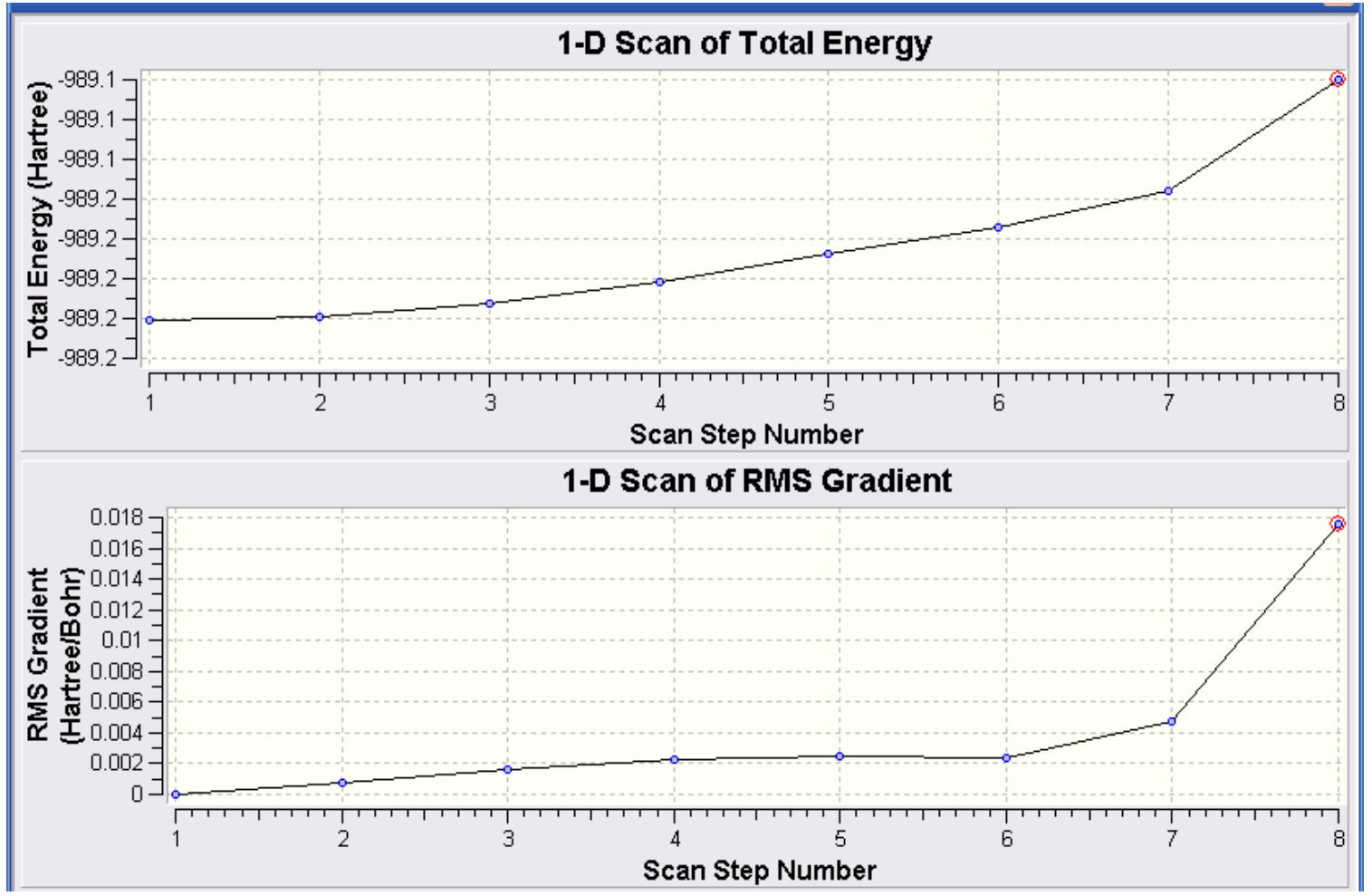

The C3-C4 distance here was scanned from $2.79 \AA$ to $1.39 \AA$ in $0.2 \AA$ increments.

Electrostatic potential maps for model systems lacking the phenyl rings

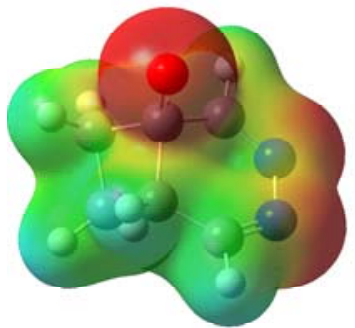

model of 4

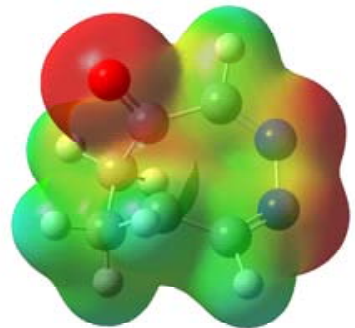

model of TS $4 \rightarrow 6$

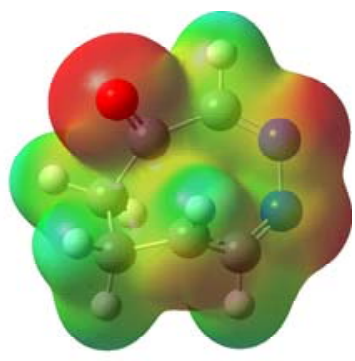

model of TS4 $\rightarrow 7$ 


\section{Coordinates and Energies}

Coordinates and energies correspond to gas phase optimizations, except where noted.

\section{1+2 complex (n=1, Figure 1)}

$\mathrm{HF}=-989.1655142$ hartrees $(-620711.251815642 \mathrm{kcal} / \mathrm{mol})$

Imaginary Frequencies: $1(-31.9351$ 1/cm)

Zero-point correction $=0.290735$ (Hartree/Particle)

Temperature 298.150 Kelvin. Pressure 1.00000 Atm.

Sum of electronic and thermal Free Energies $=\mathbf{- 9 8 8 . 9 2 3 8 3 8 ~ h a r t r e e s ~}(-620559.59758338 \mathrm{kcal} / \mathrm{mol})$

Solvent Single Point: HF = $\mathbf{- 9 8 9 . 2 4 9 1 0 2 5}$ hartrees $(-620763.7043 \mathrm{kcal} / \mathrm{mol})$

Coordinates (from last standard orientation):

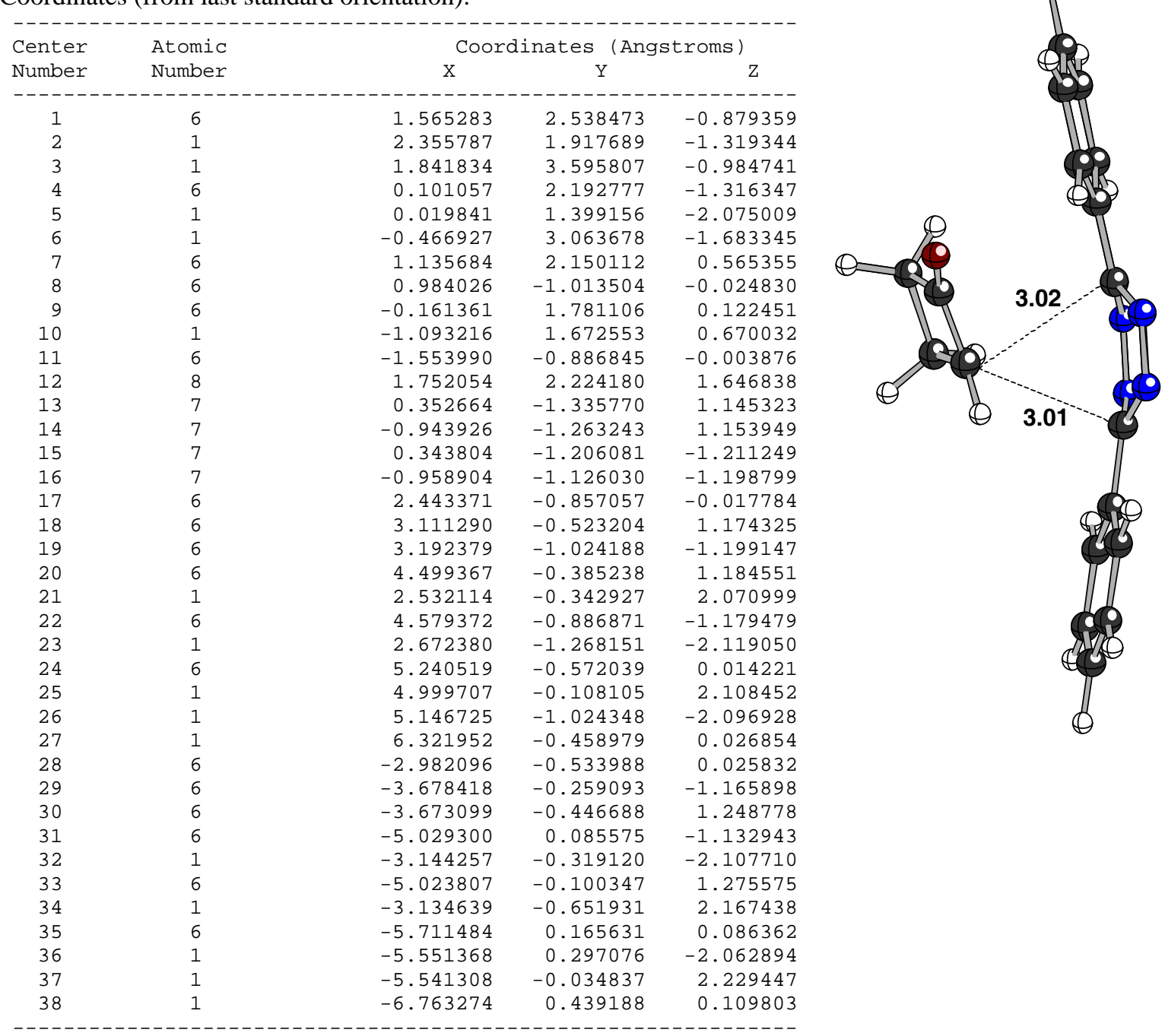




\section{3' (n=1, Figure 1)}

$\mathrm{HF}=-989.1906031$ hartrees $(-620726.995351281 \mathrm{kcal} / \mathrm{mol})$

Imaginary Frequencies: none found

Zero-point correction $=0.293790$ (Hartree/Particle)

Temperature 298.150 Kelvin. Pressure 1.00000 Atm.

Sum of electronic and thermal Free Energies = -988.944525 hartrees $\mathbf{( - 6 2 0 5 7 2 . 5 7 8 8 8 2 7 5} \mathbf{~ k c a l} / \mathbf{m o l})$

Solvent Single Point: HF $=\mathbf{- 9 8 9 . 2 7 5 9 4 6 7 ~ h a r t r e e s ~}(-620780.5493 \mathrm{kcal} / \mathrm{mol})$

Coordinates (from last standard orientation):

\begin{tabular}{|c|c|c|c|c|}
\hline \multirow{2}{*}{$\begin{array}{l}\text { Center } \\
\text { Number }\end{array}$} & \multirow{2}{*}{$\begin{array}{l}\text { Atomic } \\
\text { Number }\end{array}$} & \multicolumn{3}{|c|}{ Coordinates (Angstroms) } \\
\hline & & $\mathrm{X}$ & $\mathrm{Y}$ & Z \\
\hline \multicolumn{5}{|c|}{ 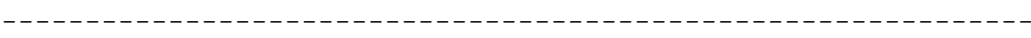 } \\
\hline 1 & 6 & 0.605579 & 2.613045 & -0.466499 \\
\hline 2 & 1 & 1.277264 & 2.155364 & -1.198654 \\
\hline 3 & 1 & 0.901759 & 3.655043 & -0.302806 \\
\hline 4 & 6 & -0.903081 & 2.319384 & -0.731862 \\
\hline 5 & 1 & -1.124690 & 1.894851 & -1.712912 \\
\hline 6 & 1 & -1.548959 & 3.182984 & -0.546752 \\
\hline 7 & 6 & 0.463064 & 1.787885 & ๑. 822317 \\
\hline 8 & 6 & 1.265063 & -0.699731 & -0.151646 \\
\hline 9 & 6 & $-\odot .932589$ & 1.274362 & $\odot .433850$ \\
\hline 10 & 1 & -1.639886 & 1.494801 & 1. 239716 \\
\hline 11 & 6 & -1.240100 & $-\odot .231879$ & -0.001028 \\
\hline 12 & 8 & 1.046396 & 1.853554 & 1.887046 \\
\hline 13 & 7 & $\odot .594279$ & -0.634623 & -1.339023 \\
\hline 14 & 7 & -0.690101 & -0.552403 & -1.322787 \\
\hline 15 & 7 & -0.666134 & -1.169028 & 0.980366 \\
\hline 16 & 7 & ๑. 610640 & -1.244478 & ๑. 923943 \\
\hline 17 & 6 & 2.729077 & -0.651333 & -0.147981 \\
\hline 18 & 6 & 3.459611 & -0.424793 & -1.333848 \\
\hline 19 & 6 & 3.449759 & -0.786952 & 1.056725 \\
\hline 20 & 6 & 4.852755 & $-\odot .355135$ & -1.315055 \\
\hline 21 & 1 & 2. 914531 & -0.318485 & -2.266136 \\
\hline 22 & 6 & 4.842553 & -0.718633 & 1.068627 \\
\hline 23 & 1 & 2.895485 & -0.940601 & 1.976099 \\
\hline 24 & 6 & 5.558703 & $-\odot .504080$ & $-\odot .115174$ \\
\hline 25 & 1 & 5.391997 & $-\odot .184962$ & -2.244854 \\
\hline 26 & 1 & 5.373756 & $-\odot .825298$ & 2. 012094 \\
\hline 27 & 1 & 6.644448 & -0.446396 & -0.101848 \\
\hline 28 & 6 & -2.756733 & -0.393771 & -0.030398 \\
\hline 29 & 6 & -3.480965 & $-\odot .287353$ & -1.225746 \\
\hline 30 & 6 & -3.462085 & -0.619725 & 1.161740 \\
\hline 31 & 6 & -4.875893 & -0.392999 & -1.227784 \\
\hline 32 & 1 & -2.935816 & -0.144989 & -2.151922 \\
\hline 33 & 6 & -4.854523 & -0.730400 & 1. 160622 \\
\hline 34 & 1 & -2.903148 & -0.729715 & 2.085589 \\
\hline 35 & 6 & -5.570776 & -0.614271 & -0.035586 \\
\hline 36 & 1 & -5.419399 & -0.307673 & -2.166263 \\
\hline 37 & 1 & -5.380781 & $-\odot .912396$ & 2.095017 \\
\hline 38 & 1 & -6.655037 & -0.698942 & -0.037863 \\
\hline
\end{tabular}

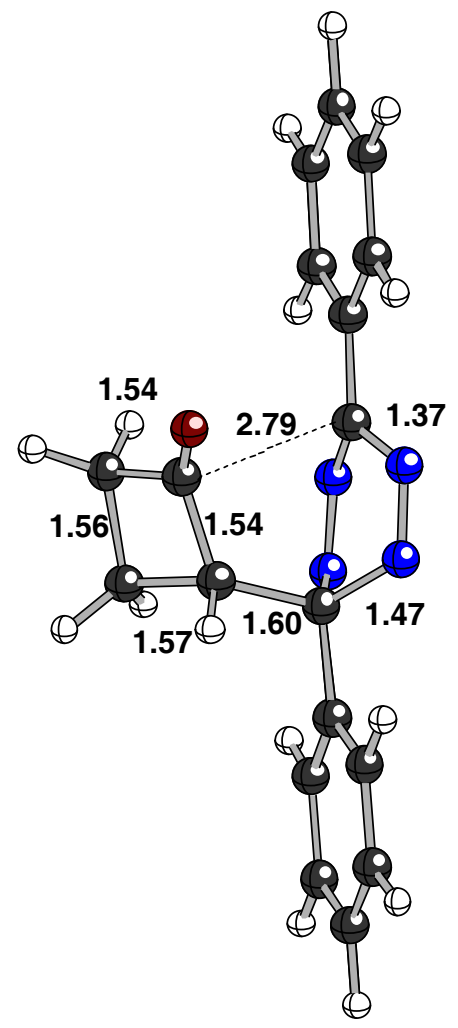




\section{TS3' $\rightarrow$ 4 (n=1, Figure 1)}

$\mathrm{HF}=-989.1512852$ hartrees $(-620702.322975852 \mathrm{kcal} / \mathrm{mol})$

Imaginary Frequencies: 1 (-459.9626 1/cm)

Zero-point correction $=0.291285$ (Hartree/Particle)

Temperature 298.150 Kelvin. Pressure 1.00000 Atm.

Sum of electronic and thermal Free Energies = -988.907283 hartrees (-620549.20915533 $\mathbf{k c a l} / \mathrm{mol})$

Solvent Single Point: HF = -989.2372322 hartrees $(-620756.2556 \mathrm{kcal} / \mathrm{mol})$

Coordinates (from last standard orientation):

\begin{tabular}{|c|c|c|c|c|}
\hline \multirow{2}{*}{$\begin{array}{l}\text { Center } \\
\text { Number }\end{array}$} & \multirow{2}{*}{$\begin{array}{l}\text { Atomic } \\
\text { Number }\end{array}$} & \multicolumn{3}{|c|}{ Coordinates (Angstroms) } \\
\hline & & $x$ & $\mathrm{Y}$ & Z \\
\hline (--- & & $--\cdots-\cdots$ & -------1 & ------- \\
\hline 1 & 6 & -0.888506 & 2.117658 & 1.333368 \\
\hline 2 & 1 & -1.421913 & 1.535521 & 2.092355 \\
\hline 3 & 1 & -1.336353 & 3.112132 & 1.251669 \\
\hline 4 & 6 & 0.656394 & 2.100651 & 1.456076 \\
\hline 5 & 1 & 1.044384 & 1.520131 & 2.298976 \\
\hline 6 & 1 & 1.117119 & 3.094620 & 1.474439 \\
\hline 7 & 6 & -0.776912 & 1.458405 & -0.104997 \\
\hline 8 & 6 & -1.205679 & -0.175673 & 0.034321 \\
\hline 9 & 6 & 0.823008 & 1.382594 & 0.078407 \\
\hline 10 & 1 & 1.306347 & 1.981502 & $-\odot .695599$ \\
\hline 11 & 6 & 1.393508 & $-\odot .012652$ & 0.152313 \\
\hline 12 & 8 & -1.284642 & 2.063243 & -1.120477 \\
\hline 13 & 7 & -0.547014 & -0.920338 & ๑. 985185 \\
\hline 14 & 7 & $\odot .760753$ & -0.896317 & 1.006454 \\
\hline 15 & 7 & 0.643828 & -0.608377 & -1.522884 \\
\hline 16 & 7 & -0.522905 & $-\odot .620190$ & -1.439151 \\
\hline 17 & 6 & -2.678217 & -0.435798 & -0.031405 \\
\hline 18 & 6 & -3.340341 & -1.121383 & 1.000871 \\
\hline 19 & 6 & -3.432378 & ๑. 051237 & -1.113545 \\
\hline 20 & 6 & -4.721363 & -1.324520 & ๑. 950425 \\
\hline 21 & 1 & -2.755047 & -1.495611 & 1.833598 \\
\hline 22 & 6 & -4.813681 & $-\odot .155409$ & -1.159854 \\
\hline 23 & 1 & -2.921280 & ๑. 613654 & -1.885877 \\
\hline 24 & 6 & -5.465843 & -0.844670 & -0.132647 \\
\hline 25 & 1 & -5.216096 & -1.859815 & 1.758142 \\
\hline 26 & 1 & -5.383705 & $\odot .230471$ & -2.002046 \\
\hline 27 & 1 & -6.541211 & -1.003959 & -0.173441 \\
\hline 28 & 6 & 2.861092 & -0.246034 & 0.019863 \\
\hline 29 & 6 & 3.388787 & -1.527986 & 0.267062 \\
\hline 30 & 6 & 3.752043 & ๑.768245 & -0.369439 \\
\hline 31 & 6 & 4.754180 & -1.780351 & 0.140673 \\
\hline 32 & 1 & 2.704883 & -2.314045 & 0.568016 \\
\hline 33 & 6 & 5.119936 & 0.514115 & -0.504157 \\
\hline 34 & 1 & 3.377978 & 1.767421 & -0.563383 \\
\hline 35 & 6 & 5.630873 & -0.760503 & -0.247664 \\
\hline 36 & 1 & 5.136491 & -2.778831 & 0.340256 \\
\hline 37 & 1 & 5.786505 & 1.317999 & -0.807463 \\
\hline 38 & 1 & 6.694982 & -0.958147 & -0.351076 \\
\hline
\end{tabular}

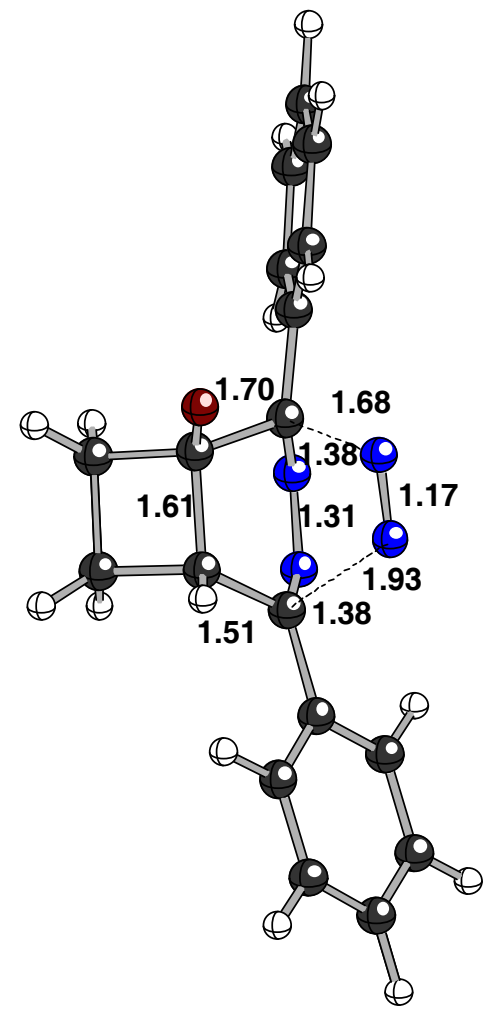




\section{$\underline{4(n=1, \text { Figures } 1 \text { and } 2 \text {, and pyridazine scheme and figure in } \mathrm{SI})}$}

(gas phase optimization)

$\mathrm{HF}=-879.6946968$ hartrees $(-552017.219188968 \mathrm{kcal} / \mathrm{mol})$

Imaginary Frequencies: none found

Zero-point correction $=0.284080$ (Hartree/Particle)

Temperature 298.150 Kelvin. Pressure 1.00000 Atm.

Sum of electronic and thermal Free Energies $=\mathbf{- 8 7 9 . 4 5 5 7 0 5}$ hartrees $(-551867.24944455 \mathrm{kcal} / \mathrm{mol})$

Solvent Single Point: HF = -879.7937401 hartrees $(-552079.3699 \mathrm{kcal} / \mathrm{mol})$

Coordinates (from last standard orientation):

\begin{tabular}{|c|c|c|c|c|}
\hline \multirow{2}{*}{$\begin{array}{l}\text { Center } \\
\text { Number }\end{array}$} & \multirow{2}{*}{$\begin{array}{l}\text { Atomic } \\
\text { Number }\end{array}$} & \multicolumn{3}{|c|}{ Coordinates (Angstroms) } \\
\hline & & $x$ & $\mathrm{Y}^{\prime}$ & Z \\
\hline 1 & 6 & $-\odot .998316$ & 2.203331 & 0.780429 \\
\hline 2 & 1 & -1.941522 & 2.113645 & 1.332891 \\
\hline 3 & 1 & -0.910765 & 3.217228 & 0.372402 \\
\hline 4 & 6 & 0.303416 & 1.743616 & 1.476141 \\
\hline 5 & 1 & 0.127733 & 0.946083 & 2.205791 \\
\hline 6 & 1 & 0.934395 & 2.506910 & 1.946758 \\
\hline 7 & 6 & $-\odot .698835$ & 1.184889 & $-\odot .401277$ \\
\hline 8 & 6 & -1.274914 & -0.204326 & $-\odot .079687$ \\
\hline 9 & 6 & 0.815426 & 1.171426 & $\odot .117966$ \\
\hline 10 & 1 & 1.367009 & 1.934768 & -0.440114 \\
\hline 11 & 6 & 1.479012 & -0.154360 & 0.075160 \\
\hline 12 & 8 & $-\odot .966799$ & 1.486027 & -1.661168 \\
\hline 13 & 7 & -0.580624 & -1.293698 & $\odot .114156$ \\
\hline 14 & 7 & 0.814795 & -1.274119 & 0.118286 \\
\hline 15 & 6 & 2.959368 & -0.279821 & -0.023468 \\
\hline 16 & 6 & 3.561978 & -1.522346 & -0.310271 \\
\hline 17 & 6 & 3.802774 & 0.827205 & 0.182421 \\
\hline 18 & 6 & 4.946932 & -1.648098 & -0.384439 \\
\hline 19 & 1 & 2.911481 & -2.375235 & -0.469469 \\
\hline 20 & 6 & 5.193119 & 0.701112 & 0.107213 \\
\hline 21 & 1 & 3.368539 & 1.795061 & 0.410859 \\
\hline 22 & 6 & 5.774738 & -0.536326 & -0.176743 \\
\hline 23 & 1 & 5.386868 & -2.616692 & -0.611212 \\
\hline 24 & 1 & 5.820733 & 1.573780 & 0.272243 \\
\hline 25 & 1 & 6.855738 & -0.635331 & -0.238310 \\
\hline 26 & 6 & -2.753151 & -0.389630 & $-\odot .088659$ \\
\hline 27 & 6 & -3.328189 & -1.442367 & 0.650711 \\
\hline 28 & 6 & -3.612829 & 0.488715 & -0.777341 \\
\hline 29 & 6 & -4.712683 & -1.609740 & $\odot .705982$ \\
\hline 30 & 1 & -2.665560 & -2.122883 & 1.174810 \\
\hline 31 & 6 & -4.997848 & 0.320576 & -0.714102 \\
\hline 32 & 1 & -3.154227 & 1.271050 & -1.375335 \\
\hline 33 & 6 & -5.558498 & -0.727418 & $\odot .024651$ \\
\hline 34 & 1 & -5.132719 & -2.429603 & 1.285107 \\
\hline 35 & 1 & -5.643729 & 1.006632 & -1.258070 \\
\hline 36 & 1 & -6.637774 & -0.857526 & $\odot .065775$ \\
\hline
\end{tabular}

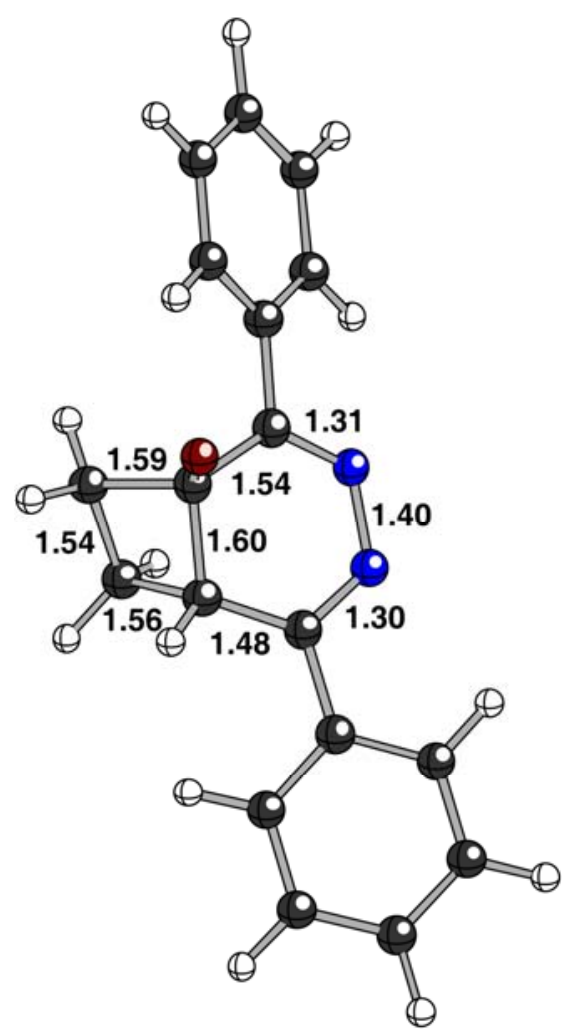




\section{4 (n=1, Figures 1 and 2 , and pyridazine scheme and figure in SI)}

(solvent optimization, methanol, uaks radii)

$\mathrm{HF}=-879.7964677$ hartrees $(-552081.081446427 \mathrm{kcal} / \mathrm{mol})$

Imaginary Frequencies: none found

Zero-point correction $=0.284840$ (Hartree/Particle)

Temperature 298.150 Kelvin. Pressure 1.00000 Atm.

Sum of electronic and thermal Free Energies $=-879.556503$ hartrees $(-551930.50119753 \mathrm{kcal} / \mathrm{mol})$

Coordinates (from last standard orientation):

\begin{tabular}{|c|c|c|c|c|}
\hline \multirow{2}{*}{$\begin{array}{l}\text { Center } \\
\text { Number }\end{array}$} & \multirow{2}{*}{$\begin{array}{l}\text { Atomic } \\
\text { Number }\end{array}$} & \multicolumn{3}{|c|}{ Coordinates (Angstroms) } \\
\hline & & $\mathrm{x}$ & $\mathrm{Y}$ & z \\
\hline 1 & 6 & 1.011477 & 2.184779 & -0.625505 \\
\hline 2 & 1 & 1.982941 & 2.129325 & -1.127335 \\
\hline 3 & 1 & $\odot .876759$ & 3.190754 & -0.209914 \\
\hline 4 & 6 & -0.241392 & 1.720280 & -1.410117 \\
\hline 5 & 1 & $-\odot .009799$ & $\odot .941475$ & -2.144656 \\
\hline 6 & 1 & -0.857699 & 2.485622 & -1.891462 \\
\hline 7 & 6 & 0.667422 & 1.127501 & 0.493165 \\
\hline 8 & 6 & 1.284822 & $-\odot .235837$ & $\odot .141721$ \\
\hline 9 & 6 & -0.800751 & 1.125611 & -0.079805 \\
\hline 10 & 1 & -1.379990 & 1.889615 & 0.454111 \\
\hline 11 & 6 & -1.485012 & $-\odot .19659 \odot$ & -0.051001 \\
\hline 12 & 8 & 0.855030 & 1.407922 & 1.811786 \\
\hline 13 & 7 & 0.589875 & -1.314288 & -0.078604 \\
\hline 14 & 7 & -0.817310 & -1.312854 & -0.045237 \\
\hline 15 & 6 & -2.967786 & $-\odot .292090$ & 0.006136 \\
\hline 16 & 6 & -3.608168 & -1.476464 & 0.422115 \\
\hline 17 & 6 & -3.767672 & 0.802544 & -0.370256 \\
\hline 18 & 6 & -4.998978 & -1.561090 & 0.457912 \\
\hline 19 & 1 & -3.001258 & -2.324571 & 0.722174 \\
\hline 20 & 6 & -5.162341 & 0.714661 & -0.341835 \\
\hline 21 & 1 & -3.304074 & 1.728352 & -0.697649 \\
\hline 22 & 6 & -5.783849 & $-\odot .465956$ & ๑. 073679 \\
\hline 23 & 1 & -5.474095 & -2.481158 & 0.790554 \\
\hline 24 & 1 & -5.761089 & 1.570609 & -0.644081 \\
\hline 25 & 1 & -6.868846 & -0.533155 & 0.102172 \\
\hline 26 & 6 & 2.766576 & $-\odot .391396$ & 0.077548 \\
\hline 27 & 6 & 3.337201 & -1.475623 & -0.619323 \\
\hline 28 & 6 & 3.634163 & 0.550501 & 0.662244 \\
\hline 29 & 6 & 4.721761 & -1.617348 & $-\odot .719554$ \\
\hline 30 & 1 & 2.681682 & -2.201790 & -1.088742 \\
\hline 31 & 6 & 5.020132 & 0.406622 & 0.563947 \\
\hline 32 & 1 & 3.208964 & 1.380874 & 1.215059 \\
\hline 33 & 6 & 5.572272 & -0.677479 & -0.125957 \\
\hline 34 & 1 & 5.137619 & -2.460195 & -1.267161 \\
\hline 35 & 1 & 5.669932 & 1.142925 & 1.031612 \\
\hline 36 & 1 & 6.651484 & -0.787830 & $-\odot .202780$ \\
\hline
\end{tabular}

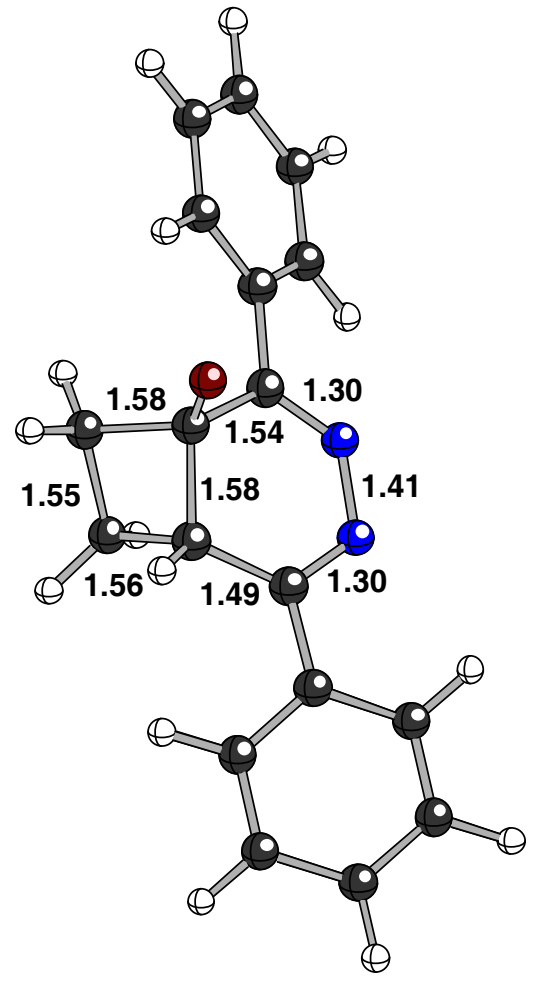




\section{4 (n=1, Figures 1 and 2 , and pyridazine scheme and figure in SI)}

(solvent optimization, methanol, ua0 radii)

$\mathrm{HF}=-879.7904059$ hartrees $(-552077.277606309 \mathrm{kcal} / \mathrm{mol})$

Imaginary Frequencies: none found

Zero-point correction $=0.283702$ (Hartree/Particle)

Temperature 298.150 Kelvin. Pressure 1.00000 Atm.

Sum of electronic and thermal Free Energies = -879.551618 hartrees $(-551927.43581118 \mathrm{kcal} / \mathrm{mol})$

Coordinates (from last standard orientation):

\begin{tabular}{|c|c|c|c|c|}
\hline \multirow{2}{*}{$\begin{array}{l}\text { Center } \\
\text { Number }\end{array}$} & \multirow{2}{*}{$\begin{array}{l}\text { Atomic } \\
\text { Number }\end{array}$} & \multicolumn{3}{|c|}{ Coordinates (Angstroms) } \\
\hline & & $\mathrm{x}$ & $\mathrm{Y}$ & Z \\
\hline 1 & 6 & 1.010133 & 2.186433 & -0.647507 \\
\hline 2 & 1 & 1.973236 & 2.116415 & -1.164391 \\
\hline 3 & 1 & $\odot .898649$ & 3.193176 & -0.227601 \\
\hline 4 & 6 & -0.260430 & 1.740747 & -1.413141 \\
\hline 5 & 1 & -0.049457 & 0.972035 & -2.163827 \\
\hline 6 & 1 & -0.879667 & 2.516952 & -1.872298 \\
\hline 7 & 6 & 0.671771 & 1.131798 & 0.477236 \\
\hline 8 & 6 & 1.282974 & -0.232692 & 0.122695 \\
\hline 9 & 6 & -0.805941 & 1.130555 & $-\odot .084076$ \\
\hline 10 & 1 & -1.386038 & 1.883526 & 0.461332 \\
\hline 11 & 6 & -1.484864 & -0.193317 & -0.066458 \\
\hline 12 & 8 & 0.879402 & 1.399405 & 1.785006 \\
\hline 13 & 7 & 0.590717 & -1.315158 & -0.095434 \\
\hline 14 & 7 & -0.816657 & -1.310099 & -0.078504 \\
\hline 15 & 6 & -2.967626 & $-\odot .292875$ & 0.005471 \\
\hline 16 & 6 & -3.600557 & -1.475769 & 0.438592 \\
\hline 17 & 6 & -3.775607 & 0.797159 & -0.369497 \\
\hline 18 & 6 & -4.991415 & -1.565498 & 0.486547 \\
\hline 19 & 1 & -2.988851 & -2.320419 & $\odot .743557$ \\
\hline 20 & 6 & -5.170153 & 0.704482 & -0.327802 \\
\hline 21 & 1 & -3.320160 & 1.724763 & -0.705707 \\
\hline 22 & 6 & -5.784155 & -0.476174 & 0.100463 \\
\hline 23 & 1 & -5.460672 & -2.486137 & 0.831423 \\
\hline 24 & 1 & -5.775399 & 1.558046 & -0.630159 \\
\hline 25 & 1 & -6.870388 & -0.547269 & 0.138578 \\
\hline 26 & 6 & 2.765433 & $-0.39 \odot 472$ & ๑.075588 \\
\hline 27 & 6 & 3.342070 & -1.416717 & -0.700365 \\
\hline 28 & 6 & 3.627641 & 0.494944 & 0.751436 \\
\hline 29 & 6 & 4.728406 & -1.560878 & -0.786375 \\
\hline 30 & 1 & 2.691647 & -2.095311 & -1.245657 \\
\hline 31 & 6 & 5.014754 & 0.348518 & 0.665982 \\
\hline 32 & 1 & 3.190348 & 1.280390 & 1.358897 \\
\hline 33 & 6 & 5.573670 & -0.680642 & -0.100291 \\
\hline 34 & 1 & 5.149743 & -2.358931 & -1.396738 \\
\hline 35 & 1 & 5.661607 & 1.039927 & 1.204958 \\
\hline 36 & 1 & 6.655140 & -0.793203 & -0.165851 \\
\hline
\end{tabular}

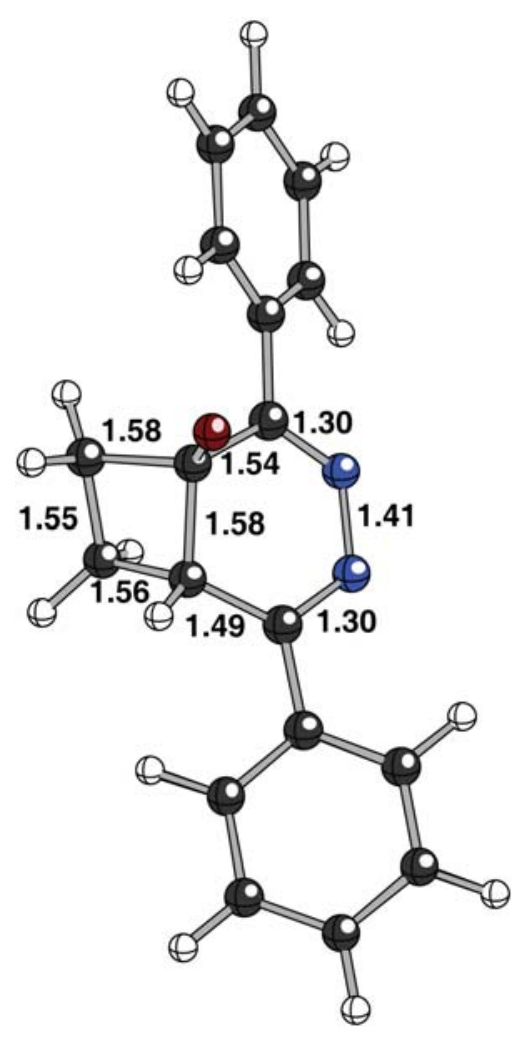




\section{$\mathbf{N}_{2}$ (Figure 1)}

$\mathrm{HF}=-109.5297794$ hartrees $(-68731.031871294 \mathrm{kcal} / \mathrm{mol})$

Imaginary Frequencies: none found

Zero-point correction $=0.005592$ (Hartree/Particle)

Temperature 298.150 Kelvin. Pressure 1.00000 Atm.

Sum of electronic and thermal Free Energies $=\mathbf{- 1 0 9 . 5 4 2 6 3 6}$ hartrees $(-\mathbf{6 8 7 3 9 . 0 9 9 5 1 6 3 6 ~ k c a l} / \mathbf{m o l})$

Solvent Single Point: HF $=\mathbf{- 1 0 9 . 5 3 0 5 9 0 6}$ hartrees $(-68731.54091 \mathrm{kcal} / \mathrm{mol})$

Coordinates (from last standard orientation):

\begin{tabular}{|c|c|c|c|c|}
\hline Center & Atomic & \multicolumn{3}{|c|}{ Coordinates (Angstroms) } \\
\hline Number & Number & $\mathrm{x}$ & $\mathrm{Y}$ & Z \\
\hline 1 & 7 & ๑. .००००० & 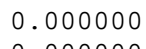 & 0.552563 \\
\hline 2 & 7 & $\odot .00000 \odot$ & $\odot .0000 \odot \odot$ & -0.552563 \\
\hline
\end{tabular}

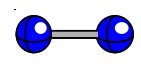


TS4 $\rightarrow \mathbf{6}(\mathrm{n}=1$, Figure 3 and pyridazine figure and scheme in SI)

$\mathrm{HF}=-879.6776885$ hartrees $(-552006.546310635 \mathrm{kcal} / \mathrm{mol})$

Imaginary Frequencies: 1 (-288.2393 1/cm)

Zero-point correction $=0.282473$ (Hartree/Particle)

Temperature 298.150 Kelvin. Pressure 1.00000 Atm.

Sum of electronic and thermal Free Energies = -879.440350 hartrees $(-551857.6140285 \mathrm{kcal} / \mathrm{mol})$

Solvent Single Point: HF = -879.7648279 hartrees $(-552061.2272 \mathrm{kcal} / \mathrm{mol})$

Coordinates (from last standard orientation):

\begin{tabular}{|c|c|c|c|c|}
\hline \multirow{2}{*}{$\begin{array}{l}\text { Center } \\
\text { Number }\end{array}$} & \multirow{2}{*}{$\begin{array}{l}\text { Atomic } \\
\text { Number }\end{array}$} & \multicolumn{3}{|c|}{ Coordinates (Angstroms) } \\
\hline & & $\mathrm{X}$ & $\mathrm{Y}$ & Z \\
\hline \multicolumn{5}{|c|}{ 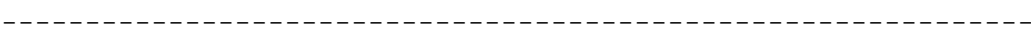 } \\
\hline 1 & 6 & -0.430325 & 2.303263 & 1.327847 \\
\hline 2 & 1 & -0.357697 & 1.639284 & 2.197120 \\
\hline 3 & 1 & -1.056497 & 3.168791 & 1.574565 \\
\hline 4 & 6 & ๑. 932077 & 2.589120 & 0.712941 \\
\hline 5 & 1 & 1.785832 & 2.664708 & 1.403211 \\
\hline 6 & 1 & ๑. 889738 & 3.504606 & 0.113499 \\
\hline 7 & 6 & -0.967509 & 1.561884 & 0.057375 \\
\hline 8 & 6 & -1.365188 & 0.130130 & 0.327286 \\
\hline 9 & 6 & $\odot .977464$ & 1.384894 & -0.256868 \\
\hline 10 & 1 & 1.332307 & 1.631780 & -1.262582 \\
\hline 11 & 6 & 1.465752 & ๑.129991 & ๑. 267450 \\
\hline 12 & 8 & -1.559740 & 2.268723 & -0.824685 \\
\hline 13 & 7 & $-\odot .596566$ & -0.766368 & ๑. 898141 \\
\hline 14 & 7 & 0.754607 & -0.713175 & 1.024729 \\
\hline 15 & 6 & 2.842185 & -0.340384 & -0.042129 \\
\hline 16 & 6 & 3.228551 & -1.677218 & ๑. 198581 \\
\hline 17 & 6 & 3.814000 & $\odot .534689$ & -0.569415 \\
\hline 18 & 6 & 4.526683 & -2.110053 & $-\odot .064579$ \\
\hline 19 & 1 & 2.480280 & -2.355135 & 0.594042 \\
\hline 20 & 6 & 5.109198 & ๑. 094515 & -0.853516 \\
\hline 21 & 1 & 3.551252 & 1.573337 & -0.743150 \\
\hline 22 & 6 & 5.476952 & -1.229760 & -0.599058 \\
\hline 23 & 1 & 4.797772 & -3.145219 & ๑.132171 \\
\hline 24 & 1 & 5.835898 & ๑. 792687 & -1.263008 \\
\hline 25 & 1 & 6.486255 & -1.572389 & -0.814324 \\
\hline 26 & 6 & -2.735314 & -0.362325 & -0.003837 \\
\hline 27 & 6 & -3.235223 & -1.515858 & ๑. 642912 \\
\hline 28 & 6 & -3.574387 & ๑. 286969 & -0.932697 \\
\hline 29 & 6 & -4.515234 & -1.995458 & 0.376844 \\
\hline 30 & 1 & -2.592134 & -2.019097 & 1.356086 \\
\hline 31 & 6 & -4.858707 & -0.202380 & -1.196069 \\
\hline 32 & 1 & -3.191288 & 1.171787 & -1.427103 \\
\hline 33 & 6 & -5.339822 & -1.342503 & -0.549493 \\
\hline 34 & 1 & -4.873917 & -2.882384 & ๑. 895323 \\
\hline 35 & 1 & -5.484536 & ๑. 315979 & -1.919646 \\
\hline 36 & 1 & -6.338541 & -1.718820 & -0.760264 \\
\hline
\end{tabular}

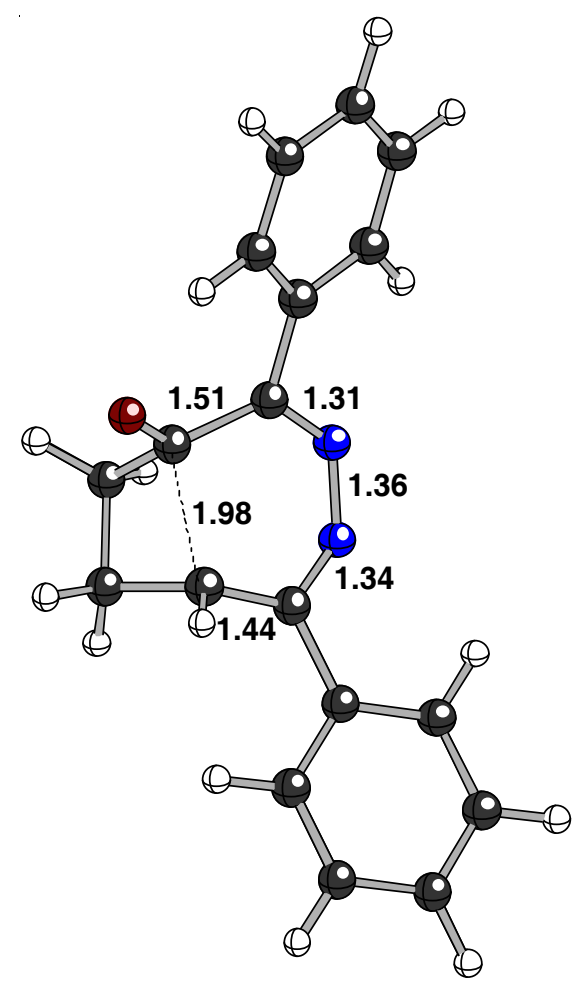


TS4 $\rightarrow 6(n=1$, Figure 2 and pyridazine figure and scheme in SI)

(solvent optimization, methanol, uaks radii)

$\mathrm{HF}=-879.7769758$ hartrees $(-552068.850084258 \mathrm{kcal} / \mathrm{mol})$

Imaginary Frequencies: 1 (-370.7367 1/cm)

Zero-point correction $=0.282639$ (Hartree/Particle)

Temperature 298.150 Kelvin. Pressure 1.00000 Atm.

Sum of electronic and thermal Free Energies = -879.539350 hartrees $(-551919.7375185 \mathrm{kcal} / \mathrm{mol})$

Coordinates (from last standard orientation):

\begin{tabular}{|c|c|c|c|c|}
\hline \multirow{2}{*}{$\begin{array}{l}\text { Center } \\
\text { Number }\end{array}$} & \multirow{2}{*}{$\begin{array}{l}\text { Atomic } \\
\text { Number }\end{array}$} & \multicolumn{3}{|c|}{ Coordinates (Angstroms) } \\
\hline & & $\mathrm{x}$ & $\mathrm{Y}$ & Z \\
\hline 1 & 6 & $\odot .989708$ & 2.425832 & -0.275345 \\
\hline 2 & 1 & 2.028057 & 2.544620 & -0.620505 \\
\hline 3 & 1 & $\odot .710558$ & 3.308401 & 0.314377 \\
\hline 4 & 6 & $-\odot .095613$ & 2.110179 & -1.312242 \\
\hline 5 & 1 & 0.268974 & 1.455287 & -2.110587 \\
\hline 6 & 1 & $-\odot .567093$ & 2.983201 & -1.781199 \\
\hline 7 & 6 & 0.734779 & 1.171495 & 0.597701 \\
\hline 8 & 6 & 1.242272 & $-\odot .077546$ & -0.114908 \\
\hline 9 & 6 & -1.026291 & 1.377354 & -0.346830 \\
\hline 10 & 1 & -1.648793 & 2.055707 & $\odot .241590$ \\
\hline 11 & 6 & -1.535021 & 0.066966 & -0.528844 \\
\hline 12 & 8 & 0.791079 & 1.208697 & 1.873893 \\
\hline 13 & 7 & 0.572666 & -0.920729 & -0.865337 \\
\hline 14 & 7 & -0.798759 & -0.963530 & -0.958983 \\
\hline 15 & 6 & -2.942074 & $-\odot .261074$ & -0.153298 \\
\hline 16 & 6 & -3.281729 & -1.538348 & ๑. 331591 \\
\hline 17 & 6 & -3.965504 & 0.696969 & -0.279002 \\
\hline 18 & 6 & -4.598620 & -1.847786 & 0.677194 \\
\hline 19 & 1 & -2.497851 & -2.281854 & $\odot .440012$ \\
\hline 20 & 6 & -5.283674 & ๑. 389921 & $\odot .068003$ \\
\hline 21 & 1 & -3.729810 & 1.684435 & -0.667254 \\
\hline 22 & 6 & -5.605659 & -0.884098 & 0.547809 \\
\hline 23 & 1 & -4.838880 & -2.838832 & 1.056128 \\
\hline 24 & 1 & -6.060241 & 1.143467 & -0.043098 \\
\hline 25 & 1 & -6.631298 & -1.123825 & 0.819261 \\
\hline 26 & 6 & 2.698565 & $-\odot .380567$ & 0.030927 \\
\hline 27 & 6 & 3.375732 & -1.074353 & -0.996962 \\
\hline 28 & 6 & 3.457656 & 0.040810 & 1.141975 \\
\hline 29 & 6 & 4.741485 & -1.343300 & -0.911964 \\
\hline 30 & 1 & 2.814475 & -1.394304 & -1.868787 \\
\hline 31 & 6 & 4.827923 & -0.228241 & 1.223635 \\
\hline 32 & 1 & 2.959165 & 0.566978 & 1.947652 \\
\hline 33 & 6 & 5.480023 & -0.923599 & 0.201875 \\
\hline 34 & 1 & 5.233762 & -1.876145 & -1.723070 \\
\hline 35 & 1 & 5.384355 & 0.103613 & 2.097880 \\
\hline 36 & 1 & 6.544904 & -1.134178 & 0.268800 \\
\hline
\end{tabular}

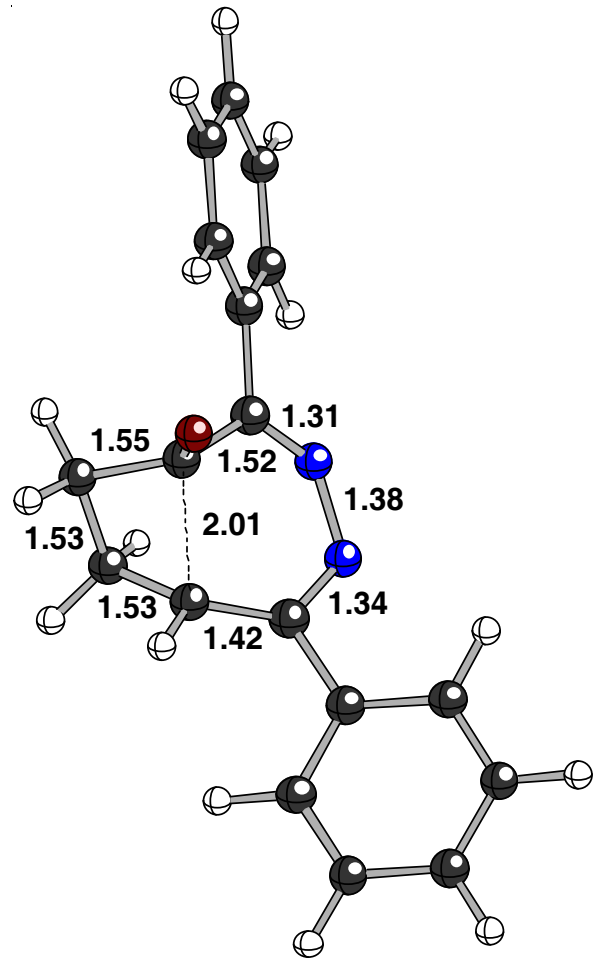




\section{TS4 $\rightarrow 7$ (n=1, Figure 2)}

$\mathrm{HF}=-879.6557715$ hartrees $(-551992.793173965 \mathrm{kcal} / \mathrm{mol})$

Imaginary Frequencies: 1 (-559.3049 1/cm)

Zero-point correction $=0.281578$ (Hartree/Particle)

Temperature 298.150 Kelvin. Pressure 1.00000 Atm.

Sum of electronic and thermal Free Energies = -879.419445 hartrees $\mathbf{( - 5 5 1 8 4 4 . 4 9 5 9 3 1 9 5} \mathbf{~ k c a l} / \mathrm{mol})$

Solvent Single Point: HF = -879.7404514 hartrees $(-552045.9307 \mathrm{kcal} / \mathrm{mol})$

Coordinates (from last standard orientation):

\begin{tabular}{|c|c|c|c|c|}
\hline \multirow{2}{*}{$\begin{array}{l}\text { Center } \\
\text { Number }\end{array}$} & \multirow{2}{*}{$\begin{array}{l}\text { Atomic } \\
\text { Number }\end{array}$} & \multicolumn{3}{|c|}{ Coordinates (Angstroms) } \\
\hline & & $\mathrm{X}$ & $\mathrm{Y}$ & Z \\
\hline \multicolumn{5}{|c|}{ 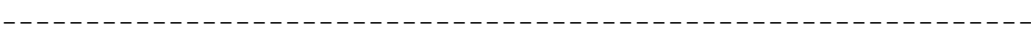 } \\
\hline 1 & 6 & $-\odot .919943$ & 1.474097 & -0.152301 \\
\hline 2 & 6 & -1.350388 & 0.030557 & -0.455709 \\
\hline 3 & 6 & 1.033289 & 1.422237 & -0.735063 \\
\hline 4 & 1 & 0.693949 & 1.821927 & -1.680850 \\
\hline 5 & 6 & 1.475209 & 0.064732 & -0.722313 \\
\hline 6 & 8 & -1.546057 & 2.370910 & -0.787034 \\
\hline 7 & 7 & -0.655118 & $-\odot .898551$ & -1.059018 \\
\hline 8 & 7 & ๑. 725046 & -0.960242 & -1.129513 \\
\hline 9 & 6 & 2.826695 & $-\odot .331501$ & -0.215598 \\
\hline 10 & 6 & 3.066654 & -1.632548 & ๑. 265161 \\
\hline 11 & 6 & 3.903013 & $\odot .573796$ & -0.239129 \\
\hline 12 & 6 & 4.335454 & -2.012187 & ๑. 705842 \\
\hline 13 & 1 & 2.235713 & -2.330121 & $\odot .274420$ \\
\hline 14 & 6 & 5.170559 & ๑. 200061 & ๑. 211165 \\
\hline 15 & 1 & 3.732413 & 1.571145 & -0.634627 \\
\hline 16 & 6 & 5.394240 & -1.096842 & ๑. 686764 \\
\hline 17 & 1 & 4.499155 & -3.023191 & 1.072683 \\
\hline 18 & 1 & 5.987794 & ๑. 917406 & 0.182095 \\
\hline 19 & 1 & 6.381475 & -1.390811 & 1.035690 \\
\hline 20 & 6 & -2.743753 & -0.345872 & -0.103078 \\
\hline 21 & 6 & -3.206784 & -1.668069 & -0.294136 \\
\hline 22 & 6 & -3.645938 & ๑. 587771 & ๑. 450193 \\
\hline 23 & 6 & -4.508274 & -2.032539 & 0.039005 \\
\hline 24 & 1 & -2.513575 & -2.389637 & -0.712139 \\
\hline 25 & 6 & -4.949221 & ๑. 214723 & ๑. 791867 \\
\hline 26 & 1 & -3.328856 & 1.615781 & 0.578536 \\
\hline 27 & 6 & -5.393069 & -1.094349 & 0.590166 \\
\hline 28 & 1 & -4.835693 & -3.057888 & -0.121666 \\
\hline 29 & 1 & -5.622598 & ๑. 958565 & 1.212558 \\
\hline 30 & 1 & -6.406803 & -1.382379 & ๑. 858997 \\
\hline 31 & 6 & 1.137885 & 2.342335 & $\odot .479943$ \\
\hline 32 & 6 & -0.181863 & 1.886492 & 1.152202 \\
\hline 33 & 1 & ๑. 008681 & 1.001781 & 1.776912 \\
\hline 34 & 1 & -0.730689 & 2.639117 & 1.740428 \\
\hline 35 & 1 & 2.024519 & 2.196692 & 1.109723 \\
\hline 36 & 1 & 1. 090881 & 3.398520 & ๑.193332 \\
\hline
\end{tabular}

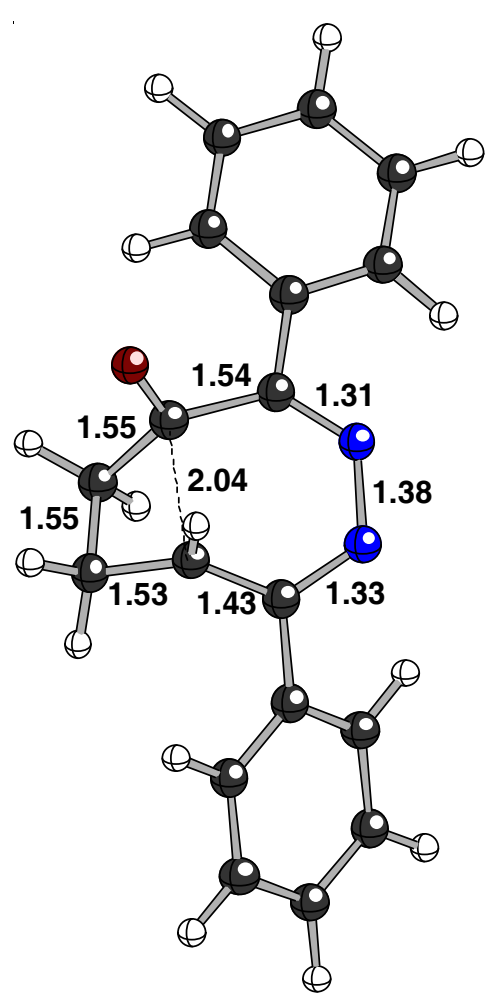




\section{$6(n=1$, Figure 2 and pyridazine figure and scheme in SI)}

$\mathrm{HF}=-879.7130114$ hartrees $(-552028.711783614 \mathrm{kcal} / \mathrm{mol})$

Imaginary Frequencies: none found

Zero-point correction $=0.284251$ (Hartree/Particle)

Temperature 298.150 Kelvin. Pressure 1.00000 Atm.

Sum of electronic and thermal Free Energies = -879.475004 hartrees $(-551879.35976004 \mathrm{kcal} / \mathrm{mol})$

Solvent Single Point: HF $=\mathbf{- 8 7 9 . 7 9 4 8 0 7 9}$ hartrees $(-552080.0399 \mathrm{kcal} / \mathrm{mol})$

Coordinates (from last standard orientation):

\begin{tabular}{|c|c|c|c|c|}
\hline \multirow{2}{*}{$\begin{array}{l}\text { Center } \\
\text { Number }\end{array}$} & \multirow{2}{*}{$\begin{array}{l}\text { Atomic } \\
\text { Number }\end{array}$} & \multicolumn{3}{|c|}{ Coordinates (Angstroms) } \\
\hline & & $\mathrm{x}$ & $\mathrm{Y}$ & Z \\
\hline 1 & 6 & -0.419523 & 2.935685 & 0.617206 \\
\hline 2 & 1 & -0.220899 & 2.614257 & 1.643742 \\
\hline 3 & 1 & -1.032214 & 3.843758 & 0.646210 \\
\hline 4 & 6 & 0.896570 & 3.225877 & $-\odot .102966$ \\
\hline 5 & 1 & 1.463254 & 4. 018914 & 0.415316 \\
\hline 6 & 1 & 0.664567 & 3.624359 & -1.101438 \\
\hline 7 & 6 & -1.243815 & 1.902753 & -0.164454 \\
\hline 8 & 6 & -1.298721 & $\odot .551702$ & 0.342813 \\
\hline 9 & 6 & 1.754824 & 2.007031 & $-\odot .259549$ \\
\hline 10 & 1 & 2.580289 & 2.115625 & -0.962737 \\
\hline 11 & 6 & 1.645914 & 0.808011 & 0.357782 \\
\hline 12 & 8 & -1.937836 & 2.350239 & -1.108295 \\
\hline 13 & 7 & -0.464082 & 0.124371 & 1.352287 \\
\hline 14 & 7 & $\odot .801928$ & $\odot .348746$ & 1.425676 \\
\hline 15 & 6 & 2.663034 & -0.253742 & 0.048766 \\
\hline 16 & 6 & 2.272264 & -1.604560 & 0.017269 \\
\hline 17 & 6 & 4.012244 & 0.044234 & -0.212362 \\
\hline 18 & 6 & 3.184772 & -2.611599 & -0.301819 \\
\hline 19 & 1 & 1.241375 & -1.855156 & 0.242253 \\
\hline 20 & 6 & 4.929209 & -0.962093 & -0.526483 \\
\hline 21 & 1 & 4.351026 & 1.074184 & $-\odot .150296$ \\
\hline 22 & 6 & 4.519604 & -2.297669 & -0.577470 \\
\hline 23 & 1 & 2.851361 & -3.646096 & -0.333036 \\
\hline 24 & 1 & 5.968010 & -0.702302 & $-\odot .717576$ \\
\hline 25 & 1 & 5.231803 & -3.082939 & -0.818720 \\
\hline 26 & 6 & -2.445690 & -0.344241 & ๑.094805 \\
\hline 27 & 6 & -2.537619 & -1.586333 & 0.776340 \\
\hline 28 & 6 & -3.491374 & -0.041858 & -0.813459 \\
\hline 29 & 6 & -3.616750 & -2.447673 & ๑.590381 \\
\hline 30 & 1 & -1.745476 & -1.852399 & 1.467162 \\
\hline 31 & 6 & -4.562765 & -0.917647 & -1.002036 \\
\hline 32 & 1 & -3.440167 & 0.887605 & -1.365119 \\
\hline 33 & 6 & -4.645723 & -2.127146 & -0.304358 \\
\hline 34 & 1 & -3.648875 & -3.385059 & 1.143379 \\
\hline 35 & 1 & -5.344423 & -0.646396 & -1.709843 \\
\hline 36 & 1 & -5.480583 & -2.806480 & -0.461112 \\
\hline
\end{tabular}

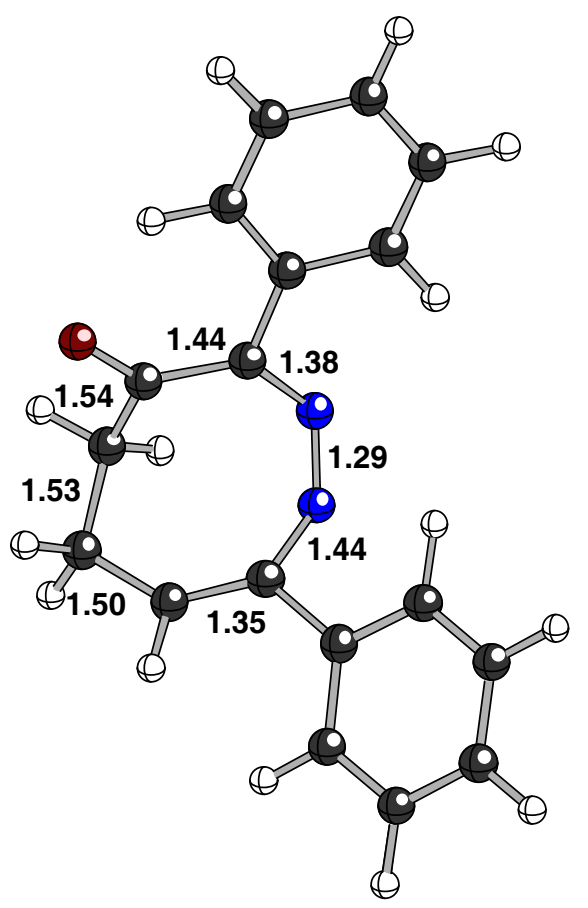




\section{6 ( $n=1$, Figure 2 and pyridazine figure and scheme in SI)}

\section{(solvent optimization, methanol, uaks radii)}

$\mathrm{HF}=-879.796034$ hartrees $(-552080.80929534 \mathrm{kcal} / \mathrm{mol})$

Imaginary Frequencies: none found

Zero-point correction $=0.283873$ (Hartree/Particle)

Temperature 298.150 Kelvin. Pressure 1.00000 Atm.

Sum of electronic and thermal Free Energies = -879.558866 hartrees $(-551931.98400366 \mathrm{kcal} / \mathrm{mol})$

Coordinates (from last standard orientation):

\begin{tabular}{|c|c|c|c|c|}
\hline \multirow{2}{*}{$\begin{array}{l}\text { Center } \\
\text { Number }\end{array}$} & \multirow{2}{*}{$\begin{array}{l}\text { Atomic } \\
\text { Number }\end{array}$} & \multicolumn{3}{|c|}{ Coordinates (Angstroms) } \\
\hline & & $\mathrm{x}$ & $\mathrm{Y}$ & z \\
\hline 1 & 6 & -0.454686 & 2.996261 & $\odot .547075$ \\
\hline 2 & 1 & -0.255391 & 2.731332 & 1.591209 \\
\hline 3 & 1 & -1.058582 & 3.912382 & 0.538979 \\
\hline 4 & 6 & 0.864379 & 3.249185 & $-\odot .186879$ \\
\hline 5 & 1 & 1.445772 & 4.039208 & 0.318712 \\
\hline 6 & 1 & $\odot .646103$ & 3.639584 & -1.192938 \\
\hline 7 & 6 & -1.283112 & 1.928389 & -0.167392 \\
\hline 8 & 6 & -1.310523 & 0.594018 & $\odot .356889$ \\
\hline 9 & 6 & 1.705613 & 2.017724 & -0.331159 \\
\hline 10 & 1 & 2.516422 & 2.101262 & -1.054804 \\
\hline 11 & 6 & 1.619001 & 0.840942 & 0.327156 \\
\hline 12 & 8 & -2.017404 & 2.328621 & -1.118953 \\
\hline 13 & 7 & -0.472682 & $\odot .216332$ & 1.396901 \\
\hline 14 & 7 & 0.792911 & 0.446836 & 1.442864 \\
\hline 15 & 6 & 2.628102 & $-\odot .234961$ & 0.047857 \\
\hline 16 & 6 & 2.229084 & -1.583404 & 0.014667 \\
\hline 17 & 6 & 3.982615 & 0.060248 & -0.192488 \\
\hline 18 & 6 & 3.142934 & -2.598825 & -0.278427 \\
\hline 19 & 1 & 1.192019 & -1.835328 & 0.212228 \\
\hline 20 & 6 & 4.899216 & $-\odot .953919$ & -0.483401 \\
\hline 21 & 1 & 4.325609 & 1.090236 & -0.138175 \\
\hline 22 & 6 & 4.483492 & -2.288715 & -0.530640 \\
\hline 23 & 1 & 2.806713 & -3.632929 & -0.309435 \\
\hline 24 & 1 & 5.942112 & $-\odot .700504$ & $-\odot .661148$ \\
\hline 25 & 1 & 5.197288 & -3.078448 & -0.753244 \\
\hline 26 & 6 & -2.411934 & $-\odot .358807$ & 0.102976 \\
\hline 27 & 6 & -2.301295 & -1.695201 & 0.561631 \\
\hline 28 & 6 & -3.607104 & -0.017785 & -0.576765 \\
\hline 29 & 6 & -3.331875 & -2.620987 & 0.387788 \\
\hline 30 & 1 & -1.390595 & -2.004389 & 1.064003 \\
\hline 31 & 6 & -4.630005 & $-\odot .950567$ & -0.759728 \\
\hline 32 & 1 & -3.716720 & 0.987351 & -0.962145 \\
\hline 33 & 6 & -4.508194 & -2.259364 & -0.277634 \\
\hline 34 & 1 & -3.206183 & -3.635399 & 0.761794 \\
\hline 35 & 1 & -5.534787 & -0.648081 & -1.284026 \\
\hline 36 & 1 & -5.306400 & -2.982816 & -0.426905 \\
\hline
\end{tabular}

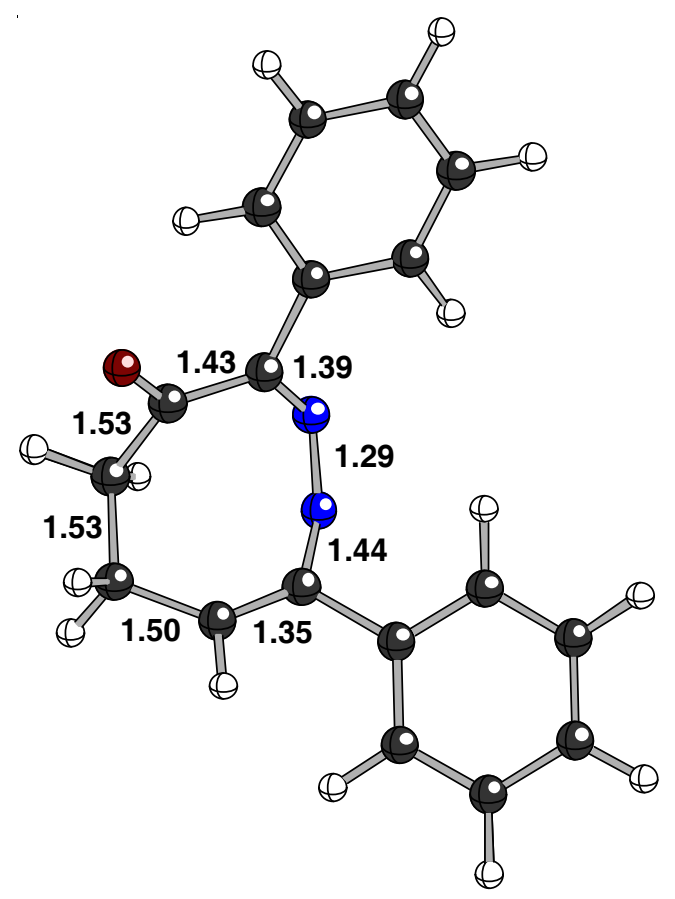




\section{7 (n=1, Figure 2)}

$\mathrm{HF}=-879.6771575$ hartrees $(-552006.213102825 \mathrm{kcal} / \mathrm{mol})$

Imaginary Frequencies: none found

Zero-point correction $=0.283554$ (Hartree/Particle)

Temperature 298.150 Kelvin. Pressure 1.00000 Atm.

Sum of electronic and thermal Free Energies = -879.439440 hartrees $(-551857.0429944 \mathbf{~ k c a l} / \mathrm{mol})$

Solvent Single Point: HF $=\mathbf{- 8 7 9 . 7 5 9 4 7 2 3 ~ h a r t r e e s ~}(-552057.8665 \mathrm{kcal} / \mathrm{mol})$

Coordinates (from last standard orientation):

\begin{tabular}{|c|c|c|c|c|}
\hline \multirow{2}{*}{$\begin{array}{l}\text { Center } \\
\text { Number }\end{array}$} & \multirow{2}{*}{$\begin{array}{l}\text { Atomic } \\
\text { Number }\end{array}$} & \multicolumn{3}{|c|}{ Coordinates (Angstroms) } \\
\hline & & $\mathrm{x}$ & $\mathrm{Y}$ & Z \\
\hline 1 & 6 & -0.956144 & 1.556052 & 0.290628 \\
\hline 2 & 6 & -1.294573 & 0.182188 & -0.202785 \\
\hline 3 & 6 & 1.278161 & 1.373892 & -1.303010 \\
\hline 4 & 1 & $\odot .392708$ & 1.502055 & -1.926669 \\
\hline 5 & 6 & 1.559704 & $\odot .071909$ & -1.010745 \\
\hline 6 & 8 & -1.833462 & 2.436345 & 0.319288 \\
\hline 7 & 7 & -0.618227 & $-\odot .759391$ & -0.904091 \\
\hline 8 & 7 & 0.617984 & -0.936812 & -1.310521 \\
\hline 9 & 6 & 2.786504 & -0.384813 & -0.318460 \\
\hline 10 & 6 & 2.766155 & -1.552168 & 0.469772 \\
\hline 11 & 6 & 4.012354 & 0.296142 & -0.458148 \\
\hline 12 & 6 & 3.918865 & -2.000792 & 1.117755 \\
\hline 13 & 1 & 1.831233 & -2.096123 & 0.555438 \\
\hline 14 & 6 & 5.161718 & -0.145610 & 0.197252 \\
\hline 15 & 1 & 4.053632 & 1.164794 & -1.109142 \\
\hline 16 & 6 & 5.121606 & -1.297769 & ๑. 992342 \\
\hline 17 & 1 & 3.877846 & -2.902550 & 1.724444 \\
\hline 18 & 1 & 6.096016 & 0.397749 & $\odot .073969$ \\
\hline 19 & 1 & 6. 019169 & -1.648591 & 1.495982 \\
\hline 20 & 6 & -2.726244 & -0.226817 & -0.017682 \\
\hline 21 & 6 & -3.322015 & -1.172808 & $-\odot .881895$ \\
\hline 22 & 6 & -3.527438 & 0.242338 & 1.047354 \\
\hline 23 & 6 & -4.629222 & -1.622818 & -0.693323 \\
\hline 24 & 1 & -2.723046 & -1.557998 & -1.699149 \\
\hline 25 & 6 & -4.833870 & $-\odot .209257$ & 1.238667 \\
\hline 26 & 1 & -3.124486 & 0.978668 & 1.732911 \\
\hline 27 & 6 & -5.403697 & -1.147291 & ๑.370491 \\
\hline 28 & 1 & -5.047336 & -2.348876 & -1.388533 \\
\hline 29 & 1 & -5.411141 & 0.176977 & 2.076836 \\
\hline 30 & 1 & -6.423748 & -1.494786 & 0.517616 \\
\hline 31 & 6 & 1.414952 & 2.455108 & -0.270030 \\
\hline 32 & 6 & $\odot .419585$ & 1.971739 & ๑.841598 \\
\hline 33 & 1 & ๑.896856 & 1.121730 & 1.341367 \\
\hline 34 & 1 & $\odot .227243$ & 2.762570 & 1.578366 \\
\hline 35 & 1 & 2.412413 & 2.555310 & 0.170618 \\
\hline 36 & 1 & 1.113640 & 3.436372 & -0.654765 \\
\hline
\end{tabular}

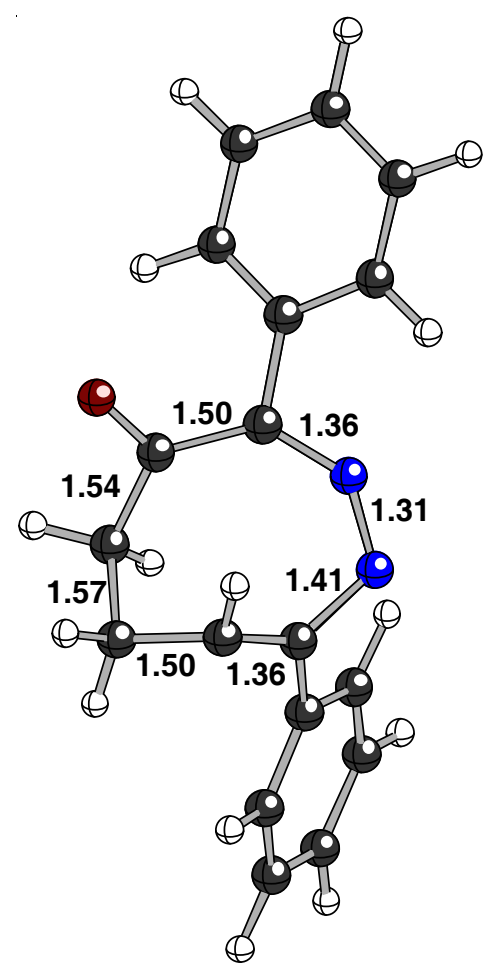




\section{Reactant, $X=\mathbf{O}$ (Table 1)}

$\mathrm{HF}=-915.5917094$ hartrees $(-574542.953565594 \mathrm{kcal} / \mathrm{mol})$

Imaginary Frequencies: none found

Zero-point correction $=0.260198$ (Hartree/Particle)

Temperature 298.150 Kelvin. Pressure 1.00000 Atm.

Sum of electronic and thermal Free Energies $=\mathbf{- 9 1 5 . 3 7 7 0 4 9}$ hartrees $(-574408.25201799 \mathrm{kcal} / \mathrm{mol})$

Solvent Single Point: HF = $\mathbf{- 9 1 5 . 6 9 2 5 4 7 9}$ hartrees $(-574606.2307 \mathrm{kcal} / \mathrm{mol})$

Coordinates (from last standard orientation):

\begin{tabular}{|c|c|c|c|c|}
\hline \multirow{2}{*}{$\begin{array}{l}\text { Center } \\
\text { Number }\end{array}$} & \multirow{2}{*}{$\begin{array}{l}\text { Atomic } \\
\text { Number }\end{array}$} & \multicolumn{3}{|c|}{ Coordinates (Angstroms) } \\
\hline & & $\mathrm{x}$ & $\mathrm{Y}$ & Z \\
\hline 1 & 6 & 0.800195 & 2.084417 & -0.885828 \\
\hline 2 & 1 & 1.510078 & 1.832538 & -1.684654 \\
\hline 3 & 1 & 0.882156 & 3.147028 & $-\odot .616396$ \\
\hline 4 & 6 & $\odot .717617$ & 1.182853 & 0.411051 \\
\hline 5 & 6 & 1.298429 & -0.215099 & 0.155711 \\
\hline 6 & 6 & -0.812151 & 1.157721 & 0.059293 \\
\hline 7 & 1 & -1.358136 & 1.868097 & 0.694550 \\
\hline 8 & 6 & -1.464288 & -0.188706 & 0.037830 \\
\hline 9 & 8 & 1.116582 & 1.620806 & 1.598451 \\
\hline 10 & 7 & 0.607665 & -1.318214 & 0.063250 \\
\hline 11 & 7 & -0.790391 & -1.297497 & 0.042054 \\
\hline 12 & 8 & -0.564289 & 1.730344 & -1.260643 \\
\hline 13 & 6 & 2.777130 & -0.383321 & 0.089257 \\
\hline 14 & 6 & 3.317842 & -1.494517 & -0.588050 \\
\hline 15 & 6 & 3.665182 & 0.563957 & 0.635944 \\
\hline 16 & 6 & 4.698032 & -1.654025 & -0.717555 \\
\hline 17 & 1 & 2.632599 & -2.223622 & -1.006718 \\
\hline 18 & 6 & 5.045637 & 0.402452 & ๑.497741 \\
\hline 19 & 1 & 3.231391 & 1.392769 & 1.188584 \\
\hline 20 & 6 & 5.572125 & -0.704939 & -0.175934 \\
\hline 21 & 1 & 5.092360 & -2.519033 & -1.246571 \\
\hline 22 & 1 & 5.715119 & 1.141833 & ๑.931996 \\
\hline 23 & 1 & 6.648280 & -0.828858 & -0.275618 \\
\hline 24 & 6 & -2.945175 & -0.301499 & ๑. 045990 \\
\hline 25 & 6 & -3.747783 & 0.799761 & -0.303416 \\
\hline 26 & 6 & -3.583315 & -1.511371 & 0.383867 \\
\hline 27 & 6 & -5.141601 & 0.696795 & -0.312682 \\
\hline 28 & 1 & -3.275551 & 1.734169 & -0.590331 \\
\hline 29 & 6 & -4.972722 & -1.611593 & ๑.376445 \\
\hline 30 & 1 & -2.961129 & -2.359618 & ๑.648090 \\
\hline 31 & 6 & -5.762233 & -0.507044 & ๑.029566 \\
\hline 32 & 1 & -5.741664 & 1.559517 & $-\odot .591815$ \\
\hline 33 & 1 & -5.445691 & $-2.55289 \odot$ & 0.646947 \\
\hline 34 & 1 & -6.846607 & -0.586201 & ๑. 026971 \\
\hline
\end{tabular}

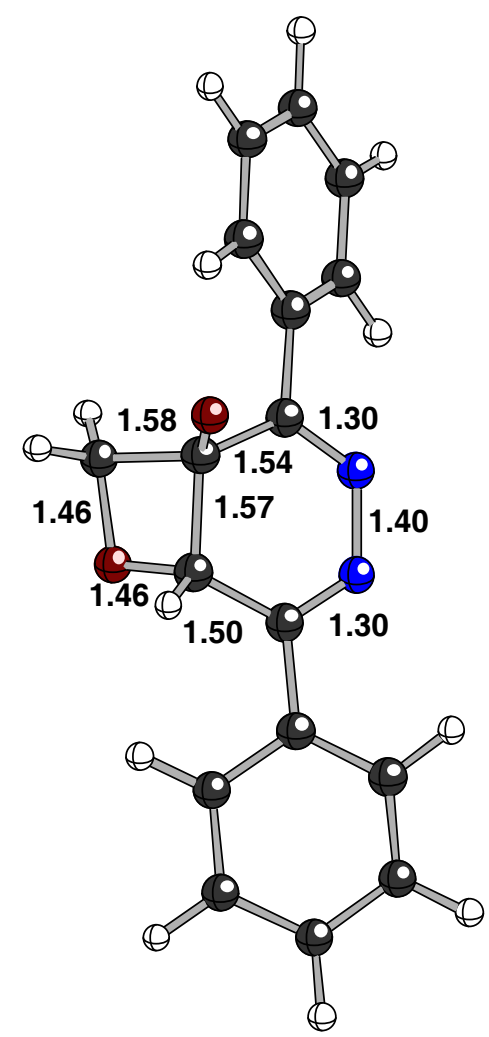




\section{Electrocyclic TS, $\mathrm{X}=\mathrm{O}$ (Table 1)}

$\mathrm{HF}=-915.5829487$ hartrees $(-574537.456138737 \mathrm{kcal} / \mathrm{mol})$

Imaginary Frequencies: $1(-319.7877$ 1/cm)

Zero-point correction $=0.259010$ (Hartree/Particle)

Temperature 298.150 Kelvin. Pressure 1.00000 Atm.

Sum of electronic and thermal Free Energies $=\mathbf{- 9 1 5 . 3 6 8 5 0 3}$ hartrees $(-574402.88931753 \mathrm{kcal} / \mathrm{mol})$

Solvent Single Point: HF = -915.6761506 hartrees $(-574595.9413 \mathrm{kcal} / \mathrm{mol})$

Coordinates (from last standard orientation):

\begin{tabular}{|c|c|c|c|c|}
\hline \multirow{2}{*}{$\begin{array}{l}\text { Center } \\
\text { Number }\end{array}$} & \multirow{2}{*}{$\begin{array}{l}\text { Atomic } \\
\text { Number }\end{array}$} & \multicolumn{3}{|c|}{ Coordinates (Angstroms) } \\
\hline & & $\mathrm{x}$ & $\mathrm{Y}$ & Z \\
\hline 1 & 6 & 0.843170 & 2.392029 & -0.628184 \\
\hline 2 & 1 & 1.766799 & 2.441292 & -1.222362 \\
\hline 3 & 1 & 0.640998 & 3.373642 & -0.170407 \\
\hline 4 & 6 & 0.772580 & 1.297523 & 0.468850 \\
\hline 5 & 6 & 1.250921 & -0.025144 & -0.127908 \\
\hline 6 & 6 & -1.009075 & 1.374044 & -0.346684 \\
\hline 7 & 1 & -1.618854 & 2.111908 & 0.181194 \\
\hline 8 & 6 & -1.522124 & 0.054245 & -0.500447 \\
\hline 9 & 8 & 0.991174 & 1.564591 & 1.687464 \\
\hline 10 & 7 & 0.578790 & -0.924621 & -0.809414 \\
\hline 11 & 7 & -0.782406 & -0.977684 & -0.890274 \\
\hline 12 & 8 & -0.267173 & 1.947753 & -1.417008 \\
\hline 13 & 6 & 2.694807 & -0.362496 & 0.056568 \\
\hline 14 & 6 & 3.325735 & -1.228058 & -0.865672 \\
\hline 15 & 6 & 3.484249 & 0.177467 & 1.092755 \\
\hline 16 & 6 & 4.680390 & -1.534460 & -0.759492 \\
\hline 17 & 1 & 2.722300 & -1.650271 & -1.661359 \\
\hline 18 & 6 & 4.844807 & -0.131601 & 1.192184 \\
\hline 19 & 1 & 3.006753 & 0.825188 & 1.818925 \\
\hline 20 & 6 & 5.454671 & -0.988323 & 0.272784 \\
\hline 21 & 1 & 5.137340 & -2.200705 & -1.488518 \\
\hline 22 & 1 & 5.427297 & 0.296250 & 2.005493 \\
\hline 23 & 1 & 6.511813 & -1.229834 & 0.357507 \\
\hline 24 & 6 & -2.923714 & -0.267298 & -0.113396 \\
\hline 25 & 6 & -3.941522 & 0.703324 & -0.146736 \\
\hline 26 & 6 & -3.267402 & -1.573358 & ๑.286656 \\
\hline 27 & 6 & -5.252886 & 0.385336 & 0.215810 \\
\hline 28 & 1 & -3.705191 & 1.709571 & -0.478407 \\
\hline 29 & 6 & -4.577936 & -1.894300 & 0.637722 \\
\hline 30 & 1 & -2.480921 & -2.319987 & 0.309135 \\
\hline 31 & 6 & -5.578916 & -0.915459 & 0.609605 \\
\hline 32 & 1 & -6.022285 & 1.153108 & 0.180509 \\
\hline 33 & 1 & -4.819938 & -2.909099 & 0.945183 \\
\hline 34 & 1 & -6.599343 & -1.164516 & 0.890777 \\
\hline
\end{tabular}

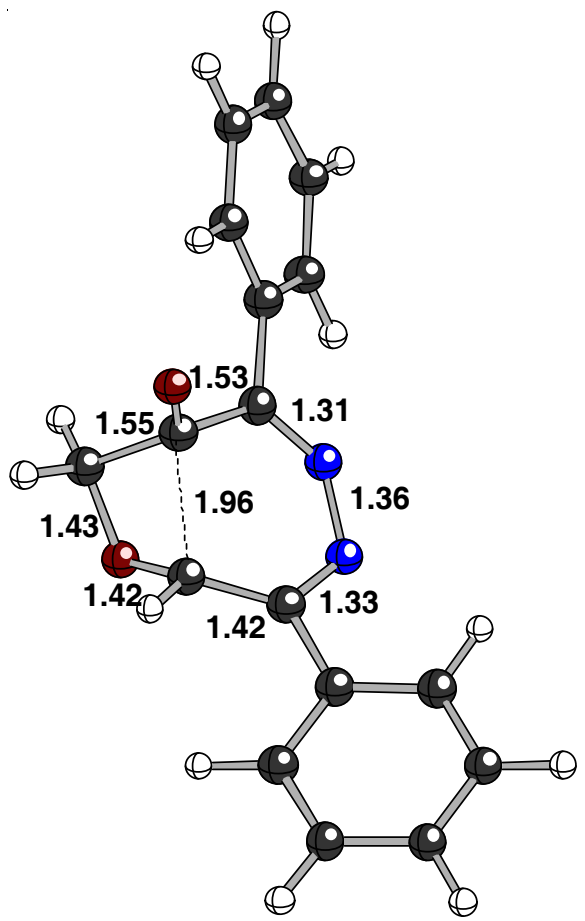




\section{Fragmentation TS, $X=O$ (Table 1)}

$\mathrm{HF}=-915.5467404$ hartrees $(-574514.735068404 \mathrm{kcal} / \mathrm{mol})$

Imaginary Frequencies: $1(-477.7999$ 1/cm)

Zero-point correction $=0.257439$ (Hartree/Particle)

Temperature 298.150 Kelvin. Pressure 1.00000 Atm.

Sum of electronic and thermal Free Energies $=\mathbf{- 9 1 5 . 3 3 4 6 4 0 ~ h a r t r e e s ~}(-574381.6399464 \mathrm{kcal} / \mathrm{mol})$

Solvent Single Point: HF = $\mathbf{- 9 1 5 . 6 3 2 6 2 0 8 ~ h a r t r e e s ~}(-574568.6259 \mathrm{kcal} / \mathrm{mol})$

Coordinates (from last standard orientation):

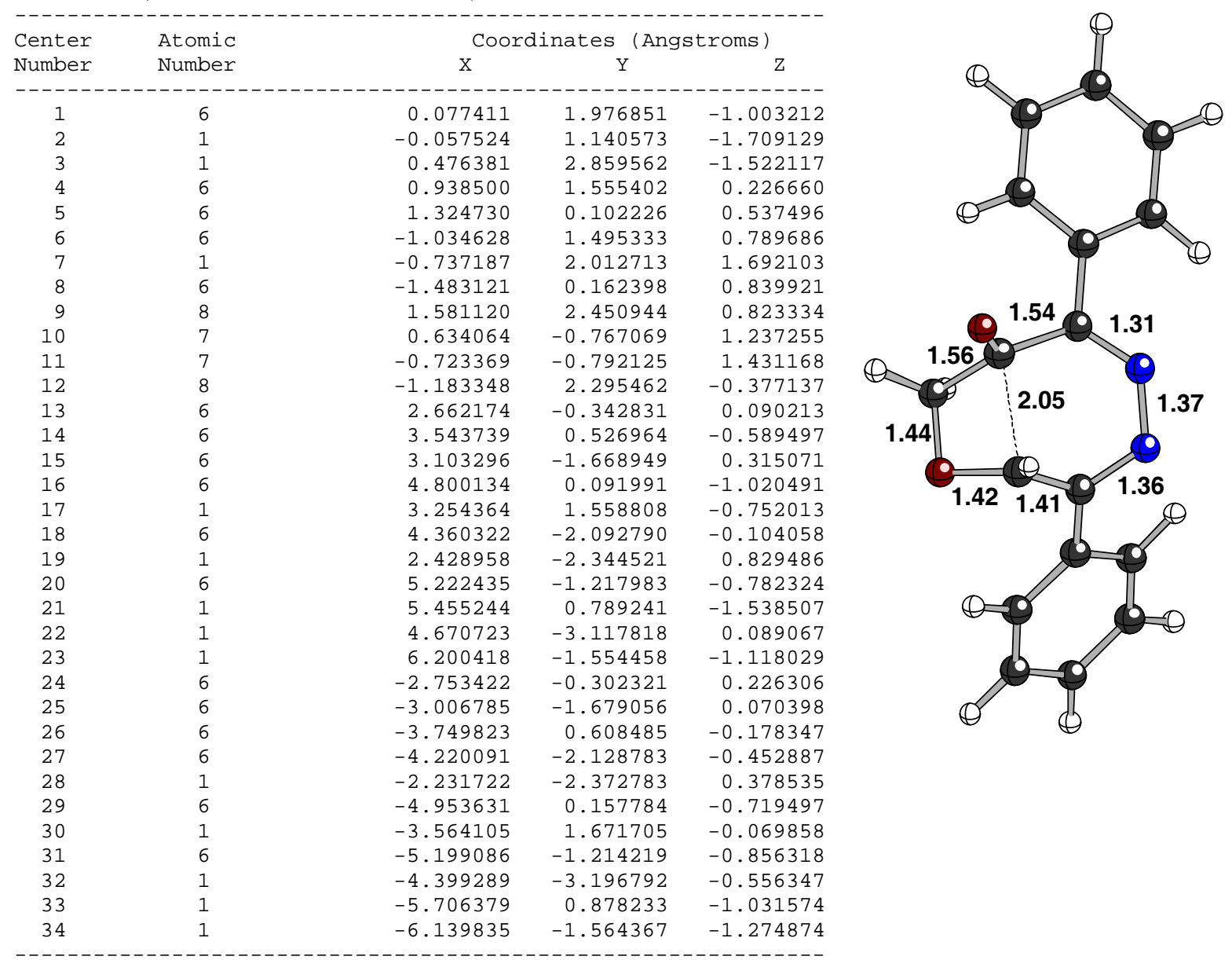




\section{Electrocyclic product, $X=\mathbf{O}$ (Table 1)}

$\mathrm{HF}=-915.6035481$ hartrees $(-574550.382468231 \mathrm{kcal} / \mathrm{mol})$

Imaginary Frequencies: none found

Zero-point correction $=0.259668$ (Hartree/Particle)

Temperature 298.150 Kelvin. Pressure 1.00000 Atm.

Sum of electronic and thermal Free Energies $=\mathbf{- 9 1 5 . 3 9 0 7 6 1}$ hartrees $(-574416.85643511 \mathrm{kcal} / \mathrm{mol})$

Solvent Single Point: HF $=\mathbf{- 9 1 5 . 6 8 4 3 8 0 4}$ hartrees $(-574601.1055 \mathrm{kcal} / \mathrm{mol})$

Coordinates (from last standard orientation):

\begin{tabular}{|c|c|c|c|c|}
\hline \multirow{2}{*}{$\begin{array}{l}\text { Center } \\
\text { Number }\end{array}$} & \multirow{2}{*}{$\begin{array}{l}\text { Atomic } \\
\text { Number }\end{array}$} & \multicolumn{3}{|c|}{ Coordinates (Angstroms) } \\
\hline & & $\mathrm{x}$ & $\mathrm{Y}$ & Z \\
\hline 1 & 6 & ๑.399105 & 2.735116 & -0.442113 \\
\hline 2 & 1 & 1.205911 & 3.250278 & -0.979373 \\
\hline 3 & 1 & -0.039570 & 3.442988 & 0.272983 \\
\hline 4 & 6 & 1.081822 & 1.621297 & 0.377900 \\
\hline 5 & 6 & 1.336653 & 0.294711 & -0.175083 \\
\hline 6 & 6 & -1.643398 & 1.736825 & -0.859345 \\
\hline 7 & 1 & -2.508292 & 2.374788 & -0.674207 \\
\hline 8 & 6 & -1.660800 & $\odot .401018$ & $-\odot .660243$ \\
\hline 9 & 8 & 1.624855 & 2.069252 & 1.409697 \\
\hline 10 & 7 & 0.559013 & -0.462556 & -0.998492 \\
\hline 11 & 7 & -0.711548 & -0.504337 & -1.176842 \\
\hline 12 & 8 & -0.560165 & 2.368408 & -1.426156 \\
\hline 13 & 6 & 2.668723 & -0.343435 & 0.059081 \\
\hline 14 & 6 & 3.050145 & -1.477513 & -0.706115 \\
\hline 15 & 6 & 3.620476 & 0.120661 & 1.000255 \\
\hline 16 & 6 & 4.295052 & -2.082978 & -0.558655 \\
\hline 17 & 1 & 2.338899 & -1.866316 & -1.424359 \\
\hline 18 & 6 & 4.867953 & -0.495322 & 1.144098 \\
\hline 19 & 1 & 3.367091 & 0.964535 & 1.626323 \\
\hline 20 & 6 & 5.226460 & -1.601226 & 0.370859 \\
\hline 21 & 1 & 4.539257 & -2.945689 & -1.176511 \\
\hline 22 & 1 & 5.563433 & -0.100871 & 1.883187 \\
\hline 23 & 1 & 6.195584 & -2.079959 & 0.491918 \\
\hline 24 & 6 & -2.877821 & -0.250787 & -0.105355 \\
\hline 25 & 6 & -3.279789 & -1.520149 & -0.563319 \\
\hline 26 & 6 & -3.649649 & 0.366533 & 0.896238 \\
\hline 27 & 6 & -4.426183 & -2.132863 & $-\odot .056056$ \\
\hline 28 & 1 & -2.670103 & -2.006458 & -1.316541 \\
\hline 29 & 6 & -4.799777 & -0.246362 & 1.399717 \\
\hline 30 & 1 & -3.328112 & 1.320587 & 1.302934 \\
\hline 31 & 6 & -5.197794 & -1.499316 & 0.924695 \\
\hline 32 & 1 & -4.718864 & -3.112219 & -0.427773 \\
\hline 33 & 1 & -5.374253 & $\odot .249007$ & 2.178997 \\
\hline 34 & 1 & -6.087823 & -1.980954 & 1.322068 \\
\hline
\end{tabular}

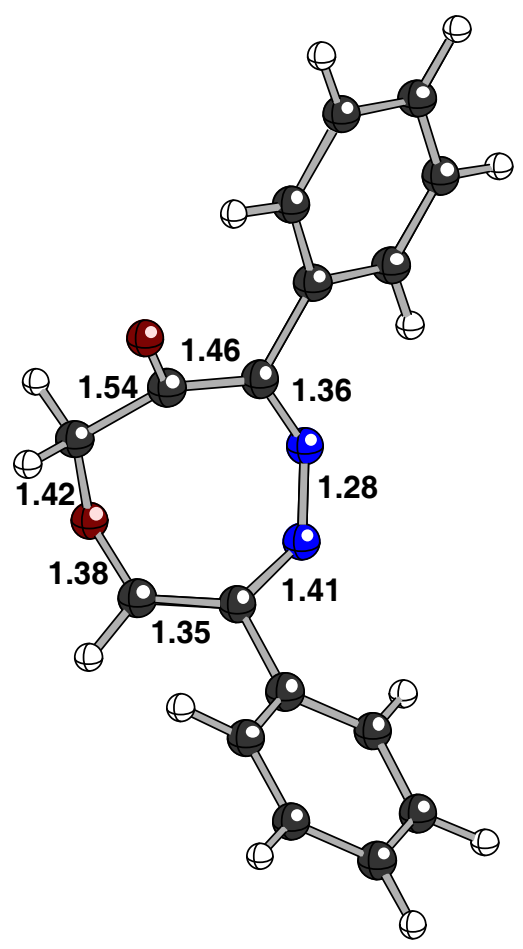




\section{Fragmentation product, $\mathrm{X}=\mathbf{O}$ (Table 1)}

$\mathrm{HF}=-915.5698686$ hartrees $(-574529.248245186 \mathrm{kcal} / \mathrm{mol})$

Imaginary Frequencies: none found

Zero-point correction $=0.259537$ (Hartree/Particle)

Temperature 298.150 Kelvin. Pressure 1.00000 Atm.

Sum of electronic and thermal Free Energies $=\mathbf{- 9 1 5 . 3 5 6 1 8 6}$ hartrees $(-574395.16027686 \mathrm{kcal} / \mathrm{mol})$

Solvent Single Point: HF = $\mathbf{- 9 1 5 . 6 5 2 2 4 2 4}$ hartrees $(-574580.9386 \mathrm{kcal} / \mathrm{mol})$

Coordinates (from last standard orientation):

\begin{tabular}{|c|c|c|c|c|}
\hline \multirow{2}{*}{$\begin{array}{l}\text { Center } \\
\text { Number }\end{array}$} & \multirow{2}{*}{$\begin{array}{l}\text { Atomic } \\
\text { Number }\end{array}$} & \multicolumn{3}{|c|}{ Coordinates (Angstroms) } \\
\hline & & $\mathrm{X}$ & $\mathrm{Y}$ & Z \\
\hline \multicolumn{5}{|c|}{ 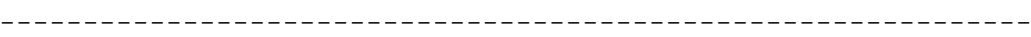 } \\
\hline 1 & 6 & $-\odot .728605$ & 2.042062 & -0.502181 \\
\hline 2 & 1 & -1.579804 & 1.674528 & -1.087363 \\
\hline 3 & 1 & -0.374497 & 2.965805 & -0.967527 \\
\hline 4 & 6 & ๑. 448138 & 1.022851 & -0.671492 \\
\hline 5 & 6 & 1.102766 & ๑. . 091662 & ๑. 284769 \\
\hline 6 & 6 & -1.076439 & 1.335579 & 1.656125 \\
\hline 7 & 1 & -0.396598 & 1.483194 & 2.494287 \\
\hline 8 & 6 & -1.445626 & 0.078543 & 1.306619 \\
\hline 9 & 8 & $\odot .880454$ & 1.112747 & -1.837011 \\
\hline 10 & 7 & $\odot .648275$ & -0.758992 & 1.260050 \\
\hline 11 & 7 & -0.536470 & -0.919853 & 1.775759 \\
\hline 12 & 8 & -1.166107 & 2.418212 & 0.825648 \\
\hline 13 & 6 & 2.550576 & -0.176765 & -0.001004 \\
\hline 14 & 6 & 3.135369 & -1.423539 & ๑. 311007 \\
\hline 15 & 6 & 3.416976 & ๑.812702 & -0.516257 \\
\hline 16 & 6 & 4.497223 & -1.666036 & 0.121022 \\
\hline 17 & 1 & 2.496577 & -2.196713 & 0.721933 \\
\hline 18 & 6 & 4.777525 & 0.572670 & -0.706053 \\
\hline 19 & 1 & 3.015301 & 1.784530 & -0.780555 \\
\hline 20 & 6 & 5.336580 & -0.671451 & -0.390649 \\
\hline 21 & 1 & 4.903066 & -2.644805 & ๑. 370890 \\
\hline 22 & 1 & 5.407002 & 1.367140 & -1.102651 \\
\hline 23 & 1 & 6.397026 & -0.859532 & -0.541942 \\
\hline 24 & 6 & -2.540810 & -0.328002 & 0.417386 \\
\hline 25 & 6 & -3.641180 & $\odot .516194$ & 0.149146 \\
\hline 26 & 6 & -2.552265 & -1.622765 & -0.141695 \\
\hline 27 & 6 & -4.697121 & ๑. 087601 & -0.653178 \\
\hline 28 & 1 & -3.661490 & 1.510404 & 0.585712 \\
\hline 29 & 6 & -3.614195 & -2.050190 & -0.939784 \\
\hline 30 & 1 & -1.714133 & -2.279314 & ๑. . 066241 \\
\hline 31 & 6 & -4.693087 & -1.200169 & -1.204010 \\
\hline 32 & 1 & -5.531645 & ๑.758446 & -0.844899 \\
\hline 33 & 1 & -3.595379 & -3.051560 & -1.363582 \\
\hline 34 & 1 & -5.517789 & -1.533556 & -1.828927 \\
\hline
\end{tabular}

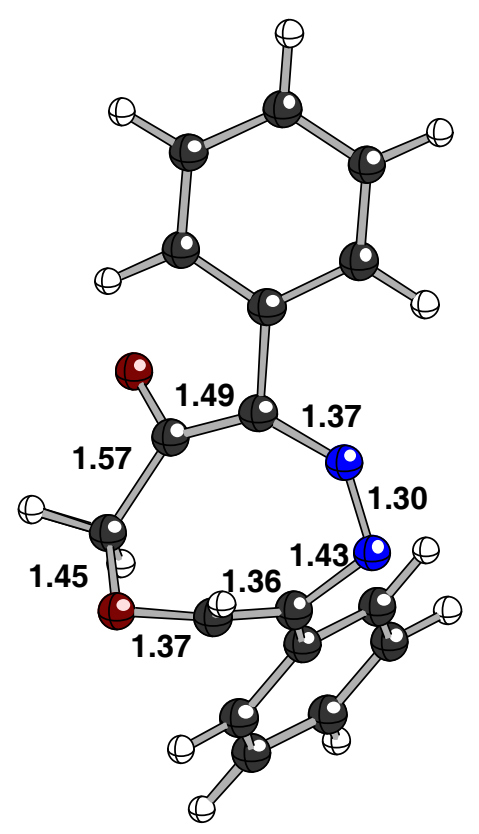




\section{Reactant, $X=S$ (Table 1)}

$\mathrm{HF}=-1238.5832135$ hartrees $(-777223.352303385 \mathrm{kcal} / \mathrm{mol})$

Imaginary Frequencies: none found

Zero-point correction $=0.257754$ (Hartree/Particle)

Temperature 298.150 Kelvin. Pressure 1.00000 Atm.

Sum of electronic and thermal Free Energies = -1238.371576 hartrees $(-777090.54765576 \mathrm{kcal} / \mathrm{mol})$

Solvent Single Point: HF = -1238.678415 hartrees $(-777283.0919$ kcal $/ \mathrm{mol})$

Coordinates (from last standard orientation):

\begin{tabular}{|c|c|c|c|c|}
\hline \multirow{2}{*}{$\begin{array}{l}\text { Center } \\
\text { Number }\end{array}$} & \multirow{2}{*}{$\begin{array}{l}\text { Atomic } \\
\text { Number }\end{array}$} & \multicolumn{3}{|c|}{ Coordinates (Angstroms) } \\
\hline & & $\mathrm{X}$ & $\mathrm{Y}$ & Z \\
\hline \multicolumn{5}{|c|}{ 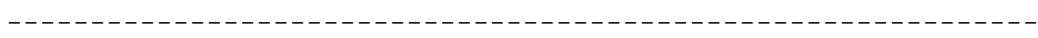 } \\
\hline 1 & 6 & 1.124295 & 2.202033 & -0.267483 \\
\hline 2 & 1 & 2.059919 & 2.119933 & -0.827254 \\
\hline 3 & 1 & 1.100962 & 3.131339 & 0.309214 \\
\hline 4 & 6 & 0.711033 & ๑. 989757 & ๑. 658391 \\
\hline 5 & 6 & 1. 295226 & -0.338272 & $\odot .137787$ \\
\hline 6 & 6 & -0.807075 & 1.025536 & ๑. 237249 \\
\hline 7 & 1 & -1.353995 & 1.663002 & 0.937243 \\
\hline 8 & 6 & -1.455207 & -0.298672 & ๑. . 049253 \\
\hline 9 & 8 & ๑. 980624 & 1.062418 & 1.954554 \\
\hline 10 & 7 & 0.608417 & -1.413122 & -0.135737 \\
\hline 11 & 7 & -0.784480 & -1.396081 & -0.137142 \\
\hline 12 & 16 & -0.405152 & 2.022898 & -1.309029 \\
\hline 13 & 6 & 2.773786 & -0.501601 & ๑. . 077247 \\
\hline 14 & 6 & 3.646309 & ๑. 243843 & ๑. 893808 \\
\hline 15 & 6 & 3.330676 & -1.403465 & -0.849890 \\
\hline 16 & 6 & 5.029600 & ๑. 093440 & 0.774464 \\
\hline 17 & 1 & 3.203096 & ๑. 905719 & 1.631548 \\
\hline 18 & 6 & 4.713804 & -1.552124 & -0.963625 \\
\hline 19 & 1 & 2.656670 & -1.981212 & -1.473189 \\
\hline 20 & 6 & 5.572937 & -0.802711 & -0.152670 \\
\hline 21 & 1 & 5.687313 & ๑. 672202 & 1.419282 \\
\hline 22 & 1 & 5.122008 & -2.252317 & -1.689221 \\
\hline 23 & 1 & 6.651002 & -0.918140 & -0.239997 \\
\hline 24 & 6 & -2.937019 & $-\odot .420817$ & ๑. 073981 \\
\hline 25 & 6 & -3.554523 & -1.682547 & 0.190356 \\
\hline 26 & 6 & -3.762755 & ๑. 713377 & -0.029512 \\
\hline 27 & 6 & -4.942049 & -1.801097 & 0.204893 \\
\hline 28 & 1 & -2.916125 & -2.555683 & $\odot .268192$ \\
\hline 29 & 6 & -5.155402 & ๑. 592597 & -0.018007 \\
\hline 30 & 1 & -3.315685 & 1.696659 & -0.136604 \\
\hline 31 & 6 & -5.753818 & -0.663466 & 0.101540 \\
\hline 32 & 1 & -5.396113 & -2.784581 & 0.302087 \\
\hline 33 & 1 & -5.771212 & 1.484461 & -0.104981 \\
\hline 34 & 1 & -6.836874 & -0.757529 & 0.115172 \\
\hline
\end{tabular}

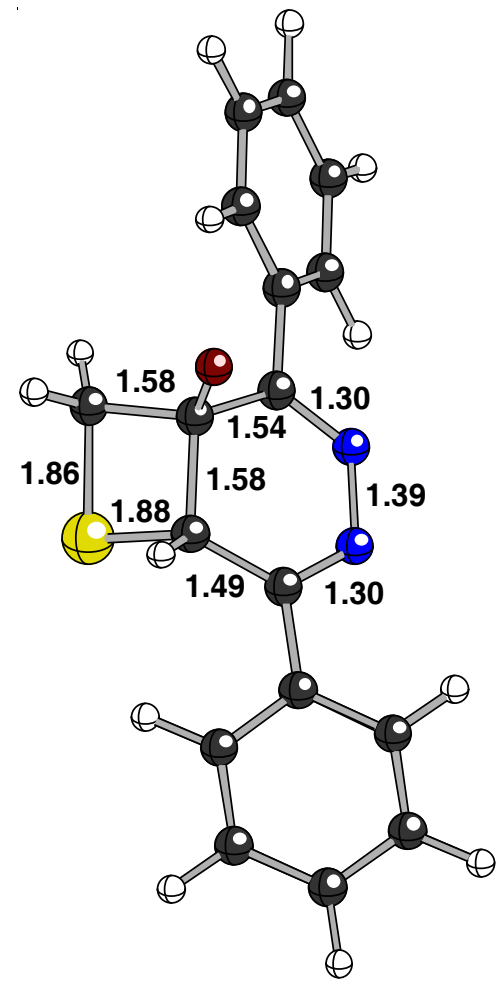




\section{Electrocyclic TS, $X=S$ (Table 1)}

$\mathrm{HF}=-1238.5676217$ hartrees $(-777213.568292967 \mathrm{kcal} / \mathrm{mol})$

Imaginary Frequencies: 1 (-310.4953 1/cm)

Zero-point correction $=0.256011$ (Hartree/Particle)

Temperature 298.150 Kelvin. Pressure 1.00000 Atm.

Sum of electronic and thermal Free Energies = -1238.357251 hartrees $(-777081.55857501 \mathrm{kcal} / \mathrm{mol})$

Solvent Single Point: HF $=\mathbf{- 1 2 3 8 . 6 5 1 3 6 3 ~ h a r t r e e s ~}(-777266.1167 \mathrm{kcal} / \mathrm{mol})$

Coordinates (from last standard orientation):

\begin{tabular}{|c|c|c|c|c|}
\hline \multirow{2}{*}{$\begin{array}{l}\text { Center } \\
\text { Number }\end{array}$} & \multirow{2}{*}{$\begin{array}{l}\text { Atomic } \\
\text { Number }\end{array}$} & \multicolumn{3}{|c|}{ Coordinates (Angstroms) } \\
\hline & & $\mathrm{X}$ & $\mathrm{Y}$ & Z \\
\hline \multicolumn{5}{|c|}{ 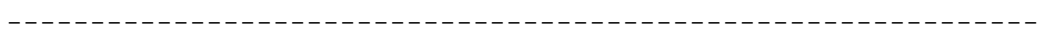 } \\
\hline 1 & 6 & 1.149317 & 2.351792 & 0.208882 \\
\hline 2 & 1 & 2.201127 & 2.440919 & -0.093126 \\
\hline 3 & 1 & 0.897013 & 3.154914 & 0.907475 \\
\hline 4 & 6 & ๑. 807055 & ๑. 989247 & 0.857104 \\
\hline 5 & 6 & 1. 246689 & -0.178474 & -0.032675 \\
\hline 6 & 6 & -1.064199 & 1.304463 & -0.063007 \\
\hline 7 & 1 & -1.705292 & 1.913404 & 0.571928 \\
\hline 8 & 6 & -1.544485 & 0.006003 & -0.394242 \\
\hline 9 & 8 & ๑. 851948 & ๑.853947 & 2.102872 \\
\hline 10 & 7 & $\odot .549237$ & -0.961031 & -0.815408 \\
\hline 11 & 7 & -0.813272 & -0.989742 & -0.880827 \\
\hline 12 & 16 & -0.055197 & 2.304626 & -1.176882 \\
\hline 13 & 6 & 2.698691 & -0.518041 & ๑. . 038847 \\
\hline 14 & 6 & 3.307961 & -1.186105 & -1.046544 \\
\hline 15 & 6 & 3.517033 & -0.163607 & 1.131051 \\
\hline 16 & 6 & 4.668258 & -1.485992 & -1.038557 \\
\hline 17 & 1 & 2.682553 & -1.462523 & -1.888006 \\
\hline 18 & 6 & 4.882729 & -0.464084 & 1.132753 \\
\hline 19 & 1 & 3.065352 & ๑. 323969 & 1.987636 \\
\hline 20 & 6 & 5.470504 & -1.127046 & ๑. 052748 \\
\hline 21 & 1 & 5.108202 & -1.999213 & -1.891194 \\
\hline 22 & 1 & 5.486369 & -0.185302 & 1.993862 \\
\hline 23 & 1 & 6.532226 & -1.362352 & 0.059162 \\
\hline 24 & 6 & -2.967111 & $-\odot .356613$ & -0.105479 \\
\hline 25 & 6 & -3.986235 & ๑. 611549 & -0.087346 \\
\hline 26 & 6 & -3.321072 & -1.695258 & 0.148029 \\
\hline 27 & 6 & -5.311513 & ๑. 258742 & 0.181622 \\
\hline 28 & 1 & -3.738017 & 1.645810 & -0.305251 \\
\hline 29 & 6 & -4.643730 & -2.049949 & 0.411616 \\
\hline 30 & 1 & -2.532987 & -2.440121 & 0.128153 \\
\hline 31 & 6 & -5.647646 & -1.074139 & 0.433713 \\
\hline 32 & 1 & -6.082639 & 1.025662 & ๑. 186142 \\
\hline 33 & 1 & -4.893381 & -3.090011 & 0.608883 \\
\hline 34 & 1 & -6.678200 & -1.350275 & 0.644028 \\
\hline
\end{tabular}

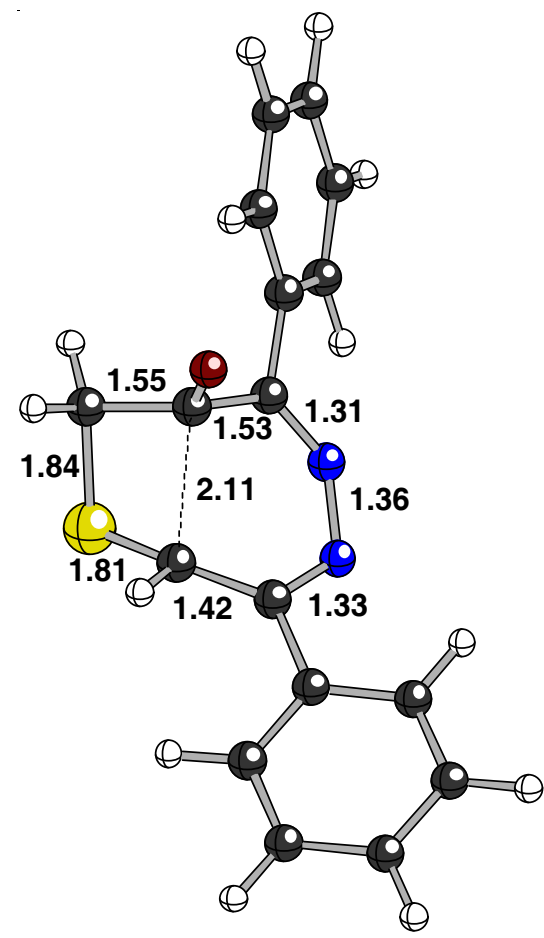




\section{Fragmentation TS, $X=S$ (Table 1)}

$\mathrm{HF}=-1238.5376677$ hartrees $(-777194.771858427 \mathrm{kcal} / \mathrm{mol})$

Imaginary Frequencies: 1 (-465.0351 1/cm)

Zero-point correction $=0.254782$ (Hartree/Particle)

Temperature 298.150 Kelvin. Pressure 1.00000 Atm.

Sum of electronic and thermal Free Energies = -1238.329054 hartrees $(-777063.86467554 \mathrm{kcal} / \mathrm{mol})$

Solvent Single Point: HF = -1238.620849 hartrees $(-777246.9691 \mathrm{kcal} / \mathrm{mol})$

Coordinates (from last standard orientation):

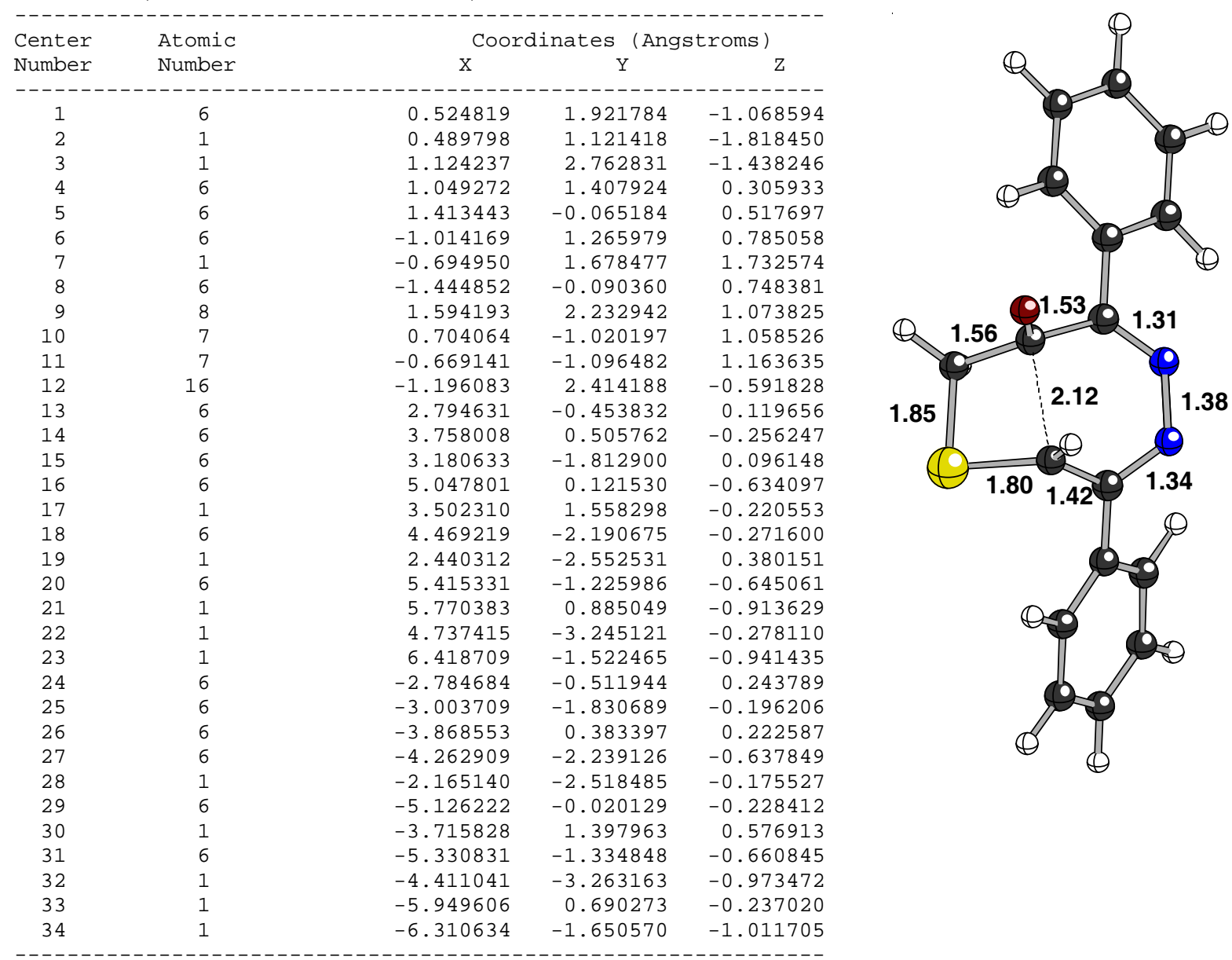




\section{Electrocyclic product, $\mathrm{X}=\mathrm{S}$ (Table 1)}

$\mathrm{HF}=-1238.5831132$ hartrees $(-777223.289364132 \mathrm{kcal} / \mathrm{mol})$

Imaginary Frequencies: none found

Zero-point correction $=0.255911$ (Hartree/Particle)

Temperature 298.150 Kelvin. Pressure 1.00000 Atm.

Sum of electronic and thermal Free Energies $=\mathbf{- 1 2 3 8 . 3 7 4 3 7 0}$ hartrees $(-777092.3009187 \mathrm{kcal} / \mathrm{mol})$

Solvent Single Point: HF = -1238.662579 hartrees $(-777273.1546 \mathrm{kcal} / \mathrm{mol})$

Coordinates (from last standard orientation):

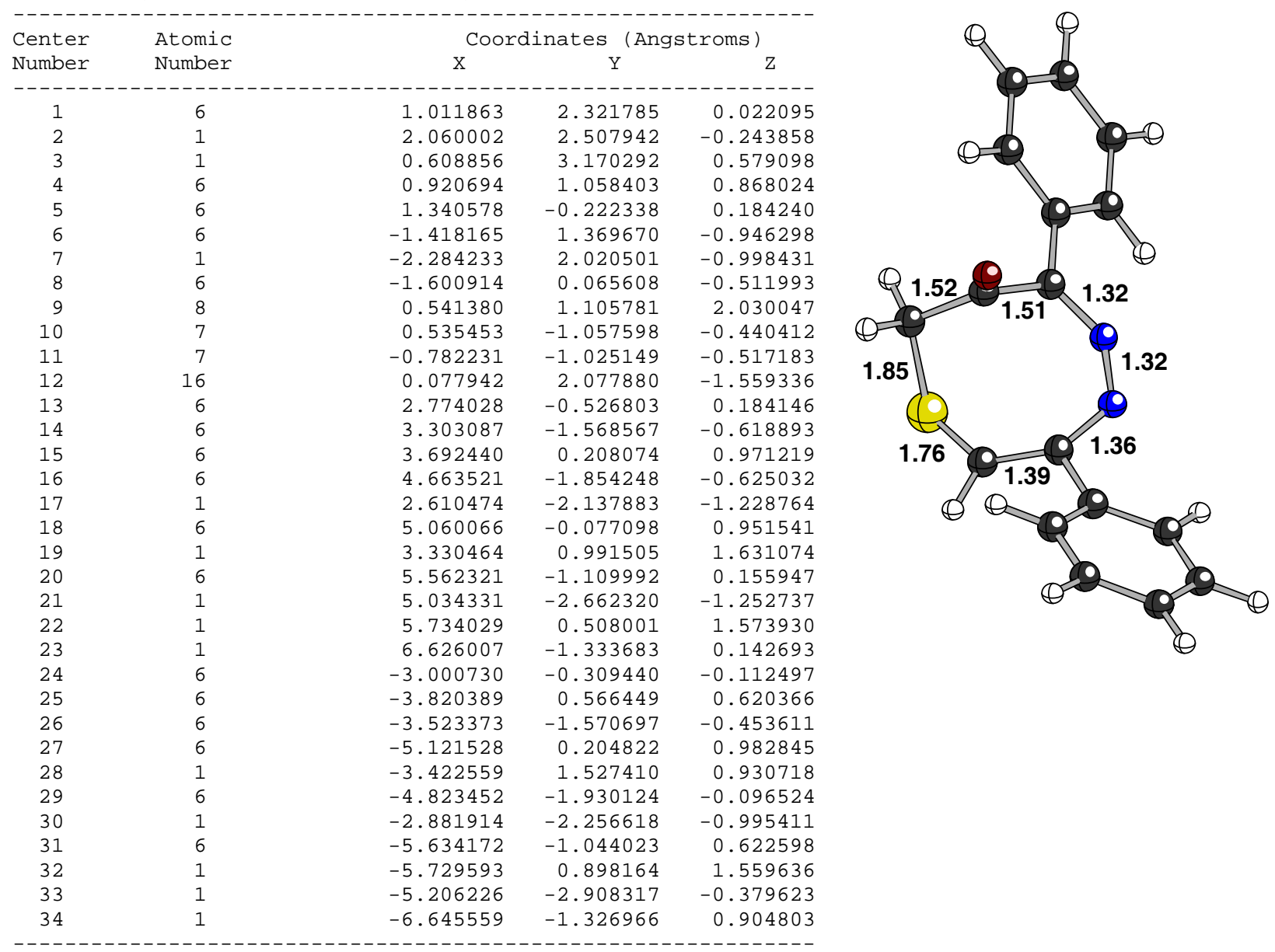




\section{Fragmentation product, $\mathrm{X}=\mathrm{S}$ (Table 1)}

$\mathrm{HF}=-1238.560542$ hartrees $(-777209.12571042 \mathrm{kcal} / \mathrm{mol})$

Imaginary Frequencies: none found

Zero-point correction $=0.256386$ (Hartree/Particle)

Temperature 298.150 Kelvin. Pressure 1.00000 Atm.

Sum of electronic and thermal Free Energies = -1238.350744 hartrees $(-777077.47536744 \mathrm{kcal} / \mathrm{mol})$

Solvent Single Point: HF = -1238.637622 hartrees $(-777257.4939$ kcal/mol)

Coordinates (from last standard orientation):

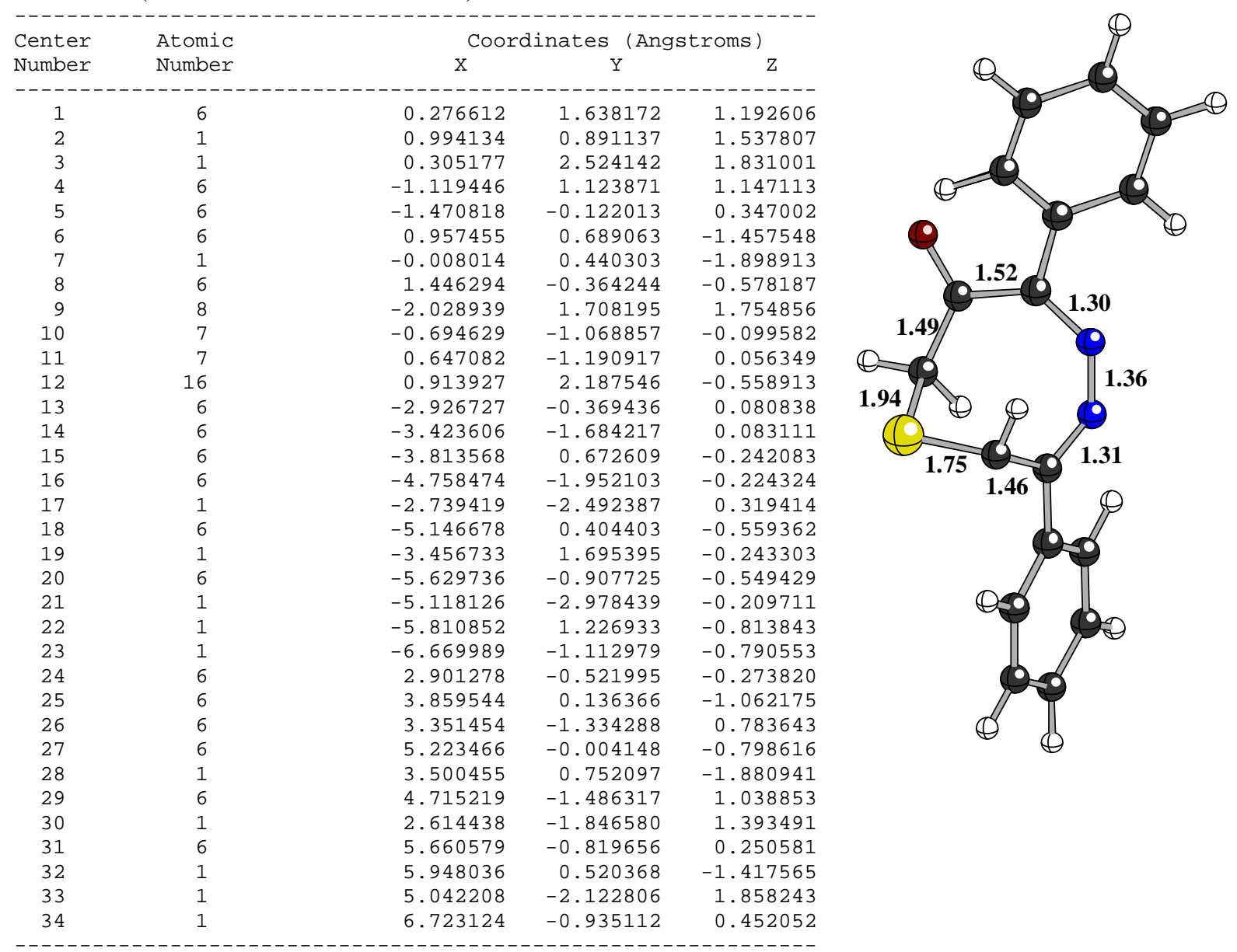




\section{Reactant, $\mathrm{X}=\mathrm{NH}$ (Table 1)}

$\mathrm{HF}=-895.7222041$ hartrees $(-562074.640294791 \mathrm{kcal} / \mathrm{mol})$

Imaginary Frequencies: none found

Zero-point correction $=0.272824$ (Hartree/Particle)

Temperature 298.150 Kelvin. Pressure 1.00000 Atm.

Sum of electronic and thermal Free Energies = $\mathbf{- 8 9 5 . 4 9 4 5 9 6}$ hartrees $(-561931.81393596 \mathrm{kcal} / \mathrm{mol})$

Solvent Single Point: HF $=\mathbf{- 8 9 5 . 8 2 9 7 3 5 3 ~ h a r t r e e s ~}(-562142.1172 \mathrm{kcal} / \mathrm{mol})$

Coordinates (from last standard orientation):

\begin{tabular}{|c|c|c|c|c|}
\hline \multirow{2}{*}{$\begin{array}{l}\text { Center } \\
\text { Number }\end{array}$} & \multirow{2}{*}{$\begin{array}{l}\text { Atomic } \\
\text { Number }\end{array}$} & \multicolumn{3}{|c|}{ Coordinates (Angstroms) } \\
\hline & & $x$ & $Y^{\prime}$ & Z \\
\hline 1 & 6 & $\odot .922975$ & 2.139998 & $-\odot .866586$ \\
\hline 2 & 1 & 1.789191 & 1.988299 & -1.523898 \\
\hline 3 & 1 & $\odot .901039$ & 3.179847 & -0.506893 \\
\hline 4 & 6 & 0.710612 & 1.186157 & $\odot .386885$ \\
\hline 5 & 6 & 1.285563 & -0.208634 & 0.111388 \\
\hline 6 & 6 & -0.806952 & 1.170338 & $-\odot .088454$ \\
\hline 7 & 1 & -1.350780 & 1.924125 & $\odot .497068$ \\
\hline 8 & 6 & -1.473861 & -0.165269 & -0.043806 \\
\hline 9 & 8 & 1.022346 & 1.574732 & 1.613104 \\
\hline 10 & 7 & 0.590492 & -1.302863 & -0.035530 \\
\hline 11 & 7 & $-\odot .805161$ & -1.278700 & $-\odot .037039$ \\
\hline 12 & 7 & -0.365623 & 1.653799 & -1.428900 \\
\hline 13 & 1 & -0.964396 & 2.371764 & -1.830728 \\
\hline 14 & 6 & 2.763904 & $-\odot .388689$ & $\odot .093726$ \\
\hline 15 & 6 & 3.324624 & -1.464648 & -0.622438 \\
\hline 16 & 6 & 3.634654 & 0.515384 & $\odot .733081$ \\
\hline 17 & 6 & 4.707889 & -1.631058 & -0.701262 \\
\hline 18 & 1 & 2.652197 & -2.161146 & -1.111643 \\
\hline 19 & 6 & 5.018588 & $\odot .346921$ & $\odot .647015$ \\
\hline 20 & 1 & 3.185115 & 1.318132 & 1.310942 \\
\hline 21 & 6 & 5.565366 & -0.724914 & $-\odot .067147$ \\
\hline 22 & 1 & 5.118014 & -2.468085 & -1.262523 \\
\hline 23 & 1 & 5.674444 & 1.052425 & 1.152776 \\
\hline 24 & 1 & 6.643852 & $-\odot .854581$ & $-\odot .126751$ \\
\hline 25 & 6 & -2.955083 & $-\odot .287097$ & $\odot .029685$ \\
\hline 26 & 6 & -3.791688 & 0.824344 & -0.180033 \\
\hline 27 & 6 & -3.567225 & -1.529775 & $\odot .295752$ \\
\hline 28 & 6 & -5.183568 & 0.703222 & -0.128078 \\
\hline 29 & 1 & -3.352660 & 1.794119 & -0.390937 \\
\hline 30 & 6 & -4.953303 & -1.650744 & ๑.347301 \\
\hline 31 & 1 & -2.922664 & -2.386834 & 0.456775 \\
\hline 32 & 6 & -5.773937 & $-\odot .534147$ & 0.136267 \\
\hline 33 & 1 & -5.805116 & 1.579699 & $-\odot .295516$ \\
\hline 34 & 1 & $-5.40 \odot 24 \odot$ & -2.619832 & 0.557494 \\
\hline 35 & 1 & -6.856071 & $-\odot .629772$ & 0.179515 \\
\hline
\end{tabular}

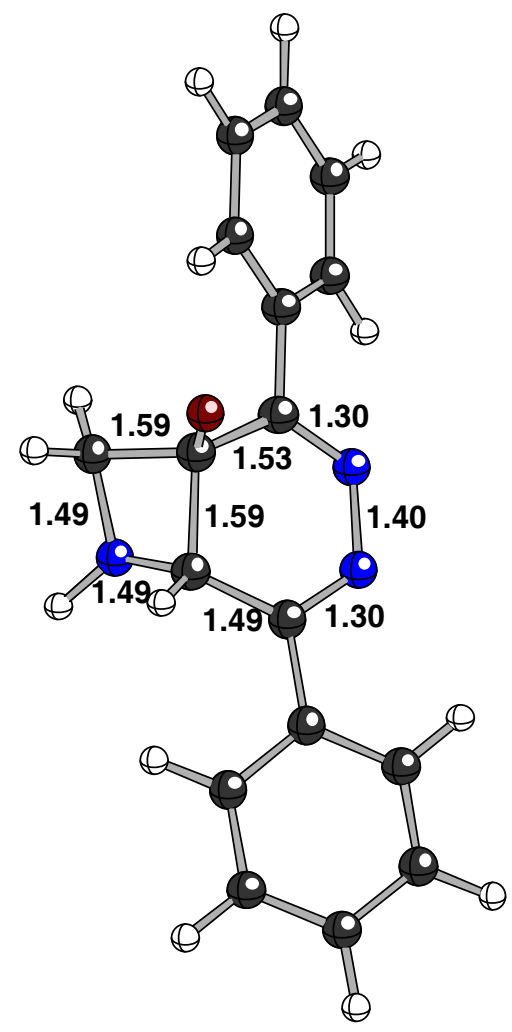




\section{Electrocyclic TS, $X=$ NH (Table 1)}

$\mathrm{HF}=-895.7151165$ hartrees $(-562070.192754915 \mathrm{kcal} / \mathrm{mol})$

Imaginary Frequencies: $1(-310.9291$ 1/cm)

Zero-point correction $=0.271517$ (Hartree/Particle)

Temperature 298.150 Kelvin. Pressure 1.00000 Atm.

Sum of electronic and thermal Free Energies $=\mathbf{- 8 9 5 . 4 8 8 1 7 9}$ hartrees $(-561927.78720429 \mathrm{kcal} / \mathrm{mol})$

Solvent Single Point: HF = $\mathbf{- 8 9 5 . 8 1 4 7 2 3 ~ h a r t r e e s ~}(-\mathbf{5 6 2 1 3 2 . 6 9 6 8 ~} \mathrm{kcal} / \mathrm{mol})$

Coordinates (from last standard orientation):

\begin{tabular}{|c|c|c|c|c|}
\hline \multirow{2}{*}{$\begin{array}{l}\text { Center } \\
\text { Number }\end{array}$} & \multirow{2}{*}{$\begin{array}{l}\text { Atomic } \\
\text { Number }\end{array}$} & \multicolumn{3}{|c|}{ Coordinates (Angstroms) } \\
\hline & & $\mathrm{x}$ & $\mathrm{Y}$ & Z \\
\hline 1 & 6 & 0.924862 & 2.398974 & -0.571402 \\
\hline 2 & 1 & 1.910194 & 2.469448 & -1.058133 \\
\hline 3 & 1 & $\odot .704101$ & 3.349513 & -0.057341 \\
\hline 4 & 6 & 0.767124 & 1.262739 & 0.485432 \\
\hline 5 & 6 & 1.250696 & -0.059276 & -0.107515 \\
\hline 6 & 6 & -0.995116 & 1.359546 & -0.373575 \\
\hline 7 & 1 & -1.592626 & 2.084159 & 0.189537 \\
\hline 8 & 6 & -1.522848 & 0.034263 & -0.486147 \\
\hline 9 & 8 & 0.926088 & 1.485091 & 1.723163 \\
\hline 10 & 7 & 0.573194 & -0.974799 & -0.755911 \\
\hline 11 & 7 & -0.793424 & -1.020274 & -0.817588 \\
\hline 12 & 7 & -0.157577 & 1.915860 & -1.430046 \\
\hline 13 & 1 & -0.626732 & 2.627558 & -1.984997 \\
\hline 14 & 6 & 2.700428 & -0.375445 & 0.058574 \\
\hline 15 & 6 & 3.329642 & -1.248634 & -0.856811 \\
\hline 16 & 6 & 3.497253 & 0.197299 & $1.07120 \odot$ \\
\hline 17 & 6 & 4.690288 & -1.533713 & $-\odot .765009$ \\
\hline 18 & 1 & 2.719861 & -1.693692 & -1.635154 \\
\hline 19 & 6 & 4.863178 & -0.090234 & 1.156673 \\
\hline 20 & 1 & 3.020668 & 0.850911 & 1.793221 \\
\hline 21 & 6 & 5.471767 & -0.956366 & ๑. 244601 \\
\hline 22 & 1 & 5.146444 & -2.207512 & -1.487699 \\
\hline 23 & 1 & 5.451635 & 0.361662 & 1.952593 \\
\hline 24 & 1 & 6.533507 & -1.181057 & 0.318026 \\
\hline 25 & 6 & -2.937046 & -0.265932 & -0.114243 \\
\hline 26 & 6 & -3.939077 & 0.720225 & -0.156309 \\
\hline 27 & 6 & -3.308778 & -1.565983 & ๑. 280901 \\
\hline 28 & 6 & -5.260710 & 0.424235 & 0.188094 \\
\hline 29 & 1 & -3.681622 & 1.725184 & -0.476236 \\
\hline 30 & 6 & -4.628769 & -1.865804 & 0.614729 \\
\hline 31 & 1 & -2.534500 & -2.324808 & 0.312601 \\
\hline 32 & 6 & -5.613955 & -0.871314 & 0.575052 \\
\hline 33 & 1 & -6.015962 & 1.205812 & 0.146623 \\
\hline 34 & 1 & -4.890815 & -2.877014 & 0.917899 \\
\hline 35 & 1 & -6.641834 & -1.103884 & 0.843098 \\
\hline
\end{tabular}

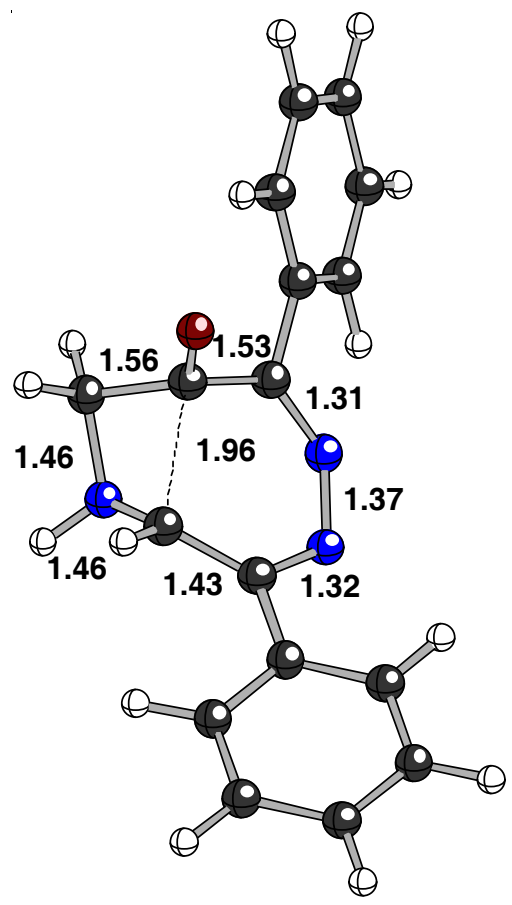




\section{Fragmentation TS, $X=$ NH (Table 1)}

$\mathrm{HF}=-895.6835484$ hartrees $(-562050.383456484 \mathrm{kcal} / \mathrm{mol})$

Imaginary Frequencies: $1(-545.9559$ 1/cm)

Zero-point correction $=0.270421$ (Hartree/Particle)

Temperature 298.150 Kelvin. Pressure 1.00000 Atm.

Sum of electronic and thermal Free Energies $=\mathbf{- 8 9 5 . 4 5 8 6 4 8}$ hartrees $(-561909.25620648 \mathrm{kcal} / \mathrm{mol})$

Solvent Single Point: HF = -895.7737718 hartrees $(-562106.9995 \mathrm{kcal} / \mathrm{mol})$

Coordinates (from last standard orientation):

\begin{tabular}{|c|c|c|c|c|}
\hline \multirow{2}{*}{$\begin{array}{l}\text { Center } \\
\text { Number }\end{array}$} & \multirow{2}{*}{$\begin{array}{l}\text { Atomic } \\
\text { Number }\end{array}$} & \multicolumn{3}{|c|}{ Coordinates (Angstroms) } \\
\hline & & $x$ & $\mathrm{Y}$ & Z \\
\hline 1 & 6 & $\odot .086635$ & 1.921029 & -1.052827 \\
\hline 2 & 1 & -0.108880 & 1.074687 & -1.725111 \\
\hline 3 & 1 & 0.546101 & 2.744673 & -1.622053 \\
\hline 4 & 6 & $\odot .917225$ & 1.485540 & $\odot .194078$ \\
\hline 5 & 6 & 1.348377 & 0.043638 & 0.479561 \\
\hline 6 & 6 & -1.018259 & 1.433981 & 0.789547 \\
\hline 7 & 1 & -0.701099 & 1.853142 & 1.735544 \\
\hline 8 & 6 & -1.479447 & $\odot .090467$ & 0.760176 \\
\hline 9 & 8 & 1.541014 & 2.392472 & $\odot .813796$ \\
\hline 10 & 7 & 0.650344 & -0.870645 & 1.108681 \\
\hline 11 & 7 & -0.721322 & $-\odot .918416$ & 1.217666 \\
\hline 12 & 7 & -1.186685 & 2.303395 & $-\odot .379188$ \\
\hline 13 & 1 & -1.111819 & 3.273875 & -0.082774 \\
\hline 14 & 6 & 2.719768 & $-\odot .350407$ & $\odot .084160$ \\
\hline 15 & 6 & 3.608064 & 0.569476 & -0.514715 \\
\hline 16 & 6 & 3.184453 & -1.671910 & $\odot .281931$ \\
\hline 17 & 6 & 4.897272 & 0.185097 & $-\odot .894056$ \\
\hline 18 & 1 & 3.293124 & 1.597485 & -0.650506 \\
\hline 19 & 6 & 4.472880 & -2.046414 & -0.087205 \\
\hline 20 & 1 & 2.503043 & -2.384088 & 0.734025 \\
\hline 21 & 6 & 5.343027 & -1.122125 & -0.684278 \\
\hline 22 & 1 & 5.558865 & 0.918763 & -1.349875 \\
\hline 23 & 1 & 4.801791 & -3.070105 & 0.080818 \\
\hline 24 & 1 & 6.346082 & -1.419230 & -0.981315 \\
\hline 25 & 6 & -2.799601 & $-\odot .318449$ & $\odot .197798$ \\
\hline 26 & 6 & -3.071341 & -1.674457 & -0.068115 \\
\hline 27 & 6 & -3.824509 & $\odot .619731$ & $-\odot .030521$ \\
\hline 28 & 6 & -4.325487 & -2.078556 & -0.529728 \\
\hline 29 & 1 & -2.278083 & -2.392995 & $\odot .108052$ \\
\hline 30 & 6 & -5.072721 & 0.217766 & -0.506057 \\
\hline 31 & 1 & -3.621674 & 1.668080 & 0.156095 \\
\hline 32 & 6 & -5.333396 & -1.135285 & $-\odot .755810$ \\
\hline 33 & 1 & -4.514912 & -3.132604 & -0.721426 \\
\hline 34 & 1 & -5.846926 & $\odot .961569$ & -0.681184 \\
\hline 35 & 1 & -6.307710 & -1.448107 & -1.124418 \\
\hline
\end{tabular}

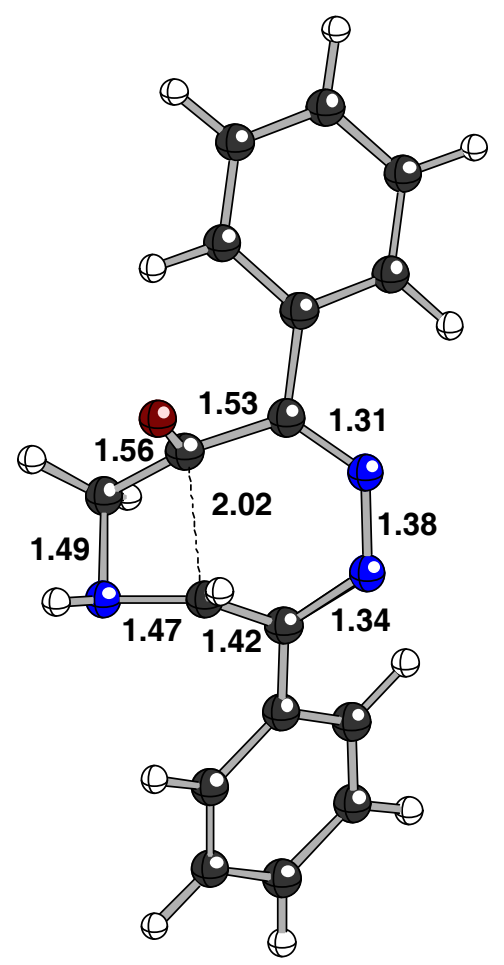




\section{Electrocyclic product, $\mathrm{X}=\mathrm{NH}$ (Table 1)}

$\mathrm{HF}=-895.7390762$ hartrees $(-562085.227706262 \mathrm{kcal} / \mathrm{mol})$

Imaginary Frequencies: none found

Zero-point correction $=0.272175$ (Hartree/Particle)

Temperature 298.150 Kelvin. Pressure 1.00000 Atm.

Sum of electronic and thermal Free Energies $=\mathbf{- 8 9 5 . 5 1 3 6 6 7}$ hartrees $(-561943.78117917 \mathrm{kcal} / \mathrm{mol})$

Solvent Single Point: HF = -895.8285982 hartrees $(-562141.4037 \mathrm{kcal} / \mathrm{mol})$

Coordinates (from last standard orientation):

\begin{tabular}{|c|c|c|c|c|}
\hline \multirow{2}{*}{$\begin{array}{l}\text { Center } \\
\text { Number }\end{array}$} & \multirow{2}{*}{$\begin{array}{l}\text { Atomic } \\
\text { Number }\end{array}$} & \multicolumn{3}{|c|}{ Coordinates (Angstroms) } \\
\hline & & $\mathrm{x}$ & $\mathrm{Y}$ & Z \\
\hline 1 & 6 & 0.448774 & 2.667074 & -0.422749 \\
\hline 2 & 1 & 1.303538 & 3.173086 & -0.896350 \\
\hline 3 & 1 & $-\odot .001276$ & 3.380911 & $\odot .283506$ \\
\hline 4 & 6 & 1.095983 & 1.565422 & 0.436857 \\
\hline 5 & 6 & 1.368577 & 0.233334 & -0.103102 \\
\hline 6 & 6 & -1.653488 & 1.631400 & -1.004067 \\
\hline 7 & 1 & -2.581806 & 2.207116 & -1.013563 \\
\hline 8 & 6 & -1.675675 & $\odot .321426$ & -0.630099 \\
\hline 9 & 8 & 1.581072 & 1.993285 & 1.502204 \\
\hline 10 & 7 & 0.563494 & -0.581158 & -0.831034 \\
\hline 11 & 7 & -0.712367 & -0.641385 & $-\odot .962196$ \\
\hline 12 & 7 & -0.483116 & 2.261789 & -1.467227 \\
\hline 13 & 1 & -0.690283 & 3.042096 & -2.079411 \\
\hline 14 & 6 & 2.731369 & $-\odot .343967$ & $\odot .082626$ \\
\hline 15 & 6 & 3.114499 & -1.497295 & -0.652793 \\
\hline 16 & 6 & 3.719416 & 0.205018 & $\odot .937437$ \\
\hline 17 & 6 & 4.390726 & -2.044648 & $-\odot .555003$ \\
\hline 18 & 1 & 2.377132 & -1.947157 & -1.306504 \\
\hline 19 & 6 & 4.999133 & $-\odot .350585$ & 1.029512 \\
\hline 20 & 1 & 3.468198 & 1.064177 & 1.544476 \\
\hline 21 & 6 & 5.356807 & -1.479615 & 0.288765 \\
\hline 22 & 1 & 4.633648 & -2.926881 & -1.145412 \\
\hline 23 & 1 & 5.721177 & 0.107850 & 1.703459 \\
\hline 24 & 1 & 6.351293 & -1.912401 & $\odot .370546$ \\
\hline 25 & 6 & -2.937581 & $-\odot .262262$ & -0.101513 \\
\hline 26 & 6 & -3.820468 & 0.490184 & $\odot .697093$ \\
\hline 27 & 6 & -3.284176 & -1.599650 & $-\odot .381926$ \\
\hline 28 & 6 & -5.017912 & -0.057220 & 1.165852 \\
\hline 29 & 1 & -3.548406 & 1.502844 & $\odot .978428$ \\
\hline 30 & 6 & -4.476323 & -2.146566 & $\odot .092299$ \\
\hline 31 & 1 & -2.593810 & -2.187259 & -0.976487 \\
\hline 32 & 6 & -5.356750 & -1.379172 & 0.864631 \\
\hline 33 & 1 & -5.676715 & $\odot .546618$ & 1.785991 \\
\hline 34 & 1 & -4.721373 & -3.180026 & -0.142870 \\
\hline 35 & 1 & -6.283440 & -1.809256 & 1.236851 \\
\hline
\end{tabular}

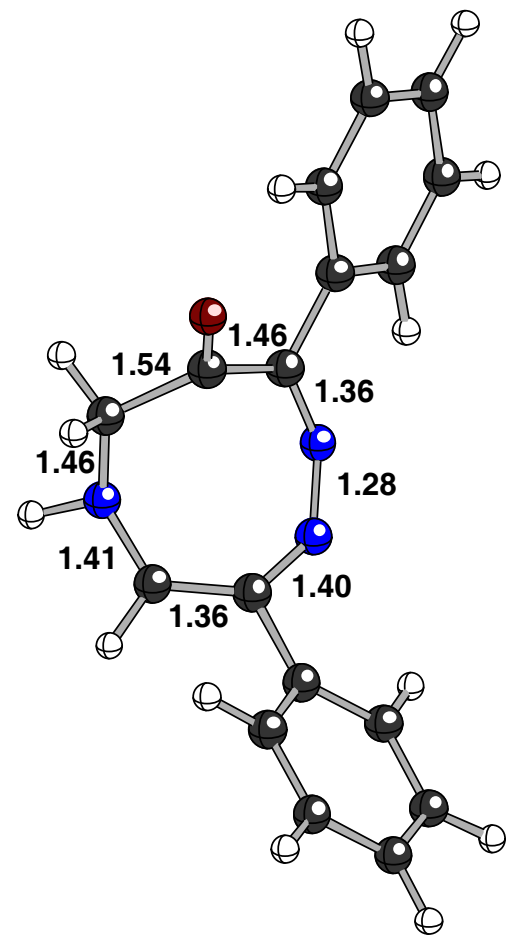




\section{Fragmentation product, $\mathrm{X}=\mathrm{NH}$ (Table 1)}

$\mathrm{HF}=-895.7095642$ hartrees $(-562066.708631142 \mathrm{kcal} / \mathrm{mol})$

Imaginary Frequencies: none found

Zero-point correction $=0.272526$ (Hartree/Particle)

Temperature 298.150 Kelvin. Pressure 1.00000 Atm.

Sum of electronic and thermal Free Energies $=\mathbf{- 8 9 5 . 4 8 2 7 6 7}$ hartrees $(-561924.39112017 \mathrm{kcal} / \mathrm{mol})$

Solvent Single Point: HF = -895.7991577 hartrees $(-562122.9294 \mathrm{kcal} / \mathrm{mol})$

Coordinates (from last standard orientation):

\begin{tabular}{|c|c|c|c|c|}
\hline \multirow{2}{*}{$\begin{array}{l}\text { Center } \\
\text { Number }\end{array}$} & \multirow{2}{*}{$\begin{array}{l}\text { Atomic } \\
\text { Number }\end{array}$} & \multicolumn{3}{|c|}{ Coordinates (Angstroms) } \\
\hline & & $\mathrm{x}$ & $\mathrm{Y}$ & Z \\
\hline 1 & 6 & -0.643134 & 2.055314 & 0.144547 \\
\hline 2 & 1 & -1.561091 & 1.990400 & -0.450408 \\
\hline 3 & 1 & $-\odot .225706$ & 3.051890 & -0.041517 \\
\hline 4 & 6 & 0.392177 & 1.055359 & -0.490780 \\
\hline 5 & 6 & 1.125445 & -0.090105 & 0.130071 \\
\hline 6 & 6 & -0.928329 & $\odot .595374$ & 1.967354 \\
\hline 7 & 1 & -0.218524 & 0.328813 & 2.751889 \\
\hline 8 & 6 & -1.400120 & -0.420123 & 1.179633 \\
\hline 9 & 8 & 0.660362 & 1.412336 & -1.651452 \\
\hline 10 & 7 & 0.668478 & -1.229161 & 0.746416 \\
\hline 11 & 7 & $-\odot .52419 \odot$ & -1.544956 & 1.167023 \\
\hline 12 & 7 & $-\odot .952574$ & 1.923641 & 1.574654 \\
\hline 13 & 1 & -0.386897 & 2.519752 & 2.164264 \\
\hline 14 & 6 & 2.592972 & $-\odot .179632$ & $-\odot .137561$ \\
\hline 15 & 6 & $3.37 \odot 222$ & -1.123745 & $\odot .586176$ \\
\hline 16 & 6 & 3.315454 & 0.667394 & -1.015336 \\
\hline 17 & 6 & 4.752032 & -1.217645 & 0.438925 \\
\hline 18 & 1 & 2.853795 & -1.787952 & 1.268670 \\
\hline 19 & 6 & 4.703599 & $\odot .576281$ & -1.149829 \\
\hline 20 & 1 & 2.776582 & 1.385912 & -1.617386 \\
\hline 21 & 6 & 5.444392 & -0.365915 & -0.431199 \\
\hline 22 & 1 & 5.295377 & -1.962636 & 1.018209 \\
\hline 23 & 1 & 5.206691 & 1.248363 & -1.843287 \\
\hline 24 & 1 & 6.523451 & $-\odot .437937$ & $-\odot .546343$ \\
\hline 25 & 6 & -2.583933 & $-\odot .429783$ & $\odot .319343$ \\
\hline 26 & 6 & -3.603211 & 0.547053 & 0.411216 \\
\hline 27 & 6 & -2.772975 & -1.486003 & $-\odot .600 \odot 52$ \\
\hline 28 & 6 & -4.750782 & 0.467624 & -0.375443 \\
\hline 29 & 1 & -3.488070 & 1.365670 & 1.115651 \\
\hline 30 & 6 & -3.926244 & -1.563810 & -1.380321 \\
\hline 31 & 1 & -1.995999 & -2.238988 & -0.678595 \\
\hline 32 & 6 & -4.925278 & $-\odot .58906 \odot$ & -1.278263 \\
\hline 33 & 1 & -5.516826 & 1.234761 & $-\odot .282603$ \\
\hline 34 & 1 & -4.041514 & -2.387658 & -2.081233 \\
\hline 35 & 1 & -5.819883 & $-\odot .647201$ & -1.893219 \\
\hline
\end{tabular}

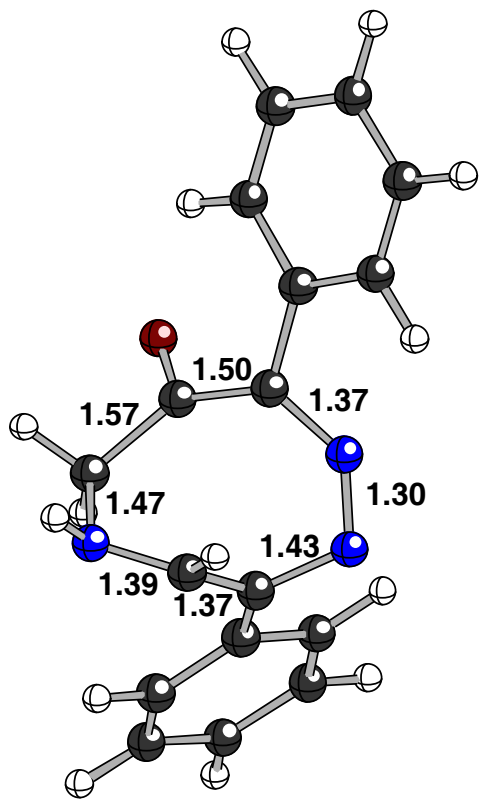




\section{8 (Figure 4)}

$\mathrm{HF}=-958.3453245$ hartrees $(-601371.274576995 \mathrm{kcal} / \mathrm{mol})$

Imaginary Frequencies: none found

Zero-point correction $=0.342536$ (Hartree/Particle)

Temperature 298.150 Kelvin. Pressure 1.00000 Atm.

Sum of electronic and thermal Free Energies = -958.049744 hartrees $\mathbf{( - 6 0 1 1 8 5 . 7 9 4 8 5 7 4 4 ~} \mathbf{k c a l} / \mathrm{mol})$

Solvent Single Point: HF $=\mathbf{- 9 5 8 . 4 3 8 9 5 3 6}$ hartrees $(-601430.0278 \mathrm{kcal} / \mathrm{mol})$

Coordinates (from last standard orientation):

\begin{tabular}{|c|c|c|c|c|}
\hline \multirow{2}{*}{$\begin{array}{l}\text { Center } \\
\text { Number }\end{array}$} & \multirow{2}{*}{$\begin{array}{l}\text { Atomic } \\
\text { Number }\end{array}$} & \multicolumn{3}{|c|}{ Coordinates (Angstroms) } \\
\hline & & $\mathrm{X}$ & $\mathrm{Y}$ & Z \\
\hline \multicolumn{5}{|c|}{ 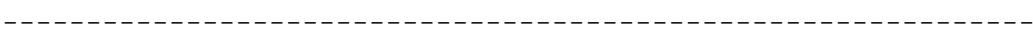 } \\
\hline 1 & 6 & $-\odot .781860$ & $\odot .998712$ & -0.114234 \\
\hline 2 & 6 & -1.467881 & $-\odot .397385$ & -0.051891 \\
\hline 3 & 6 & 0.625090 & 0.737491 & -0.795935 \\
\hline 4 & 1 & 0.390587 & 0.552370 & -1.858097 \\
\hline 5 & 6 & 1.276139 & -0.512068 & -0.234466 \\
\hline 6 & 8 & -1.455467 & 1.938841 & -0.803898 \\
\hline 7 & 7 & -0.811444 & -1.516849 & ๑. .054301 \\
\hline 8 & 7 & 0.581030 & -1.508801 & 0.212685 \\
\hline 9 & 6 & 2.753680 & $-\odot .719903$ & -0.184052 \\
\hline 10 & 6 & 3.348674 & -1.317857 & ๑. 941814 \\
\hline 11 & 6 & 3.581615 & -0.378599 & -1.267861 \\
\hline 12 & 6 & 4.723052 & -1.550908 & ๑. 989055 \\
\hline 13 & 1 & 2.709905 & -1.601839 & 1.771612 \\
\hline 14 & 6 & 4.956802 & -0.623250 & -1.226918 \\
\hline 15 & 1 & 3.142523 & ๑. 059626 & -2.158618 \\
\hline 16 & 6 & 5.536818 & -1.203583 & -0.095498 \\
\hline 17 & 1 & 5.161484 & -2.006584 & 1.873804 \\
\hline 18 & 1 & 5.574775 & $-\odot .360616$ & -2.082218 \\
\hline 19 & 1 & 6.608054 & -1.385260 & -0.059504 \\
\hline 20 & 6 & -2.950478 & $-\odot .555242$ & -0.089644 \\
\hline 21 & 6 & -3.539499 & -1.702216 & ๑. 482855 \\
\hline 22 & 6 & -3.792093 & 0.407571 & -0.682640 \\
\hline 23 & 6 & -4.920996 & -1.890376 & ๑. 453931 \\
\hline 24 & 1 & -2.890453 & -2.437822 & ๑. 945448 \\
\hline 25 & 6 & -5.176732 & ๑. 212800 & -0.704937 \\
\hline 26 & 1 & -3.302594 & 1.289268 & -1.090783 \\
\hline 27 & 6 & -5.749281 & -0.932299 & -0.143563 \\
\hline 28 & 1 & -5.353627 & -2.781823 & ๑. 903350 \\
\hline 29 & 1 & -5.812502 & ๑. 964947 & -1.167561 \\
\hline 30 & 1 & -6.827691 & -1.076690 & -0.164930 \\
\hline 31 & 6 & 1.445231 & 2.040794 & -0.706298 \\
\hline 32 & 6 & -0.539109 & 1.424031 & 1.387400 \\
\hline 33 & 6 & 1.638584 & 2.530648 & ๑.738488 \\
\hline 34 & 1 & 2.417995 & 1.944640 & -1.201444 \\
\hline 35 & 1 & ๑. 861234 & 2.787229 & -1.255922 \\
\hline 36 & 6 & ๑. 287035 & 2.714005 & 1. 446191 \\
\hline 37 & 1 & -0.035395 & 0.629129 & 1.960381 \\
\hline 38 & 1 & -1.527384 & 1.582228 & 1.835579 \\
\hline 39 & 1 & 2.198954 & 3.476949 & 0.731941 \\
\hline 40 & 1 & 2.253732 & 1.810269 & 1. 299321 \\
\hline 41 & 1 & 0.442089 & 3.027175 & 2.489339 \\
\hline 42 & 1 & -0.283795 & 3.501431 & ๑. 939794 \\
\hline
\end{tabular}

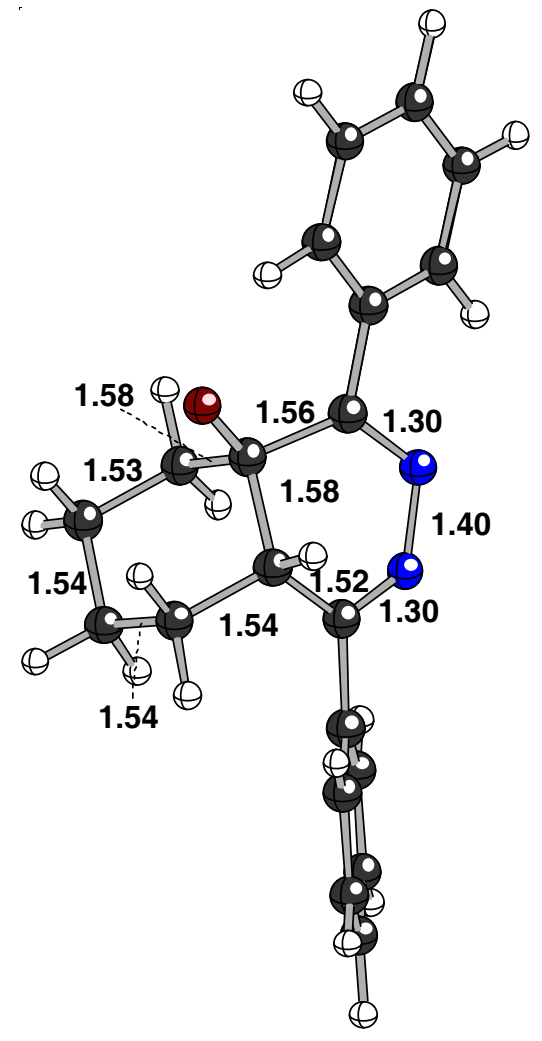




\section{TS8 $\rightarrow 9$ (Figure 4)}

$\mathrm{HF}=-958.3065158$ hartrees $(-601346.921729658 \mathrm{kcal} / \mathrm{mol})$

Imaginary Frequencies: 1 (-250.7803 1/cm)

Zero-point correction $=0.340117$ (Hartree/Particle)

Temperature 298.150 Kelvin. Pressure 1.00000 Atm.

Sum of electronic and thermal Free Energies = -958.013619 hartrees $\mathbf{( - 6 0 1 1 6 3 . 1 2 6 0 5 8 6 9} \mathbf{~ k c a l} / \mathbf{m o l})$

Solvent Single Point: HF = -958.3835498 hartrees $(-601395.2613 \mathrm{kcal} / \mathrm{mol})$

Coordinates (from last standard orientation):

\begin{tabular}{|c|c|c|c|c|}
\hline \multirow{2}{*}{$\begin{array}{l}\text { Center } \\
\text { Number }\end{array}$} & \multirow{2}{*}{$\begin{array}{l}\text { Atomic } \\
\text { Number }\end{array}$} & \multicolumn{3}{|c|}{ Coordinates (Angstroms) } \\
\hline & & $\mathrm{x}$ & $\mathrm{Y}$ & Z \\
\hline 1 & 6 & 1.211354 & 1.545909 & -0.260400 \\
\hline 2 & 6 & 1.454594 & 0.130297 & -0.584054 \\
\hline 3 & 6 & -0.457630 & 0.531378 & 0.788912 \\
\hline 4 & 1 & -0.144864 & -0.077422 & 1.650833 \\
\hline 5 & 6 & -1.213098 & -0.221445 & -0.240933 \\
\hline 6 & 8 & 1.889021 & 2.211014 & 0.573522 \\
\hline 7 & 7 & 0.695021 & -0.570463 & -1.448728 \\
\hline 8 & 7 & -0.633753 & -0.576535 & -1.390886 \\
\hline 9 & 6 & -2.555638 & -0.759942 & -0.007430 \\
\hline 10 & 6 & -3.326800 & -1.320305 & -1.060921 \\
\hline 11 & 6 & -3.151515 & -0.737735 & 1.276868 \\
\hline 12 & 6 & -4.607778 & -1.810279 & -0.839194 \\
\hline 13 & 1 & -2.882837 & -1.360481 & -2.049541 \\
\hline 14 & 6 & -4.432271 & -1.252620 & 1.496915 \\
\hline 15 & 1 & -2.598413 & -0.324358 & 2.113301 \\
\hline 16 & 6 & -5.178909 & -1.787491 & 0.444759 \\
\hline 17 & 1 & -5.170840 & -2.224733 & -1.673492 \\
\hline 18 & 1 & -4.849138 & -1.227829 & 2.501917 \\
\hline 19 & 1 & -6.179906 & -2.176050 & 0.614358 \\
\hline 20 & 6 & 2.702831 & -0.573624 & -0.152149 \\
\hline 21 & 6 & 2.856702 & -1.940095 & -0.471257 \\
\hline 22 & 6 & 3.744897 & 0.060057 & $\odot .555773$ \\
\hline 23 & 6 & 4.006914 & -2.640888 & -0.113507 \\
\hline 24 & 1 & 2.055690 & -2.426342 & -1.017244 \\
\hline 25 & 6 & 4.894865 & -0.649121 & 0.915630 \\
\hline 26 & 1 & 3.619317 & 1.101401 & $\odot .82490 \odot$ \\
\hline 27 & 6 & 5.036589 & -1.999243 & 0.585199 \\
\hline 28 & 1 & 4.099121 & -3.692409 & -0.376665 \\
\hline 29 & 1 & 5.684664 & -0.138168 & 1.462128 \\
\hline 30 & 1 & 5.932454 & -2.546275 & ๑.870871 \\
\hline 31 & 6 & -0.970986 & 1.875769 & 1.293182 \\
\hline 32 & 6 & 0.417877 & 2.358282 & -1.305606 \\
\hline 33 & 6 & -1.526028 & 2.854535 & 0.244834 \\
\hline 34 & 1 & -1.774160 & 1.677717 & 2.024864 \\
\hline 35 & 1 & $-\odot .157781$ & 2.363839 & 1.850208 \\
\hline 36 & 6 & -0.435241 & 3.448595 & -0.654819 \\
\hline 37 & 1 & -0.191950 & 1.723808 & -1.950856 \\
\hline 38 & 1 & 1.189987 & 2.819536 & -1.939063 \\
\hline 39 & 1 & -2.048411 & 3.667559 & 0.768871 \\
\hline 40 & 1 & -2.278638 & 2.340987 & $-\odot .368807$ \\
\hline 41 & 1 & -0.891331 & 4.077115 & -1.432663 \\
\hline 42 & 1 & 0.221635 & 4.090778 & $-\odot .055608$ \\
\hline
\end{tabular}

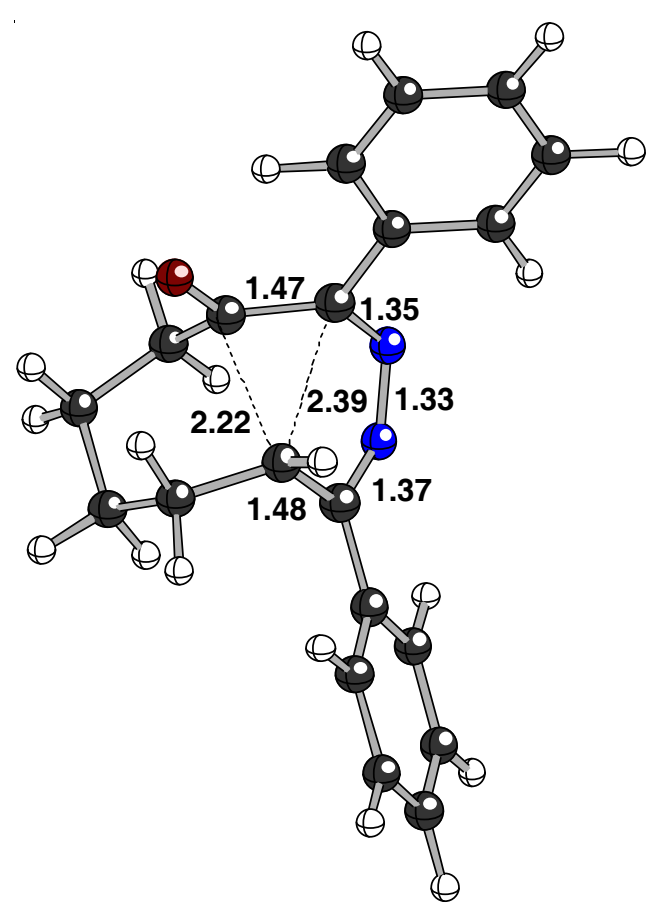




\section{9 (Figure 4)}

$\mathrm{HF}=-958.3657059$ hartrees $(-601384.064109309 \mathrm{kcal} / \mathrm{mol})$

Imaginary Frequencies: none found

Zero-point correction $=0.342883$ (Hartree/Particle)

Temperature 298.150 Kelvin. Pressure 1.00000 Atm.

Sum of electronic and thermal Free Energies $=\mathbf{- 9 5 8 . 0 7 0 6 2 7}$ hartrees $(-601198.89914877 \mathrm{kcal} / \mathrm{mol})$

Solvent Single Point: HF = -958.4476732 hartrees $(-601435.4994 \mathrm{kcal} / \mathrm{mol})$

Coordinates (from last standard orientation):

\begin{tabular}{|c|c|c|c|c|}
\hline \multirow{2}{*}{$\begin{array}{l}\text { Center } \\
\text { Number }\end{array}$} & \multirow{2}{*}{$\begin{array}{l}\text { Atomic } \\
\text { Number }\end{array}$} & \multicolumn{3}{|c|}{ Coordinates (Angstroms) } \\
\hline & & $\mathrm{x}$ & $\mathrm{Y}$ & Z \\
\hline 1 & 6 & 1.555119 & 1.732306 & -0.669455 \\
\hline 2 & 6 & 1.138950 & 0.263346 & -0.462991 \\
\hline 3 & 6 & -0.060557 & 0.074242 & 0.544634 \\
\hline 4 & 1 & 0.148934 & -0.830280 & 1.139958 \\
\hline 5 & 6 & -1.187535 & -0.215623 & -0.446821 \\
\hline 6 & 8 & 2.723593 & 2.103710 & -0.637916 \\
\hline 7 & 7 & 0.574882 & -0.084195 & -1.822272 \\
\hline 8 & 7 & -0.707721 & -0.296625 & -1.694754 \\
\hline 9 & 6 & -2.539782 & -0.607865 & -0.145455 \\
\hline 10 & 6 & -3.494500 & -0.835797 & -1.181212 \\
\hline 11 & 6 & -3.001538 & -0.806218 & 1.185040 \\
\hline 12 & 6 & -4.797846 & -1.220438 & -0.899497 \\
\hline 13 & 1 & -3.170249 & -0.701843 & -2.207851 \\
\hline 14 & 6 & -4.314914 & -1.194546 & 1.457027 \\
\hline 15 & 1 & -2.317314 & -0.668648 & 2.015779 \\
\hline 16 & 6 & -5.234974 & -1.404000 & 0.425375 \\
\hline 17 & 1 & -5.491771 & -1.381264 & -1.723122 \\
\hline 18 & 1 & -4.619354 & -1.337766 & 2.492655 \\
\hline 19 & 1 & -6.258224 & -1.700897 & 0.640181 \\
\hline 20 & 6 & 2.324254 & -0.624510 & -0.101102 \\
\hline 21 & 6 & 2.591467 & -1.788326 & -0.834371 \\
\hline 22 & 6 & 3.136772 & -0.341120 & 1.009576 \\
\hline 23 & 6 & 3.631881 & -2.648308 & -0.466140 \\
\hline 24 & 1 & 1.970065 & -1.997275 & -1.698730 \\
\hline 25 & 6 & 4.174751 & -1.197702 & 1.381443 \\
\hline 26 & 1 & 2.966718 & 0.565026 & 1.582278 \\
\hline 27 & 6 & 4.428983 & -2.359258 & 0.643995 \\
\hline 28 & 1 & 3.817084 & -3.547228 & -1.050226 \\
\hline 29 & 1 & 4.791156 & -0.953774 & 2.243976 \\
\hline 30 & 1 & 5.238288 & -3.026713 & 0.931319 \\
\hline 31 & 6 & -0.278946 & 1.200008 & 1.596571 \\
\hline 32 & 6 & 0.457335 & 2.740661 & -0.995972 \\
\hline 33 & 6 & -0.968021 & 2.492897 & 1.127159 \\
\hline 34 & 1 & -0.880355 & 0.781528 & 2.411915 \\
\hline 35 & 1 & 0.692018 & 1.447460 & 2.052061 \\
\hline 36 & 6 & -0.092752 & 3.418759 & 0.274660 \\
\hline 37 & 1 & -0.358876 & 2.257440 & -1.538706 \\
\hline 38 & 1 & 0.916496 & 3.492594 & -1.646713 \\
\hline 39 & 1 & -1.306463 & 3.048305 & 2.013568 \\
\hline $4 \odot$ & 1 & -1.869258 & 2.223641 & 0.562038 \\
\hline 41 & 1 & -0.684267 & 4.294877 & $-\odot .025420$ \\
\hline 42 & 1 & 0.747630 & 3.799814 & 0.872763 \\
\hline
\end{tabular}

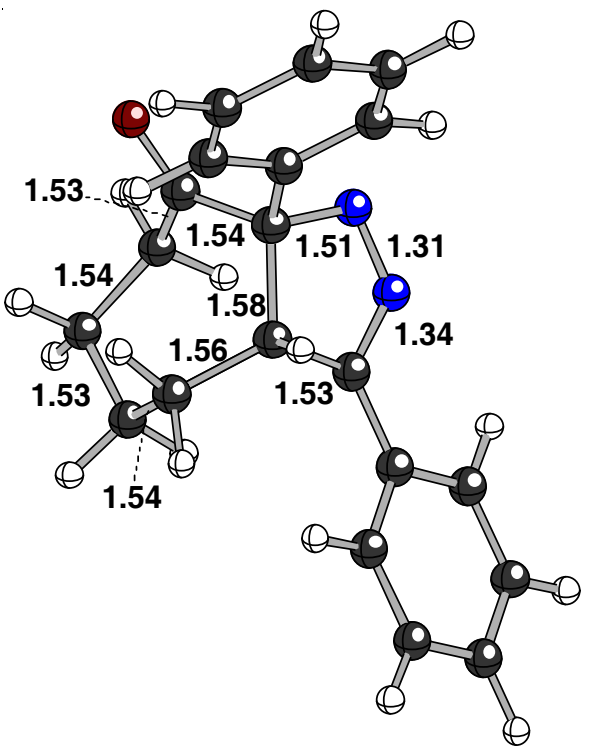




\section{0 (Figure 4)}

$\mathrm{HF}=-997.6492986$ hartrees $(-626034.911364486 \mathrm{kcal} / \mathrm{mol})$

Imaginary Frequencies: none found

Zero-point correction $=0.370942$ (Hartree/Particle)

Temperature 298.150 Kelvin. Pressure 1.00000 Atm.

Sum of electronic and thermal Free Energies $=\mathbf{- 9 9 7 . 3 2 7 6 5 7}$ hartrees $(-625833.07804407 \mathrm{kcal} / \mathrm{mol})$

Solvent Single Point: HF = -997.7399346 hartrees $(-626091.7864 \mathrm{kcal} / \mathrm{mol})$

Coordinates (from last standard orientation):

\begin{tabular}{|c|c|c|c|c|}
\hline \multirow{2}{*}{$\begin{array}{l}\text { Center } \\
\text { Number }\end{array}$} & \multirow{2}{*}{$\begin{array}{l}\text { Atomic } \\
\text { Number }\end{array}$} & \multicolumn{3}{|c|}{ Coordinates (Angstroms) } \\
\hline & & $\mathrm{x}$ & $\mathrm{Y}$ & Z \\
\hline 1 & 6 & -0.770033 & $\odot .819984$ & -0.173809 \\
\hline 2 & 6 & -1.503415 & -0.566416 & $-\odot .079178$ \\
\hline 3 & 6 & 0.569103 & 0.445101 & -0.951328 \\
\hline 4 & 1 & 0.199018 & 0.112190 & -1.936744 \\
\hline 5 & 6 & 1.222634 & -0.756817 & -0.298427 \\
\hline 6 & 8 & -1.449844 & 1.768401 & -0.837463 \\
\hline 7 & 7 & -0.879739 & -1.703066 & 0.027075 \\
\hline 8 & 7 & 0.512560 & -1.715722 & 0.202801 \\
\hline 9 & 6 & 2.698250 & -0.951184 & -0.158980 \\
\hline 10 & 6 & 3.240663 & -1.336341 & 1.079797 \\
\hline 11 & 6 & 3.572184 & -0.816207 & -1.251188 \\
\hline 12 & 6 & 4.610338 & -1.559129 & 1.227020 \\
\hline 13 & 1 & 2.566936 & -1.463761 & 1.920820 \\
\hline 14 & 6 & 4.942416 & -1.049538 & -1.108521 \\
\hline 15 & 1 & 3.173769 & -0.550873 & -2.225200 \\
\hline 16 & 6 & 5.470399 & -1.414796 & 0.133145 \\
\hline 17 & 1 & 5.007451 & -1.847703 & 2.197333 \\
\hline 18 & 1 & 5.597185 & -0.948776 & -1.970781 \\
\hline 19 & 1 & 6.537661 & -1.587963 & 0.246271 \\
\hline 20 & 6 & -2.990756 & -0.678948 & -0.084321 \\
\hline 21 & 6 & -3.601203 & -1.780878 & 0.549810 \\
\hline 22 & 6 & -3.815663 & 0.279275 & -0.707169 \\
\hline 23 & 6 & -4.988092 & -1.930995 & 0.552497 \\
\hline 24 & 1 & -2.964844 & -2.513023 & 1.035128 \\
\hline 25 & 6 & -5.204914 & 0.122717 & $-\odot .699186$ \\
\hline 26 & 1 & -3.312609 & 1.130362 & -1.159523 \\
\hline 27 & 6 & -5.799431 & -0.978473 & $-\odot .07519 \odot$ \\
\hline 28 & 1 & -5.437094 & -2.788204 & 1.049887 \\
\hline 29 & 1 & -5.827714 & 0.870589 & -1.185731 \\
\hline 30 & 1 & -6.881677 & -1.092111 & -0.071783 \\
\hline 31 & 6 & 1.442387 & 1.683375 & -1.207394 \\
\hline 32 & 6 & -0.462408 & 1.223242 & 1.323833 \\
\hline 33 & 6 & 1.941505 & 2.470791 & $\odot .022046$ \\
\hline 34 & 6 & -0.193508 & 2.721946 & 1.539366 \\
\hline 35 & 6 & 0.875455 & 3.391877 & 0.643467 \\
\hline 36 & 1 & -1.348128 & ๑.970801 & 1.923332 \\
\hline 37 & 1 & 0.361635 & 0.623006 & 1.736359 \\
\hline 38 & 1 & -1.137997 & 3.254821 & 1.391263 \\
\hline 39 & 1 & 0.085491 & 2.851795 & 2.596781 \\
\hline $4 \odot$ & 1 & 0.363305 & 3.899071 & -0.181718 \\
\hline 41 & 1 & 1.384334 & 4.172502 & 1.229715 \\
\hline 42 & 1 & 2.361333 & 1.793838 & 0.779313 \\
\hline 43 & 1 & 2.779932 & 3.101374 & -0.309051 \\
\hline 44 & 1 & 0.819753 & 2.355999 & -1.808461 \\
\hline 45 & 1 & 2.305812 & 1.408866 & -1.824370 \\
\hline
\end{tabular}

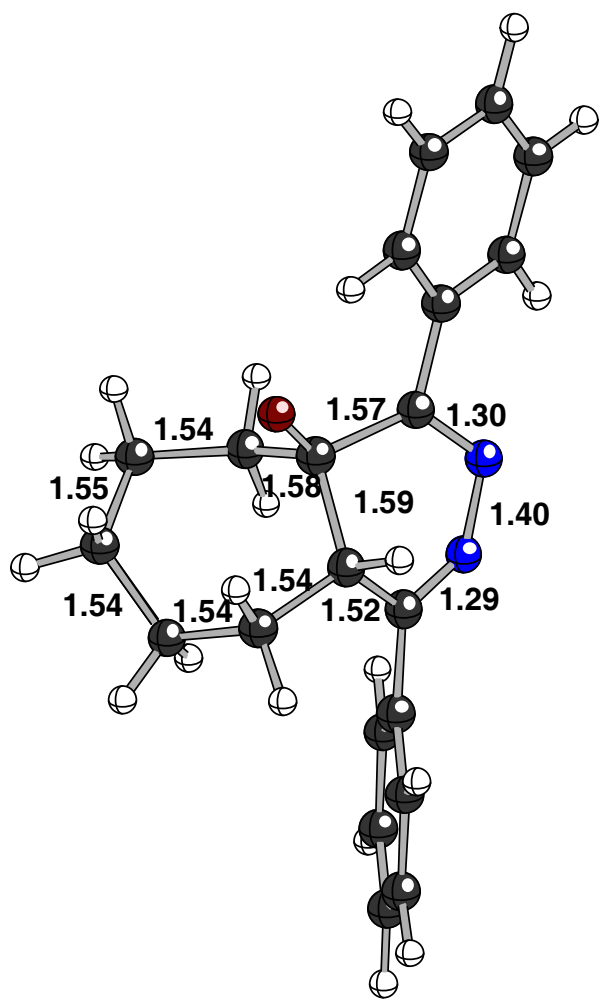




\section{TS10 $\rightarrow 11$ (Figure 4)}

$\mathrm{HF}=-997.6097383$ hartrees $(-626010.086880633 \mathrm{kcal} / \mathrm{mol})$

Imaginary Frequencies: $1(-329.3166$ 1/cm)

Zero-point correction $=0.368704$ (Hartree/Particle)

Temperature 298.150 Kelvin. Pressure 1.00000 Atm.

Sum of electronic and thermal Free Energies $=\mathbf{- 9 9 7 . 2 8 9 4 0 5}$ hartrees $(-625809.07453155 \mathrm{kcal} / \mathrm{mol})$

Solvent Single Point: HF = $\mathbf{- 9 9 7 . 6 8 5 9 2 8 9}$ hartrees $(-626057.8972 \mathrm{kcal} / \mathrm{mol})$

Coordinates (from last standard orientation):

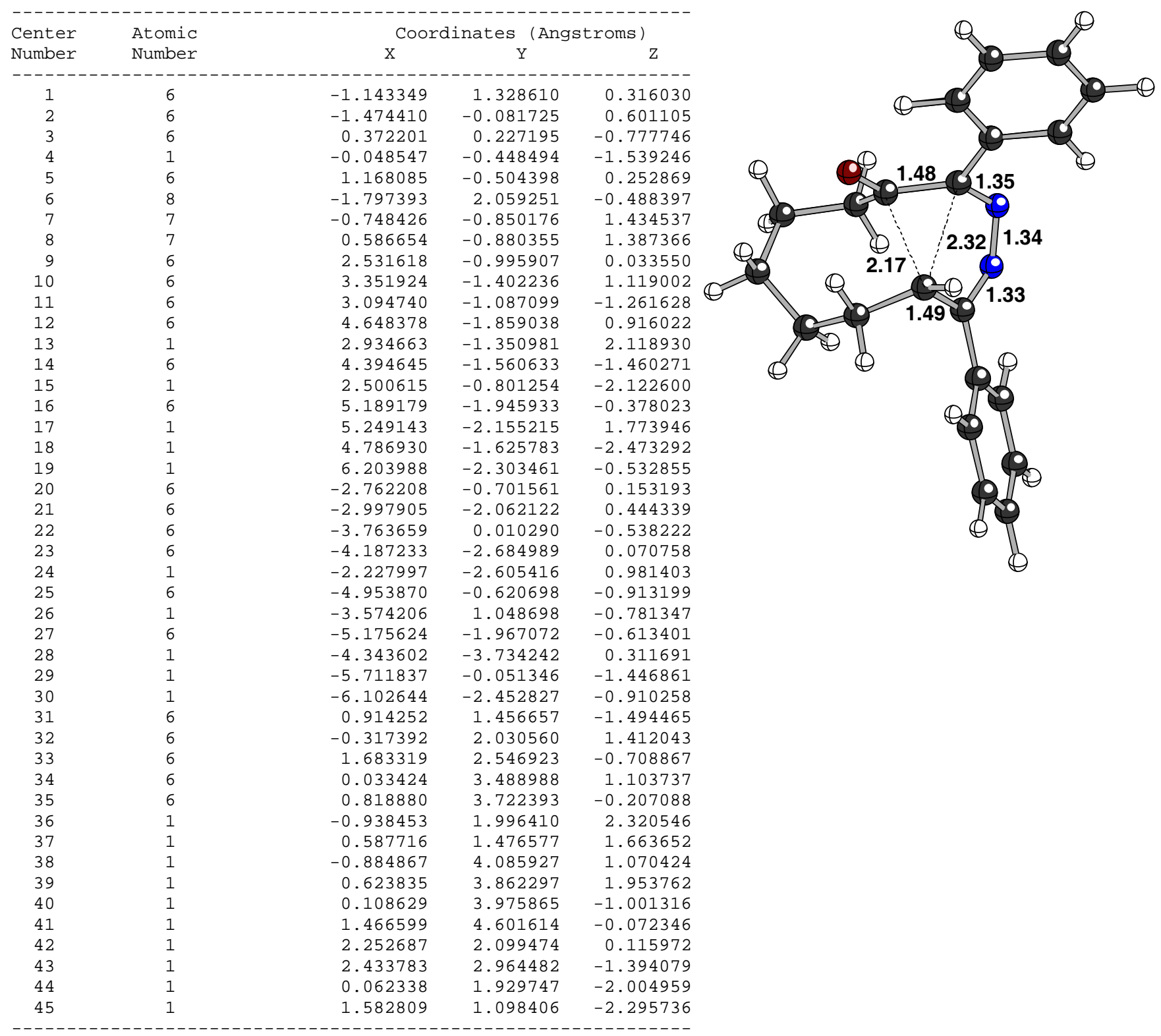




\section{1 (Figure 4)}

$\mathrm{HF}=-997.6705858$ hartrees $(-626048.269295358 \mathrm{kcal} / \mathrm{mol})$

Imaginary Frequencies: none found

Zero-point correction $=0.371512$ (Hartree/Particle)

Temperature 298.150 Kelvin. Pressure 1.00000 Atm.

Sum of electronic and thermal Free Energies $=\mathbf{- 9 9 7 . 3 4 8 1 5 5}$ hartrees $(-625845.94074405 \mathrm{kcal} / \mathrm{mol})$

Solvent Single Point: HF = -997.7515997 hartrees $(-626099.1063 \mathrm{kcal} / \mathrm{mol})$

Coordinates (from last standard orientation):

\begin{tabular}{|c|c|c|c|c|}
\hline \multirow{2}{*}{$\begin{array}{l}\text { Center } \\
\text { Number }\end{array}$} & \multirow{2}{*}{$\begin{array}{l}\text { Atomic } \\
\text { Number }\end{array}$} & \multicolumn{3}{|c|}{ Coordinates (Angstroms) } \\
\hline & & $\mathrm{x}$ & $\mathrm{Y}$ & Z \\
\hline 1 & 6 & 1.499709 & 1.470719 & $-\odot .827141$ \\
\hline 2 & 6 & 1.120195 & 0.010910 & -0.506652 \\
\hline 3 & 6 & -0.069182 & -0.148771 & 0.521462 \\
\hline 4 & 1 & 0.139215 & -1.072675 & 1.089629 \\
\hline 5 & 6 & -1.218089 & -0.430777 & -0.449173 \\
\hline 6 & 8 & 2.643670 & $1.9 \odot 4858$ & -0.743383 \\
\hline 7 & 7 & 0.545577 & -0.441672 & -1.831455 \\
\hline 8 & 7 & -0.740783 & -0.617417 & -1.687247 \\
\hline 9 & 6 & -2.583723 & -0.766696 & -0.133934 \\
\hline 10 & 6 & -3.538270 & -1.009690 & -1.166990 \\
\hline 11 & 6 & -3.062749 & -0.892921 & 1.199042 \\
\hline 12 & 6 & -4.854685 & -1.342901 & -0.881397 \\
\hline 13 & 1 & -3.202575 & -0.930253 & -2.195526 \\
\hline 14 & 6 & -4.390098 & -1.228148 & 1.475338 \\
\hline 15 & 1 & -2.383601 & -0.740382 & 2.030444 \\
\hline 16 & 6 & -5.308090 & -1.455019 & 0.445986 \\
\hline 17 & 1 & -5.546260 & -1.518413 & -1.704006 \\
\hline 18 & 1 & -4.706520 & -1.316180 & 2.513555 \\
\hline 19 & 1 & -6.341529 & -1.711444 & 0.664122 \\
\hline 20 & 6 & 2.327721 & -0.824387 & $-\odot .099149$ \\
\hline 21 & 6 & 2.595606 & -2.040739 & -0.743305 \\
\hline 22 & 6 & 3.163280 & -0.442156 & 0.964175 \\
\hline 23 & 6 & 3.656297 & -2.855309 & -0.333546 \\
\hline 24 & 1 & 1.958365 & -2.326951 & -1.573270 \\
\hline 25 & 6 & 4.221703 & -1.254388 & 1.377577 \\
\hline 26 & 1 & 2.996943 & 0.505876 & 1.463868 \\
\hline 27 & 6 & 4.475028 & -2.468245 & 0.730268 \\
\hline 28 & 1 & 3.841030 & -3.795358 & -0.849028 \\
\hline 29 & 1 & 4.854750 & -0.933922 & 2.202179 \\
\hline 30 & 1 & 5.300414 & -3.100270 & 1.050165 \\
\hline 31 & 6 & -0.235524 & 0.927316 & 1.633284 \\
\hline 32 & 6 & 0.394392 & 2.366881 & -1.380272 \\
\hline 33 & 6 & $-\odot .907774$ & 2.295437 & 1.352450 \\
\hline 34 & 6 & 0.221821 & 3.664686 & -0.574567 \\
\hline 35 & 6 & 0.033475 & 3.451992 & 0.938656 \\
\hline 36 & 1 & 0.680044 & 2.612619 & -2.410460 \\
\hline 37 & 1 & $-\odot .549553$ & 1.824207 & -1.431344 \\
\hline 38 & 1 & 1.088191 & 4.317780 & -0.735270 \\
\hline 39 & 1 & $-\odot .653278$ & 4.192353 & -0.979297 \\
\hline $4 \odot$ & 1 & 1.015296 & 3.286826 & 1.402546 \\
\hline 41 & 1 & -0.342622 & 4.392012 & 1.365989 \\
\hline 42 & 1 & -1.715584 & 2.170443 & $\odot .620535$ \\
\hline 43 & 1 & -1.398631 & 2.593771 & 2.288010 \\
\hline 44 & 1 & 0.752367 & 1.107801 & 2.081821 \\
\hline 45 & 1 & -0.815630 & ๑. 450151 & 2.430646 \\
\hline
\end{tabular}

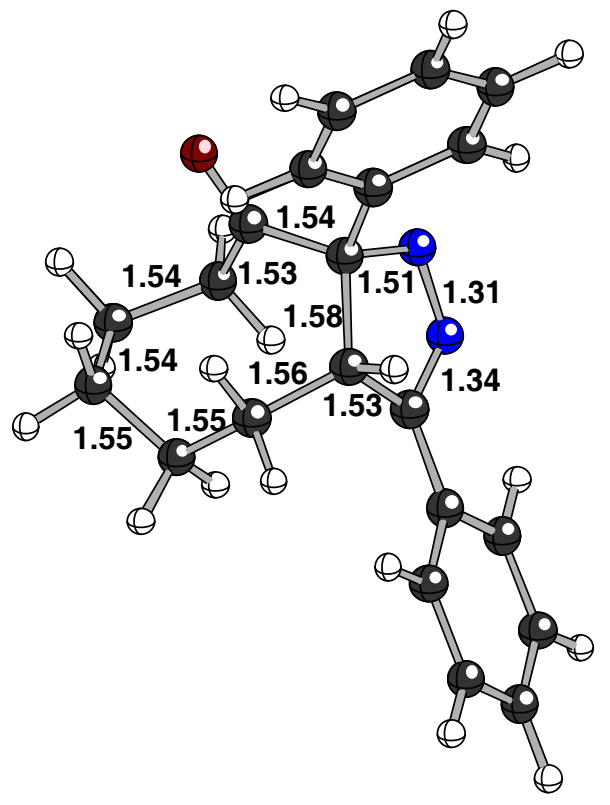




\section{Cyclopentanone reactant (Table 2 and pyridazine figure and scheme in SI)}

$\mathrm{HF}=-919.0300534$ hartrees $(-576700.548809034 \mathrm{kcal} / \mathrm{mol})$

Imaginary Frequencies: none found

Zero-point correction $=0.313824$ (Hartree/Particle)

Temperature 298.150 Kelvin. Pressure 1.00000 Atm.

Sum of electronic and thermal Free Energies = -918.762647 hartrees $(-576532.74861897 \mathbf{~ k c a l} / \mathrm{mol})$

Solvent Single Point: HF $=\mathbf{- 9 1 9 . 1 3 0 5 8 6 8 ~ h a r t r e e s ~}(-576763.6345 \mathrm{kcal} / \mathrm{mol})$

Coordinates (from last standard orientation):

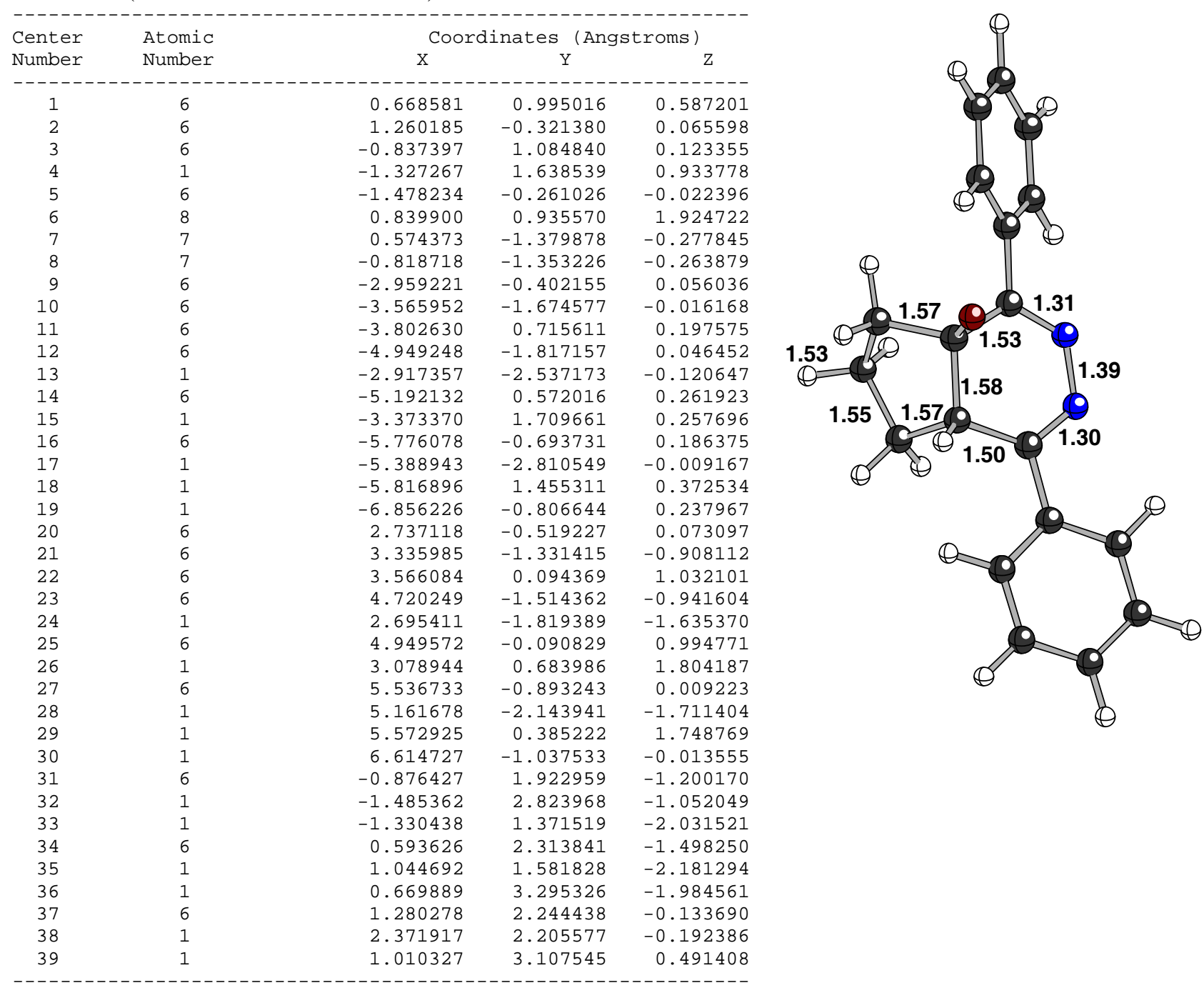




\section{Cyclopentanone reactant (Table 2 and pyridazine figure and scheme in SI)}

\section{(solvent optimization, methanol, uaks radii)}

$\mathrm{HF}=-919.133689$ hartrees $(-576765.58118439 \mathrm{kcal} / \mathrm{mol})$

Imaginary Frequencies: none found

Zero-point correction $=0.314463$ (Hartree/Particle)

Temperature 298.150 Kelvin. Pressure 1.00000 Atm.

Sum of electronic and thermal Free Energies $=\mathbf{- 9 1 8 . 8 6 5 7 3 2}$ hartrees $(-576597.43548732 \mathrm{kcal} / \mathbf{m o l})$

Coordinates (from last standard orientation):

\begin{tabular}{|c|c|c|c|c|}
\hline \multirow{2}{*}{$\begin{array}{l}\text { Center } \\
\text { Number }\end{array}$} & \multirow{2}{*}{$\begin{array}{l}\text { Atomic } \\
\text { Number }\end{array}$} & \multicolumn{3}{|c|}{ Coordinates (Angstroms) } \\
\hline & & $\mathrm{x}$ & $\mathrm{Y}$ & Z \\
\hline 1 & 6 & 0.601758 & $\odot .909710$ & 0.721826 \\
\hline 2 & 6 & 1.245809 & -0.366819 & 0.15125 \\
\hline 3 & 6 & $-\odot .808355$ & 1.037337 & 0.051393 \\
\hline 4 & 1 & -1.378033 & 1.699683 & 0.716786 \\
\hline 5 & 6 & -1.503236 & -0.286110 & -0.044631 \\
\hline 6 & 8 & $\odot .577976$ & 0.792071 & 2.095852 \\
\hline 7 & 7 & 0.558315 & -1.400476 & -0.240105 \\
\hline 8 & 7 & -0.847237 & -1.398810 & -0.179342 \\
\hline 9 & 6 & -2.988908 & -0.378340 & 0.009438 \\
\hline 10 & 6 & -3.634662 & -1.627557 & 0.118128 \\
\hline 11 & 6 & -3.789826 & 0.776760 & -0.057257 \\
\hline 12 & 6 & -5.024508 & -1.714803 & 0.158213 \\
\hline 13 & 1 & -3.029388 & -2.526210 & 0.173163 \\
\hline 14 & 6 & -5.184685 & 0.688786 & -0.023431 \\
\hline 15 & 1 & -3.330295 & 1.756231 & -0.141046 \\
\hline 16 & 6 & -5.808905 & -0.555956 & ๑.085933 \\
\hline 17 & 1 & -5.499796 & -2.688969 & 0.248461 \\
\hline 18 & 1 & -5.780727 & 1.596500 & -0.081211 \\
\hline 19 & 1 & -6.893689 & -0.625111 & 0.117088 \\
\hline 20 & 6 & 2.725472 & -0.524504 & 0.076757 \\
\hline 21 & 6 & 3.304392 & -1.194749 & $-1.01769 s$ \\
\hline 22 & 6 & 3.576183 & $-\odot .008383$ & $1.07155 \mathrm{~s}$ \\
\hline 23 & 6 & 4.689350 & -1.344446 & -1.115425 \\
\hline 24 & 1 & 2.657563 & -1.589832 & -1.795455 \\
\hline 25 & 6 & 4.960994 & -0.166035 & 0.976991 \\
\hline 26 & 1 & 3.136653 & 0.496669 & 1.925686 \\
\hline 27 & 6 & 5.524627 & -0.831638 & -0.116887 \\
\hline 28 & 1 & 5.116343 & -1.857821 & -1.974106 \\
\hline 29 & 1 & 5.601071 & 0.230454 & 1.762010 \\
\hline 30 & 1 & 6.603323 & -0.948194 & -0.191327 \\
\hline 31 & 6 & -0.596248 & 1.745908 & $-1.32749 s$ \\
\hline 32 & 1 & -1.323243 & 2.556554 & -1.446282 \\
\hline 33 & 1 & -0.748990 & 1.058509 & $-2.16721 \varepsilon$ \\
\hline 34 & 6 & 0.860192 & 2.292214 & -1.296862 \\
\hline 35 & 1 & 1.510206 & 1.661120 & -1.915436 \\
\hline 36 & 1 & 0.928438 & 3.309274 & -1.697357 \\
\hline 37 & 6 & 1.283396 & 2.197361 & 0.172636 \\
\hline 38 & 1 & 2.367111 & 2.205633 & 0.315605 \\
\hline 39 & 1 & 0.865056 & 3.031527 & 0.753386 \\
\hline
\end{tabular}

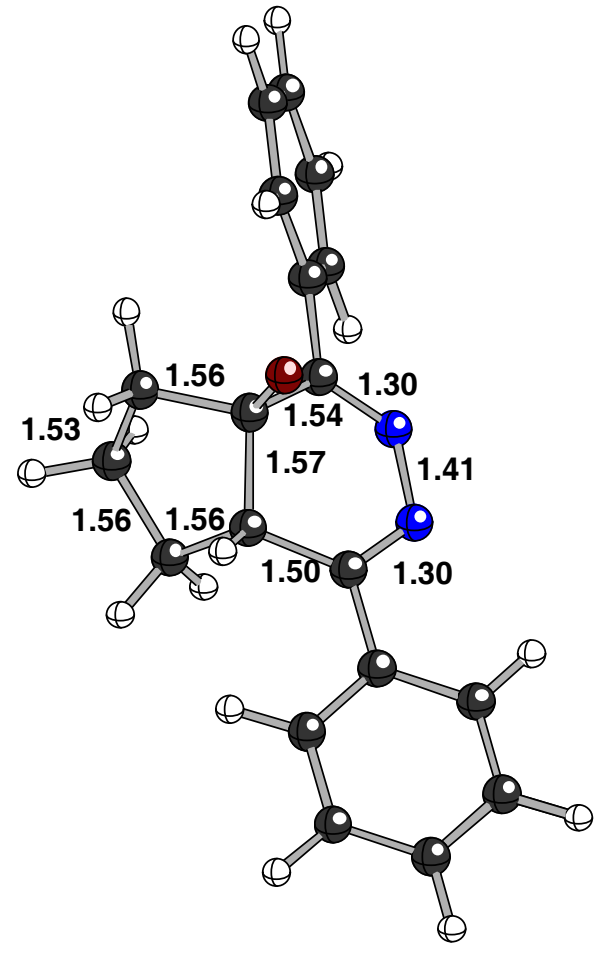




\section{Cyclopentanone reactant (Table 2 and pyridazine figure and scheme in SI)}

(solvent optimization, methanol, ua0 radii)

$\mathrm{HF}=-919.1277576$ hartrees $(-576761.859171576 \mathrm{kcal} / \mathrm{mol})$

Imaginary Frequencies: none found

Zero-point correction $=0.313472$ (Hartree/Particle)

Temperature 298.150 Kelvin. Pressure 1.00000 Atm.

Sum of electronic and thermal Free Energies $=\mathbf{- 9 1 8 . 8 6 0 2 4 7}$ hartrees $(-576593.99359497 \mathrm{kcal} / \mathrm{mol})$

Coordinates (from last standard orientation):

\begin{tabular}{|c|c|c|c|c|}
\hline \multirow{2}{*}{$\begin{array}{l}\text { Center } \\
\text { Number }\end{array}$} & \multirow{2}{*}{$\begin{array}{l}\text { Atomic } \\
\text { Number }\end{array}$} & \multicolumn{3}{|c|}{ Coordinates (Angstroms) } \\
\hline & & $x$ & $\mathrm{Y}$ & Z \\
\hline 1 & 6 & 0.596020 & $\odot .889586$ & 0.743671 \\
\hline 2 & 6 & 1.242292 & -0.364326 & 0.127717 \\
\hline 3 & 6 & -0.806452 & 1.037381 & $\odot .058968$ \\
\hline 4 & 1 & -1.383595 & 1.707342 & 0.708480 \\
\hline 5 & 6 & -1.504545 & $-\odot .280968$ & $-\odot .058280$ \\
\hline 6 & 8 & $\odot .570958$ & 0.705861 & 2.101752 \\
\hline 7 & 7 & 0.555741 & -1.390371 & -0.291049 \\
\hline 8 & 7 & $-\odot .849476$ & -1.389245 & $-\odot .232363$ \\
\hline 9 & 6 & -2.989397 & -0.375291 & ๑. 003350 \\
\hline 10 & 6 & -3.630458 & -1.621866 & 0.160348 \\
\hline 11 & 6 & -3.793949 & 0.774801 & -0.108414 \\
\hline 12 & 6 & -5.020927 & -1.712595 & 0.199939 \\
\hline 13 & 1 & -3.023484 & -2.517754 & 0.255167 \\
\hline 14 & 6 & -5.189013 & 0.682596 & $-\odot .075264$ \\
\hline 15 & 1 & -3.339432 & $1.754 \odot 42$ & -0.227720 \\
\hline 16 & 6 & -5.809241 & $-\odot .559976$ & ๑. 079481 \\
\hline 17 & 1 & -5.493890 & -2.685403 & 0.328783 \\
\hline 18 & 1 & -5.789142 & 1.586545 & -0.169857 \\
\hline 19 & 1 & -6.895597 & -0.631861 & $\odot .110251$ \\
\hline 20 & 6 & 2.722400 & -0.522642 & 0.057175 \\
\hline 21 & 6 & 3.306203 & -1.167051 & -1.050627 \\
\hline 22 & 6 & 3.570016 & -0.035938 & 1.070346 \\
\hline 23 & 6 & 4.691862 & -1.321296 & -1.142394 \\
\hline 24 & 1 & 2.663852 & -1.538473 & -1.845601 \\
\hline 25 & 6 & 4.955092 & -0.198281 & $\odot .981942$ \\
\hline 26 & 1 & 3.126069 & 0.448386 & 1.935446 \\
\hline 27 & 6 & 5.523312 & -0.839681 & -0.124782 \\
\hline 28 & 1 & 5.122766 & -1.815495 & -2.012394 \\
\hline 29 & 1 & 5.592307 & 0.174986 & 1.782799 \\
\hline 30 & 1 & 6.603449 & -0.961690 & -0.194196 \\
\hline 31 & 6 & -0.552239 & 1.727661 & -1.318160 \\
\hline 32 & 1 & -1.337714 & 2.460445 & -1.530310 \\
\hline 33 & 1 & -0.575369 & 0.997402 & -2.134567 \\
\hline 34 & 6 & $\odot .851468$ & 2.395616 & -1.206348 \\
\hline 35 & 1 & 1.559530 & 1.894858 & -1.877929 \\
\hline 36 & 1 & $\odot .826574$ & 3.450503 & -1.500937 \\
\hline 37 & 6 & 1.273313 & 2.205569 & $\odot .257062$ \\
\hline 38 & 1 & 2.357467 & 2.204453 & 0.397409 \\
\hline 39 & 1 & $\odot .860162$ & $3.0 \odot 2338$ & $\odot .890659$ \\
\hline
\end{tabular}

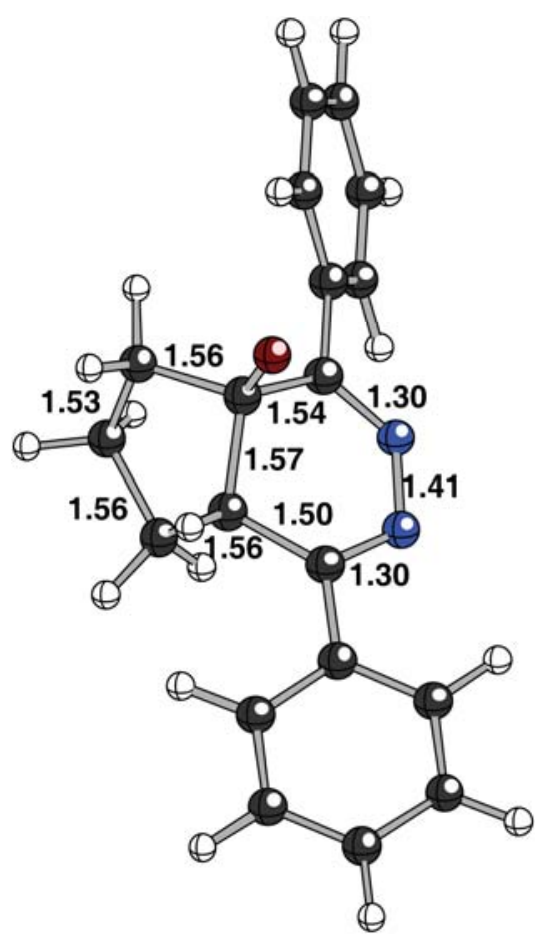




\section{Cyclopentanone Electrocyclic TS (Table 2 and pyridazine figure and scheme in SI)}

$\mathrm{HF}=-919.0097193$ hartrees $(-576687.788957943 \mathrm{kcal} / \mathrm{mol})$

Imaginary Frequencies: 1 (-267.3701 1/cm)

Zero-point correction $=0.311764$ (Hartree/Particle)

Temperature 298.150 Kelvin. Pressure 1.00000 Atm.

Sum of electronic and thermal Free Energies = $\mathbf{- 9 1 8 . 7 4 4 4 2 5}$ hartrees $(-576521.31413175 \mathrm{kcal} / \mathrm{mol})$

Solvent Single Point: HF = -919.0960133 hartrees $(-576741.9393 \mathrm{kcal} / \mathrm{mol})$

Coordinates (from last standard orientation):

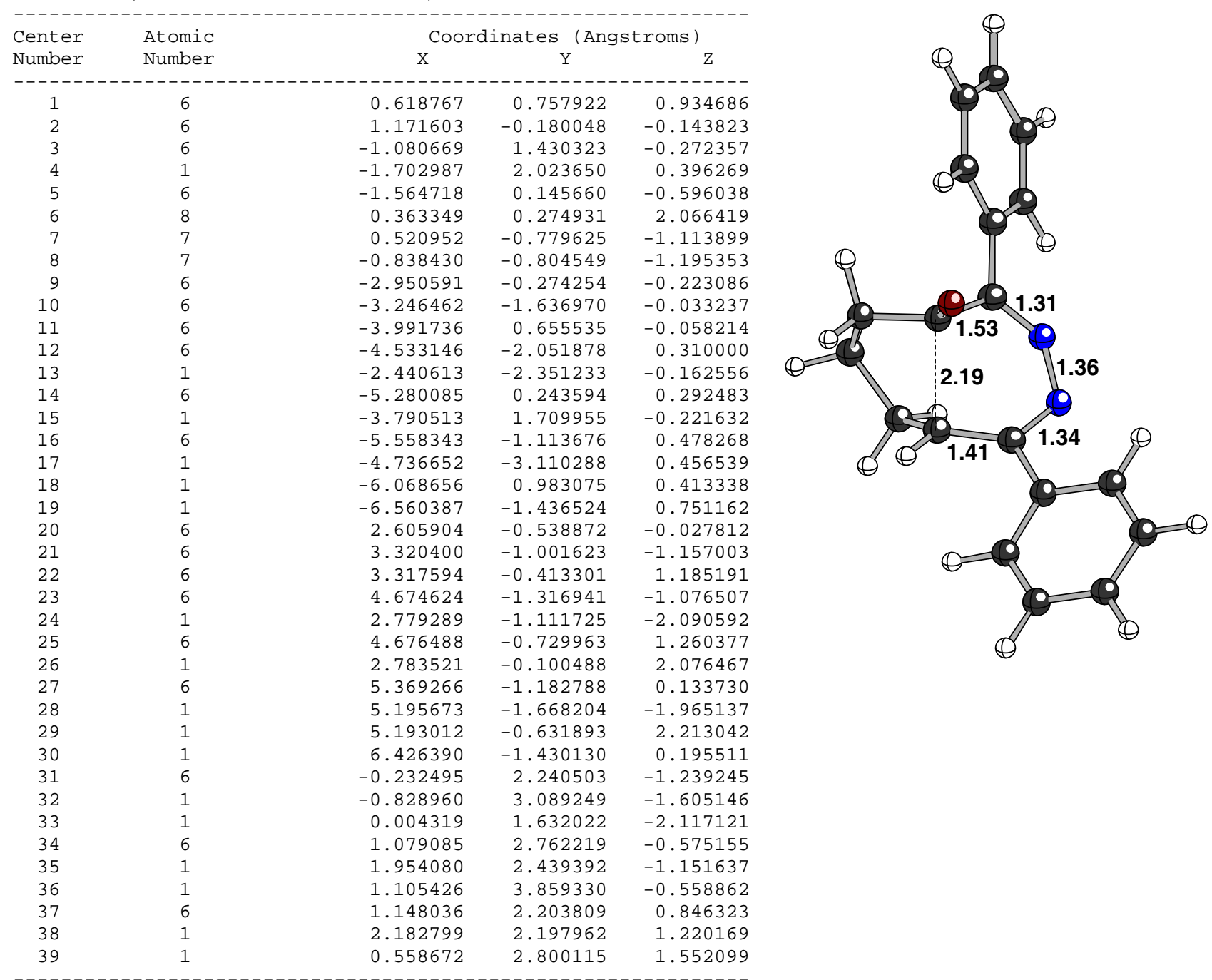




\section{Cyclopentanone Electrocyclic TS (Table 2 and pyridazine figure and scheme in SI)}

\section{(solvent optimization, methanol, uaks radii)}

$\mathrm{HF}=-919.096771$ hartrees $(-576742.41477021 \mathrm{kcal} / \mathrm{mol})$

Imaginary Frequencies: 1 (-297.4029 1/cm)

Zero-point correction $=0.311308$ (Hartree/Particle)

Temperature 298.150 Kelvin. Pressure 1.00000 Atm.

Sum of electronic and thermal Free Energies = -918.831611 hartrees $(-576576.02421861 \mathrm{kcal} / \mathrm{mol})$

Coordinates (from last standard orientation):

\begin{tabular}{|c|c|c|c|c|}
\hline \multirow{2}{*}{$\begin{array}{l}\text { Center } \\
\text { Number }\end{array}$} & \multirow{2}{*}{$\begin{array}{l}\text { Atomic } \\
\text { Number }\end{array}$} & \multicolumn{3}{|c|}{ Coordinates (Angstroms) } \\
\hline & & $\mathrm{x}$ & $\mathrm{Y}$ & Z \\
\hline----- & - - - - & -------1 & ------- & -------- \\
\hline 1 & 6 & 0.587655 & 0.627812 & 0.970972 \\
\hline 2 & 6 & 1.173661 & -0.198752 & -0.175075 \\
\hline 3 & 6 & -1.101355 & 1.444331 & -0.247406 \\
\hline 4 & 1 & -1.734902 & 2.004649 & 0.442388 \\
\hline 5 & 6 & -1.568792 & 0.183000 & -0.634418 \\
\hline 6 & 8 & 0.285093 & 0.011170 & 2.032044 \\
\hline 7 & 7 & $\odot .529990$ & -0.726388 & -1.188788 \\
\hline 8 & 7 & -0.833322 & -0.730098 & -1.309734 \\
\hline 9 & 6 & -2.947158 & -0.259515 & -0.264695 \\
\hline 10 & 6 & -3.218543 & -1.615402 & -0.003411 \\
\hline 11 & 6 & -4.008730 & ๑. 659924 & -0.172474 \\
\hline 12 & 6 & -4.503280 & -2.037549 & 0.345474 \\
\hline 13 & 1 & -2.405080 & -2.331421 & -0.070820 \\
\hline 14 & 6 & -5.295045 & ๑. 240780 & ๑. 176966 \\
\hline 15 & 1 & -3.827572 & 1.708705 & -0.392562 \\
\hline 16 & 6 & -5.547636 & -1.110358 & 0.438403 \\
\hline 17 & 1 & -4.689341 & -3.089576 & 0.550975 \\
\hline 18 & 1 & -6.102003 & ๑. 967877 & 0.236737 \\
\hline 19 & 1 & -6.548724 & -1.437769 & ๑. 710125 \\
\hline 20 & 6 & 2.619505 & -0.528292 & -0.072421 \\
\hline 21 & 6 & 3.373370 & -0.807988 & -1.234731 \\
\hline 22 & 6 & 3.299984 & -0.546810 & 1.164070 \\
\hline 23 & 6 & 4.733927 & -1.104842 & -1.161470 \\
\hline 24 & 1 & 2.874808 & -0.783446 & -2.198564 \\
\hline 25 & 6 & 4.664513 & -0.842755 & 1.234530 \\
\hline 26 & 1 & 2.749118 & -0.355115 & 2.079233 \\
\hline 27 & 6 & 5.392639 & -1.125673 & ๑. . 075017 \\
\hline 28 & 1 & 5.286203 & -1.312363 & -2.075944 \\
\hline 29 & 1 & 5.157014 & -0.858469 & 2.204678 \\
\hline 30 & 1 & 6.453984 & -1.355662 & 0.131188 \\
\hline 31 & 6 & -0.176642 & 2.297495 & -1.094750 \\
\hline 32 & 1 & -0.752076 & 3.153453 & -1.473884 \\
\hline 33 & 1 & 0.151730 & 1.734791 & -1.973587 \\
\hline 34 & 6 & 1.053710 & 2.803214 & -0.284101 \\
\hline 35 & 1 & 1.982666 & 2.623973 & -0.843157 \\
\hline 36 & 1 & ०. 994970 & 3.888181 & -0.117897 \\
\hline 37 & 6 & 1.082087 & 2.075579 & 1.063154 \\
\hline 38 & 1 & 2.104434 & 2.039705 & 1.469789 \\
\hline 39 & 1 & 0.464148 & 2.583316 & 1.812795 \\
\hline
\end{tabular}

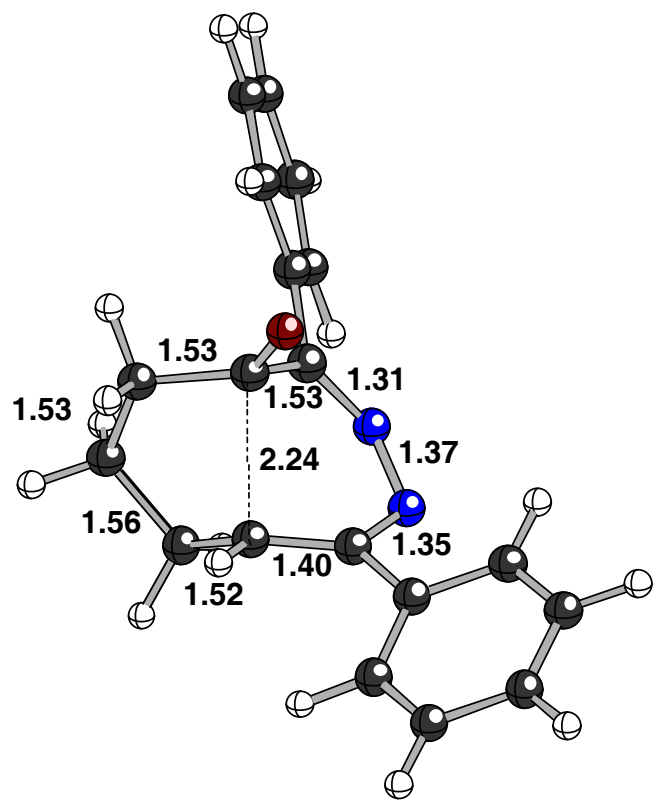




\section{Cyclopentanone Fragmentation TS (Table 2)}

$\mathrm{HF}=-918.9956259$ hartrees $(-576678.945208509 \mathrm{kcal} / \mathrm{mol})$

Imaginary Frequencies: $1(-399.95781 / \mathrm{cm})$

Zero-point correction $=0.311324$ (Hartree/Particle)

Temperature 298.150 Kelvin. Pressure 1.00000 Atm.

Sum of electronic and thermal Free Energies $=\mathbf{- 9 1 8 . 7 3 0 5 9 1}$ hartrees $(-576512.63315841 \mathrm{kcal} / \mathrm{mol})$

Solvent Single Point: HF = $\mathbf{- 9 1 9 . 0 7 9 6 6 4 5}$ hartrees $(-576731.6803 \mathrm{kcal} / \mathrm{mol})$

Coordinates (from last standard orientation):

\begin{tabular}{|c|c|c|c|c|}
\hline \multirow{2}{*}{$\begin{array}{l}\text { Center } \\
\text { Number }\end{array}$} & \multirow{2}{*}{$\begin{array}{l}\text { Atomic } \\
\text { Number }\end{array}$} & \multicolumn{3}{|c|}{ Coordinates (Angstroms) } \\
\hline & & $\mathrm{x}$ & $\mathrm{Y}$ & Z \\
\hline 1 & 6 & -1.002863 & 1.318588 & -0.166828 \\
\hline 2 & 6 & -1.405086 & -0.141538 & -0.412028 \\
\hline 3 & 6 & 1.054857 & 1.211194 & -0.847628 \\
\hline 4 & 1 & 0.536884 & 1.449603 & -1.766083 \\
\hline 5 & 6 & 1.450787 & -0.143101 & -0.681224 \\
\hline 6 & 8 & -1.561312 & 2.169413 & -0.916178 \\
\hline 7 & 7 & -0.692625 & -1.123607 & -0.900216 \\
\hline 8 & 7 & 0.676352 & -1.206561 & -0.909205 \\
\hline 9 & 6 & 2.824651 & -0.530946 & $-\odot .209697$ \\
\hline 10 & 6 & 3.010152 & -1.668783 & 0.594717 \\
\hline 11 & 6 & 3.963279 & 0.192129 & -0.604311 \\
\hline 12 & 6 & 4.286610 & -2.060913 & 1.002951 \\
\hline 13 & 1 & 2.131988 & -2.239997 & 0.877444 \\
\hline 14 & 6 & 5.242370 & -0.197979 & -0.200881 \\
\hline 15 & 1 & 3.834427 & 1.057913 & -1.247320 \\
\hline 16 & 6 & 5.410295 & -1.325543 & $\odot .60979 \odot$ \\
\hline 17 & 1 & 4.406357 & -2.942220 & 1.629259 \\
\hline 18 & 1 & 6.109106 & 0.373929 & -0.524641 \\
\hline 19 & 1 & 6.404928 & -1.629956 & 0.927282 \\
\hline 20 & 6 & -2.823573 & -0.483854 & -0.120843 \\
\hline 21 & 6 & -3.272475 & -1.822224 & $-\odot .205665$ \\
\hline 22 & 6 & -3.766493 & 0.495156 & ๑. 259617 \\
\hline 23 & 6 & -4.595867 & -2.159885 & 0.062629 \\
\hline 24 & 1 & -2.549832 & -2.578407 & -0.491520 \\
\hline 25 & 6 & -5.092820 & 0.149998 & 0.537123 \\
\hline 26 & 1 & -3.462653 & 1.534345 & 0.301015 \\
\hline 27 & 6 & -5.520796 & -1.176024 & 0.441669 \\
\hline 28 & 1 & -4.909540 & -3.199241 & -0.013063 \\
\hline 29 & 1 & -5.796356 & 0.929395 & 0.822454 \\
\hline 30 & 1 & -6.552430 & -1.441946 & 0.660533 \\
\hline 31 & 6 & 1.626361 & 2.341463 & -0.016811 \\
\hline 32 & 6 & -0.505043 & 1.804003 & 1.201770 \\
\hline 33 & 6 & 0.519019 & 2.915293 & 0.923899 \\
\hline 34 & 1 & 2.443618 & 1.965395 & ๑.608804 \\
\hline 35 & 1 & 2.045807 & 3.156960 & -0.623794 \\
\hline 36 & 1 & -0.051276 & 0.976766 & 1.764002 \\
\hline 37 & 1 & -1.352248 & 2.195635 & 1.783910 \\
\hline 38 & 1 & 0.954528 & 3.310265 & 1.851719 \\
\hline 39 & 1 & -0.009075 & 3.735229 & $\odot .425860$ \\
\hline
\end{tabular}

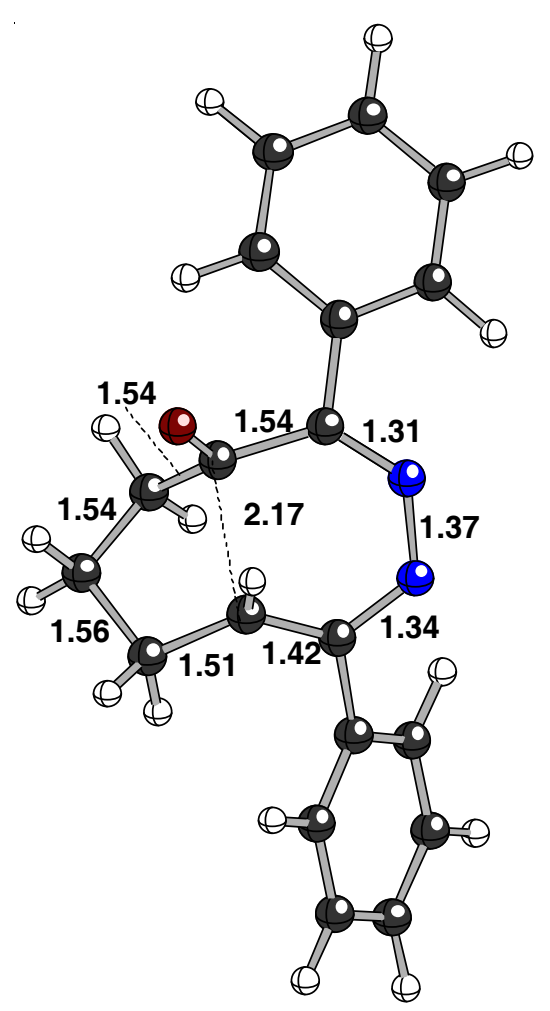




\section{Cyclopentanone Electrocyclic product (Table 2)}

$\mathrm{HF}=-919.0190476$ hartrees $(-576693.642559476 \mathrm{kcal} / \mathrm{mol})$

Imaginary Frequencies: none found

Zero-point correction $=0.311949$ (Hartree/Particle)

Temperature 298.150 Kelvin. Pressure 1.00000 Atm.

Sum of electronic and thermal Free Energies = -918.754786 hartrees $\mathbf{( - 5 7 6 5 2 7 . 8 1 5 7 6 2 8 6} \mathbf{~ k c a l} / \mathbf{m o l})$

Solvent Single Point: HF $=\mathbf{- 9 1 9 . 1 0 2 9 5 2 5}$ hartrees $(-576746.2937 \mathrm{kcal} / \mathrm{mol})$

Coordinates (from last standard orientation):

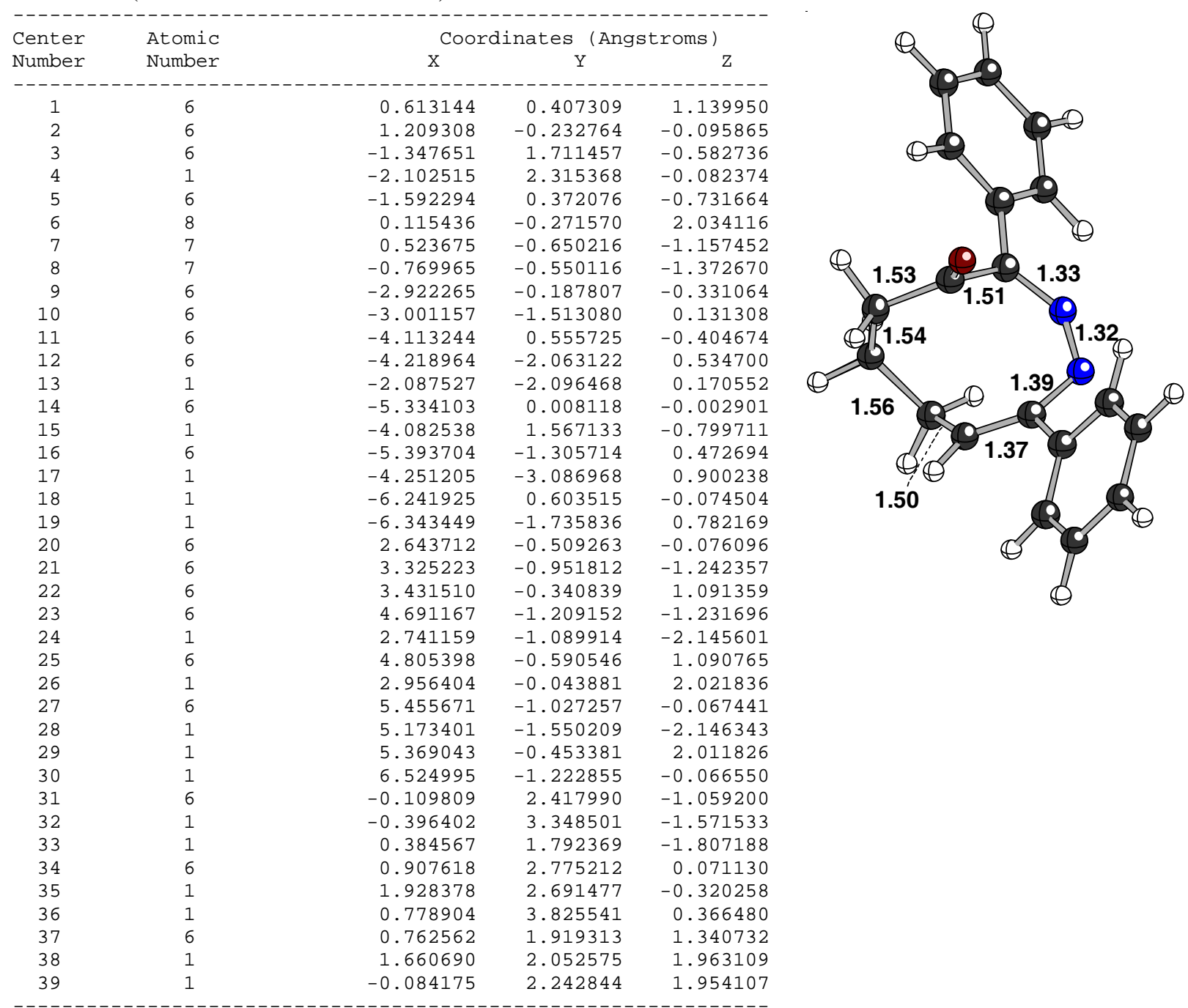




\section{Cyclopentanone Fragmentation product (Table 2)}

$\mathrm{HF}=-919.006867$ hartrees $(-576685.99911117 \mathrm{kcal} / \mathrm{mol})$

Imaginary Frequencies: none found

Zero-point correction $=0.312441$ (Hartree/Particle)

Temperature 298.150 Kelvin. Pressure 1.00000 Atm.

Sum of electronic and thermal Free Energies = -918.742027 hartrees $(-\mathbf{5 7 6 5 1 9 . 8 0 9 3 6 2 7 7} \mathbf{~ k c a l} / \mathbf{m o l})$

Solvent Single Point: HF = $\mathbf{- 9 1 9 . 0 8 9 0 4 5 ~ h a r t r e e s ~}(-576737.5666 \mathrm{kcal} / \mathrm{mol})$

Coordinates (from last standard orientation):

\begin{tabular}{|c|c|c|c|c|}
\hline \multirow{2}{*}{$\begin{array}{l}\text { Center } \\
\text { Number }\end{array}$} & \multirow{2}{*}{$\begin{array}{l}\text { Atomic } \\
\text { Number }\end{array}$} & \multicolumn{3}{|c|}{ Coordinates (Angstroms) } \\
\hline & & $\mathrm{x}$ & $\mathrm{Y}$ & Z \\
\hline 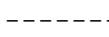 & & $--\ldots-\ldots$ & 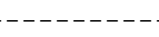 & 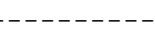 \\
\hline 1 & 6 & -1.023241 & 1.414222 & 0.238325 \\
\hline 2 & 6 & -1.404671 & 0.054606 & -0.247269 \\
\hline 3 & 6 & 1.405667 & 1.346233 & -1.302024 \\
\hline 4 & 1 & 0.533336 & 1.633783 & -1.888528 \\
\hline 5 & 6 & 1.511599 & 0.027297 & -0.989521 \\
\hline 6 & 8 & -1.749018 & 2.389481 & $-\odot .002406$ \\
\hline 7 & 7 & $-\odot .697810$ & -0.840888 & -0.965730 \\
\hline 8 & 7 & 0.545648 & -0.943935 & -1.342513 \\
\hline 9 & 6 & 2.698205 & -0.566528 & -0.302734 \\
\hline 10 & 6 & 2.518892 & -1.588201 & 0.647908 \\
\hline 11 & 6 & 4.015221 & -0.192984 & -0.626280 \\
\hline 12 & 6 & 3.611147 & -2.175786 & 1.288748 \\
\hline 13 & 1 & 1.508931 & -1.920385 & 0.866493 \\
\hline 14 & 6 & 5.110732 & -0.783093 & ๑. .008313 \\
\hline 15 & 1 & 4.172648 & 0.552936 & -1.399925 \\
\hline 16 & 6 & 4.914382 & -1.773636 & ๑. 976233 \\
\hline 17 & 1 & 3.445304 & -2.956077 & 2.027845 \\
\hline 18 & 1 & 6.119363 & -0.479697 & -0.263440 \\
\hline 19 & 1 & 5.765919 & -2.237211 & 1.468429 \\
\hline 20 & 6 & -2.829979 & -0.340823 & -0.077038 \\
\hline 21 & 6 & -3.323897 & -1.533778 & -0.663708 \\
\hline 22 & 6 & -3.749015 & ๑. 388488 & 0.716635 \\
\hline 23 & 6 & -4.634366 & -1.963435 & -0.467977 \\
\hline 24 & 1 & -2.641297 & -2.113797 & -1.273561 \\
\hline 25 & 6 & -5.060897 & -0.047302 & ๑. 912094 \\
\hline 26 & 1 & -3.439770 & 1.321296 & 1.172257 \\
\hline 27 & 6 & -5.525785 & -1.228005 & 0.324383 \\
\hline 28 & 1 & -4.964841 & -2.885769 & -0.943249 \\
\hline 29 & 1 & -5.727702 & ๑. 551116 & 1.530738 \\
\hline 30 & 1 & -6.549303 & -1.563015 & 0.475200 \\
\hline 31 & 6 & 2.040216 & 2.450605 & -0.498290 \\
\hline 32 & 6 & 0.185216 & 1.695230 & 1.150812 \\
\hline 33 & 6 & 1.053273 & 2.867445 & 0.638544 \\
\hline 34 & 1 & 2.974662 & 2.110555 & -0.043992 \\
\hline 35 & 1 & 2.279518 & 3.330763 & -1.110534 \\
\hline 36 & 1 & ๑. 780065 & $\odot .797576$ & 1.335300 \\
\hline 37 & 1 & -0.266759 & 1.990868 & 2.108899 \\
\hline 38 & 1 & 1.624498 & 3.299342 & 1.470937 \\
\hline 39 & 1 & ๑. 366520 & 3.642281 & 0.281170 \\
\hline
\end{tabular}

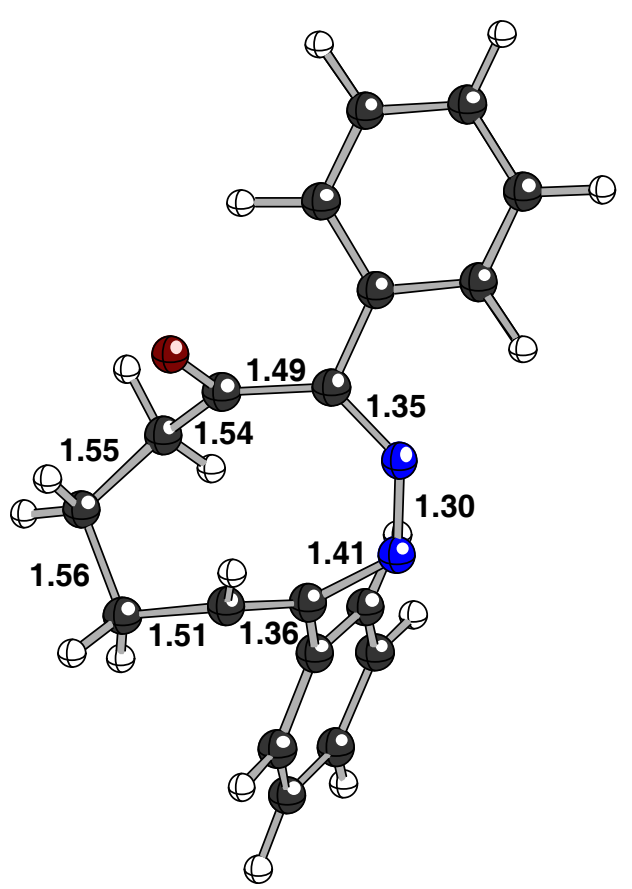




\section{Cyclohexanone reactant (Table 2)}

$\mathrm{HF}=-958.3453245$ hartrees $(-601371.274576995 \mathrm{kcal} / \mathrm{mol})$

Imaginary Frequencies: none found

Zero-point correction $=0.342536$ (Hartree/Particle)

Temperature 298.150 Kelvin. Pressure 1.00000 Atm.

Sum of electronic and thermal Free Energies = $\mathbf{- 9 5 8 . 0 4 9 7 4 4 ~ h a r t r e e s ~} \mathbf{( - 6 0 1 1 8 5 . 7 9 4 8 5 7 4 4} \mathbf{~ k c a l} / \mathrm{mol})$

Solvent Single Point: HF = -958.4389536 hartrees $(-601430.0278 \mathrm{kcal} / \mathrm{mol})$

Coordinates (from last standard orientation):

\begin{tabular}{|c|c|c|c|c|}
\hline \multirow{2}{*}{$\begin{array}{l}\text { Center } \\
\text { Number }\end{array}$} & \multirow{2}{*}{$\begin{array}{l}\text { Atomic } \\
\text { Number }\end{array}$} & \multicolumn{3}{|c|}{ Coordinates (Angstroms) } \\
\hline & & $\mathrm{x}$ & $\mathrm{Y}$ & Z \\
\hline $5--1$ & 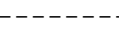 & $-\ldots$ & - & ------ \\
\hline 1 & 6 & -0.781860 & ๑. 998712 & -0.114234 \\
\hline 2 & 6 & -1.467881 & -0.397385 & -0.051891 \\
\hline 3 & 6 & 0.625090 & 0.737491 & -0.795935 \\
\hline 4 & 1 & 0.390587 & 0.552370 & -1.858097 \\
\hline 5 & 6 & 1.276139 & -0.512068 & -0.234466 \\
\hline 6 & 8 & -1.455467 & 1.938841 & -0.803898 \\
\hline 7 & 7 & -0.811444 & -1.516849 & 0.054301 \\
\hline 8 & 7 & 0.581030 & -1.508801 & 0.212685 \\
\hline 9 & 6 & 2.753680 & $-\odot .719903$ & $-\odot .184052$ \\
\hline 10 & 6 & 3.348674 & -1.317857 & ๑. 941814 \\
\hline 11 & 6 & 3.581615 & $-\odot .378599$ & -1.267861 \\
\hline 12 & 6 & 4.723052 & -1.550908 & ๑. 989055 \\
\hline 13 & 1 & 2.709905 & -1.601839 & 1.771612 \\
\hline 14 & 6 & 4.956802 & $-\odot .623250$ & -1.226918 \\
\hline 15 & 1 & 3.142523 & ๑. 059626 & -2.158618 \\
\hline 16 & 6 & 5.536818 & -1.203583 & -0.095498 \\
\hline 17 & 1 & 5.161484 & -2.006584 & 1.873804 \\
\hline 18 & 1 & 5.574775 & -0.360616 & -2.082218 \\
\hline 19 & 1 & 6.608054 & -1.385260 & -0.059504 \\
\hline 20 & 6 & -2.950478 & $-\odot .555242$ & - ๑. . 089644 \\
\hline 21 & 6 & -3.539499 & -1.702216 & ๑. 482855 \\
\hline 22 & 6 & -3.792093 & $\odot .407571$ & -0.682640 \\
\hline 23 & 6 & -4.920996 & -1.890376 & ๑. 453931 \\
\hline 24 & 1 & -2.890453 & -2.437822 & $\odot .945448$ \\
\hline 25 & 6 & -5.176732 & $\odot .212800$ & $-\odot .704937$ \\
\hline 26 & 1 & -3.302594 & 1.289268 & -1.090783 \\
\hline 27 & 6 & -5.749281 & $-\odot .932299$ & -0.143563 \\
\hline 28 & 1 & -5.353627 & -2.781823 & ๑. 903350 \\
\hline 29 & 1 & -5.812502 & ๑.964947 & -1.167561 \\
\hline 30 & 1 & -6.827691 & -1.076690 & -0.164930 \\
\hline 31 & 6 & 1.445231 & 2.040794 & -0.706298 \\
\hline 32 & 6 & -0.539109 & 1.424031 & 1.387400 \\
\hline 33 & 6 & 1.638584 & 2.530648 & 0.738488 \\
\hline 34 & 1 & 2.417995 & 1.944640 & -1.201444 \\
\hline 35 & 1 & 0.861234 & 2.787229 & -1.255922 \\
\hline 36 & 6 & 0.287035 & 2.714005 & 1. 446191 \\
\hline 37 & 1 & $-\odot .035395$ & ๑. 629129 & 1.960381 \\
\hline 38 & 1 & -1.527384 & 1.582228 & 1.835579 \\
\hline 39 & 1 & 2.198954 & 3.476949 & $\odot .731941$ \\
\hline 40 & 1 & 2.253732 & 1.810269 & 1. 299321 \\
\hline 41 & 1 & ๑. 442089 & 3.027175 & 2.489339 \\
\hline 42 & 1 & $-\odot .283795$ & 3.501431 & $\odot .939794$ \\
\hline
\end{tabular}

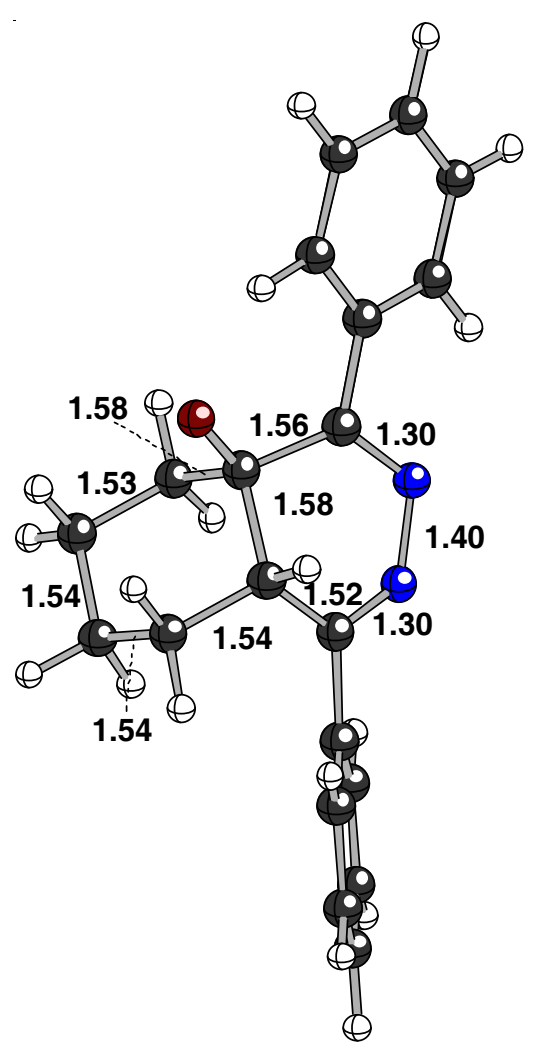




\section{Cyclohexanone 1,2-shift TS (Table 2)}

$\mathrm{HF}=-958.3065158$ hartrees $(-601346.921729658 \mathrm{kcal} / \mathrm{mol})$

Imaginary Frequencies: $1(-250.7803$ 1/cm)

Zero-point correction $=0.340117$ (Hartree/Particle)

Temperature 298.150 Kelvin. Pressure 1.00000 Atm.

Sum of electronic and thermal Free Energies $=\mathbf{- 9 5 8 . 0 1 3 6 1 9}$ hartrees $(-601163.12605869 \mathrm{kcal} / \mathrm{mol})$

Solvent Single Point: HF = -958.3835498 hartrees $(-601395.2613 \mathrm{kcal} / \mathrm{mol})$

Coordinates (from last standard orientation):

\begin{tabular}{|c|c|c|c|c|}
\hline \multirow{2}{*}{$\begin{array}{l}\text { Center } \\
\text { Number }\end{array}$} & \multirow{2}{*}{$\begin{array}{l}\text { Atomic } \\
\text { Number }\end{array}$} & \multicolumn{3}{|c|}{ Coordinates (Angstroms) } \\
\hline & & $\mathrm{x}$ & $\mathrm{Y}$ & Z \\
\hline 1 & 6 & 1.211354 & 1.545909 & -0.260400 \\
\hline 2 & 6 & 1.454594 & 0.130297 & -0.584054 \\
\hline 3 & 6 & -0.457630 & 0.531378 & 0.788912 \\
\hline 4 & 1 & -0.144864 & -0.077422 & 1.650833 \\
\hline 5 & 6 & -1.213098 & -0.221445 & -0.240933 \\
\hline 6 & 8 & 1.889021 & 2.211014 & 0.573522 \\
\hline 7 & 7 & 0.695021 & -0.570463 & -1.448728 \\
\hline 8 & 7 & -0.633753 & -0.576535 & -1.390886 \\
\hline 9 & 6 & -2.555638 & -0.759942 & -0.007430 \\
\hline 10 & 6 & -3.326800 & -1.320305 & -1.060921 \\
\hline 11 & 6 & -3.151515 & -0.737735 & 1.276868 \\
\hline 12 & 6 & -4.607778 & -1.810279 & -0.839194 \\
\hline 13 & 1 & -2.882837 & -1.360481 & -2.049541 \\
\hline 14 & 6 & -4.432271 & -1.252620 & 1.496915 \\
\hline 15 & 1 & -2.598413 & -0.324358 & 2.113301 \\
\hline 16 & 6 & -5.178909 & -1.787491 & 0.444759 \\
\hline 17 & 1 & -5.170840 & -2.224733 & -1.673492 \\
\hline 18 & 1 & -4.849138 & -1.227829 & 2.501917 \\
\hline 19 & 1 & -6.179906 & -2.176050 & 0.614358 \\
\hline 20 & 6 & 2.702831 & -0.573624 & -0.152149 \\
\hline 21 & 6 & 2.856702 & -1.940095 & -0.471257 \\
\hline 22 & 6 & 3.744897 & 0.060057 & $\odot .555773$ \\
\hline 23 & 6 & 4.006914 & -2.640888 & $-\odot .113507$ \\
\hline 24 & 1 & 2.055690 & -2.426342 & -1.017244 \\
\hline 25 & 6 & 4.894865 & -0.649121 & 0.915630 \\
\hline 26 & 1 & 3.619317 & 1.101401 & 0.824900 \\
\hline 27 & 6 & 5.036589 & -1.999243 & 0.585199 \\
\hline 28 & 1 & 4.099121 & -3.692409 & -0.376665 \\
\hline 29 & 1 & 5.684664 & -0.138168 & 1.462128 \\
\hline 30 & 1 & 5.932454 & -2.546275 & 0.870871 \\
\hline 31 & 6 & -0.970986 & 1.875769 & 1.293182 \\
\hline 32 & 6 & 0.417877 & 2.358282 & -1.305606 \\
\hline 33 & 6 & -1.526028 & 2.854535 & 0.244834 \\
\hline 34 & 1 & -1.774160 & 1.677717 & 2.024864 \\
\hline 35 & 1 & -0.157781 & 2.363839 & 1.850208 \\
\hline 36 & 6 & -0.435241 & 3.448595 & -0.654819 \\
\hline 37 & 1 & $-\odot .191950$ & 1.723808 & -1.950856 \\
\hline 38 & 1 & 1.189987 & 2.819536 & -1.939063 \\
\hline 39 & 1 & -2.048411 & 3.667559 & $\odot .768871$ \\
\hline 40 & 1 & -2.278638 & 2.340987 & $-\odot .368807$ \\
\hline 41 & 1 & -0.891331 & 4.077115 & -1.432663 \\
\hline 42 & 1 & 0.221635 & 4.090778 & $-\odot .055608$ \\
\hline
\end{tabular}

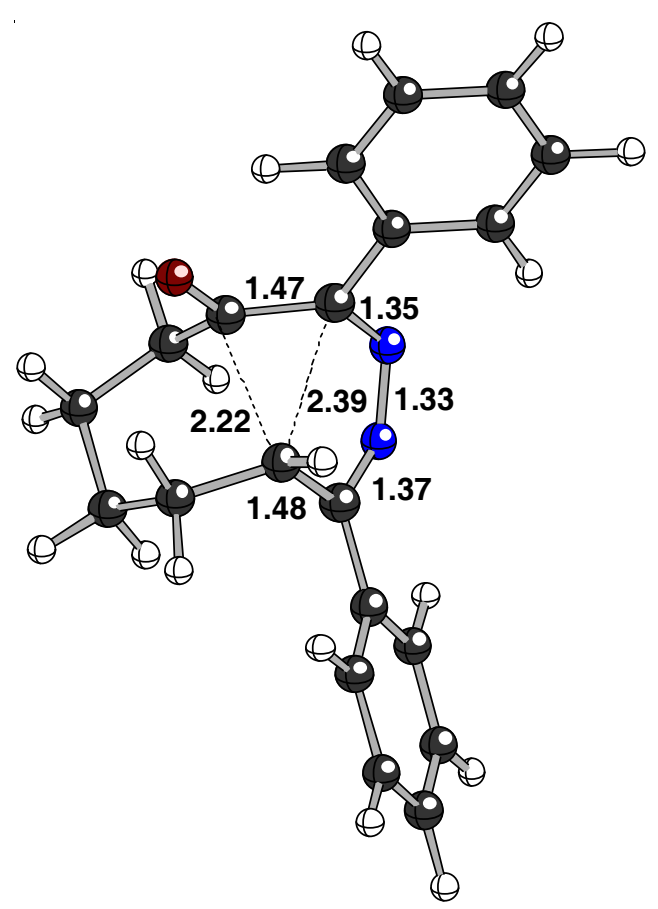




\section{Cyclohexanone Fragmentation TS (Table 2)}

$\mathrm{HF}=-958.3179839$ hartrees $(-601354.118077089 \mathrm{kcal} / \mathrm{mol})$

Imaginary Frequencies: 1 (-374.4852 1/cm)

Zero-point correction $=0.340125$ (Hartree/Particle)

Temperature 298.150 Kelvin. Pressure 1.00000 Atm.

Sum of electronic and thermal Free Energies = -958.025301 hartrees $\mathbf{( - 6 0 1 1 7 0 . 4 5 6 6 3 0 5 1 ~} \mathbf{k c a l} / \mathrm{mol})$

Solvent Single Point: HF $=\mathbf{- 9 5 8 . 3 9 8 7 7 2 3}$ hartrees $(-601404.8136 \mathrm{kcal} / \mathrm{mol})$

Coordinates (from last standard orientation):

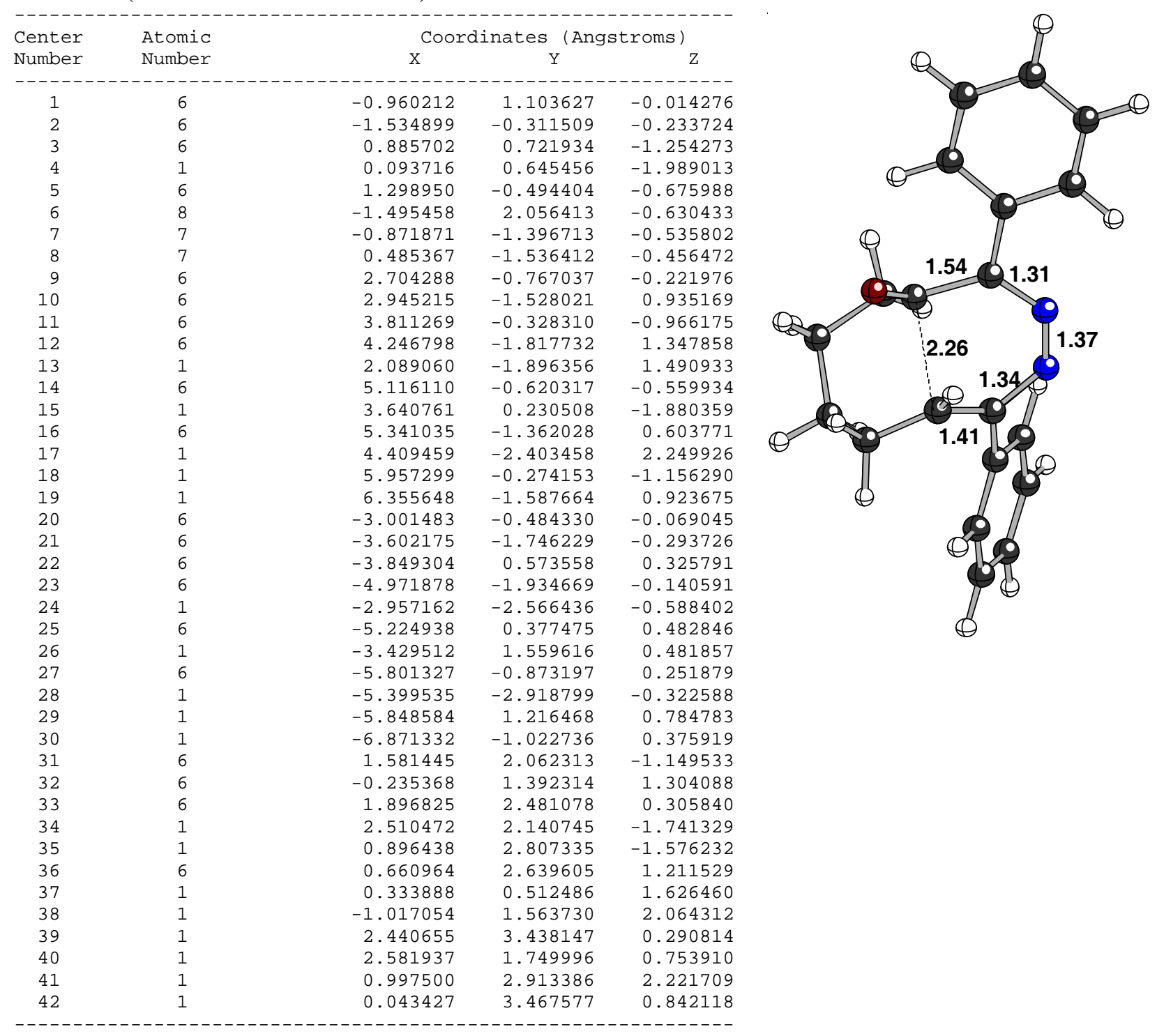




\section{Cyclohexanone 1,2-shift product (Table 2)}

$\mathrm{HF}=-958.3657059$ hartrees $(-601384.064109309 \mathrm{kcal} / \mathrm{mol})$

Imaginary Frequencies: none found

Zero-point correction $=0.342883$ (Hartree/Particle)

Temperature 298.150 Kelvin. Pressure 1.00000 Atm.

Sum of electronic and thermal Free Energies $=\mathbf{- 9 5 8 . 0 7 0 6 2 7}$ hartrees $(-601198.89914877 \mathrm{kcal} / \mathrm{mol})$

Solvent Single Point: HF = -958.4476732 hartrees $(-601435.4994 \mathrm{kcal} / \mathrm{mol})$

Coordinates (from last standard orientation):

\begin{tabular}{|c|c|c|c|c|}
\hline \multirow{2}{*}{$\begin{array}{l}\text { Center } \\
\text { Number }\end{array}$} & \multirow{2}{*}{$\begin{array}{l}\text { Atomic } \\
\text { Number }\end{array}$} & \multicolumn{3}{|c|}{ Coordinates (Angstroms) } \\
\hline & & $\mathrm{x}$ & $\mathrm{Y}$ & Z \\
\hline 1 & 6 & 1.555119 & 1.732306 & -0.669455 \\
\hline 2 & 6 & 1.138950 & 0.263346 & -0.462991 \\
\hline 3 & 6 & -0.060557 & 0.074242 & 0.544634 \\
\hline 4 & 1 & 0.148934 & -0.830280 & 1.139958 \\
\hline 5 & 6 & -1.187535 & -0.215623 & -0.446821 \\
\hline 6 & 8 & 2.723593 & 2.103710 & -0.637916 \\
\hline 7 & 7 & 0.574882 & -0.084195 & -1.822272 \\
\hline 8 & 7 & -0.707721 & -0.296625 & -1.694754 \\
\hline 9 & 6 & -2.539782 & -0.607865 & -0.145455 \\
\hline 10 & 6 & -3.494500 & -0.835797 & -1.181212 \\
\hline 11 & 6 & -3.001538 & -0.806218 & 1.185040 \\
\hline 12 & 6 & -4.797846 & -1.220438 & -0.899497 \\
\hline 13 & 1 & -3.170249 & -0.701843 & -2.207851 \\
\hline 14 & 6 & -4.314914 & -1.194546 & 1.457027 \\
\hline 15 & 1 & -2.317314 & -0.668648 & 2.015779 \\
\hline 16 & 6 & -5.234974 & -1.404000 & 0.425375 \\
\hline 17 & 1 & -5.491771 & -1.381264 & -1.723122 \\
\hline 18 & 1 & -4.619354 & -1.337766 & 2.492655 \\
\hline 19 & 1 & -6.258224 & -1.700897 & 0.640181 \\
\hline 20 & 6 & 2.324254 & -0.624510 & -0.101102 \\
\hline 21 & 6 & 2.591467 & -1.788326 & -0.834371 \\
\hline 22 & 6 & 3.136772 & -0.341120 & 1.009576 \\
\hline 23 & 6 & 3.631881 & -2.648308 & $-\odot .466140$ \\
\hline 24 & 1 & 1.970065 & -1.997275 & -1.698730 \\
\hline 25 & 6 & 4.174751 & -1.197702 & 1.381443 \\
\hline 26 & 1 & 2.966718 & 0.565026 & 1.582278 \\
\hline 27 & 6 & 4.428983 & -2.359258 & 0.643995 \\
\hline 28 & 1 & 3.817084 & -3.547228 & -1.050226 \\
\hline 29 & 1 & 4.791156 & -0.953774 & 2.243976 \\
\hline 30 & 1 & 5.238288 & -3.026713 & 0.931319 \\
\hline 31 & 6 & -0.278946 & 1.200008 & 1.596571 \\
\hline 32 & 6 & 0.457335 & 2.740661 & -0.995972 \\
\hline 33 & 6 & -0.968021 & 2.492897 & 1.127159 \\
\hline 34 & 1 & $-\odot .880355$ & 0.781528 & 2.411915 \\
\hline 35 & 1 & 0.692018 & 1.447460 & 2.052061 \\
\hline 36 & 6 & -0.092752 & 3.418759 & 0.274660 \\
\hline 37 & 1 & -0.358876 & 2.257440 & -1.538706 \\
\hline 38 & 1 & ๑.916496 & 3.492594 & -1.646713 \\
\hline 39 & 1 & -1.306463 & 3.048305 & 2.013568 \\
\hline $4 \odot$ & 1 & -1.869258 & 2.223641 & 0.562038 \\
\hline 41 & 1 & -0.684267 & 4.294877 & -0.025420 \\
\hline 42 & 1 & 0.747630 & 3.799814 & $\odot .872763$ \\
\hline
\end{tabular}

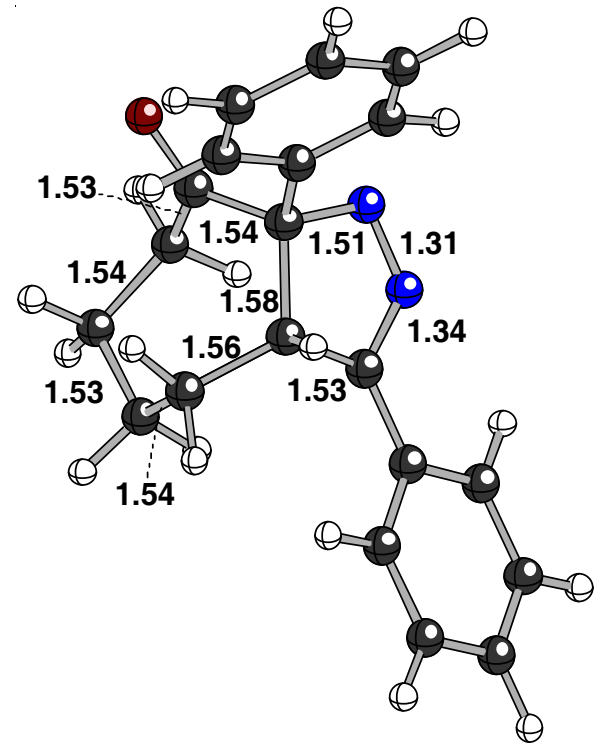




\section{Cyclohexanone Fragmentation product (Table 2)}

$\mathrm{HF}=-958.3256389$ hartrees $(-601358.921666139 \mathrm{kcal} / \mathrm{mol})$

Imaginary Frequencies: none found

Zero-point correction $=0.340904$ (Hartree/Particle)

Temperature 298.150 Kelvin. Pressure 1.00000 Atm.

Sum of electronic and thermal Free Energies = -958.033665 hartrees $(-601175.70512415 \mathrm{kcal} / \mathrm{mol})$

Solvent Single Point: HF = -958.40535 hartrees $(-\mathbf{6 0 1 4 0 8 . 9 4 1 2 ~ k c a l} / \mathrm{mol})$

Coordinates (from last standard orientation):

\begin{tabular}{|c|c|c|c|c|}
\hline \multirow{2}{*}{$\begin{array}{l}\text { Center } \\
\text { Number }\end{array}$} & \multirow{2}{*}{$\begin{array}{l}\text { Atomic } \\
\text { Number }\end{array}$} & \multicolumn{3}{|c|}{ Coordinates (Angstroms) } \\
\hline & & $\mathrm{X}$ & $\mathrm{Y}$ & Z \\
\hline \multicolumn{5}{|c|}{ 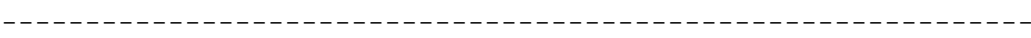 } \\
\hline 1 & 6 & -1.137780 & 1.242816 & ๑. 220818 \\
\hline 2 & 6 & -1.602738 & -0.130254 & -0.135051 \\
\hline 3 & 6 & 1.162682 & 0.864388 & -1.432008 \\
\hline 4 & 1 & 0.183977 & 1.014567 & -1.877754 \\
\hline 5 & 6 & 1.359377 & -0.344353 & -0.831373 \\
\hline 6 & 8 & -1.708811 & 2.252140 & -0.206010 \\
\hline 7 & 7 & -0.871974 & -1.184398 & -0.534249 \\
\hline 8 & 7 & 0.402131 & -1.372239 & -0.757560 \\
\hline 9 & 6 & 2.678851 & $-\odot .784319$ & - ๑. 278191 \\
\hline 10 & 6 & 2.723480 & -1.570487 & 0.887459 \\
\hline 11 & 6 & 3.895654 & -0.494219 & -0.919460 \\
\hline 12 & 6 & 3.938945 & -2.003336 & 1.421077 \\
\hline 13 & 1 & 1.785374 & -1.849189 & 1.356736 \\
\hline 14 & 6 & 5.113770 & -0.930027 & -0.392983 \\
\hline 15 & 1 & 3.879371 & ๑. 060730 & -1.851936 \\
\hline 16 & 6 & 5.143306 & -1.680521 & ๑. 787096 \\
\hline 17 & 1 & 3.945748 & -2.602880 & 2.328490 \\
\hline 18 & 1 & 6.040442 & $-\odot .694999$ & -0.911875 \\
\hline 19 & 1 & 6.090399 & -2.022579 & 1.197458 \\
\hline 20 & 6 & -3.058790 & -0.409635 & -0.053308 \\
\hline 21 & 6 & -3.560432 & -1.696530 & -0.380108 \\
\hline 22 & 6 & -4.014492 & 0.541355 & ๑. 382108 \\
\hline 23 & 6 & -4.912488 & -2.008154 & -0.269004 \\
\hline 24 & 1 & -2.850064 & -2.441324 & -0.719242 \\
\hline 25 & 6 & -5.370131 & ๑. 222703 & ๑. 489765 \\
\hline 26 & 1 & -3.695926 & 1.549068 & 0.618202 \\
\hline 27 & 6 & -5.841498 & -1.053477 & 0.168023 \\
\hline 28 & 1 & -5.247387 & -3.010540 & -0.531237 \\
\hline 29 & 1 & -6.065414 & ๑. 990052 & ๑. 826042 \\
\hline 30 & 1 & -6.898394 & -1.296410 & ๑. 248090 \\
\hline 31 & 6 & 1.976159 & 2.128549 & -1.236947 \\
\hline 32 & 6 & -0.039713 & 1.441475 & 1.274524 \\
\hline 33 & 6 & 2.139997 & 2.515336 & ๑. 253868 \\
\hline 34 & 1 & 2.979988 & 2.081231 & -1.683302 \\
\hline 35 & 1 & 1.460691 & 2.946343 & -1.757270 \\
\hline 36 & 6 & ๑. 839899 & 2.696027 & 1.064682 \\
\hline 37 & 1 & 0.554283 & 0.535228 & 1.409431 \\
\hline 38 & 1 & -0.615367 & 1.571640 & 2.206069 \\
\hline 39 & 1 & 2.706857 & 3.458342 & 0.307560 \\
\hline 40 & 1 & 2.762599 & 1.757653 & 0.745662 \\
\hline 41 & 1 & 1.127754 & 3.082732 & 2.052084 \\
\hline 42 & 1 & ๑. 210104 & 3.465645 & ๑. 601627 \\
\hline
\end{tabular}

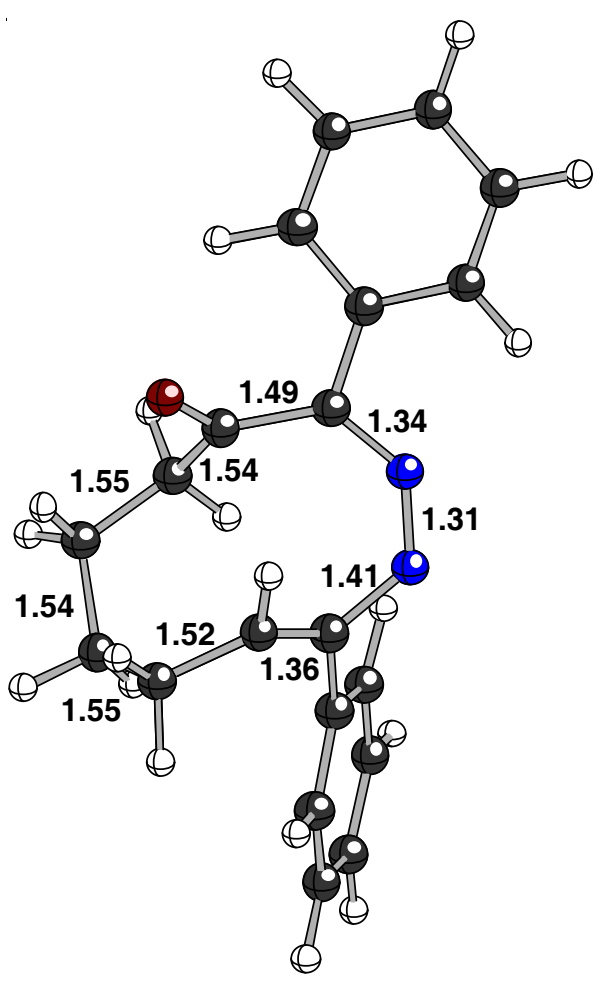




\section{2,2,6-Trimethylcyclohexanone reactant (Table 2)}

$\mathrm{HF}=-1076.2851767$ hartrees $(-675379.711231017 \mathrm{kcal} / \mathrm{mol})$

Imaginary Frequencies: none found

Zero-point correction $=0.426087$ (Hartree/Particle)

Temperature 298.150 Kelvin. Pressure 1.00000 Atm.

Sum of electronic and thermal Free Energies $=\mathbf{- 1 0 7 5 . 9 0 8 7 1 9}$ hartrees $(-675143.48025969 \mathrm{kcal} / \mathrm{mol})$

Solvent Single Point: HF = -1076.367877 hartrees $(-675431.6062 \mathrm{kcal} / \mathrm{mol})$

Coordinates (from last standard orientation):

\begin{tabular}{|c|c|c|c|c|}
\hline \multirow{2}{*}{$\begin{array}{l}\text { Center } \\
\text { Number }\end{array}$} & \multirow{2}{*}{$\begin{array}{l}\text { Atomic } \\
\text { Number }\end{array}$} & \multicolumn{3}{|c|}{ Coordinates (Angstroms) } \\
\hline & & $\mathrm{x}$ & $\mathrm{Y}$ & Z \\
\hline 1 & 6 & 0.720424 & 0.836444 & 0.340995 \\
\hline 2 & 6 & 1.389906 & $-\odot .557569$ & 0.080496 \\
\hline 3 & 6 & -0.697440 & 0.463893 & 1.038091 \\
\hline 4 & 6 & -1.362390 & -0.670187 & 0.268045 \\
\hline 5 & 8 & 1.433560 & 1.601587 & 1.183048 \\
\hline 6 & 7 & 0.718721 & -1.655662 & -0.108959 \\
\hline 7 & 7 & -0.667824 & -1.609981 & -0.288064 \\
\hline 8 & 6 & -2.840327 & -0.827761 & $\odot .092579$ \\
\hline 9 & 6 & -3.350865 & -1.254490 & -1.148060 \\
\hline 10 & 6 & -3.753359 & -0.617501 & 1.140962 \\
\hline 11 & 6 & -4.719494 & -1.444077 & -1.338847 \\
\hline 12 & 1 & -2.650934 & -1.443548 & -1.954817 \\
\hline 13 & 6 & -5.123922 & -0.817646 & 0.954713 \\
\hline 14 & 1 & -3.389320 & -0.322101 & 2.118172 \\
\hline 15 & 6 & -5.616571 & -1.224602 & -0.287815 \\
\hline 16 & 1 & -5.087109 & -1.765613 & -2.310449 \\
\hline 17 & 1 & -5.806278 & -0.658144 & 1.786149 \\
\hline 18 & 1 & -6.683579 & -1.372264 & -0.434623 \\
\hline 19 & 6 & 2.869548 & -0.758974 & 0.103359 \\
\hline 20 & 6 & 3.425466 & -1.804813 & -0.662729 \\
\hline 21 & 6 & 3.740215 & 0.044203 & 0.867177 \\
\hline 22 & 6 & 4.799877 & -2.044463 & -0.666172 \\
\hline 23 & 1 & 2.757499 & -2.423348 & -1.252315 \\
\hline 24 & 6 & 5.116275 & -0.200146 & 0.857878 \\
\hline 25 & 1 & 3.285127 & 0.851733 & 1.433224 \\
\hline 26 & 6 & 5.655616 & -1.242003 & 0.096662 \\
\hline 27 & 1 & 5.203944 & -2.854735 & -1.269420 \\
\hline 28 & 1 & 5.772330 & 0.430532 & 1.454259 \\
\hline 29 & 1 & 6.728216 & -1.424887 & $\odot .093332$ \\
\hline 30 & 6 & -1.510948 & 1.771525 & 1.153043 \\
\hline 31 & 6 & 0.513334 & 1.604630 & -1.080732 \\
\hline 32 & 6 & -1.711301 & 2.520316 & -0.168474 \\
\hline 33 & 1 & -2.477872 & 1.593148 & 1.636987 \\
\hline 34 & 1 & -0.925638 & 2.412912 & 1.822930 \\
\hline 35 & 6 & -0.358758 & 2.850801 & -0.807002 \\
\hline 36 & 1 & -2.268856 & 3.448708 & ๑. 023198 \\
\hline 37 & 1 & -2.333338 & 1.930004 & -0.854958 \\
\hline 38 & 1 & -0.504937 & 3.401715 & -1.749687 \\
\hline 39 & 1 & 0.199442 & 3.504383 & -0.125996 \\
\hline $4 \odot$ & 6 & -0.410400 & -0.044370 & 2.476044 \\
\hline 41 & 1 & 0.075016 & -1.025716 & 2.473576 \\
\hline 42 & 1 & 0.264061 & 0.674748 & 2.949105 \\
\hline 43 & 1 & -1.337986 & -0.125300 & 3.057449 \\
\hline 44 & 6 & 1.896973 & 2.091886 & -1.553006 \\
\hline 45 & 1 & 2.404359 & 2.605947 & -0.733255 \\
\hline 46 & 1 & 2.530484 & 1.259366 & -1.880246 \\
\hline 47 & 1 & 1.783627 & 2.778149 & -2.404991 \\
\hline 48 & 6 & -0.104489 & 0.790687 & -2.240059 \\
\hline 49 & 1 & 0.445532 & -0.137733 & -2.425105 \\
\hline 50 & 1 & -1.151152 & 0.525477 & -2.078252 \\
\hline 51 & 1 & -0.061634 & 1.388669 & -3.162378 \\
\hline
\end{tabular}

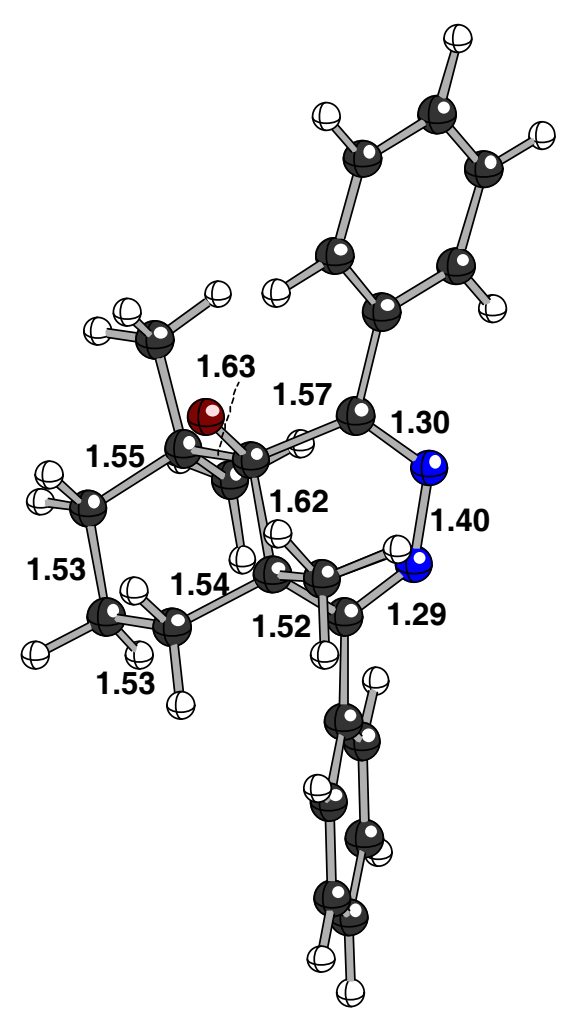




\section{2,2,6-Trimethylcyclohexanone 1,2-shift TS (Table 2)}

$\mathrm{HF}=-1076.245302$ hartrees $(-675354.68945802 \mathrm{kcal} / \mathrm{mol})$ Imaginary Frequencies: 1 (-160.3784 1/cm)

Zero-point correction $=0.423385$ (Hartree/Particle)

Temperature 298.150 Kelvin. Pressure 1.00000 Atm.

Sum of electronic and thermal Free Energies = -1075.873117 hartrees $(-675121.13964867 \mathrm{kcal} / \mathrm{mol})$

Solvent Single Point: HF = -1076.31591 hartrees $(-675398.9964 \mathrm{kcal} / \mathrm{mol})$

Coordinates (from last standard orientation):

\begin{tabular}{|c|c|c|c|c|}
\hline \multirow{2}{*}{$\begin{array}{l}\text { Center } \\
\text { Number }\end{array}$} & \multirow{2}{*}{$\begin{array}{l}\text { Atomic } \\
\text { Number }\end{array}$} & \multicolumn{3}{|c|}{ Coordinates (Angstroms) } \\
\hline & & $\mathrm{x}$ & $\mathrm{Y}$ & Z \\
\hline$x^{2}+2=$ & & --------1 & ------ & ------- \\
\hline 1 & 6 & 1.266653 & 1.219195 & 0.192528 \\
\hline 2 & 6 & 1.368120 & -0.106889 & -0.443974 \\
\hline 3 & 6 & -0.652396 & 0.148097 & 1.012320 \\
\hline 4 & 6 & -1.379971 & -0.354399 & -0.173702 \\
\hline 5 & 8 & 1.890124 & 1.500278 & 1.253918 \\
\hline 6 & 7 & $\odot .552498$ & -0.578281 & -1.400546 \\
\hline 7 & 7 & -0.769547 & -0.506161 & -1.353805 \\
\hline 8 & 6 & -2.777572 & -0.806097 & -0.132897 \\
\hline 9 & 6 & -3.483156 & -1.139085 & -1.320640 \\
\hline 10 & 6 & -3.501391 & $-\odot .898798$ & 1.080477 \\
\hline 11 & 6 & -4.817834 & -1.525261 & -1.288480 \\
\hline 12 & 1 & -2.946293 & -1.086997 & -2.261159 \\
\hline 13 & 6 & -4.838085 & -1.304276 & 1.107610 \\
\hline 14 & 1 & -3.009688 & $-\odot .661262$ & 2.016254 \\
\hline 15 & 6 & -5.515456 & -1.618604 & -0.073039 \\
\hline 16 & 1 & -5.323510 & -1.767378 & -2.221542 \\
\hline 17 & 1 & -5.352409 & -1.370555 & 2.064368 \\
\hline 18 & 1 & -6.556578 & -1.930505 & -0.051789 \\
\hline 19 & 6 & 2.556655 & -0.985495 & -0.185603 \\
\hline 20 & 6 & 2.607453 & -2.255551 & -0.802467 \\
\hline 21 & 6 & 3.645341 & -0.613097 & 0.630771 \\
\hline 22 & 6 & 3.697560 & -3.105549 & -0.627042 \\
\hline 23 & 1 & 1.773600 & -2.545718 & -1.431968 \\
\hline 24 & 6 & 4.735558 & -1.470643 & ๑. 805958 \\
\hline 25 & 1 & 3.607682 & ๑. 343151 & 1.136025 \\
\hline 26 & 6 & 4.772889 & -2.719936 & ๑.181983 \\
\hline 27 & 1 & 3.705991 & -4.075414 & -1.119780 \\
\hline 28 & 1 & 5.559704 & -1.156679 & 1.442925 \\
\hline 29 & 1 & 5.620925 & -3.385417 & $\odot .327434$ \\
\hline 30 & 6 & -1.069556 & 1.473973 & 1.624764 \\
\hline 31 & 6 & ๑. 823385 & 2.479540 & -0.659098 \\
\hline 32 & 6 & -1.366644 & 2.667486 & 0.704892 \\
\hline 33 & 1 & -1.978787 & 1.272590 & 2.225531 \\
\hline 34 & 1 & -0.292619 & 1.768277 & 2.345805 \\
\hline 35 & 6 & -0.095379 & 3.364898 & 0.207879 \\
\hline 36 & 1 & -1.962062 & 3.397239 & 1.271089 \\
\hline 37 & 1 & -1.993587 & 2.347931 & -0.136756 \\
\hline 38 & 1 & -0.364022 & 4.257850 & -0.376129 \\
\hline 39 & 1 & $\odot .476215$ & 3.708783 & 1.078535 \\
\hline 40 & 6 & $-\odot .248957$ & $-\odot .834748$ & 2.095745 \\
\hline 41 & 1 & -0.130710 & -1.852415 & 1.711733 \\
\hline 42 & 1 & $\odot .711576$ & -0.509107 & 2.521426 \\
\hline 43 & 1 & -0.968123 & -0.865090 & 2.934560 \\
\hline 44 & 6 & 2.146991 & 3.251333 & -0.908990 \\
\hline 45 & 1 & 1.935487 & 4.196636 & -1.426842 \\
\hline 46 & 1 & 2.653349 & 3.463632 & ๑. . 035186 \\
\hline 47 & 1 & 2.827434 & 2.667019 & -1.540858 \\
\hline 48 & 6 & ๑. 181098 & 2.254627 & -2.042979 \\
\hline 49 & 1 & ๑. 798371 & 1.614710 & -2.678617 \\
\hline 50 & 1 & $-\odot .808998$ & 1.801733 & -1.995520 \\
\hline 51 & 1 & ๑. 083569 & 3.231521 & -2.536900 \\
\hline
\end{tabular}

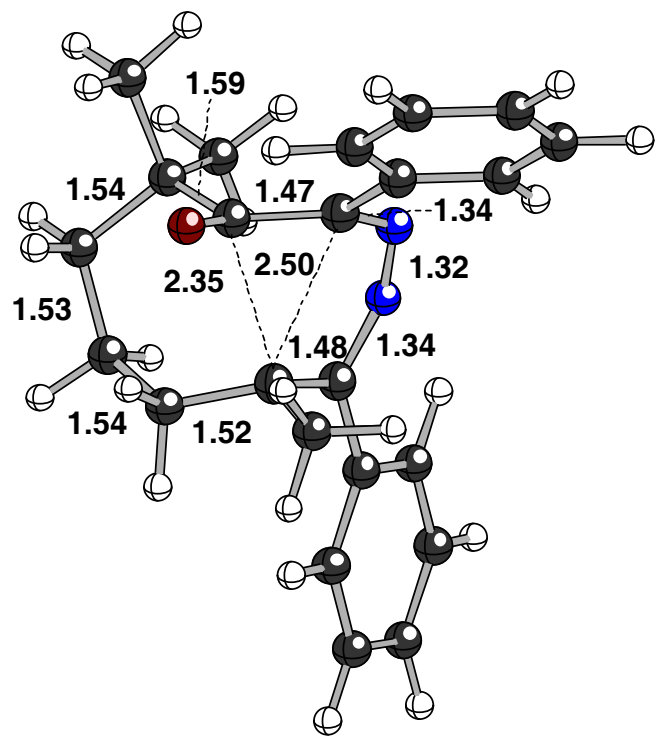




\section{2,2,6-Trimethylcyclohexanone Fragmentation TS (Table 2)}

$\mathrm{HF}=-1076.257075$ hartrees $(-675362.07713325 \mathrm{kcal} / \mathrm{mol})$

Imaginary Frequencies: 1 (-301.5678 1/cm)

Zero-point correction $=0.423634$ (Hartree/Particle)

Temperature 298.150 Kelvin. Pressure 1.00000 Atm.

Sum of electronic and thermal Free Energies = -1075.883556 hartrees (-675127.69022556 $\mathrm{kcal} / \mathrm{mol})$

Solvent Single Point: HF = -1076.33296 hartrees $(-675409.6958 \mathrm{kcal} / \mathrm{mol})$

Coordinates (from last standard orientation):

\begin{tabular}{|c|c|c|c|c|}
\hline \multirow{2}{*}{$\begin{array}{l}\text { Center } \\
\text { Number }\end{array}$} & \multirow{2}{*}{$\begin{array}{l}\text { Atomic } \\
\text { Number }\end{array}$} & \multicolumn{3}{|c|}{ Coordinates (Angstroms) } \\
\hline & & $\mathrm{X}$ & $\mathrm{Y}$ & Z \\
\hline$-5-1$ & 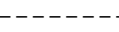 & 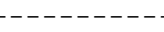 & ------- & ------- \\
\hline 1 & 6 & 0.954215 & 0.988007 & 0.197258 \\
\hline 2 & 6 & 1.448356 & -0.472941 & 0.277241 \\
\hline 3 & 6 & -1.019406 & 0.532105 & 1.420045 \\
\hline 4 & 6 & -1.411451 & -0.620129 & 0.687478 \\
\hline 5 & 8 & 1.474330 & 1.808578 & 0.992518 \\
\hline 6 & 7 & 0.748585 & -1.552524 & 0.515964 \\
\hline 7 & 7 & -0.606149 & -1.661466 & 0.445115 \\
\hline 8 & 6 & -2.803123 & -0.854164 & 0.161616 \\
\hline 9 & 6 & -3.012102 & -1.655112 & -0.976493 \\
\hline 10 & 6 & -3.936104 & $-\odot .373293$ & ๑. 839748 \\
\hline 11 & 6 & -4.299220 & -1.935440 & -1.436814 \\
\hline 12 & 1 & -2.141218 & -2.065518 & -1.476155 \\
\hline 13 & 6 & -5.227154 & $-\odot .651504$ & ๑. 383097 \\
\hline 14 & 1 & -3.802639 & $\odot .207602$ & 1.745161 \\
\hline 15 & 6 & -5.416285 & -1.428959 & -0.763122 \\
\hline 16 & 1 & -4.432546 & -2.553260 & -2.322120 \\
\hline 17 & 1 & -6.085887 & -0.267993 & 0.929422 \\
\hline 18 & 1 & -6.420012 & -1.644794 & -1.121676 \\
\hline 19 & 6 & 2.910065 & -0.717770 & 0.116382 \\
\hline 20 & 6 & 3.388764 & -2.037577 & $-\odot .064894$ \\
\hline 21 & 6 & 3.869611 & ๑. 317398 & ๑. 147123 \\
\hline 22 & 6 & 4.748560 & -2.306406 & -0.192308 \\
\hline 23 & 1 & 2.659804 & -2.839551 & -0.094789 \\
\hline 24 & 6 & 5.234276 & ๑. 042511 & ๑. . 017255 \\
\hline 25 & 1 & 3.539633 & 1.335744 & ๑. 308949 \\
\hline 26 & 6 & 5.689002 & -1.267054 & -0.153410 \\
\hline 27 & 1 & 5.079547 & -3.333582 & -0.333128 \\
\hline 28 & 1 & 5.946139 & ๑.864573 & 0.055662 \\
\hline 29 & 1 & 6.750904 & -1.476368 & -0.259509 \\
\hline 30 & 6 & -1.687481 & 1.888243 & 1.299110 \\
\hline 31 & 6 & 0.414357 & 1.566216 & -1.153815 \\
\hline 32 & 6 & -1.890571 & 2.389072 & -0.143062 \\
\hline 33 & 1 & -2.644411 & 1.960412 & 1.849785 \\
\hline 34 & 1 & -1.019355 & 2.602892 & 1.803016 \\
\hline 35 & 6 & -0.581715 & 2.723292 & -0.877982 \\
\hline 36 & 1 & -2.497250 & 3.307030 & -0.114628 \\
\hline 37 & 1 & -2.477643 & 1.663247 & -0.717265 \\
\hline 38 & 1 & -0.831948 & 3.175010 & -1.850351 \\
\hline 39 & 1 & -0.045333 & 3.487949 & -0.302389 \\
\hline 40 & 6 & -0.294632 & $\odot .330600$ & 2.733025 \\
\hline 41 & 1 & $\odot .577369$ & $\odot .994455$ & 2.816726 \\
\hline 42 & 1 & -0.977594 & 0.575088 & 3.566603 \\
\hline 43 & 1 & ๑. 039840 & -0.700385 & 2.872808 \\
\hline 44 & 6 & 1.641834 & 2.185307 & -1.877978 \\
\hline 45 & 1 & 2.130857 & 2.926567 & -1.239267 \\
\hline 46 & 1 & 2.379113 & 1.419677 & -2.142140 \\
\hline 47 & 1 & 1.319174 & 2.677502 & -2.805462 \\
\hline 48 & 6 & -0.190351 & 0.516437 & -2.100642 \\
\hline 49 & 1 & 0.488053 & -0.333473 & -2.231964 \\
\hline 50 & 1 & -1.144196 & ๑. 127018 & -1.749736 \\
\hline 51 & 1 & -0.358758 & $\odot .966422$ & -3.088380 \\
\hline
\end{tabular}

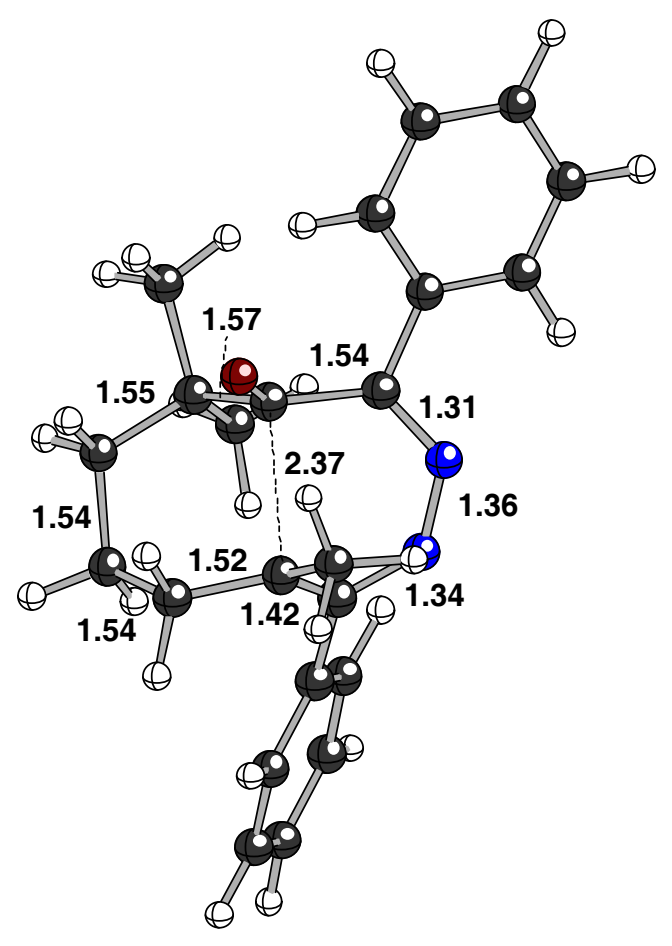




\section{2,2,6-Trimethylcyclohexanone 1,2-shift product (Table 2)}

$\mathrm{HF}=-1076.2959135$ hartrees $(-675386.448680385 \mathrm{kcal} / \mathrm{mol})$

Imaginary Frequencies: none found

Zero-point correction $=0.426414$ (Hartree/Particle)

Temperature 298.150 Kelvin. Pressure 1.00000 Atm.

Sum of electronic and thermal Free Energies = -1075.919800 hartrees $(-\mathbf{6 7 5 1 5 0 . 4 3 3 6 9 8 ~} \mathrm{kcal} / \mathrm{mol})$

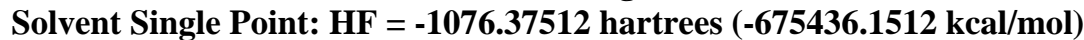

Coordinates (from last standard orientation):

\begin{tabular}{|c|c|c|c|c|}
\hline \multirow{2}{*}{$\begin{array}{l}\text { Center } \\
\text { Number }\end{array}$} & \multirow{2}{*}{$\begin{array}{l}\text { Atomic } \\
\text { Number }\end{array}$} & \multicolumn{3}{|c|}{ Coordinates (Angstroms) } \\
\hline & & $\mathrm{x}$ & $\mathrm{Y}$ & Z \\
\hline 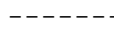 & 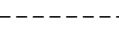 & -------1 & ------- & ------ \\
\hline 1 & 6 & 1.527370 & 1.475385 & -0.324322 \\
\hline 2 & 6 & 1.051436 & -0.020260 & -0.330830 \\
\hline 3 & 6 & -0.147571 & -0.470452 & 0.617888 \\
\hline 4 & 6 & -1.293314 & -0.452904 & -0.415117 \\
\hline 5 & 8 & 2.723216 & 1.685441 & -0.496953 \\
\hline 6 & 7 & $\odot .493712$ & -0.143003 & -1.729638 \\
\hline 7 & 7 & -0.791568 & -0.352354 & -1.651080 \\
\hline 8 & 6 & -2.698277 & -0.732036 & -0.228346 \\
\hline 9 & 6 & -3.573100 & $-\odot .831246$ & -1.353582 \\
\hline 10 & 6 & -3.310919 & $-\odot .905244$ & 1.044212 \\
\hline 11 & 6 & -4.929964 & -1.084536 & -1.208896 \\
\hline 12 & 1 & -3.142547 & -0.702993 & -2.340684 \\
\hline 13 & 6 & -4.677044 & -1.160403 & 1.178684 \\
\hline 14 & 1 & -2.712420 & -0.839220 & 1.944936 \\
\hline 15 & 6 & -5.510672 & -1.254948 & ๑. 060659 \\
\hline 16 & 1 & -5.551722 & -1.152413 & -2.100308 \\
\hline 17 & 1 & -5.092472 & -1.285711 & 2.177433 \\
\hline 18 & 1 & -6.573895 & -1.452421 & 0.169028 \\
\hline 19 & 6 & 2.302914 & -0.893635 & -0.169964 \\
\hline 20 & 6 & 2.651431 & -1.829832 & -1.150232 \\
\hline 21 & 6 & 3.112030 & -0.809165 & $\odot .974851$ \\
\hline 22 & 6 & 3.762391 & -2.665501 & - ๑. 988112 \\
\hline 23 & 1 & 2.037220 & -1.881598 & -2.042873 \\
\hline 24 & 6 & 4.218085 & -1.642178 & 1.145251 \\
\hline 25 & 1 & 2.882492 & $-\odot .074036$ & 1.740953 \\
\hline 26 & 6 & 4.550853 & -2.580131 & $\odot .161109$ \\
\hline 27 & 1 & 4.010877 & -3.384169 & -1.766202 \\
\hline 28 & 1 & 4.825956 & -1.553995 & 2.043110 \\
\hline 29 & 1 & 5.414859 & -3.228335 & ๑. 288711 \\
\hline 30 & 6 & -0.307881 & ๑. 396705 & 1.895238 \\
\hline 31 & 6 & ๑. 598619 & 2.729889 & -0.222541 \\
\hline 32 & 6 & -0.907349 & 1.800152 & 1.740923 \\
\hline 33 & 1 & -0.932477 & -0.162288 & 2.602152 \\
\hline 34 & 1 & 0.673886 & 0.473860 & 2.387719 \\
\hline 35 & 6 & ๑. 062071 & 2.868823 & 1. 229068 \\
\hline 36 & 1 & -1.263471 & 2.129310 & 2.727712 \\
\hline 37 & 1 & -1.793667 & 1.746523 & 1.099544 \\
\hline 38 & 1 & -0.436108 & 3.847665 & 1.287095 \\
\hline 39 & 1 & ๑. 923369 & 2.925595 & 1.912095 \\
\hline 40 & 6 & ๑. 019939 & -1.941431 & 1.094279 \\
\hline 41 & 1 & ๑. 251437 & -2.602795 & $\odot .255869$ \\
\hline 42 & 1 & ๑. 816931 & -2.047913 & 1.839466 \\
\hline 43 & 1 & -0.917438 & -2.285431 & 1.542933 \\
\hline 44 & 6 & 1.467789 & 3.976775 & -0.502563 \\
\hline 45 & 1 & 0.841808 & 4.874932 & -0.430586 \\
\hline 46 & 1 & 2.294108 & 4.066875 & ๑. 208081 \\
\hline 47 & 1 & 1.902938 & 3.935237 & -1.504875 \\
\hline 48 & 6 & $-\odot .566919$ & 2.747568 & -1.235215 \\
\hline 49 & 1 & -0.187898 & 2.690094 & -2.258957 \\
\hline 50 & 1 & -1.268269 & 1.926698 & -1.098321 \\
\hline 51 & 1 & -1.115730 & 3.692125 & -1.118035 \\
\hline
\end{tabular}

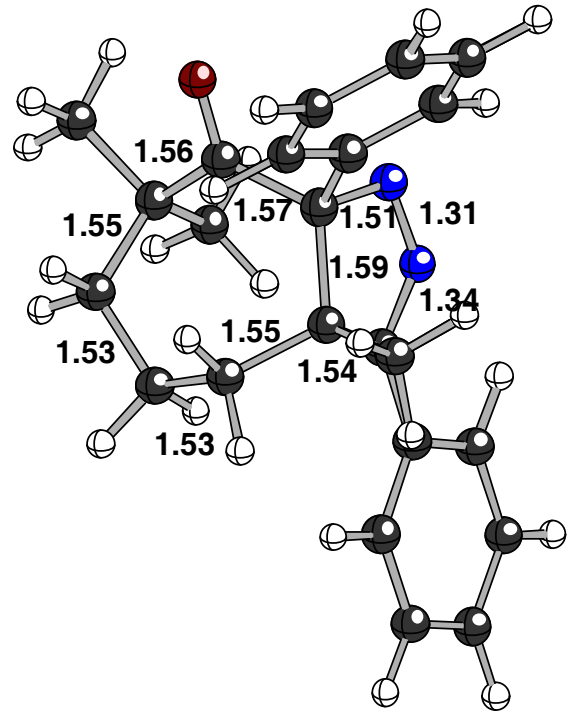




\section{2,2,6-Trimethylcyclohexanone Fragmentation product (Table 2)}

$\mathrm{HF}=-1076.2629891$ hartrees $(-675365.788290141 \mathrm{kcal} / \mathrm{mol})$

Imaginary Frequencies: none found

Zero-point correction $=0.424274$ (Hartree/Particle)

Temperature 298.150 Kelvin. Pressure 1.00000 Atm.

Sum of electronic and thermal Free Energies = -1075.889887 hartrees (-675131.66299137 $\mathrm{kcal} / \mathrm{mol})$

Solvent Single Point: HF $=-\mathbf{1 0 7 6 . 3 4 0 1 2 3}$ hartrees $(-675414.1903 \mathrm{kcal} / \mathrm{mol})$

Coordinates (from last standard orientation):

\begin{tabular}{|c|c|c|c|c|}
\hline \multirow{2}{*}{$\begin{array}{l}\text { Center } \\
\text { Number }\end{array}$} & \multirow{2}{*}{$\begin{array}{l}\text { Atomic } \\
\text { Number }\end{array}$} & \multicolumn{3}{|c|}{ Coordinates (Angstroms) } \\
\hline & & $\mathrm{X}$ & $\mathrm{Y}$ & Z \\
\hline$-5-1$ & 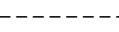 & 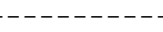 & ------- & ------- \\
\hline 1 & 6 & 1.053726 & 1.079220 & 0.071453 \\
\hline 2 & 6 & 1.455507 & -0.361727 & 0.345230 \\
\hline 3 & 6 & -1.267926 & ๑. 695928 & 1.647607 \\
\hline 4 & 6 & -1.449851 & -0.481717 & 0.955690 \\
\hline 5 & 8 & 1.481094 & 1.981272 & 0.796177 \\
\hline 6 & 7 & 0.736414 & -1.392284 & 0.794285 \\
\hline 7 & 7 & -0.545189 & -1.553072 & 1.042297 \\
\hline 8 & 6 & -2.708772 & -0.869389 & 0.240701 \\
\hline 9 & 6 & -2.685366 & -1.928868 & -0.689495 \\
\hline 10 & 6 & -3.966871 & $-\odot .315552$ & $\odot .550960$ \\
\hline 11 & 6 & -3.850858 & -2.375758 & -1.314376 \\
\hline 12 & 1 & -1.732044 & -2.404062 & -0.892567 \\
\hline 13 & 6 & -5.134080 & -0.756371 & -0.074723 \\
\hline 14 & 1 & -4.035882 & 0.457612 & 1.307245 \\
\hline 15 & 6 & -5.084387 & -1.787006 & -1.019671 \\
\hline 16 & 1 & -3.795228 & -3.191749 & -2.031502 \\
\hline 17 & 1 & -6.087613 & -0.303275 & 0.187756 \\
\hline 18 & 1 & -5.993211 & -2.134038 & -1.505474 \\
\hline 19 & 6 & 2.891160 & -0.668620 & 0.152009 \\
\hline 20 & 6 & 3.344103 & -2.015145 & ๑. 165129 \\
\hline 21 & 6 & 3.881019 & ๑. 322347 & -0.063802 \\
\hline 22 & 6 & 4.684005 & -2.339529 & -0.017888 \\
\hline 23 & 1 & 2.606181 & -2.791117 & 0.333253 \\
\hline 24 & 6 & 5.225096 & -0.011208 & -0.251320 \\
\hline 25 & 1 & 3.603196 & 1.369437 & $-\odot .050346$ \\
\hline 26 & 6 & 5.648412 & -1.342630 & -0.232101 \\
\hline 27 & 1 & 4.982501 & -3.386529 & ๑. . 001288 \\
\hline 28 & 1 & 5.949114 & $\odot .787350$ & -0.404045 \\
\hline 29 & 1 & 6.694998 & -1.599101 & -0.377091 \\
\hline 30 & 6 & -1.908670 & 2.029621 & 1.294809 \\
\hline 31 & 6 & 0.336342 & 1.530508 & -1.239963 \\
\hline 32 & 6 & -2.015848 & 2.350373 & -0.213029 \\
\hline 33 & 1 & -2.902209 & 2.155484 & 1.758732 \\
\hline 34 & 1 & -1.287206 & 2.811213 & 1.754207 \\
\hline 35 & 6 & -0.693417 & 2.662047 & -0.946764 \\
\hline 36 & 1 & -2.644671 & 3.248055 & -0.314605 \\
\hline 37 & 1 & -2.555181 & 1.553759 & -0.733221 \\
\hline 38 & 1 & $-\odot .961905$ & 3.090947 & -1.923983 \\
\hline 39 & 1 & -0.161662 & 3.447809 & -0.396694 \\
\hline 40 & 6 & -0.358493 & $\odot .725943$ & 2.852096 \\
\hline 41 & 1 & $\odot .581341$ & 1.263698 & 2.676980 \\
\hline 42 & 1 & -0.882265 & 1.251977 & 3.666704 \\
\hline 43 & 1 & -0.118029 & -0.286149 & 3.187786 \\
\hline 44 & 6 & 1.466407 & 2.168904 & -2.098467 \\
\hline 45 & 1 & 1.918597 & 3. 018187 & -1.577745 \\
\hline 46 & 1 & 2.255364 & 1.442510 & -2.319693 \\
\hline 47 & 1 & 1.051714 & 2.520227 & -3.052058 \\
\hline 48 & 6 & -0.266270 & ๑. 382820 & -2.059994 \\
\hline 49 & 1 & 0.484935 & -0.389763 & -2.254678 \\
\hline 50 & 1 & -1.107594 & $-\odot .093748$ & -1.562918 \\
\hline 51 & 1 & -0.620367 & $\odot .763450$ & -3.027355 \\
\hline
\end{tabular}

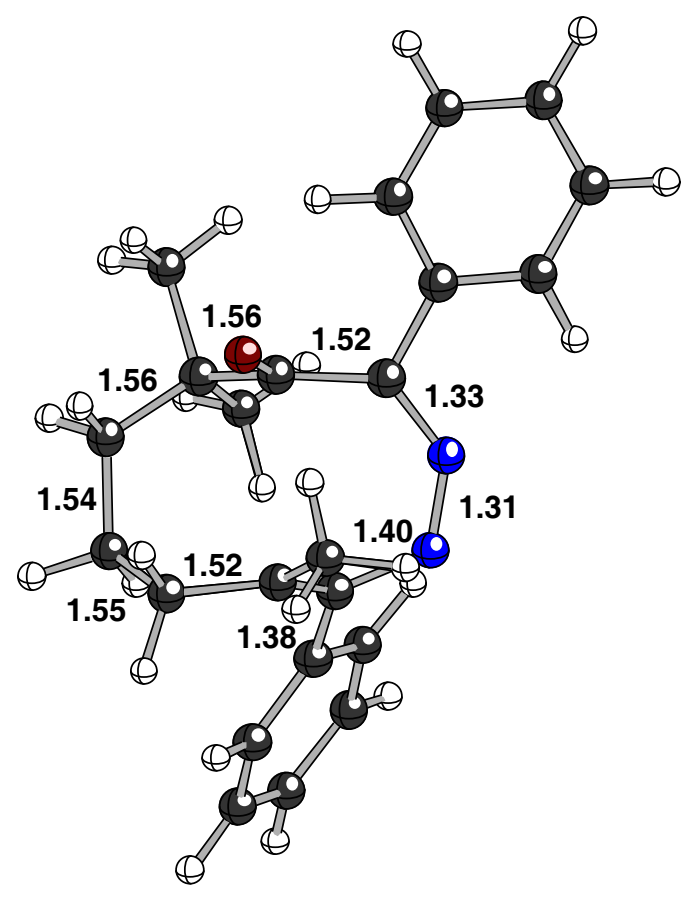




\section{Cycloheptanone reactant (Table 2)}

$\mathrm{HF}=-997.6492986$ hartrees $(-626034.911364486 \mathrm{kcal} / \mathrm{mol})$

Imaginary Frequencies: none found

Zero-point correction $=0.370942$ (Hartree/Particle)

Temperature 298.150 Kelvin. Pressure 1.00000 Atm.

Sum of electronic and thermal Free Energies = -997.327657 hartrees $(-625833.07804407 \mathrm{kcal} / \mathrm{mol})$

Solvent Single Point: HF = -997.7399346 hartrees $(-626091.7864 \mathrm{kcal} / \mathrm{mol})$

Coordinates (from last standard orientation):

\begin{tabular}{|c|c|c|c|c|}
\hline \multirow{2}{*}{$\begin{array}{l}\text { Center } \\
\text { Number }\end{array}$} & \multirow{2}{*}{$\begin{array}{l}\text { Atomic } \\
\text { Number }\end{array}$} & \multicolumn{3}{|c|}{ Coordinates (Angstroms) } \\
\hline & & $\mathrm{x}$ & $Y$ & Z \\
\hline --- & & $-\cdots-\cdots$ & ------ & $--.-1-$. \\
\hline 1 & 6 & -0.770033 & $\odot .819984$ & -0.173809 \\
\hline 2 & 6 & -1.503415 & $-\odot .566416$ & -0.079178 \\
\hline 3 & 6 & 0.569103 & 0.445101 & -0.951328 \\
\hline 4 & 1 & 0.199018 & 0.112190 & -1.936744 \\
\hline 5 & 6 & 1.222634 & $-\odot .756817$ & -0.298427 \\
\hline 6 & 8 & -1.449844 & 1.768401 & -0.837463 \\
\hline 7 & 7 & -0.879739 & -1.703066 & 0.027075 \\
\hline 8 & 7 & 0.512560 & -1.715722 & 0.202801 \\
\hline 9 & 6 & 2.698250 & $-\odot .951184$ & $-\odot .158980$ \\
\hline 10 & 6 & 3.240663 & -1.336341 & 1.079797 \\
\hline 11 & 6 & 3.572184 & -0.816207 & -1.251188 \\
\hline 12 & 6 & 4.610338 & -1.559129 & 1.227020 \\
\hline 13 & 1 & 2.566936 & -1.463761 & 1.920820 \\
\hline 14 & 6 & 4.942416 & -1.049538 & -1.108521 \\
\hline 15 & 1 & 3.173769 & $-\odot .550873$ & -2.225200 \\
\hline 16 & 6 & 5.470399 & -1.414796 & ๑.133145 \\
\hline 17 & 1 & 5.007451 & -1.847703 & 2.197333 \\
\hline 18 & 1 & 5.597185 & $-\odot .948776$ & -1.970781 \\
\hline 19 & 1 & 6.537661 & -1.587963 & ๑. 246271 \\
\hline 20 & 6 & -2.990756 & -0.678948 & -0.084321 \\
\hline 21 & 6 & -3.601203 & -1.780878 & 0.549810 \\
\hline 22 & 6 & -3.815663 & ๑. 279275 & -0.707169 \\
\hline 23 & 6 & -4.988092 & -1.930995 & 0.552497 \\
\hline 24 & 1 & -2.964844 & -2.513023 & 1.035128 \\
\hline 25 & 6 & -5.204914 & ๑.122717 & $-\odot .699186$ \\
\hline 26 & 1 & -3.312609 & 1.130362 & -1.159523 \\
\hline 27 & 6 & -5.799431 & -0.978473 & -0.075190 \\
\hline 28 & 1 & -5.437094 & -2.788204 & 1. 049887 \\
\hline 29 & 1 & -5.827714 & ๑.870589 & -1.185731 \\
\hline 30 & 1 & -6.881677 & -1.092111 & -0.071783 \\
\hline 31 & 6 & 1.442387 & 1.683375 & -1.207394 \\
\hline 32 & 6 & -0.462408 & 1.223242 & 1.323833 \\
\hline 33 & 6 & 1.941505 & 2.470791 & ๑. . 022046 \\
\hline 34 & 6 & -0.193508 & 2.721946 & 1.539366 \\
\hline 35 & 6 & ๑. 875455 & 3.391877 & 0.643467 \\
\hline 36 & 1 & -1.348128 & ๑. 970801 & 1.923332 \\
\hline 37 & 1 & 0.361635 & 0.623006 & 1.736359 \\
\hline 38 & 1 & -1.137997 & 3.254821 & 1.391263 \\
\hline 39 & 1 & ๑. 085491 & 2.851795 & 2.596781 \\
\hline 40 & 1 & 0.363305 & 3.899071 & -0.181718 \\
\hline 41 & 1 & 1.384334 & 4.172502 & 1.229715 \\
\hline 42 & 1 & 2.361333 & 1.793838 & 0.779313 \\
\hline 43 & 1 & 2.779932 & 3.101374 & -0.309051 \\
\hline 44 & 1 & ๑. 819753 & 2.355999 & -1.808461 \\
\hline 45 & 1 & 2.305812 & 1.408866 & -1.824370 \\
\hline
\end{tabular}

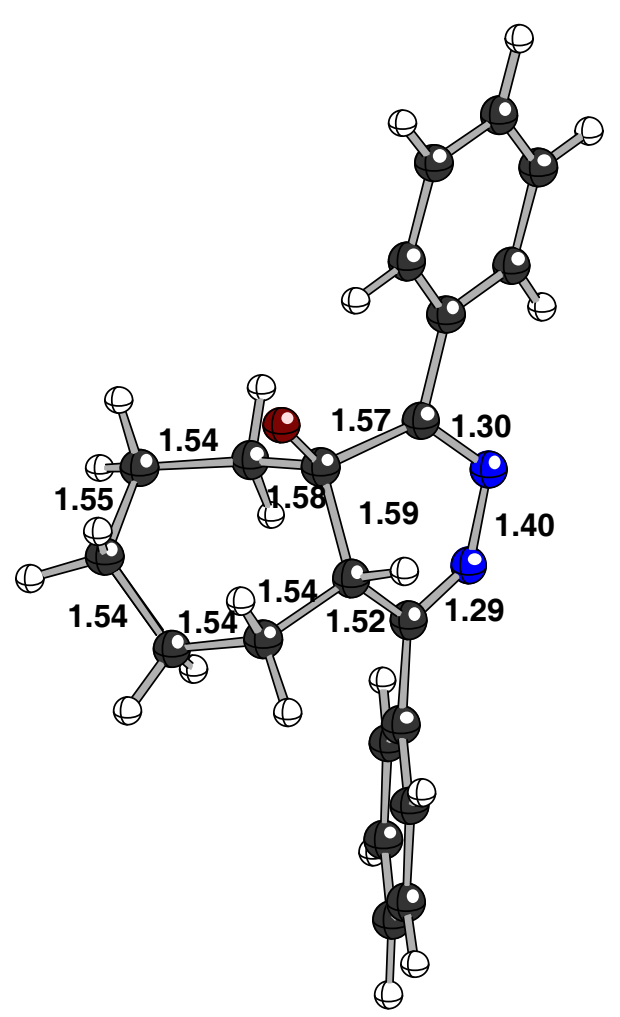




\section{Cycloheptanone 1,2-shift TS (Table 2)}

$\mathrm{HF}=-997.6097383$ hartrees $(-626010.086880633 \mathrm{kcal} / \mathrm{mol})$

Imaginary Frequencies: $1(-329.3166$ 1/cm)

Zero-point correction $=0.368704$ (Hartree/Particle)

Temperature 298.150 Kelvin. Pressure 1.00000 Atm.

Sum of electronic and thermal Free Energies $=\mathbf{- 9 9 7 . 2 8 9 4 0 5}$ hartrees $(-625809.07453155 \mathrm{kcal} / \mathrm{mol})$

Solvent Single Point: HF = $\mathbf{- 9 9 7 . 6 8 5 9 2 8 9}$ hartrees $(-626057.8972 \mathrm{kcal} / \mathbf{m o l})$

Coordinates (from last standard orientation):

\begin{tabular}{|c|c|c|c|c|}
\hline \multirow{2}{*}{$\begin{array}{l}\text { Center } \\
\text { Number }\end{array}$} & \multirow{2}{*}{$\begin{array}{l}\text { Atomic } \\
\text { Number }\end{array}$} & \multicolumn{3}{|c|}{ Coordinates (Angstroms) } \\
\hline & & $\mathrm{x}$ & $\mathrm{Y}$ & Z \\
\hline 1 & 6 & -1.143349 & 1.328610 & 0.316030 \\
\hline 2 & 6 & -1.474410 & $-\odot .081725$ & 0.601105 \\
\hline 3 & 6 & 0.372201 & 0.227195 & $-\odot .777746$ \\
\hline 4 & 1 & $-\odot .048547$ & -0.448494 & -1.539246 \\
\hline 5 & 6 & 1.168085 & -0.504398 & 0.252869 \\
\hline 6 & 8 & -1.797393 & 2.059251 & -0.488397 \\
\hline 7 & 7 & -0.748426 & -0.850176 & 1.434537 \\
\hline 8 & 7 & 0.586654 & -0.880355 & 1.387366 \\
\hline 9 & 6 & 2.531618 & -0.995907 & 0.033550 \\
\hline 10 & 6 & 3.351924 & -1.402236 & 1.119002 \\
\hline 11 & 6 & 3.094740 & -1.087099 & -1.261628 \\
\hline 12 & 6 & 4.648378 & -1.859038 & 0.916022 \\
\hline 13 & 1 & 2.934663 & -1.350981 & 2.118930 \\
\hline 14 & 6 & 4.394645 & -1.560633 & -1.460271 \\
\hline 15 & 1 & 2.500615 & -0.801254 & -2.122600 \\
\hline 16 & 6 & 5.189179 & -1.945933 & -0.378023 \\
\hline 17 & 1 & 5.249143 & -2.155215 & 1.773946 \\
\hline 18 & 1 & 4.786930 & -1.625783 & -2.473292 \\
\hline 19 & 1 & 6.203988 & -2.303461 & $-\odot .532855$ \\
\hline 20 & 6 & -2.762208 & -0.701561 & 0.153193 \\
\hline 21 & 6 & -2.997905 & -2.062122 & 0.444339 \\
\hline 22 & 6 & -3.763659 & 0.010290 & -0.538222 \\
\hline 23 & 6 & -4.187233 & -2.684989 & ๑.070758 \\
\hline 24 & 1 & -2.227997 & -2.605416 & $\odot .981403$ \\
\hline 25 & 6 & -4.953870 & $-\odot .620698$ & $-\odot .913199$ \\
\hline 26 & 1 & -3.574206 & 1.048698 & -0.781347 \\
\hline 27 & 6 & -5.175624 & -1.967072 & -0.613401 \\
\hline 28 & 1 & -4.343602 & -3.734242 & ๑.311691 \\
\hline 29 & 1 & -5.711837 & -0.051346 & -1.446861 \\
\hline 30 & 1 & -6.102644 & -2.452827 & $-\odot .910258$ \\
\hline 31 & 6 & 0.914252 & 1.456657 & -1.494465 \\
\hline 32 & 6 & -0.317392 & 2.030560 & 1.412043 \\
\hline 33 & 6 & 1.683319 & 2.546923 & -0.708867 \\
\hline 34 & 6 & 0.033424 & 3.488988 & 1.103737 \\
\hline 35 & 6 & $\odot .818880$ & 3.722393 & -0.207088 \\
\hline 36 & 1 & -0.938453 & 1.996410 & 2.320546 \\
\hline 37 & 1 & 0.587716 & 1.476577 & 1.663652 \\
\hline 38 & 1 & $-\odot .884867$ & 4.085927 & 1.070424 \\
\hline 39 & 1 & $\odot .623835$ & 3.862297 & 1.953762 \\
\hline 40 & 1 & $\odot .108629$ & 3.975865 & -1.001316 \\
\hline 41 & 1 & 1.466599 & 4.601614 & -0.072346 \\
\hline 42 & 1 & 2.252687 & 2.099474 & 0.115972 \\
\hline 43 & 1 & 2.433783 & 2.964482 & -1.394079 \\
\hline 44 & 1 & 0.062338 & 1.929747 & $-2.0 \odot 4959$ \\
\hline 45 & 1 & 1.582809 & 1.098406 & -2.295736 \\
\hline
\end{tabular}

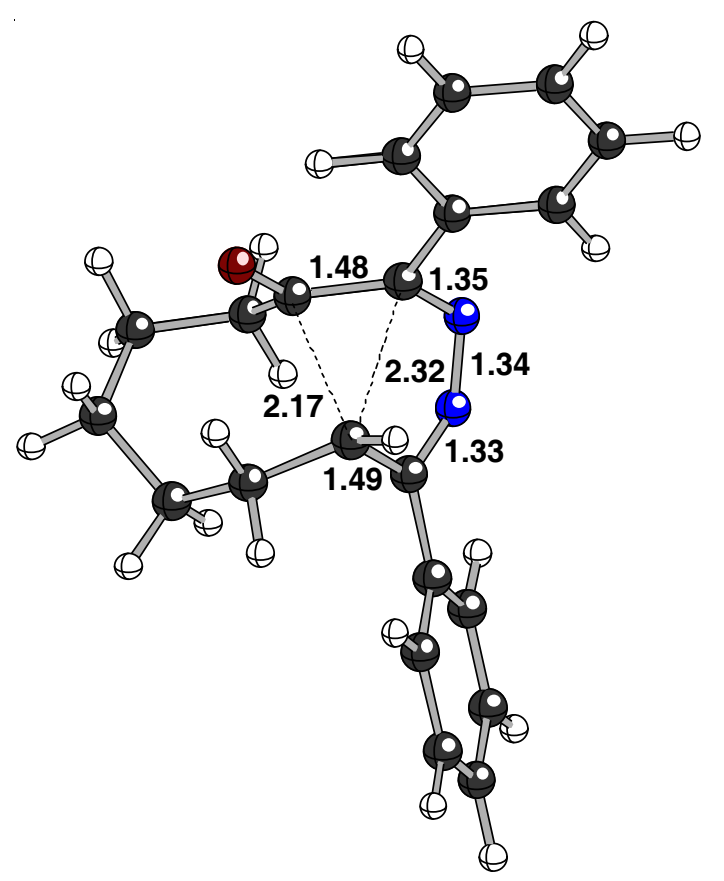




\section{Cycloheptanone Fragmentation TS (Table 2)}

$\mathrm{HF}=-997.628431$ hartrees $(-626021.81673681 \mathrm{kcal} / \mathrm{mol})$

Imaginary Frequencies: 1 (-357.3449 1/cm)

Zero-point correction $=0.369254$ (Hartree/Particle)

Temperature 298.150 Kelvin. Pressure 1.00000 Atm.

Sum of electronic and thermal Free Energies = -997.307166 hartrees $(-625820.21973666 \mathrm{kcal} / \mathrm{mol})$

Solvent Single Point: HF = -997.7080088 hartrees $(-626071.7526$ kcal $/ \mathrm{mol})$

Coordinates (from last standard orientation):

\begin{tabular}{|c|c|c|c|c|}
\hline \multirow{2}{*}{$\begin{array}{l}\text { Center } \\
\text { Number }\end{array}$} & \multirow{2}{*}{$\begin{array}{l}\text { Atomic } \\
\text { Number }\end{array}$} & \multicolumn{3}{|c|}{ Coordinates (Angstroms) } \\
\hline & & $\mathrm{x}$ & $\mathrm{Y}$ & Z \\
\hline$-\cdots--$ & & $-\cdots-\cdots$ & -------- & $--.-1-$. \\
\hline 1 & 6 & -1.044427 & 1.018992 & ๑. $0 \odot \odot 197$ \\
\hline 2 & 6 & -1.662242 & $-\odot .387921$ & -0.161217 \\
\hline 3 & 6 & 0.737246 & 0.469629 & -1.267658 \\
\hline 4 & 1 & -0.114347 & 0.292510 & -1.916323 \\
\hline 5 & 6 & 1.170805 & -0.676458 & -0.577195 \\
\hline 6 & 8 & -1.505981 & 1.979159 & -0.664247 \\
\hline 7 & 7 & -1.012863 & -1.511850 & -0.316328 \\
\hline 8 & 7 & 0.340355 & -1.663768 & -0.202880 \\
\hline 9 & 6 & 2.594499 & $-\odot .955784$ & $-\odot .199854$ \\
\hline 10 & 6 & 2.897927 & -1.674179 & ๑. 970762 \\
\hline 11 & 6 & 3.656721 & -0.579975 & -1.037469 \\
\hline 12 & 6 & 4.217235 & -1.980913 & 1.304682 \\
\hline 13 & 1 & 2.073760 & -1.995927 & 1.598945 \\
\hline 14 & 6 & 4.979864 & -0.890323 & -0.709572 \\
\hline 15 & 1 & 3.436675 & -0.057343 & -1.962825 \\
\hline 16 & 6 & 5.267863 & -1.587551 & ๑. 466908 \\
\hline 17 & 1 & 4.428534 & -2.531457 & 2.218732 \\
\hline 18 & 1 & 5.785111 & $-\odot .592135$ & -1.377191 \\
\hline 19 & 1 & 6.296576 & -1.827401 & 0.725547 \\
\hline 20 & 6 & -3.139109 & -0.511425 & -0.055262 \\
\hline 21 & 6 & -3.763103 & -1.773529 & -0.201251 \\
\hline 22 & 6 & -3.974896 & ๑. 599762 & 0.189536 \\
\hline 23 & 6 & -5.144186 & -1.913174 & -0.112435 \\
\hline 24 & 1 & -3.127355 & -2.632679 & -0.384012 \\
\hline 25 & 6 & -5.362590 & $\odot .452955$ & ๑. 281228 \\
\hline 26 & 1 & -3.533139 & 1.584788 & 0.279107 \\
\hline 27 & 6 & -5.961916 & -0.799391 & ๑.131801 \\
\hline 28 & 1 & -5.590547 & -2.898775 & -0.230158 \\
\hline 29 & 1 & -5.977330 & 1.331360 & 0.466778 \\
\hline 30 & 1 & -7.041358 & -0.910143 & ๑. 203938 \\
\hline 31 & 6 & 1.405826 & 1.792964 & -1.547528 \\
\hline 32 & 6 & -0.420242 & 1.292913 & 1.370806 \\
\hline 33 & 6 & 2.331331 & 2.430432 & -0.482204 \\
\hline 34 & 6 & 0.509723 & 2.517899 & 1.437718 \\
\hline 35 & 6 & 1.967767 & 2.250302 & 1.006137 \\
\hline 36 & 1 & -1.278496 & 1.456832 & 2.044749 \\
\hline 37 & 1 & 0.092804 & ๑. 395522 & 1.737600 \\
\hline 38 & 1 & 0.525371 & 2.869185 & 2.478735 \\
\hline 39 & 1 & 0.067040 & 3.325761 & 0.841312 \\
\hline 40 & 1 & 2.243292 & 1.237851 & 1.323661 \\
\hline 41 & 1 & 2.628156 & 2.924489 & 1.571977 \\
\hline 42 & 1 & 2.373037 & 3.507881 & -0.703474 \\
\hline 43 & 1 & 3.358425 & 2.063675 & -0.603914 \\
\hline 44 & 1 & 0.564565 & 2.484038 & -1.708309 \\
\hline 45 & 1 & 1.960050 & 1.796657 & -2.505212 \\
\hline
\end{tabular}

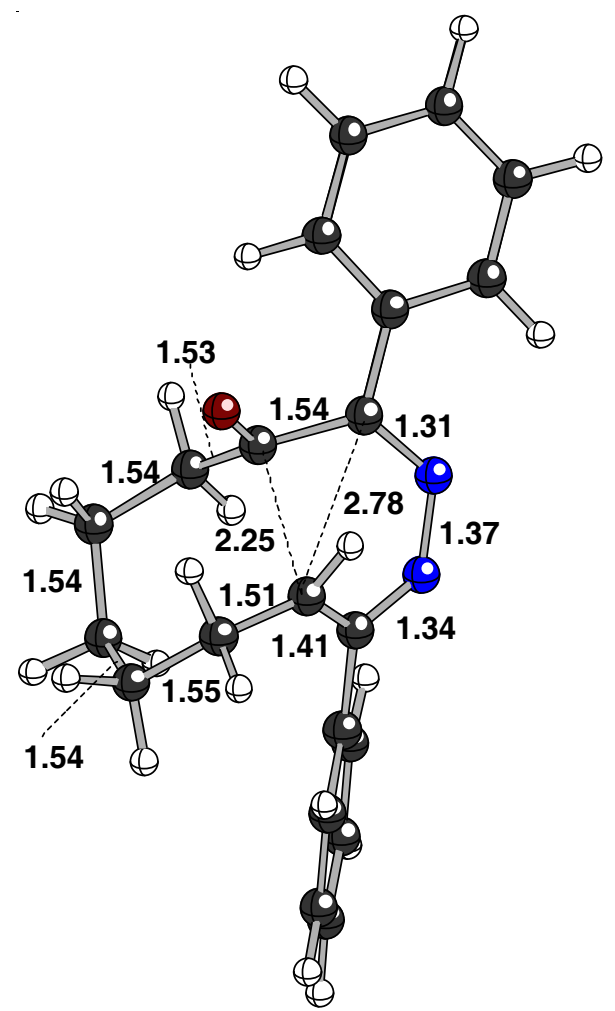




\section{Cycloheptanone 1,2-shift product (Table 2)}

$\mathrm{HF}=-997.6705858$ hartrees $(-626048.269295358 \mathrm{kcal} / \mathrm{mol})$

Imaginary Frequencies: none found

Zero-point correction $=0.371512$ (Hartree/Particle)

Temperature 298.150 Kelvin. Pressure 1.00000 Atm.

Sum of electronic and thermal Free Energies $=\mathbf{- 9 9 7 . 3 4 8 1 5 5}$ hartrees $(-625845.94074405 \mathrm{kcal} / \mathrm{mol})$

Solvent Single Point: HF = -997.7515997 hartrees $(-626099.1063 \mathrm{kcal} / \mathrm{mol})$

Coordinates (from last standard orientation):

\begin{tabular}{|c|c|c|c|c|}
\hline \multirow{2}{*}{$\begin{array}{l}\text { Center } \\
\text { Number }\end{array}$} & \multirow{2}{*}{$\begin{array}{l}\text { Atomic } \\
\text { Number }\end{array}$} & \multicolumn{3}{|c|}{ Coordinates (Angstroms) } \\
\hline & & $\mathrm{x}$ & $\mathrm{Y}$ & Z \\
\hline 1 & 6 & 1.499709 & 1.470719 & $-\odot .827141$ \\
\hline 2 & 6 & 1.120195 & 0.010910 & -0.506652 \\
\hline 3 & 6 & -0.069182 & -0.148771 & 0.521462 \\
\hline 4 & 1 & 0.139215 & -1.072675 & 1.089629 \\
\hline 5 & 6 & -1.218089 & -0.430777 & -0.449173 \\
\hline 6 & 8 & 2.643670 & $1.9 \odot 4858$ & -0.743383 \\
\hline 7 & 7 & 0.545577 & -0.441672 & -1.831455 \\
\hline 8 & 7 & -0.740783 & -0.617417 & -1.687247 \\
\hline 9 & 6 & -2.583723 & -0.766696 & -0.133934 \\
\hline 10 & 6 & -3.538270 & -1.009690 & -1.166990 \\
\hline 11 & 6 & -3.062749 & -0.892921 & 1.199042 \\
\hline 12 & 6 & -4.854685 & -1.342901 & -0.881397 \\
\hline 13 & 1 & -3.202575 & -0.930253 & -2.195526 \\
\hline 14 & 6 & -4.390098 & -1.228148 & 1.475338 \\
\hline 15 & 1 & -2.383601 & -0.740382 & 2.030444 \\
\hline 16 & 6 & -5.308090 & -1.455019 & 0.445986 \\
\hline 17 & 1 & -5.546260 & -1.518413 & -1.704006 \\
\hline 18 & 1 & -4.706520 & -1.316180 & 2.513555 \\
\hline 19 & 1 & -6.341529 & -1.711444 & 0.664122 \\
\hline 20 & 6 & 2.327721 & -0.824387 & $-\odot .099149$ \\
\hline 21 & 6 & 2.595606 & -2.040739 & -0.743305 \\
\hline 22 & 6 & 3.163280 & -0.442156 & 0.964175 \\
\hline 23 & 6 & 3.656297 & -2.855309 & -0.333546 \\
\hline 24 & 1 & 1.958365 & -2.326951 & -1.573270 \\
\hline 25 & 6 & 4.221703 & -1.254388 & 1.377577 \\
\hline 26 & 1 & 2.996943 & 0.505876 & 1.463868 \\
\hline 27 & 6 & 4.475028 & -2.468245 & 0.730268 \\
\hline 28 & 1 & 3.841030 & -3.795358 & -0.849028 \\
\hline 29 & 1 & 4.854750 & -0.933922 & 2.202179 \\
\hline 30 & 1 & 5.300414 & -3.100270 & 1.050165 \\
\hline 31 & 6 & -0.235524 & 0.927316 & 1.633284 \\
\hline 32 & 6 & 0.394392 & 2.366881 & -1.380272 \\
\hline 33 & 6 & $-\odot .907774$ & 2.295437 & 1.352450 \\
\hline 34 & 6 & 0.221821 & 3.664686 & -0.574567 \\
\hline 35 & 6 & 0.033475 & 3.451992 & 0.938656 \\
\hline 36 & 1 & 0.680044 & 2.612619 & -2.410460 \\
\hline 37 & 1 & $-\odot .549553$ & 1.824207 & -1.431344 \\
\hline 38 & 1 & 1.088191 & 4.317780 & -0.735270 \\
\hline 39 & 1 & $-\odot .653278$ & 4.192353 & -0.979297 \\
\hline $4 \odot$ & 1 & 1.015296 & 3.286826 & 1.402546 \\
\hline 41 & 1 & -0.342622 & 4.392012 & 1.365989 \\
\hline 42 & 1 & -1.715584 & 2.170443 & $\odot .620535$ \\
\hline 43 & 1 & -1.398631 & 2.593771 & 2.288010 \\
\hline 44 & 1 & 0.752367 & 1.107801 & 2.081821 \\
\hline 45 & 1 & -0.815630 & ๑. 450151 & 2.430646 \\
\hline
\end{tabular}

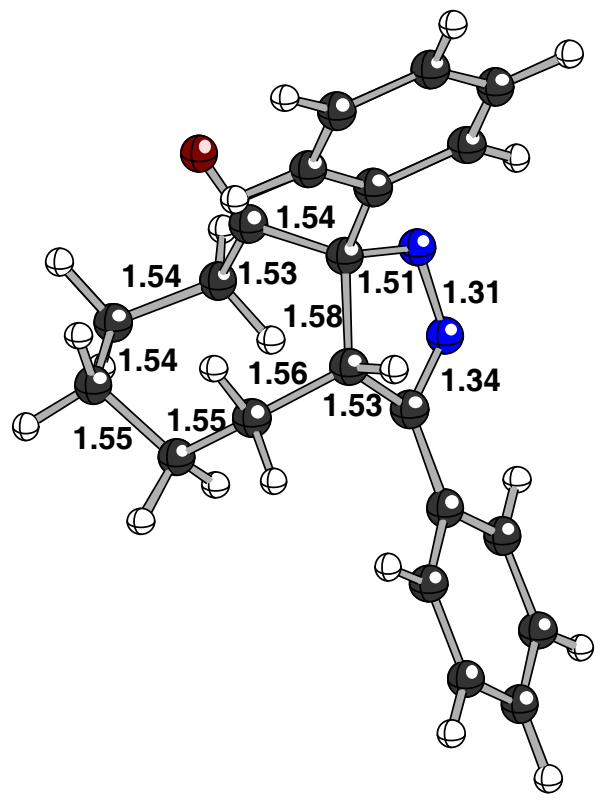




\section{Cycloheptanone Fragmentation product (Table 2)}

$\mathrm{HF}=-997.6350416$ hartrees $(-626025.964954416 \mathrm{kcal} / \mathrm{mol})$

Imaginary Frequencies: none found

Zero-point correction $=0.369501$ (Hartree/Particle)

Temperature 298.150 Kelvin. Pressure 1.00000 Atm.

Sum of electronic and thermal Free Energies = -997.315638 hartrees $(-625825.53600138 \mathrm{kcal} / \mathrm{mol})$

Solvent Single Point: HF = -997.7134972 hartrees $(-626075.1966$ kcal $/ \mathrm{mol})$

Coordinates (from last standard orientation):

\begin{tabular}{|c|c|c|c|c|}
\hline \multirow{2}{*}{$\begin{array}{l}\text { Center } \\
\text { Number }\end{array}$} & \multirow{2}{*}{$\begin{array}{l}\text { Atomic } \\
\text { Number }\end{array}$} & \multicolumn{3}{|c|}{ Coordinates (Angstroms) } \\
\hline & & $\mathrm{X}$ & $\mathrm{Y}$ & Z \\
\hline$x^{2}+2=$ & 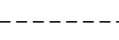 & $--\cdots---\cdots$ & ------ & ------ \\
\hline 1 & 6 & -1.258320 & 1.165724 & 0.221288 \\
\hline 2 & 6 & -1.763979 & -0.208331 & -0.091798 \\
\hline 3 & 6 & 0.982489 & ๑. 609997 & -1.426782 \\
\hline 4 & 1 & -0.028075 & 0.703039 & -1.809913 \\
\hline 5 & 6 & 1.214288 & -0.529542 & -0.710473 \\
\hline 6 & 8 & -1.695020 & 2.179207 & -0.329059 \\
\hline 7 & 7 & -1.036784 & -1.308543 & -0.329095 \\
\hline 8 & 7 & 0.249742 & -1.521090 & -0.459676 \\
\hline 9 & 6 & 2.569972 & $-\odot .969983$ & $-\odot .256586$ \\
\hline 10 & 6 & 2.705465 & -1.762603 & ๑. 898610 \\
\hline 11 & 6 & 3.733430 & -0.678943 & $-\odot .989679$ \\
\hline 12 & 6 & 3.957610 & -2.203030 & 1.329984 \\
\hline 13 & 1 & 1.804821 & -2.038977 & 1.436920 \\
\hline 14 & 6 & 4.988465 & -1.122643 & -0.565026 \\
\hline 15 & 1 & 3.647144 & -0.118633 & -1.914848 \\
\hline 16 & 6 & 5.109362 & -1.881196 & ๑. 603255 \\
\hline 17 & 1 & 4.034894 & -2.807155 & 2.231170 \\
\hline 18 & 1 & 5.871531 & -0.885555 & -1.154319 \\
\hline 19 & 1 & 6.084940 & -2.228906 & 0.934679 \\
\hline 20 & 6 & -3.226917 & -0.432342 & - ๑. . 056338 \\
\hline 21 & 6 & -3.758221 & -1.735827 & -0.240385 \\
\hline 22 & 6 & -4.166144 & ๑. 601582 & ๑.181271 \\
\hline 23 & 6 & -5.125799 & -1.984333 & -0.179021 \\
\hline 24 & 1 & -3.058362 & -2.541490 & $-\odot .429696$ \\
\hline 25 & 6 & -5.538054 & $\odot .344740$ & ๑. 238137 \\
\hline 26 & 1 & -3.819086 & 1.621037 & 0.298403 \\
\hline 27 & 6 & -6.039868 & -0.947836 & ๑. . 061159 \\
\hline 28 & 1 & -5.485876 & -3.001688 & -0.323715 \\
\hline 29 & 1 & -6.221320 & 1.172918 & ๑. 418347 \\
\hline 30 & 1 & -7.108737 & -1.143217 & 0.102015 \\
\hline 31 & 6 & 1.800759 & 1.868338 & -1.622014 \\
\hline 32 & 6 & -0.293247 & 1.320697 & 1.396636 \\
\hline 33 & 6 & 2.649847 & 2.411291 & -0.438389 \\
\hline 34 & 6 & 0.663746 & 2.535791 & 1.331992 \\
\hline 35 & 6 & 2.140806 & 2.200838 & 1.004634 \\
\hline 36 & 1 & -0.975400 & 1.436076 & 2.255174 \\
\hline 37 & 1 & ๑. 245320 & ๑. 385604 & 1.570449 \\
\hline 38 & 1 & 0.642087 & 3.035509 & 2.308592 \\
\hline 39 & 1 & ๑. 251172 & 3.258384 & $\odot .617541$ \\
\hline 40 & 1 & 2.340531 & 1.167088 & 1.304485 \\
\hline 41 & 1 & 2.788521 & 2.818398 & 1.644003 \\
\hline 42 & 1 & 2.779779 & 3.489985 & -0.613709 \\
\hline 43 & 1 & 3.658091 & 1.981469 & -0.478129 \\
\hline 44 & 1 & 1.073551 & 2.646271 & -1.886966 \\
\hline 45 & 1 & 2.469787 & 1.809284 & -2.499466 \\
\hline
\end{tabular}

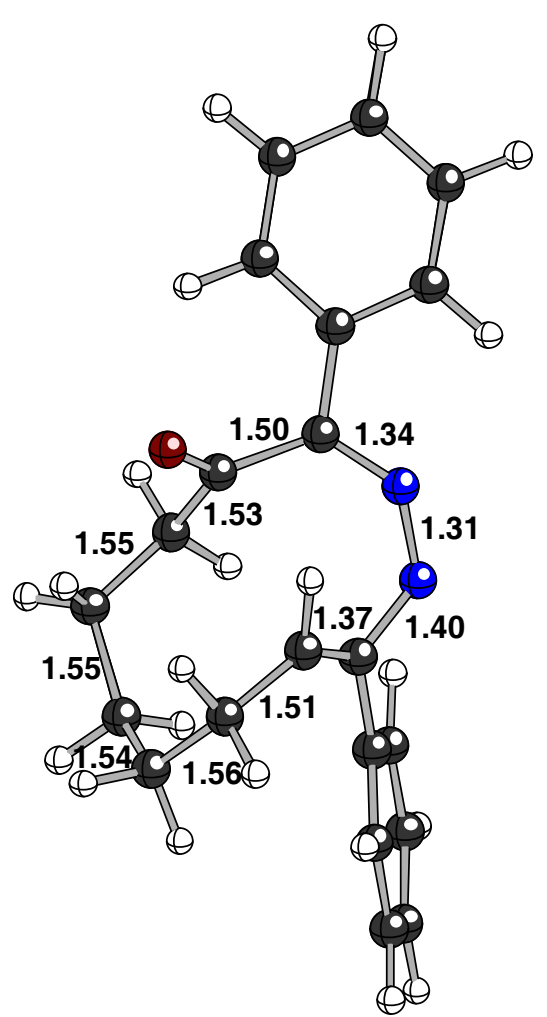




\section{Cyclooctanone reactant (Table 2)}

$\mathrm{HF}=-1036.9581996$ hartrees $(-650701.639830996 \mathrm{kcal} / \mathrm{mol})$

Imaginary Frequencies: none found

Zero-point correction $=0.399563$ (Hartree/Particle)

Temperature 298.150 Kelvin. Pressure 1.00000 Atm.

Sum of electronic and thermal Free Energies $=\mathbf{- 1 0 3 6 . 6 0 8 7 9 7}$ hartrees $(-650482.38620547 \mathrm{kcal} / \mathrm{mol})$

Solvent Single Point: HF = -1037.044202 hartrees $(-650755.6071 \mathrm{kcal} / \mathrm{mol})$

Coordinates (from last standard orientation):

\begin{tabular}{|c|c|c|c|c|}
\hline \multirow{2}{*}{$\begin{array}{l}\text { Center } \\
\text { Number }\end{array}$} & \multirow{2}{*}{$\begin{array}{l}\text { Atomic } \\
\text { Number }\end{array}$} & \multicolumn{3}{|c|}{ Coordinates (Angstroms) } \\
\hline & & $\mathrm{x}$ & $\mathrm{Y}$ & Z \\
\hline 1 & 6 & -0.776465 & 0.606339 & $-\odot .256291$ \\
\hline 2 & 6 & -1.434091 & -0.801026 & $-\odot .085676$ \\
\hline 3 & 6 & 0.720973 & 0.342746 & -0.739302 \\
\hline 4 & 1 & 0.606261 & 0.201296 & -1.827094 \\
\hline 5 & 6 & 1.307840 & -0.930517 & -0.150780 \\
\hline 6 & 8 & -1.435279 & 1.332506 & -1.186196 \\
\hline 7 & 7 & -0.791485 & -1.912486 & 0.113320 \\
\hline 8 & 7 & 0.595261 & -1.908207 & 0.304176 \\
\hline 9 & 6 & 2.781657 & -1.178240 & -0.077283 \\
\hline 10 & 6 & 3.354766 & -1.716601 & 1.087898 \\
\hline 11 & 6 & 3.620432 & -0.943504 & -1.180671 \\
\hline 12 & 6 & 4.720428 & -1.995701 & 1.153822 \\
\hline 13 & 1 & 2.707202 & -1.920251 & 1.934499 \\
\hline 14 & 6 & 4.985605 & -1.234252 & -1.121157 \\
\hline 15 & 1 & 3.195304 & -0.550685 & -2.099684 \\
\hline 16 & 6 & 5.544994 & -1.755383 & ๑. 049075 \\
\hline 17 & 1 & 5.142635 & -2.404411 & 2.068870 \\
\hline 18 & 1 & 5.612044 & -1.053975 & -1.991460 \\
\hline 19 & 1 & 6.608744 & -1.973878 & ๑. 099057 \\
\hline 20 & 6 & -2.919694 & -0.969628 & -0.126141 \\
\hline 21 & 6 & -3.500663 & -1.996916 & 0.646958 \\
\hline 22 & 6 & -3.772647 & -0.151540 & -0.894166 \\
\hline 23 & 6 & -4.880187 & -2.205153 & 0.652879 \\
\hline 24 & 1 & -2.846539 & -2.627372 & 1.239400 \\
\hline 25 & 6 & -5.154949 & -0.362340 & -0.880000 \\
\hline 26 & 1 & -3.300843 & 0.638979 & -1.468850 \\
\hline 27 & 6 & -5.717905 & -1.386716 & -0.113060 \\
\hline 28 & 1 & -5.302044 & -3.002880 & 1.260646 \\
\hline 29 & 1 & -5.796192 & 0.279701 & -1.480375 \\
\hline 30 & 1 & -6.794429 & -1.545313 & -0.109069 \\
\hline 31 & 6 & 1.658593 & 1.555546 & $-\odot .50755 \odot$ \\
\hline 32 & 6 & -0.845765 & 1.247807 & 1.203257 \\
\hline 33 & 6 & 1.092954 & 2.908347 & -1.001909 \\
\hline 34 & 1 & -0.032241 & ๑.869966 & 1.842795 \\
\hline 35 & 1 & -1.774254 & 0.866432 & 1.645791 \\
\hline 36 & 6 & -0.936783 & 2.789178 & 1.283933 \\
\hline 37 & 6 & 1.056290 & 4.001018 & 0.078333 \\
\hline 38 & 1 & 1.689228 & 3.273092 & -1.850725 \\
\hline 39 & 1 & $\odot .075348$ & 2.734112 & -1.369067 \\
\hline 40 & 6 & 0.382880 & 3.584115 & 1.403331 \\
\hline 41 & 1 & -1.512495 & 3.119133 & $\odot .409421$ \\
\hline 42 & 1 & -1.540377 & 3.029382 & 2.170204 \\
\hline 43 & 1 & 2.075104 & 4.355949 & 0.305411 \\
\hline 44 & 1 & 0.517861 & 4.867757 & -0.333198 \\
\hline 45 & 1 & 1. 090408 & 3.005698 & 2.014657 \\
\hline 46 & 1 & 0.193246 & 4.503017 & 1.977894 \\
\hline 47 & 1 & 1.889656 & 1.609014 & 0.563485 \\
\hline 48 & 1 & 2.622598 & 1.364565 & -0.988504 \\
\hline
\end{tabular}

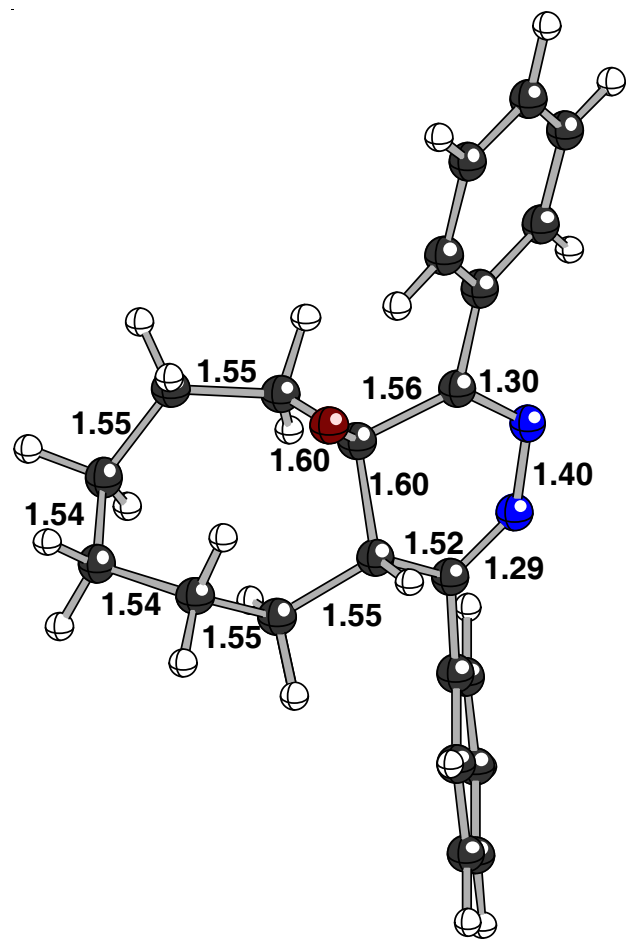




\section{Cyclooctanone Electrocyclic TS (Table 2)}

$\mathrm{HF}=-1036.9179349$ hartrees $(-650676.373329099 \mathrm{kcal} / \mathrm{mol})$

Imaginary Frequencies: $1(-164.58911 / \mathrm{cm})$

Zero-point correction $=0.397249$ (Hartree/Particle)

Temperature 298.150 Kelvin. Pressure 1.00000 Atm.

Sum of electronic and thermal Free Energies $=-\mathbf{1 0 3 6 . 5 7 0 9 3 0}$ hartrees $(-650458.6242843 \mathrm{kcal} / \mathrm{mol})$

Solvent Single Point: HF = -1036.991444 hartrees $(-650722.5011$ kcal $/ \mathrm{mol})$

Coordinates (from last standard orientation):

\begin{tabular}{|c|c|c|c|c|}
\hline \multirow{2}{*}{$\begin{array}{l}\text { Center } \\
\text { Number }\end{array}$} & \multirow{2}{*}{$\begin{array}{l}\text { Atomic } \\
\text { Number }\end{array}$} & \multicolumn{3}{|c|}{ Coordinates (Angstroms) } \\
\hline & & $\mathrm{x}$ & $\mathrm{Y}$ & Z \\
\hline 1 & 6 & 1.340814 & $\odot .999552$ & 0.047748 \\
\hline 2 & 6 & 1.356222 & -0.432632 & -0.357866 \\
\hline 3 & 6 & -1.016103 & 0.622171 & 0.609220 \\
\hline 4 & 1 & -1.167743 & 0.424352 & 1.679277 \\
\hline 5 & 6 & -1.485835 & -0.403176 & -0.279922 \\
\hline 6 & 8 & 1.912696 & 1.432032 & 1.075738 \\
\hline 7 & 7 & 0.537040 & -1.011474 & -1.227307 \\
\hline 8 & 7 & $-\odot .784030$ & -0.886366 & -1.338698 \\
\hline 9 & 6 & -2.772802 & -1.090636 & -0.054276 \\
\hline 10 & 6 & -3.153482 & -2.228332 & -0.807802 \\
\hline 11 & 6 & -3.690107 & -0.623603 & 0.915782 \\
\hline 12 & 6 & -4.387834 & -2.842515 & -0.615381 \\
\hline 13 & 1 & -2.448974 & -2.609911 & -1.538116 \\
\hline 14 & 6 & -4.913602 & -1.263085 & 1.128782 \\
\hline 15 & 1 & -3.440758 & 0.254598 & 1.502031 \\
\hline 16 & 6 & -5.278560 & -2.372421 & 0.360850 \\
\hline 17 & 1 & -4.652205 & -3.711407 & -1.214965 \\
\hline 18 & 1 & -5.591108 & -0.881308 & 1.889754 \\
\hline 19 & 1 & -6.234819 & -2.864815 & 0.520179 \\
\hline 20 & 6 & 2.535188 & -1.281013 & 0.003142 \\
\hline 21 & 6 & 2.794056 & -2.465165 & -0.728519 \\
\hline 22 & 6 & 3.422829 & -0.962149 & 1.053629 \\
\hline 23 & 6 & 3.890939 & -3.272195 & -0.439560 \\
\hline 24 & 1 & 2.111612 & -2.725948 & -1.529182 \\
\hline 25 & 6 & 4.517327 & -1.783586 & 1.344260 \\
\hline 26 & 1 & 3.234785 & $-\odot .069926$ & 1.636213 \\
\hline 27 & 6 & 4.765154 & -2.941066 & 0.604498 \\
\hline 28 & 1 & 4.063227 & -4.169771 & -1.030121 \\
\hline 29 & 1 & 5.178282 & -1.510980 & 2.164466 \\
\hline 30 & 1 & 5.616280 & -3.577134 & 0.836838 \\
\hline 31 & 6 & -1.401262 & 2.054807 & 0.259432 \\
\hline 32 & 6 & 1.282510 & 1.938494 & -1.197882 \\
\hline 33 & 6 & -0.741658 & 3.150791 & 1.118437 \\
\hline 34 & 1 & 0.417907 & 1.723586 & -1.831372 \\
\hline 35 & 1 & 2.149369 & 1.594086 & -1.780411 \\
\hline 36 & 6 & 1.476153 & 3.453048 & -0.950433 \\
\hline 37 & 6 & $-\odot .542877$ & 4.479151 & 0.374266 \\
\hline 38 & 1 & -1.347539 & 3.329391 & 2.018554 \\
\hline 39 & 1 & ๑. 231411 & 2.798081 & 1.466843 \\
\hline $4 \odot$ & 6 & 0.221628 & 4.355934 & -0.961623 \\
\hline 41 & 1 & 2.023065 & 3.572266 & $-\odot .006837$ \\
\hline 42 & 1 & 2.143205 & 3.822225 & -1.740766 \\
\hline 43 & 1 & -1.514140 & 4.959202 & 0.176144 \\
\hline 44 & 1 & 0.007777 & 5.165932 & 1.034061 \\
\hline 45 & 1 & -0.458684 & 4.009354 & -1.751587 \\
\hline 46 & 1 & 0.524702 & 5.368776 & -1.264686 \\
\hline 47 & 1 & -1.229081 & 2.229014 & -0.804735 \\
\hline 48 & 1 & -2.501397 & 2.144714 & 0.363868 \\
\hline
\end{tabular}

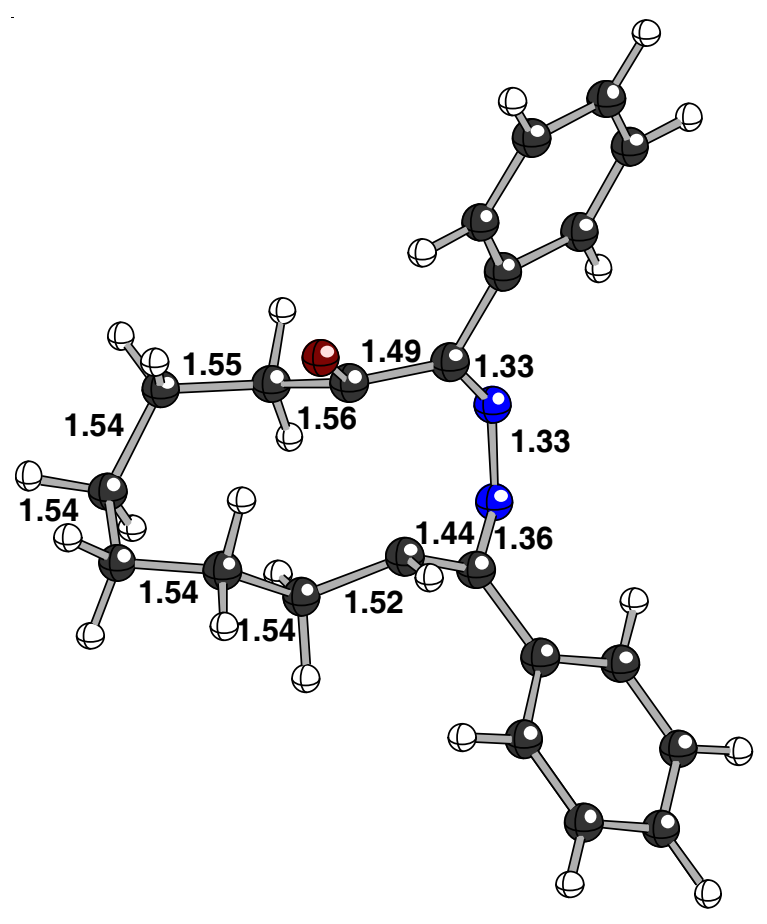




\section{Cyclooctanone Fragmentation TS (Table 2)}

$\mathrm{HF}=-1036.9377968$ hartrees $(-650688.836869968 \mathrm{kcal} / \mathrm{mol})$

Imaginary Frequencies: $1(-352.6731$ 1/cm)

Zero-point correction $=0.397526$ (Hartree/Particle)

Temperature 298.150 Kelvin. Pressure 1.00000 Atm.

Sum of electronic and thermal Free Energies $=-\mathbf{1 0 3 6 . 5 9 0 0 9 0}$ hartrees $(-650470.6473759 \mathrm{kcal} / \mathrm{mol})$

Solvent Single Point: HF = -1037.01594 hartrees $(-650737.8726 \mathrm{kcal} / \mathrm{mol})$

Coordinates (from last standard orientation):

\begin{tabular}{|c|c|c|c|c|}
\hline \multirow{2}{*}{$\begin{array}{l}\text { Center } \\
\text { Number }\end{array}$} & \multirow{2}{*}{$\begin{array}{l}\text { Atomic } \\
\text { Number }\end{array}$} & \multicolumn{3}{|c|}{ Coordinates (Angstroms) } \\
\hline & & $\mathrm{x}$ & $\mathrm{Y}$ & Z \\
\hline 1 & 6 & -0.954018 & 0.746616 & $\odot .185651$ \\
\hline 2 & 6 & -1.629361 & -0.630044 & -0.066837 \\
\hline 3 & 6 & 0.724760 & 0.261356 & -1.174389 \\
\hline 4 & 1 & -0.123997 & $\odot .132917$ & -1.842204 \\
\hline 5 & 6 & 1.153983 & -0.931166 & -0.525605 \\
\hline 6 & 8 & -1.507365 & 1.752146 & -0.336593 \\
\hline 7 & 7 & -1.016477 & -1.757440 & -0.279513 \\
\hline 8 & 7 & 0.343149 & -1.899924 & -0.112993 \\
\hline 9 & 6 & 2.586141 & -1.209971 & -0.160880 \\
\hline 10 & 6 & 2.913895 & -1.667584 & 1.126347 \\
\hline 11 & 6 & 3.621134 & -1.090405 & -1.102411 \\
\hline 12 & 6 & 4.232388 & -1.973504 & 1.468375 \\
\hline 13 & 1 & 2.112480 & -1.792096 & 1.847073 \\
\hline 14 & 6 & 4.940916 & -1.407377 & -0.768576 \\
\hline 15 & 1 & 3.383889 & -0.761089 & -2.109098 \\
\hline 16 & 6 & 5.254501 & -1.843955 & 0.521905 \\
\hline 17 & 1 & 4.462931 & -2.317745 & 2.474030 \\
\hline 18 & 1 & 5.723754 & -1.315492 & -1.517956 \\
\hline 19 & 1 & 6.281643 & -2.084440 & 0.785837 \\
\hline 20 & 6 & -3.117101 & -0.703203 & -0.039526 \\
\hline 21 & 6 & -3.772597 & -1.886309 & -0.450562 \\
\hline 22 & 6 & -3.922462 & 0.364914 & 0.406332 \\
\hline 23 & 6 & -5.159845 & -1.993197 & -0.421667 \\
\hline 24 & 1 & -3.158976 & -2.714931 & -0.786465 \\
\hline 25 & 6 & -5.316321 & 0.252370 & 0.436276 \\
\hline 26 & 1 & -3.451529 & 1.293303 & 0.703751 \\
\hline 27 & 6 & -5.948199 & -0.922347 & 0.023835 \\
\hline 28 & 1 & -5.632716 & -2.917710 & -0.747048 \\
\hline 29 & 1 & -5.909271 & 1.096036 & 0.783141 \\
\hline 30 & 1 & -7.032273 & -1.006106 & 0.047524 \\
\hline 31 & 1 & 0.055319 & -0.112369 & 1.892366 \\
\hline 32 & 6 & -0.269520 & 0.879034 & 1.555555 \\
\hline 33 & 6 & 0.914305 & 1.855848 & 1.673359 \\
\hline 34 & 1 & -1.070935 & 1.197129 & 2.243497 \\
\hline 35 & 6 & 0.704418 & 3.279605 & 1.097097 \\
\hline 36 & 1 & 1.791268 & 1.397039 & 1.204510 \\
\hline 37 & 1 & 1.160332 & 1.931111 & 2.742271 \\
\hline 38 & 6 & 1.486260 & 3.598259 & -0.192318 \\
\hline 39 & 1 & -0.360644 & 3.446171 & ๑.912860 \\
\hline $4 \odot$ & 1 & 1.022316 & 4.015967 & 1.850383 \\
\hline 41 & 6 & 1.663106 & 1.376920 & -1.602698 \\
\hline 42 & 6 & 1.132085 & 2.835318 & -1.487914 \\
\hline 43 & 1 & 1.347200 & 4.671453 & -0.395544 \\
\hline 44 & 1 & 2.563811 & 3.470732 & $\odot .005626$ \\
\hline 45 & 1 & 1.937948 & 1.227574 & -2.663239 \\
\hline 46 & 1 & 2.609894 & 1.321149 & -1.051976 \\
\hline 47 & 1 & ๑.047847 & 2.850081 & -1.634479 \\
\hline 48 & 1 & 1.581124 & 3.408623 & -2.312675 \\
\hline
\end{tabular}

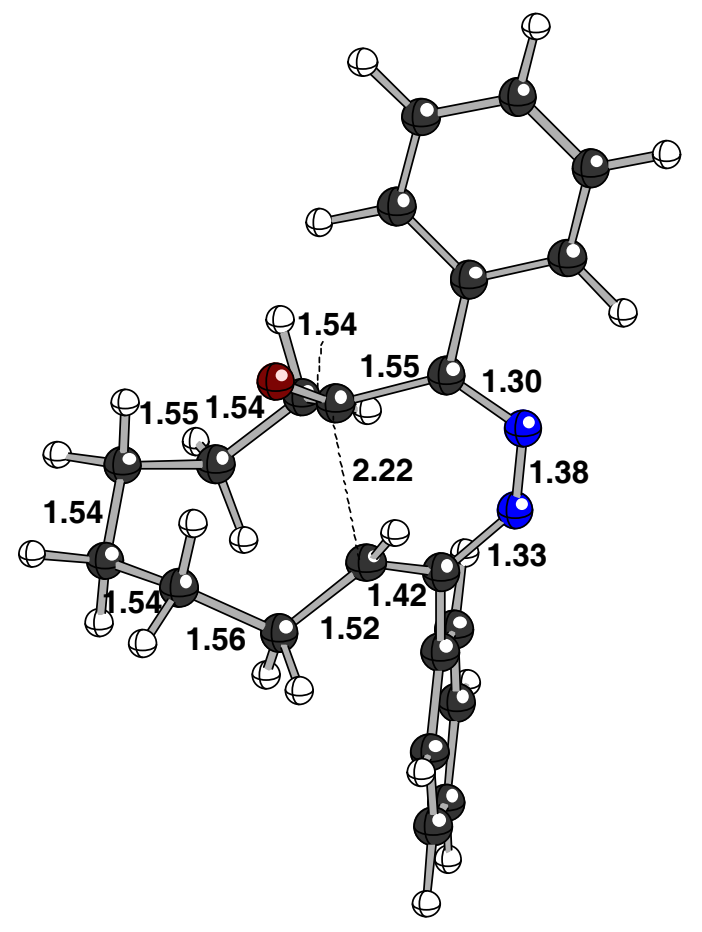




\section{Cyclooctanone Electrocyclic product (Table 2)}

$\mathrm{HF}=-1036.9638777$ hartrees $(-650705.202895527 \mathrm{kcal} / \mathrm{mol})$

Imaginary Frequencies: none found

Zero-point correction $=0.398322$ (Hartree/Particle)

Temperature 298.150 Kelvin. Pressure 1.00000 Atm.

Sum of electronic and thermal Free Energies = -1036.617566 hartrees $(-650487.88884066 \mathrm{kcal} / \mathrm{mol})$

Solvent Single Point: HF = -1037.040177 hartrees $(-650753.0817 \mathrm{kcal} / \mathrm{mol})$

Coordinates (from last standard orientation):

\begin{tabular}{|c|c|c|c|c|}
\hline \multirow{2}{*}{$\begin{array}{l}\text { Center } \\
\text { Number }\end{array}$} & \multirow{2}{*}{$\begin{array}{l}\text { Atomic } \\
\text { Number }\end{array}$} & \multicolumn{3}{|c|}{ Coordinates (Angstroms) } \\
\hline & & $\mathrm{X}$ & $\mathrm{Y}$ & Z \\
\hline & & -------- & ------ & 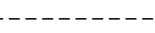 \\
\hline 1 & 6 & 0.007450 & -1.234826 & 0.739440 \\
\hline 2 & 6 & -0.924318 & -0.851055 & -0.342075 \\
\hline 3 & 6 & 2.470013 & 1.254198 & -0.679693 \\
\hline 4 & 1 & 2.746177 & 2.145861 & -0.117093 \\
\hline 5 & 6 & 1.138967 & 0.999224 & -0.822200 \\
\hline 6 & 8 & -0.254909 & -1.162977 & 1.950156 \\
\hline 7 & 7 & $-\odot .490195$ & $-\odot .537558$ & -1.587603 \\
\hline 8 & 7 & 0.622575 & 0.111398 & -1.794508 \\
\hline 9 & 6 & 0.173656 & 1.953449 & -0.169726 \\
\hline 10 & 6 & 0.337370 & 2.360951 & 1.163808 \\
\hline 11 & 6 & -0.873711 & 2.530047 & -0.910805 \\
\hline 12 & 6 & -0.503405 & 3.320244 & 1.734328 \\
\hline 13 & 1 & 1.111895 & 1.895615 & 1.765241 \\
\hline 14 & 6 & -1.715732 & 3.485863 & -0.342927 \\
\hline 15 & 1 & -1.021660 & 2.215189 & -1.938777 \\
\hline 16 & 6 & -1.534032 & 3.891327 & ๑. 984012 \\
\hline 17 & 1 & -0.364277 & 3.604093 & 2.774953 \\
\hline 18 & 1 & -2.519625 & 3.913507 & -0.937612 \\
\hline 19 & 1 & -2.195501 & 4.630504 & 1.429295 \\
\hline 20 & 6 & -2.370456 & -1.117896 & -0.213335 \\
\hline 21 & 6 & -3.222539 & -0.943331 & -1.333943 \\
\hline 22 & 6 & -2.979030 & -1.558142 & ๑.986775 \\
\hline 23 & 6 & -4.584053 & -1.228268 & -1.267630 \\
\hline 24 & 1 & -2.782609 & -0.588425 & -2.259073 \\
\hline 25 & 6 & -4.346961 & -1.831042 & 1.047508 \\
\hline 26 & 1 & -2.368372 & -1.666226 & 1.874242 \\
\hline 27 & 6 & -5.166906 & -1.676010 & -0.074813 \\
\hline 28 & 1 & -5.199731 & -1.088498 & -2.154463 \\
\hline 29 & 1 & -4.775946 & -2.164587 & 1.990663 \\
\hline 30 & 1 & -6.232976 & -1.883410 & -0.019310 \\
\hline 31 & 6 & 3.625146 & ๑. 454834 & -1.227620 \\
\hline 32 & 6 & 1.239800 & -2.044418 & ๑. 311335 \\
\hline 33 & 6 & 4.713445 & ๑. 168878 & -0.141902 \\
\hline 34 & 1 & 1. 383952 & -1.958439 & -0.766287 \\
\hline 35 & 1 & ๑. 961040 & -3.091183 & ๑.512505 \\
\hline 36 & 6 & 2.555441 & -1.711549 & 1.037934 \\
\hline 37 & 6 & 5.006908 & -1.314674 & 0.194755 \\
\hline 38 & 1 & 5.664420 & 0.635089 & -0.434978 \\
\hline 39 & 1 & 4.412671 & 0.679740 & 0.780434 \\
\hline 40 & 6 & 3.781627 & -2.249202 & 0. 261232 \\
\hline 41 & 1 & 2.621223 & -0.625074 & 1.132102 \\
\hline 42 & 1 & 2.532441 & -2.105050 & 2.060135 \\
\hline 43 & 1 & 5.700624 & -1.729331 & -0.551665 \\
\hline 44 & 1 & 5.549575 & -1.338565 & 1.151612 \\
\hline 45 & 1 & 3.471285 & -2.464688 & -0.768586 \\
\hline 46 & 1 & 4.102726 & -3.216579 & 0.673117 \\
\hline 47 & 1 & 3.247559 & -0.470317 & -1.671938 \\
\hline 48 & 1 & 4.095632 & 1. 006091 & -2.057714 \\
\hline
\end{tabular}

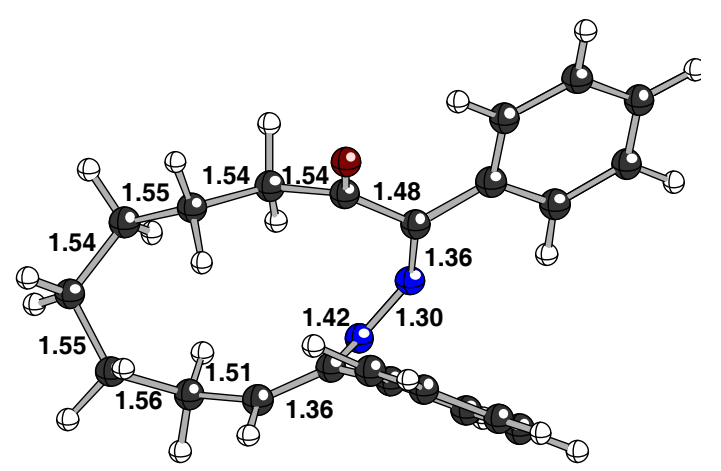




\section{Cyclooctanone Fragmentation product (Table 2)}

$\mathrm{HF}=-1036.9554973$ hartrees $(-650699.944110723 \mathrm{kcal} / \mathrm{mol})$

Imaginary Frequencies: none found

Zero-point correction $=0.398127$ (Hartree/Particle)

Temperature 298.150 Kelvin. Pressure 1.00000 Atm.

Sum of electronic and thermal Free Energies $=-\mathbf{1 0 3 6 . 6 0 9 3 1 6 ~ h a r t r e e s ~}(-650482.71188316 \mathrm{kcal} / \mathrm{mol})$

Solvent Single Point: HF = -1037.032396 hartrees $(-650748.199 \mathrm{kcal} / \mathrm{mol})$

Coordinates (from last standard orientation):

\begin{tabular}{|c|c|c|c|c|}
\hline \multirow{2}{*}{$\begin{array}{l}\text { Center } \\
\text { Number }\end{array}$} & \multirow{2}{*}{$\begin{array}{l}\text { Atomic } \\
\text { Number }\end{array}$} & \multicolumn{3}{|c|}{ Coordinates (Angstroms) } \\
\hline & & $\mathrm{x}$ & $\mathrm{Y}$ & Z \\
\hline 1 & 6 & 1.303952 & $\odot .934210$ & -0.729204 \\
\hline 2 & 6 & 1.780214 & -0.359457 & -0.220793 \\
\hline 3 & 6 & -0.934703 & 0.343452 & 1.302833 \\
\hline 4 & 1 & 0.103216 & 0.603247 & 1.494704 \\
\hline 5 & 6 & -1.166699 & $-\odot .730782$ & 0.495842 \\
\hline 6 & 8 & 1.879832 & 2.006274 & -0.466782 \\
\hline 7 & 7 & 1.053693 & -1.498788 & -0.110596 \\
\hline 8 & 7 & $-\odot .225572$ & -1.672102 & 0.016581 \\
\hline 9 & 6 & -2.553637 & -1.199426 & 0.166744 \\
\hline 10 & 6 & -2.872009 & -1.571992 & -1.151238 \\
\hline 11 & 6 & -3.553701 & -1.338879 & 1.143584 \\
\hline 12 & 6 & -4.150068 & -2.018742 & -1.489390 \\
\hline 13 & 1 & -2.095319 & -1.519683 & -1.907266 \\
\hline 14 & 6 & -4.834188 & -1.792752 & $\odot .812185$ \\
\hline 15 & 1 & -3.316376 & -1.112503 & 2.178274 \\
\hline 16 & 6 & -5.142543 & -2.127000 & $-\odot .508958$ \\
\hline 17 & 1 & -4.370133 & -2.291668 & -2.518859 \\
\hline 18 & 1 & -5.586981 & -1.895672 & 1.590475 \\
\hline 19 & 1 & -6.137284 & -2.480466 & $-\odot .769354$ \\
\hline 20 & 6 & 3.237093 & -0.573230 & $-\odot . \odot \odot 2468$ \\
\hline 21 & 6 & 3.701297 & -1.839976 & 0.438191 \\
\hline 22 & 6 & 4.228564 & 0.413825 & $-\odot .224286$ \\
\hline 23 & 6 & 5.056970 & -2.107241 & 0.614043 \\
\hline 24 & 1 & 2.964429 & -2.611366 & 0.627848 \\
\hline 25 & 6 & 5.586044 & 0.140485 & $-\odot .040185$ \\
\hline 26 & 1 & 3.925785 & 1.406644 & -0.528580 \\
\hline 27 & 6 & 6.022243 & -1.120074 & 0.378369 \\
\hline 28 & 1 & 5.362089 & -3.097236 & 0.949039 \\
\hline 29 & 1 & 6.310269 & 0.932239 & -0.224245 \\
\hline 30 & 1 & 7.079828 & -1.324746 & $\odot .528168$ \\
\hline 31 & 1 & $-\odot .077066$ & $-\odot .033307$ & -2.103593 \\
\hline 32 & 6 & 0.213831 & 0.981341 & -1.813753 \\
\hline 33 & 6 & -1.074329 & 1.811242 & -1.554680 \\
\hline 34 & 1 & 0.745749 & 1.424211 & -2.668062 \\
\hline 35 & 6 & -0.918623 & 3.182151 & -0.848013 \\
\hline 36 & 1 & -1.766164 & 1.196018 & $-\odot .982077$ \\
\hline 37 & 1 & -1.554367 & 1.959024 & -2.532183 \\
\hline 38 & 6 & -1.910895 & 3.435869 & 0.307865 \\
\hline 39 & 1 & 0.101238 & 3.292618 & -0.470687 \\
\hline 40 & 1 & -1.053204 & 3.981993 & -1.590131 \\
\hline 41 & 6 & -1.928236 & 1.305976 & 1.901657 \\
\hline 42 & 6 & -1.587812 & 2.804266 & 1.684469 \\
\hline 43 & 1 & -1.956691 & 4.523511 & 0.466676 \\
\hline 44 & 1 & -2.926901 & 3.137010 & $\odot .004098$ \\
\hline 45 & 1 & -1.983306 & 1.154094 & 2.994674 \\
\hline 46 & 1 & $-2.94 \odot 282$ & 1.120887 & 1.524792 \\
\hline 47 & 1 & $-\odot .525960$ & 2.970548 & 1.913356 \\
\hline 48 & 1 & -2.154654 & 3.371047 & 2.438412 \\
\hline
\end{tabular}

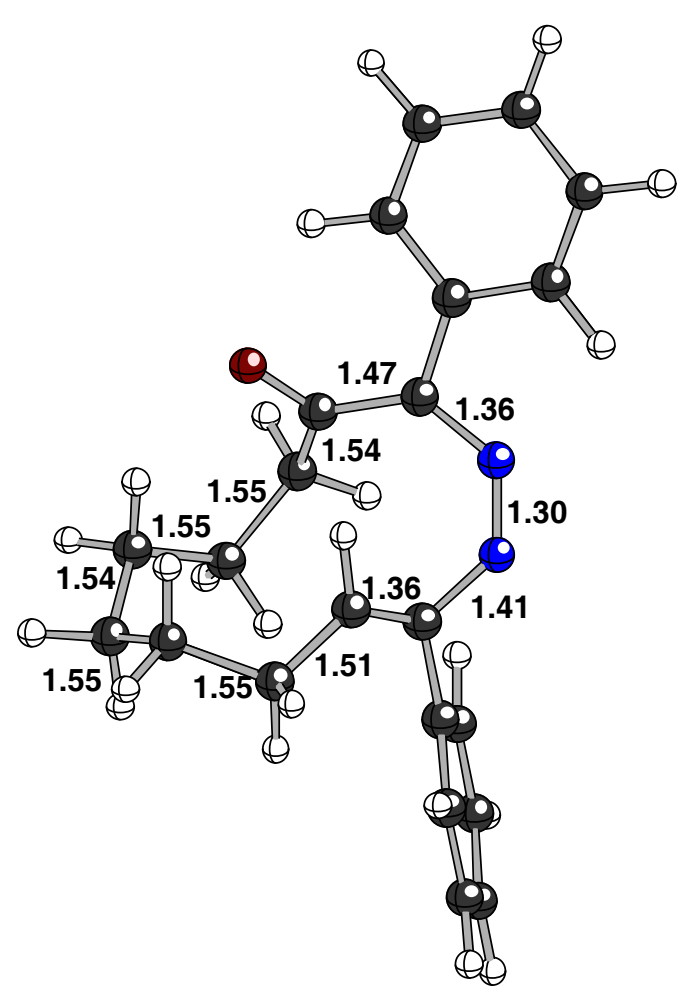




\section{2 ( $n=1$, pyridazine figure and scheme in SI and footnote 26)}

$\mathrm{HF}=-879.702823$ hartrees $(-552022.31846073 \mathrm{kcal} / \mathrm{mol})$

Imaginary Frequencies: none found

Zero-point correction $=0.283797$ (Hartree/Particle)

Temperature 298.150 Kelvin. Pressure 1.00000 Atm.

Sum of electronic and thermal Free Energies = $\mathbf{- 8 7 9 . 4 6 4 3 2 3 ~ h a r t r e e s ~}(-551872.65732573 \mathrm{kcal} / \mathrm{mol})$

Solvent Single Point: HF = -879.7870874 hartrees $(-552075.1952 \mathrm{kcal} / \mathrm{mol})$

Coordinates (from last standard orientation):

\begin{tabular}{|c|c|c|c|c|}
\hline \multirow{2}{*}{$\begin{array}{l}\text { Center } \\
\text { Number }\end{array}$} & \multirow{2}{*}{$\begin{array}{l}\text { Atomic } \\
\text { Number }\end{array}$} & \multicolumn{3}{|c|}{ Coordinates (Angstroms) } \\
\hline & & $\mathrm{x}$ & $\mathrm{Y}$ & Z \\
\hline 1 & 6 & 0.778438 & 2.243697 & -0.760265 \\
\hline 2 & 1 & 1.220673 & 1.862059 & -1.685194 \\
\hline 3 & 1 & 1.269746 & 3.179533 & $-\odot .471522$ \\
\hline 4 & 6 & -0.793740 & 2.269441 & -0.807030 \\
\hline 5 & 1 & -1.261429 & 2.178452 & -1.796909 \\
\hline 6 & 1 & -1.203524 & 3.166158 & -0.318651 \\
\hline 7 & 6 & 0.694286 & 1.156518 & $\odot .356719$ \\
\hline 8 & 6 & 1.312537 & $-\odot .198937$ & 0.311910 \\
\hline 9 & 6 & -0.755339 & 1.022175 & 0.054839 \\
\hline 10 & 6 & -1.433734 & -0.141249 & 0.292803 \\
\hline 11 & 8 & 0.987240 & 1.865807 & 1.633021 \\
\hline 12 & 7 & 0.575026 & -1.280823 & 0.592596 \\
\hline 13 & 7 & -0.750607 & -1.280093 & 0.698790 \\
\hline 14 & 6 & -2.887501 & -0.321866 & 0.063946 \\
\hline 15 & 6 & -3.448671 & -1.611613 & -0.021565 \\
\hline 16 & 6 & -3.754822 & 0.780503 & -0.066643 \\
\hline 17 & 6 & -4.816418 & -1.787868 & -0.235030 \\
\hline 18 & 1 & -2.779753 & -2.458089 & ๑.088473 \\
\hline 19 & 6 & -5.121847 & 0.603862 & $-\odot .291236$ \\
\hline 20 & 1 & -3.351735 & 1.784155 & 0.024345 \\
\hline 21 & 6 & -5.664104 & -0.682877 & -0.377368 \\
\hline 22 & 1 & -5.224376 & -2.794830 & -0.295884 \\
\hline 23 & 1 & -5.767033 & 1.474340 & $-\odot .389770$ \\
\hline 24 & 1 & -6.729019 & $-\odot .8219 \odot 4$ & -0.548723 \\
\hline 25 & 6 & 2.738881 & -0.412345 & ๑.037578 \\
\hline 26 & 6 & 3.270515 & -1.712389 & -0.145049 \\
\hline 27 & 6 & 3.643109 & 0.670793 & -0.059103 \\
\hline 28 & 6 & 4.623064 & -1.911828 & $-\odot .4 \odot 8527$ \\
\hline 29 & 1 & 2.587808 & -2.552047 & -0.070859 \\
\hline 30 & 6 & 4.996405 & 0.466801 & -0.335776 \\
\hline 31 & 1 & 3.281071 & 1.678591 & 0.113210 \\
\hline 32 & 6 & 5.503531 & -0.824872 & -0.513287 \\
\hline 33 & 1 & 4.996945 & -2.925507 & -0.542172 \\
\hline 34 & 1 & 5.662083 & 1.325460 & $-\odot .401139$ \\
\hline 35 & 1 & 6.558113 & -0.983030 & -0.726137 \\
\hline 36 & 1 & 0.764030 & 1.238935 & 2.335556 \\
\hline
\end{tabular}

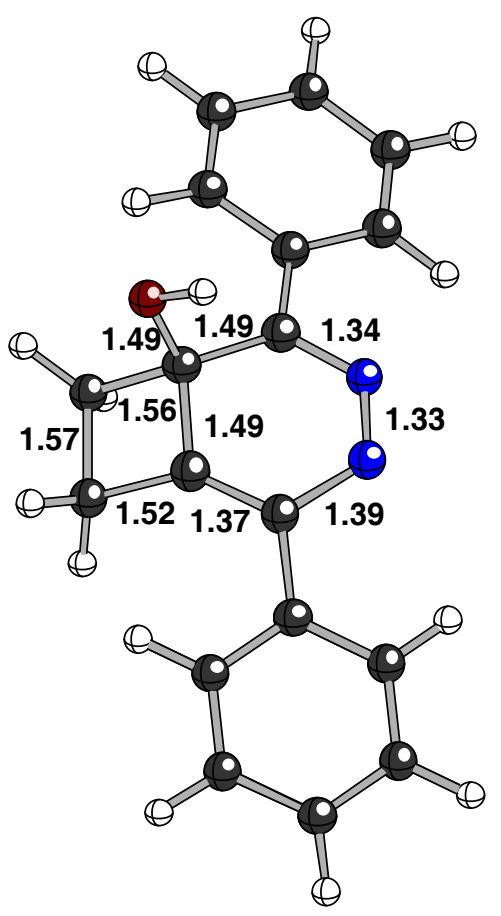




\section{2 ( $n=1$, pyridazine figure and scheme in SI and footnote 26)}

$\mathrm{HF}=-879.7882136$ hartrees $(-552075.901916136 \mathrm{kcal} / \mathrm{mol})$

Imaginary Frequencies: none found

Zero-point correction $=0.282706$ (Hartree/Particle)

Temperature 298.150 Kelvin. Pressure 1.00000 Atm.

Sum of electronic and thermal Free Energies = -879.551162 hartrees $(-551927.14966662 \mathrm{kcal} / \mathrm{mol})$

\begin{tabular}{|c|c|c|c|c|}
\hline \multirow{2}{*}{$\begin{array}{l}\text { Center } \\
\text { Number }\end{array}$} & \multirow{2}{*}{$\begin{array}{l}\text { Atomic } \\
\text { Number }\end{array}$} & \multicolumn{3}{|c|}{ Coordinates (Angstroms) } \\
\hline & & $\mathrm{x}$ & $\mathrm{Y}$ & Z \\
\hline 1 & 6 & 0.776666 & 2.167289 & $-\odot .858072$ \\
\hline 2 & 1 & 1.222934 & 1.729549 & -1.760642 \\
\hline 3 & 1 & 1.266703 & 3.123660 & -0.628796 \\
\hline 4 & 6 & -0.796568 & 2.184486 & $-\odot .916152$ \\
\hline 5 & 1 & -1.257411 & 2.026447 & -1.899847 \\
\hline 6 & 1 & -1.218102 & 3.105150 & -0.488686 \\
\hline 7 & 6 & 0.690573 & 1.152992 & 0.323413 \\
\hline 8 & 6 & 1.318669 & $-\odot .201419$ & $\odot .353128$ \\
\hline 9 & 6 & -0.757373 & 0.994203 & 0.024283 \\
\hline 10 & 6 & -1.438440 & -0.148553 & 0.334139 \\
\hline 11 & 8 & 0.972370 & 1.915097 & 1.565965 \\
\hline 12 & 7 & $\odot .591363$ & -1.257800 & 0.720884 \\
\hline 13 & 7 & -0.752541 & -1.251777 & 0.834000 \\
\hline 14 & 6 & -2.892731 & $-\odot .332977$ & 0.089019 \\
\hline 15 & 6 & -3.446029 & -1.609151 & -0.129725 \\
\hline 16 & 6 & -3.763084 & 0.774855 & 0.064957 \\
\hline 17 & 6 & -4.813778 & -1.771120 & -0.366053 \\
\hline 18 & 1 & -2.787861 & -2.472156 & -0.116929 \\
\hline 19 & 6 & -5.129269 & 0.615654 & -0.179328 \\
\hline 20 & 1 & -3.366061 & 1.767751 & ๑. 255938 \\
\hline 21 & 6 & -5.663574 & -0.659793 & -0.395441 \\
\hline 22 & 1 & -5.216151 & -2.768009 & -0.534583 \\
\hline 23 & 1 & -5.779220 & 1.488138 & -0.190592 \\
\hline 24 & 1 & -6.727684 & -0.785622 & $-\odot .581991$ \\
\hline 25 & 6 & 2.749169 & -0.415580 & ๑.061233 \\
\hline 26 & 6 & 3.284760 & -1.707546 & -0.146308 \\
\hline 27 & 6 & 3.640962 & 0.676256 & -0.033034 \\
\hline 28 & 6 & 4.639534 & -1.896643 & -0.419135 \\
\hline 29 & 1 & 2.617864 & -2.562566 & -0.094398 \\
\hline 30 & 6 & 4.995355 & 0.486634 & -0.318622 \\
\hline 31 & 1 & 3.271389 & 1.681449 & 0.139693 \\
\hline 32 & 6 & 5.508186 & -0.800830 & -0.511334 \\
\hline 33 & 1 & 5.019231 & -2.905009 & -0.573030 \\
\hline 34 & 1 & 5.654413 & 1.350323 & -0.382160 \\
\hline 35 & 1 & 6.562702 & -0.948734 & -0.732073 \\
\hline 36 & 1 & 0.668372 & 1.363343 & 2.314382 \\
\hline
\end{tabular}

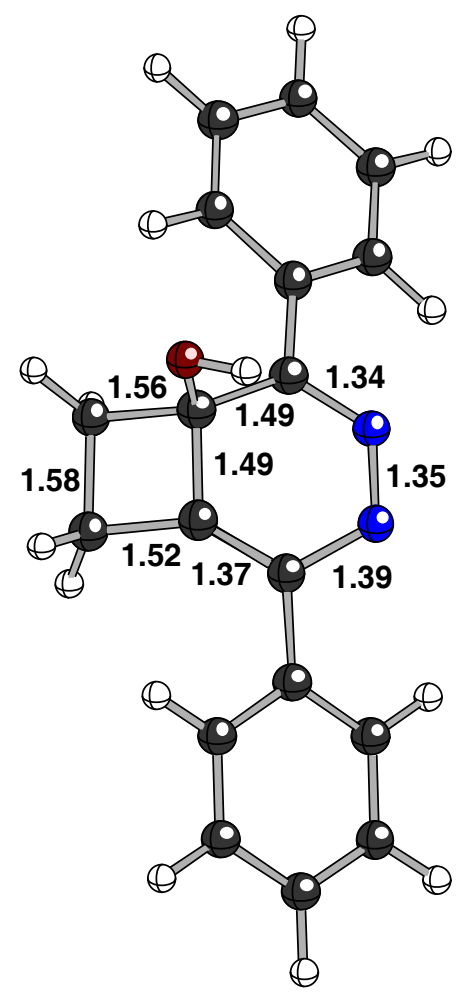


TS12 $\rightarrow$ pyridazines ( $n=1$, pyridazine figure and scheme in SI and footnote 26)

$\mathrm{HF}=-879.6806217$ hartrees $(-552008.386922967 \mathrm{kcal} / \mathrm{mol})$

Imaginary Frequencies: 1 (-123.8210 1/cm)

Zero-point correction $=0.281583$ (Hartree/Particle)

Temperature 298.150 Kelvin. Pressure 1.00000 Atm.

Sum of electronic and thermal Free Energies = -879.444764 hartrees $(-551860.38385764 \mathrm{kcal} / \mathrm{mol})$

Solvent Single Point: HF = -879.787017 hartrees $(-552075.151 \mathrm{kcal} / \mathrm{mol})$

Coordinates (from last standard orientation):

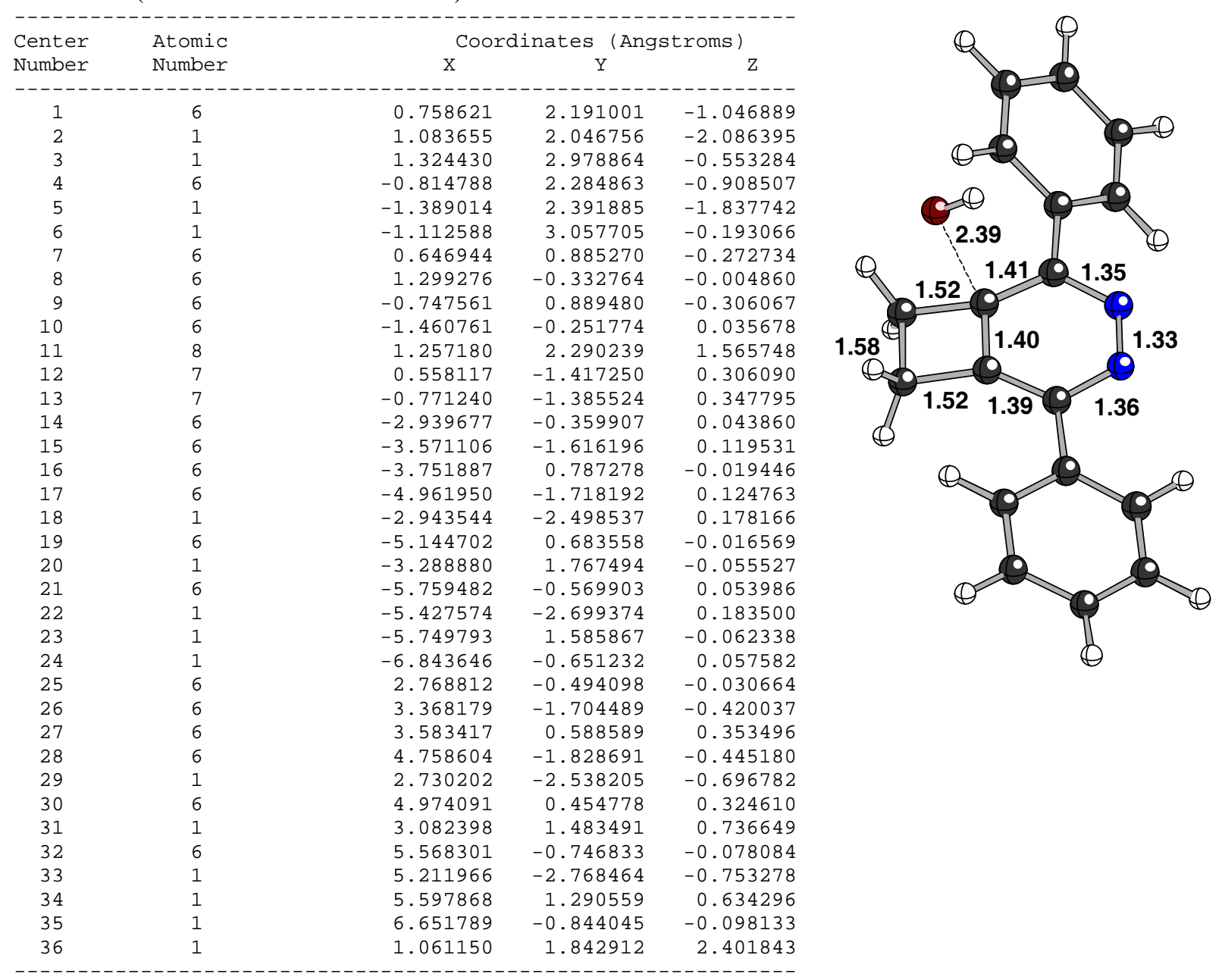


TS12 $\rightarrow$ pyridazines ( $n=1$, pyridazine figure and scheme in SI and footnote 26)

(solvent optimization, methanol, ua0 radii)

$\mathrm{HF}=-879.7738438$ hartrees $(-552066.884722938 \mathrm{kcal} / \mathrm{mol})$

Imaginary Frequencies: 1 (-267.4212 1/cm)

Zero-point correction $=0.280250$ (Hartree/Particle)

Temperature 298.150 Kelvin. Pressure 1.00000 Atm.

Sum of electronic and thermal Free Energies = -879.540020 hartrees $(-551920.1579502 \mathrm{kcal} / \mathrm{mol})$

Coordinates (from last standard orientation):

\begin{tabular}{|c|c|c|c|c|}
\hline \multirow{2}{*}{$\begin{array}{l}\text { Center } \\
\text { Number }\end{array}$} & \multirow{2}{*}{$\begin{array}{l}\text { Atomic } \\
\text { Number }\end{array}$} & \multicolumn{3}{|c|}{ Coordinates (Angstroms) } \\
\hline & & $\mathrm{x}$ & $\mathrm{Y}$ & Z \\
\hline 1 & 6 & $\odot .767757$ & 2.224893 & -0.919044 \\
\hline 2 & 1 & 1.203819 & 1.996997 & -1.898772 \\
\hline 3 & 1 & 1.255968 & 3.099389 & -0.487436 \\
\hline 4 & 6 & -0.811678 & 2.245205 & $-\odot .939752$ \\
\hline 5 & 1 & -1.281781 & 2.208023 & -1.929347 \\
\hline 6 & 1 & -1.240192 & 3.082064 & -0.377001 \\
\hline 7 & 6 & 0.659938 & $\odot .997463$ & -0.014009 \\
\hline 8 & 6 & 1.324911 & -0.243883 & 0.196535 \\
\hline 9 & 6 & -0.739342 & 0.929083 & -0.181773 \\
\hline 10 & 6 & -1.443553 & $-\odot .208980$ & 0.168234 \\
\hline 11 & 8 & 0.973444 & 2.041501 & 1.759209 \\
\hline 12 & 7 & $\odot .592312$ & -1.308897 & 0.570896 \\
\hline 13 & 7 & -0.745709 & -1.297656 & 0.618889 \\
\hline 14 & 6 & -2.914605 & -0.358785 & 0.046224 \\
\hline 15 & 6 & -3.505613 & -1.623506 & -0.135859 \\
\hline 16 & 6 & -3.755417 & 0.769017 & 0.103487 \\
\hline 17 & 6 & -4.891168 & -1.754788 & -0.255117 \\
\hline 18 & 1 & -2.867920 & -2.501825 & -0.189107 \\
\hline 19 & 6 & -5.141313 & 0.637925 & -0.018267 \\
\hline 20 & 1 & -3.326348 & 1.754232 & 0.264393 \\
\hline 21 & 6 & -5.716135 & $-\odot .625078$ & -0.198237 \\
\hline 22 & 1 & -5.328185 & -2.742211 & -0.399389 \\
\hline 23 & 1 & -5.772834 & 1.523828 & 0.034928 \\
\hline 24 & 1 & -6.796246 & $-\odot .728215$ & -0.292729 \\
\hline 25 & 6 & 2.782194 & $-\odot .436274$ & 0.028544 \\
\hline 26 & 6 & 3.339248 & -1.718232 & -0.153892 \\
\hline 27 & 6 & 3.650453 & 0.673046 & 0.021712 \\
\hline 28 & 6 & 4.714537 & -1.883432 & -0.328571 \\
\hline 29 & 1 & 2.682161 & -2.583799 & -0.163494 \\
\hline 30 & 6 & 5.026225 & 0.507012 & -0.158935 \\
\hline 31 & 1 & 3.243092 & 1.663894 & 0.194041 \\
\hline 32 & 6 & 5.566848 & -0.771711 & -0.334565 \\
\hline 33 & 1 & 5.122652 & -2.883699 & -0.469615 \\
\hline 34 & 1 & 5.678441 & 1.379590 & -0.154401 \\
\hline 35 & 1 & 6.639000 & -0.901555 & -0.475167 \\
\hline 36 & 1 & $\odot .594881$ & 1.393286 & 2.380626 \\
\hline
\end{tabular}

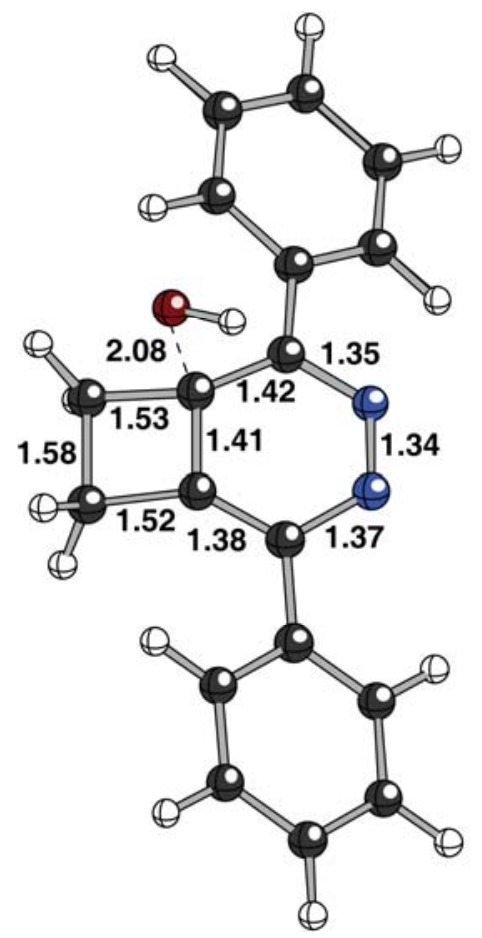




\section{2 ( $n=2$, pyridazine figure and scheme in SI and footnote 26)}

$\mathrm{HF}=-919.0525897$ hartrees $(-576714.690562647 \mathrm{kcal} / \mathrm{mol})$

Imaginary Frequencies: none found

Zero-point correction $=0.313917$ (Hartree/Particle)

Temperature 298.150 Kelvin. Pressure 1.00000 Atm.

Sum of electronic and thermal Free Energies = -918.784548 hartrees $\mathbf{( - 5 7 6 5 4 6 . 4 9 1 7 1 5 4 8 ~} \mathbf{k c a l} / \mathrm{mol})$

Solvent Single Point: HF = -919.1356715 hartrees $(-576766.8252 \mathrm{kcal} / \mathrm{mol})$

Coordinates (from last standard orientation):

\begin{tabular}{|c|c|c|c|c|}
\hline \multirow{2}{*}{$\begin{array}{l}\text { Center } \\
\text { Number }\end{array}$} & \multirow{2}{*}{$\begin{array}{l}\text { Atomic } \\
\text { Number }\end{array}$} & \multicolumn{3}{|c|}{ Coordinates (Angstroms) } \\
\hline & & $\mathrm{x}$ & $\mathrm{Y}$ & Z \\
\hline 1 & 6 & 0.687402 & 1.025489 & 0.314282 \\
\hline 2 & 6 & 1.306673 & -0.336744 & 0.212841 \\
\hline 3 & 6 & -0.772040 & 0.905392 & $\odot .011233$ \\
\hline 4 & 1 & 0.551479 & 0.918201 & 2.304755 \\
\hline 5 & 6 & -1.410898 & $-\odot .297684$ & 0.195713 \\
\hline 6 & 8 & 0.892854 & 1.594269 & 1.702521 \\
\hline 7 & 7 & $\odot .590412$ & -1.454329 & 0.346237 \\
\hline 8 & 7 & -0.733809 & -1.478209 & 0.433930 \\
\hline 9 & 6 & -2.885677 & -0.467354 & 0.091208 \\
\hline 10 & 6 & -3.426962 & -1.681906 & $-\odot .374389$ \\
\hline 11 & 6 & -3.785066 & 0.543988 & $\odot .476679$ \\
\hline 12 & 6 & -4.806786 & -1.867101 & -0.469358 \\
\hline 13 & 1 & -2.734382 & -2.471204 & -0.645666 \\
\hline 14 & 6 & -5.167326 & $\odot .361341$ & $\odot .379469$ \\
\hline 15 & 1 & -3.395116 & 1.473990 & $\odot .877876$ \\
\hline 16 & 6 & -5.688394 & -0.844903 & $-\odot .098197$ \\
\hline 17 & 1 & -5.197897 & -2.813792 & -0.836352 \\
\hline 18 & 1 & -5.838178 & 1.160160 & 0.688440 \\
\hline 19 & 1 & -6.763740 & -0.989062 & -0.173413 \\
\hline 20 & 6 & 2.762828 & -0.531473 & ๑.067387 \\
\hline 21 & 6 & 3.281430 & -1.760273 & $-\odot .405746$ \\
\hline 22 & 6 & 3.699451 & 0.472012 & ๑.403989 \\
\hline 23 & 6 & 4.652088 & -1.968015 & $-\odot .541556$ \\
\hline 24 & 1 & 2.574148 & -2.543624 & $-\odot .656251$ \\
\hline 25 & 6 & 5.073272 & 0.264215 & $\odot .258147$ \\
\hline 26 & 1 & 3.342035 & 1.406942 & 0.820845 \\
\hline 27 & 6 & 5.565848 & -0.955180 & $-\odot .21759 \odot$ \\
\hline 28 & 1 & 5.013388 & -2.925782 & -0.911895 \\
\hline 29 & 1 & 5.764126 & 1.058943 & 0.533538 \\
\hline 30 & 1 & 6.635564 & -1.116220 & -0.329163 \\
\hline 31 & 6 & -1.256347 & 2.188908 & -0.617868 \\
\hline 32 & 1 & -1.467184 & 2.966284 & 0.135953 \\
\hline 33 & 1 & -2.160974 & 2.068662 & -1.223261 \\
\hline 34 & 6 & -0.026814 & 2.613329 & -1.453340 \\
\hline 35 & 1 & -0.045642 & 2.077011 & -2.410522 \\
\hline 36 & 1 & -0.010026 & 3.688028 & -1.676563 \\
\hline 37 & 6 & 1.198077 & 2.167024 & -0.619829 \\
\hline 38 & 1 & 2.021724 & 1.836994 & -1.257843 \\
\hline 39 & 1 & 1.567058 & 2.991703 & $-\odot .0 \odot \odot 253$ \\
\hline
\end{tabular}

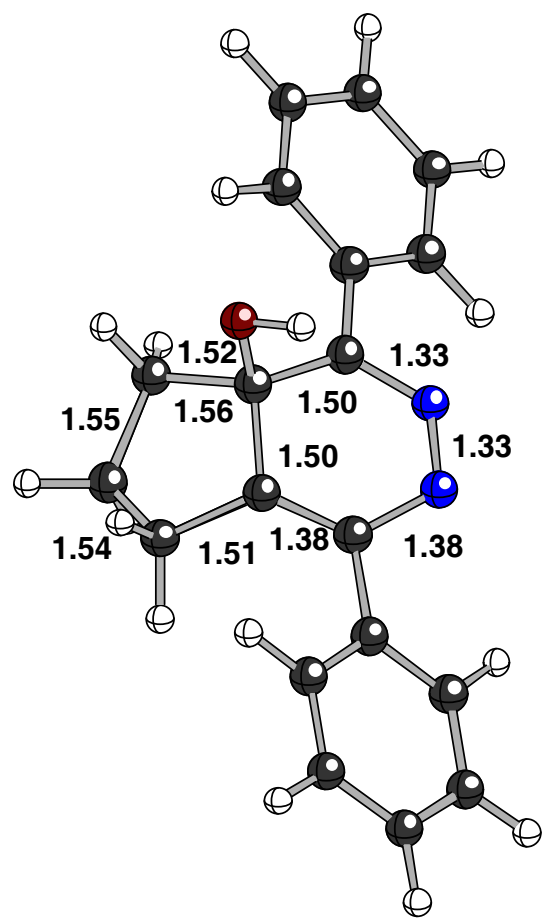




\section{2 ( $n=2$, pyridazine figure and scheme in SI and footnote 26)}

\section{(solvent optimization, methanol, uaks radii)}

$\mathrm{HF}=-919.1369028$ hartrees $(-576767.597876028 \mathrm{kcal} / \mathrm{mol})$

Imaginary Frequencies: none found

Zero-point correction $=0.313139$ (Hartree/Particle)

Temperature 298.150 Kelvin. Pressure 1.00000 Atm.

Sum of electronic and thermal Free Energies $=\mathbf{- 9 1 8 . 8 7 0 0 4 1}$ hartrees $(-576600.13942791 \mathrm{kcal} / \mathrm{mol})$

Coordinates (from last standard orientation):

\begin{tabular}{|c|c|c|c|c|}
\hline \multirow{2}{*}{$\begin{array}{l}\text { Center } \\
\text { Number }\end{array}$} & \multirow{2}{*}{$\begin{array}{l}\text { Atomic } \\
\text { Number }\end{array}$} & \multicolumn{3}{|c|}{ Coordinates (Angstroms) } \\
\hline & & $\mathrm{x}$ & $\mathrm{Y}$ & Z \\
\hline 1 & 6 & 0.683955 & 1.018285 & 0.311575 \\
\hline 2 & 6 & 1.313286 & -0.345182 & 0.268596 \\
\hline 3 & 6 & -0.774430 & 0.876308 & 0.007539 \\
\hline 4 & 1 & 0.509808 & 1.003079 & 2.323306 \\
\hline 5 & 6 & -1.414834 & -0.311875 & $\odot .250940$ \\
\hline 6 & 8 & 0.882541 & 1.630212 & 1.673531 \\
\hline 7 & 7 & $\odot .606907$ & -1.448945 & 0.496450 \\
\hline 8 & 7 & -0.734331 & -1.470987 & 0.587451 \\
\hline 9 & 6 & -2.890427 & -0.477164 & 0.114514 \\
\hline 10 & 6 & -3.438292 & -1.638970 & -0.461278 \\
\hline 11 & 6 & -3.779014 & 0.514708 & 0.569005 \\
\hline 12 & 6 & -4.821047 & -1.800308 & -0.584647 \\
\hline 13 & 1 & -2.766476 & -2.415636 & -0.814735 \\
\hline 14 & 6 & -5.162888 & 0.356696 & 0.447030 \\
\hline 15 & 1 & -3.382154 & 1.411108 & 1.037617 \\
\hline 16 & 6 & -5.691567 & -0.802038 & -0.131938 \\
\hline 17 & 1 & -5.219499 & -2.705331 & -1.038564 \\
\hline 18 & 1 & -5.828126 & 1.136494 & ๑.811796 \\
\hline 19 & 1 & -6.767838 & -0.926904 & -0.226764 \\
\hline 20 & 6 & 2.773556 & -0.530889 & 0.087917 \\
\hline 21 & 6 & 3.291638 & -1.731558 & -0.446315 \\
\hline 22 & 6 & 3.702330 & 0.470520 & 0.445827 \\
\hline 23 & 6 & 4.664921 & -1.926678 & -0.603194 \\
\hline 24 & 1 & 2.597451 & -2.511759 & -0.743775 \\
\hline 25 & 6 & 5.076978 & 0.279448 & $\odot .280255$ \\
\hline 26 & 1 & 3.342464 & 1.396757 & ๑.880827 \\
\hline 27 & 6 & 5.570709 & -0.920685 & -0.243223 \\
\hline 28 & 1 & 5.029396 & -2.863291 & -1.020716 \\
\hline 29 & 1 & 5.765489 & 1.070026 & 0.572144 \\
\hline 30 & 1 & 6.640453 & -1.069264 & $-\odot .370608$ \\
\hline 31 & 6 & -1.262020 & 2.126700 & -0.684131 \\
\hline 32 & 1 & -1.479437 & 2.932147 & 0.035925 \\
\hline 33 & 1 & -2.166261 & 1.976035 & -1.282603 \\
\hline 34 & 6 & -0.030559 & 2.516989 & -1.533112 \\
\hline 35 & 1 & -0.041520 & 1.940275 & -2.467116 \\
\hline 36 & 1 & -0.016477 & 3.579913 & -1.802211 \\
\hline 37 & 6 & 1.192987 & 2.112926 & -0.674976 \\
\hline 38 & 1 & 2.018611 & 1.753685 & -1.294504 \\
\hline 39 & 1 & 1.564067 & 2.967102 & $-\odot .097882$ \\
\hline
\end{tabular}

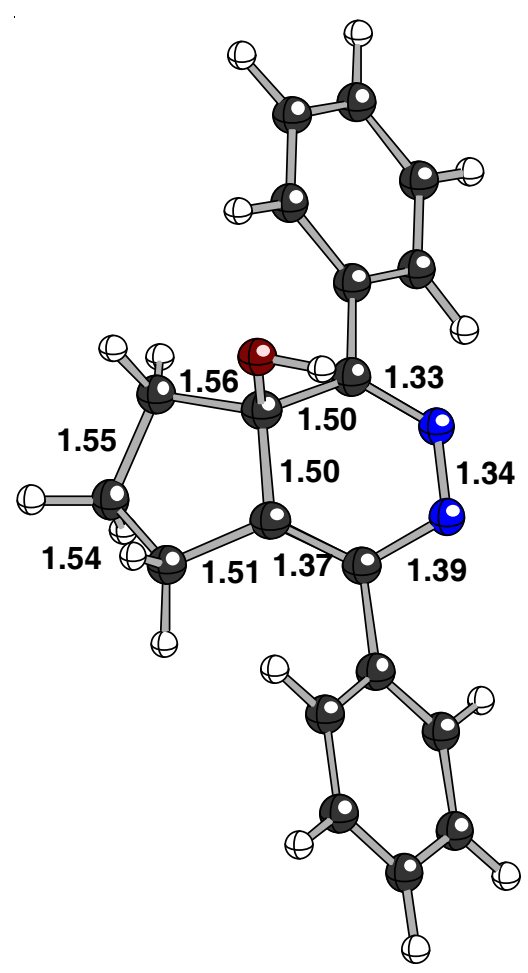


TS12 $\rightarrow$ pyridazines ( $n=2$, pyridazine and scheme figure in SI and footnote 26)

$\mathrm{HF}=-919.0383184$ hartrees $(-576705.735179184 \mathrm{kcal} / \mathrm{mol})$

Imaginary Frequencies: 1 (-162.3356 1/cm)

Zero-point correction $=0.311640$ (Hartree/Particle)

Temperature 298.150 Kelvin. Pressure 1.00000 Atm.

Sum of electronic and thermal Free Energies = -918.773146 hartrees $(-576539.33684646 \mathrm{kcal} / \mathrm{mol})$

Solvent Single Point: HF = -919.1436739 hartrees $(-576771.8468 \mathrm{kcal} / \mathrm{mol})$

Coordinates (from last standard orientation):

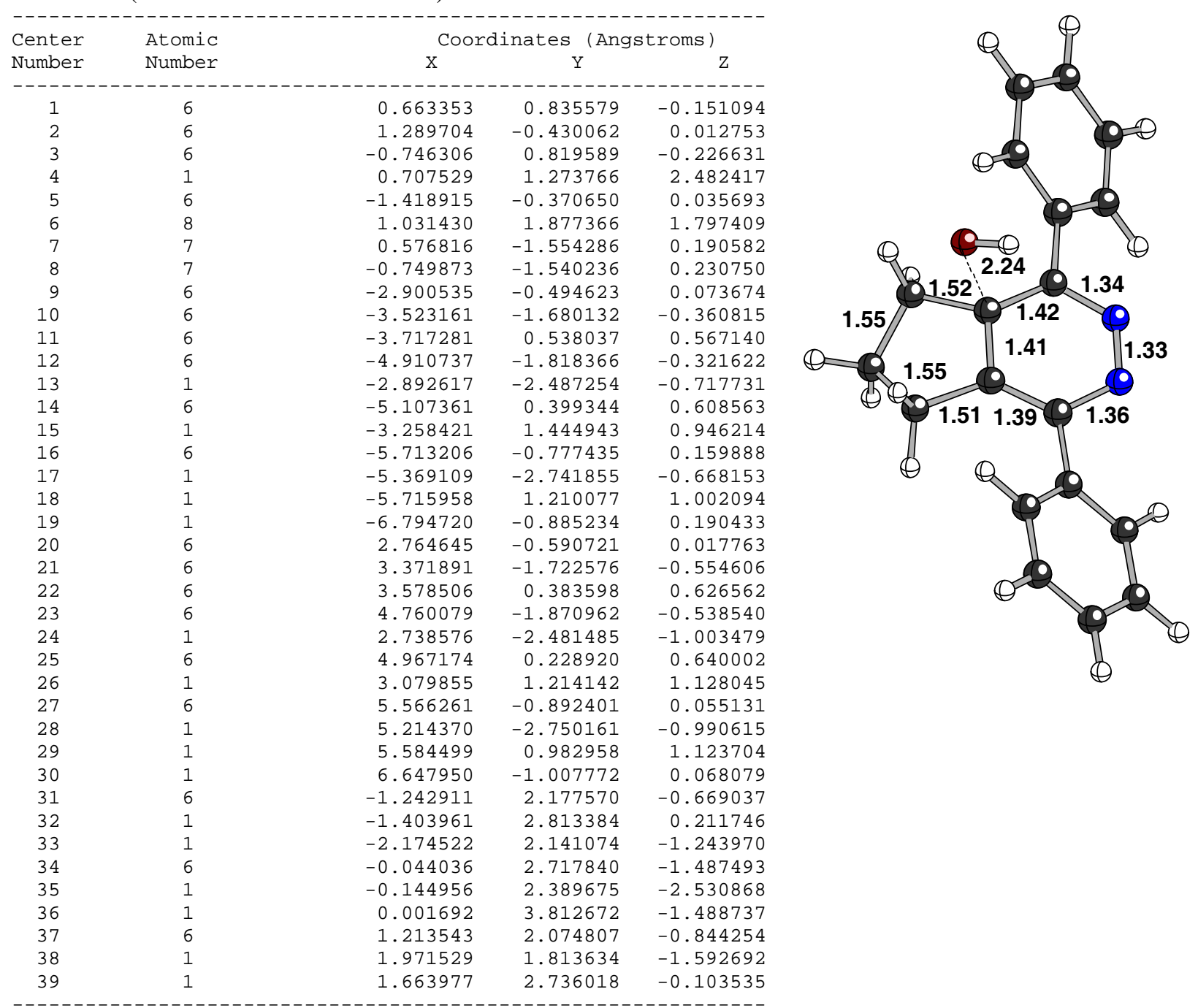


TS12 $\rightarrow$ pyridazines ( $n=2$, pyridazine and scheme figure in SI and footnote 26)

(solvent optimization, methanol, ua0 radii)

$\mathrm{HF}=-919.129248$ hartrees $(-576762.79441248 \mathrm{kcal} / \mathrm{mol})$

Imaginary Frequencies: 1 (-306.8545 1/cm)

Zero-point correction $=0.310504$ (Hartree/Particle)

Temperature 298.150 Kelvin. Pressure 1.00000 Atm.

Sum of electronic and thermal Free Energies = $\mathbf{- 9 1 8 . 8 6 5 5 6 9}$ hartrees $(-\mathbf{5 7 6 5 9 7 . 3 3 3 2 0 3 1 9} \mathbf{k c a l} / \mathrm{mol})$

Coordinates (from last standard orientation):

\begin{tabular}{|c|c|c|c|c|}
\hline \multirow{2}{*}{$\begin{array}{l}\text { Center } \\
\text { Number }\end{array}$} & \multirow{2}{*}{$\begin{array}{l}\text { Atomic } \\
\text { Number }\end{array}$} & \multicolumn{3}{|c|}{ Coordinates (Angstroms) } \\
\hline & & $\mathrm{x}$ & $\mathrm{Y}$ & Z \\
\hline 1 & 6 & 0.664161 & $\odot .911872$ & 0.060106 \\
\hline 2 & 6 & 1.308752 & -0.370305 & 0.187959 \\
\hline 3 & 6 & -0.747524 & 0.831731 & -0.145237 \\
\hline 4 & 1 & 0.404455 & 1.040674 & 2.408585 \\
\hline 5 & 6 & -1.411608 & -0.349047 & $\odot .153846$ \\
\hline 6 & 8 & 0.867476 & 1.711198 & 1.872852 \\
\hline 7 & 7 & $\odot .604057$ & -1.479321 & 0.444540 \\
\hline 8 & 7 & -0.732817 & -1.484010 & 0.494300 \\
\hline 9 & 6 & -2.892697 & -0.492513 & $\odot .085803$ \\
\hline 10 & 6 & -3.476482 & -1.642576 & $-\odot .476719$ \\
\hline 11 & 6 & -3.742806 & 0.505383 & 0.595620 \\
\hline 12 & 6 & -4.865280 & -1.787004 & -0.534143 \\
\hline 13 & 1 & -2.831361 & -2.422294 & -0.875010 \\
\hline 14 & 6 & -5.132481 & 0.360676 & 0.542768 \\
\hline 15 & 1 & -3.316230 & 1.392649 & 1.057249 \\
\hline 16 & 6 & $-5.700 \odot 49$ & -0.785294 & $-\odot .02475 \odot$ \\
\hline 17 & 1 & -5.296721 & -2.682046 & -0.980902 \\
\hline 18 & 1 & -5.771701 & 1.142496 & ๑.951180 \\
\hline 19 & 1 & -6.782539 & -0.897496 & -0.068628 \\
\hline 20 & 6 & 2.779357 & -0.549556 & ๑.070523 \\
\hline 21 & 6 & 3.315329 & -1.734181 & -0.472881 \\
\hline 22 & 6 & 3.676389 & 0.449342 & $\odot .495261$ \\
\hline 23 & 6 & 4.695955 & -1.915269 & -0.583806 \\
\hline 24 & 1 & 2.636838 & -2.511929 & $-\odot .814974$ \\
\hline 25 & 6 & 5.058120 & 0.269330 & 0.382248 \\
\hline 26 & 1 & 3.278763 & 1.352103 & 0.947451 \\
\hline 27 & 6 & 5.576400 & -0.912874 & -0.158283 \\
\hline 28 & 1 & 5.085581 & -2.838178 & -1.012150 \\
\hline 29 & 1 & 5.732259 & 1.053194 & 0.725969 \\
\hline 30 & 1 & 6.652964 & -1.052018 & -0.247271 \\
\hline 31 & 6 & -1.256979 & 2.119908 & -0.750615 \\
\hline 32 & 1 & -1.544620 & 2.833913 & 0.034652 \\
\hline 33 & 1 & -2.129379 & 1.981151 & -1.397262 \\
\hline 34 & 6 & -0.014309 & 2.640481 & -1.511863 \\
\hline 35 & 1 & -0.006123 & 2.212926 & -2.522274 \\
\hline 36 & 1 & -0.009003 & 3.730603 & -1.616645 \\
\hline 37 & 6 & 1.206643 & 2.117863 & -0.710083 \\
\hline 38 & 1 & 2.036388 & 1.838843 & -1.367583 \\
\hline 39 & 1 & 1.576591 & 2.871344 & -0.011609 \\
\hline
\end{tabular}

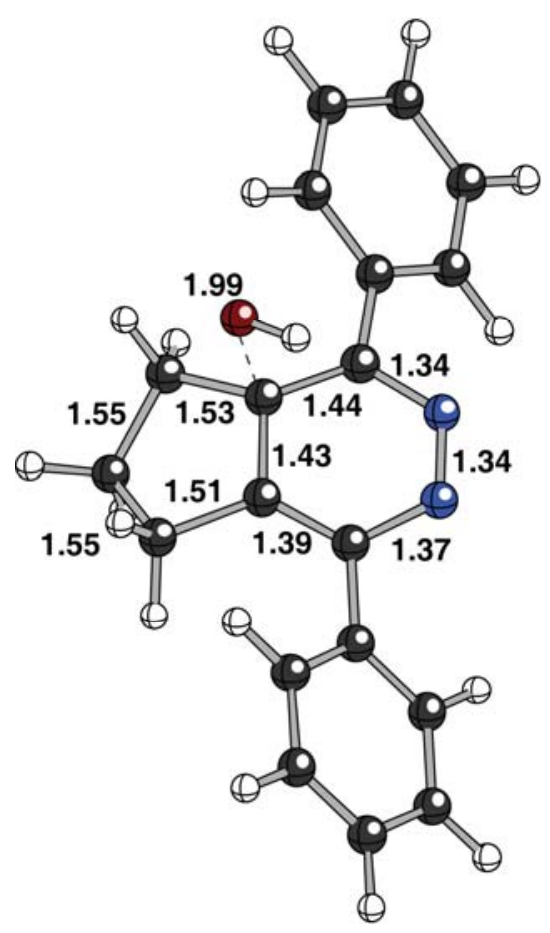




\section{Pyridazine ( $\mathrm{n}=\mathbf{1}$, pyridazine scheme in SI and footnote 26)}

$\mathrm{HF}=-803.8381319$ hartrees $(-504416.466148569 \mathrm{kcal} / \mathrm{mol})$

Imaginary Frequencies: none found

Zero-point correction $=0.271534$ (Hartree/Particle)

Temperature 298.150 Kelvin. Pressure 1.00000 Atm.

Sum of electronic and thermal Free Energies = -803.613434 hartrees $(-504275.46596934 \mathrm{kcal} / \mathrm{mol})$

Coordinates (from last standard orientation):

\begin{tabular}{|c|c|c|c|c|}
\hline Center & Atomic & $\mathrm{CoO}$ & inates (An & troms ) \\
\hline Number & Number & $x$ & $\mathrm{Y}$ & z \\
\hline 1 & 6 & -0.790274 & 2.589419 & $\odot .0 \odot \odot 277$ \\
\hline 2 & 1 & -1.248407 & 3.030951 & $\odot .891418$ \\
\hline 3 & 1 & -1.249396 & 3.031894 & $-\odot .889825$ \\
\hline 4 & 6 & 0.789832 & 2.589482 & $-\odot . \odot \odot \odot 221$ \\
\hline 5 & 1 & 1.248943 & 3.031605 & ๑. 890112 \\
\hline 6 & 1 & 1.247965 & 3.031481 & $-\odot .891136$ \\
\hline 7 & 6 & -0.691820 & 1.070238 & $-\odot . \odot \odot \odot 189$ \\
\hline 8 & 6 & -1.395648 & -0.137900 & $-\odot .000159$ \\
\hline 9 & 6 & 0.691634 & 1.070331 & $-\odot . \odot \odot \odot 361$ \\
\hline 10 & 6 & 1.395657 & -0.137747 & $-\odot .00 \odot 458$ \\
\hline 11 & 7 & -0.660240 & -1.278692 & $-\odot .00 \odot 499$ \\
\hline 12 & 7 & $0.66 \odot 297$ & -1.278583 & $-\odot . \odot \odot \odot 715$ \\
\hline 13 & 6 & 2.868129 & -0.288541 & -0.000116 \\
\hline 14 & 6 & 3.453740 & -1.568256 & ๑. 005376 \\
\hline 15 & 6 & 3.712415 & 0.836248 & $-\odot .005295$ \\
\hline 16 & 6 & 4.839641 & -1.713130 & $\odot .005854$ \\
\hline 17 & 1 & 2.804399 & -2.435877 & ๑.009219 \\
\hline 18 & 6 & 5.100245 & 0.687866 & $-\odot .0 \odot 4856$ \\
\hline 19 & 1 & 3.290499 & 1.835181 & $-\odot .0 \odot 9914$ \\
\hline 20 & 6 & 5.670333 & -0.587414 & $\odot .0 \odot \odot 767$ \\
\hline 21 & 1 & 5.274104 & -2.708757 & ๑. 010257 \\
\hline 22 & 1 & 5.734900 & 1.569439 & $-\odot .0 \odot 8997$ \\
\hline 23 & 1 & 6.750304 & -0.703382 & ๑. .0०1155 \\
\hline 24 & 6 & -2.868061 & -0.288706 & ๑. $00 \odot \odot 1 \odot$ \\
\hline 25 & 6 & -3.453716 & -1.568409 & $-\odot . \odot \odot 5183$ \\
\hline 26 & 6 & -3.712232 & 0.836132 & $\odot .005430$ \\
\hline 27 & 6 & -4.839620 & -1.713151 & $-\odot .005264$ \\
\hline 28 & 1 & -2.804430 & -2.436080 & $-\odot .009248$ \\
\hline 29 & 6 & -5.100068 & 0.687901 & $\odot .005336$ \\
\hline 30 & 1 & -3.290008 & 1.834955 & $\odot .0 \odot 9855$ \\
\hline 31 & 6 & -5.670224 & -0.587359 & -0.000016 \\
\hline 32 & 1 & -5.274190 & -2.708735 & $-\odot .009467$ \\
\hline 33 & 1 & -5.734660 & 1.569515 & ๑.๑๑9569 \\
\hline 34 & 1 & -6.750198 & $-\odot .703278$ & $-\odot . \odot \odot \odot \odot 94$ \\
\hline
\end{tabular}

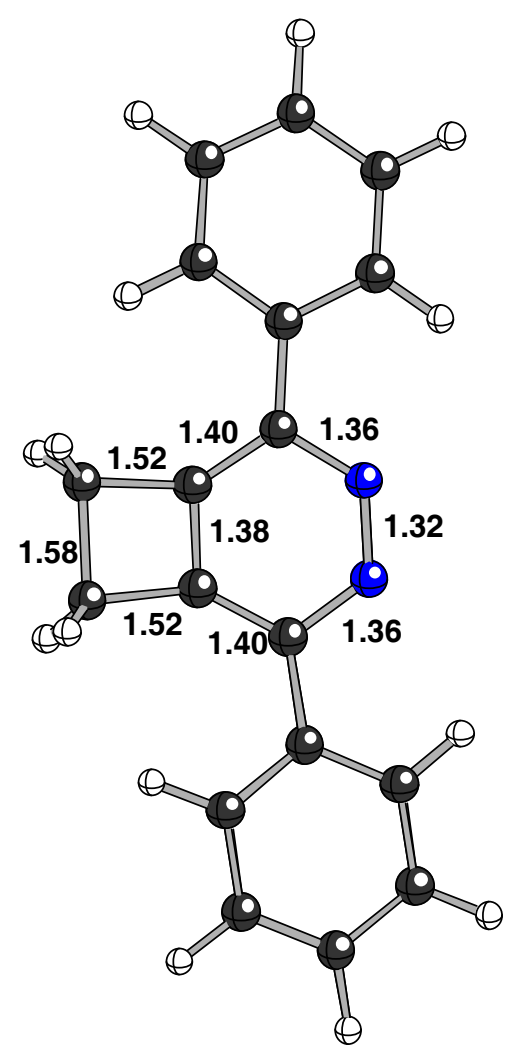




\section{Pyridazine ( $n=1$, pyridazine scheme in SI and footnote 26$)$}

(solvent optimization, methanol, uaks radii)

$\mathrm{HF}=-803.8548721$ hartrees $(-504426.970791471 \mathrm{kcal} / \mathrm{mol})$

Imaginary Frequencies: none found

Zero-point correction $=0.270376$ (Hartree/Particle)

Temperature 298.150 Kelvin. Pressure 1.00000 Atm.

Sum of electronic and thermal Free Energies $=\mathbf{- 8 0 3 . 6 2 9 0 7 5}$ hartrees $(-504285.28085325 \mathrm{kcal} / \mathbf{m o l})$

Coordinates (from last standard orientation):

\begin{tabular}{|c|c|c|c|c|}
\hline \multirow{2}{*}{$\begin{array}{l}\text { Center } \\
\text { Number }\end{array}$} & \multirow{2}{*}{$\begin{array}{l}\text { Atomic } \\
\text { Number }\end{array}$} & \multicolumn{3}{|c|}{ Coordinates (Angstroms) } \\
\hline & & $\mathrm{x}$ & $\mathrm{Y}$ & Z \\
\hline 1 & 6 & -0.791228 & 2.539292 & $\odot .326846$ \\
\hline 2 & 1 & -1.256760 & 2.820687 & 1.279889 \\
\hline 3 & 1 & -1.246131 & 3.122980 & -0.483016 \\
\hline 4 & 6 & 0.789560 & 2.539727 & $\odot .327913$ \\
\hline 5 & 1 & 1.253769 & 2.821758 & 1.281410 \\
\hline 6 & 1 & 1.245123 & 3.123379 & $-\odot .481634$ \\
\hline 7 & 6 & -0.691693 & 1.042279 & $\odot .078121$ \\
\hline 8 & 6 & -1.395594 & -0.158300 & $-\odot .069320$ \\
\hline 9 & 6 & 0.691227 & 1.042559 & 0.079522 \\
\hline 10 & 6 & 1.395700 & -0.158028 & $-\odot .065375$ \\
\hline 11 & 7 & -0.664257 & -1.289836 & -0.223056 \\
\hline 12 & 7 & 0.664821 & -1.289717 & -0.219498 \\
\hline 13 & 6 & 2.870105 & -0.295080 & $-\odot .050076$ \\
\hline 14 & 6 & 3.477191 & -1.542532 & 0.187652 \\
\hline 15 & 6 & 3.694344 & 0.824315 & $-\odot .268143$ \\
\hline 16 & 6 & 4.866695 & -1.664305 & ๑. 205938 \\
\hline 17 & 1 & 2.851075 & -2.411349 & 0.362416 \\
\hline 18 & 6 & 5.085296 & 0.699541 & $-\odot .251830$ \\
\hline 19 & 1 & 3.254042 & 1.795717 & $-\odot .46800 \odot$ \\
\hline 20 & 6 & 5.677355 & -0.544469 & -0.013922 \\
\hline 21 & 1 & 5.318630 & -2.634999 & ๑.396049 \\
\hline 22 & 1 & 5.705106 & 1.575285 & -0.428453 \\
\hline 23 & 1 & 6.760321 & -0.641148 & ๑. 001050 \\
\hline 24 & 6 & -2.869916 & -0.295556 & -0.053938 \\
\hline 25 & 6 & -3.476457 & -1.541338 & $\odot .193650$ \\
\hline 26 & 6 & -3.694509 & 0.822310 & $-\odot .278429$ \\
\hline 27 & 6 & -4.865946 & -1.662983 & 0.215458 \\
\hline 28 & 1 & -2.849963 & -2.408769 & $\odot .373904$ \\
\hline 29 & 6 & -5.085417 & 0.697634 & -0.258575 \\
\hline 30 & 1 & -3.254438 & 1.792287 & -0.485878 \\
\hline 31 & 6 & -5.676989 & -0.544680 & -0.010656 \\
\hline 32 & 1 & -5.317536 & -2.632221 & 0.413680 \\
\hline 33 & 1 & -5.705612 & 1.572108 & -0.440110 \\
\hline 34 & 1 & -6.759926 & -0.641152 & $\odot .007543$ \\
\hline
\end{tabular}

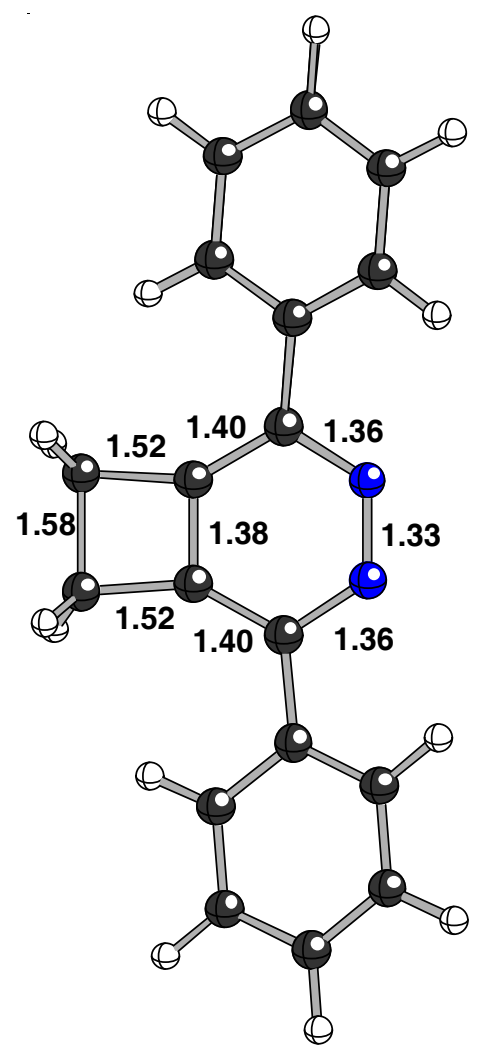




\section{$\mathrm{OH}^{-}$(pyridazine scheme in SI and footnote 26)}

$\mathrm{HF}=-75.8034349$ hartrees $(-47567.413434099 \mathrm{kcal} / \mathrm{mol})$

Imaginary Frequencies: none found

Zero-point correction $=0.008554$ (Hartree/Particle)

Temperature 298.150 Kelvin. Pressure 1.00000 Atm.

Sum of electronic and thermal Free Energies $=-75.811143$ hartrees $(-47572.25034393 \mathrm{kcal} / \mathrm{mol})$

Coordinates (from last standard orientation):

\begin{tabular}{|c|c|c|c|c|}
\hline Center & Atomic & Coo & nates (An & roms ) \\
\hline Number & Number & X & Y & Z \\
\hline $\begin{array}{l}1 \\
2\end{array}$ & $\begin{array}{l}8 \\
1\end{array}$ & 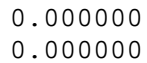 & 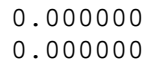 & $\begin{array}{r}0.107768 \\
-0.862143\end{array}$ \\
\hline
\end{tabular}


$\mathrm{OH}^{-}$(pyridazine scheme in SI and footnote 26)

(solvent optimization, methanol, uaks radii)

$\mathrm{HF}=-76.0389539 \mathrm{hartrees}(-47715.203961789 \mathrm{kcal} / \mathrm{mol})$

Imaginary Frequencies: none found

Zero-point correction $=0.013515$ (Hartree/Particle)

Temperature 298.150 Kelvin. Pressure 1.00000 Atm.

Sum of electronic and thermal Free Energies = -76.042483 hartrees $(-47717.41850733 \mathrm{kcal} / \mathrm{mol})$

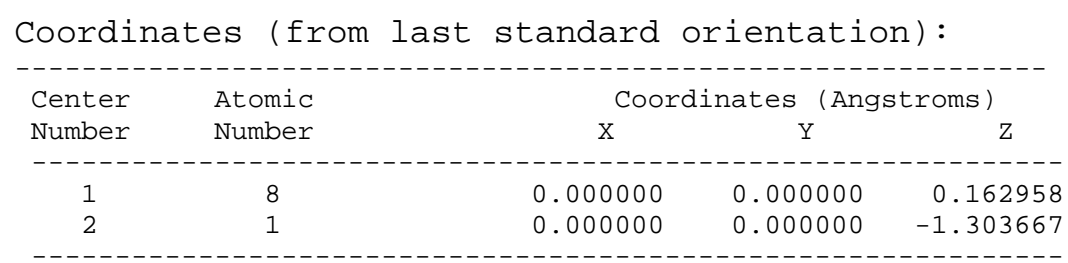

1.47

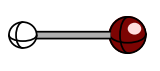


4 ( $n=2$, pyridazine scheme in SI and footnote 26$)$

See cyclopentanone reactant in Table 2. 
TS4 $\rightarrow 6$ ( $n=2$, pyridazine scheme in SI and footnote 26$)$

See cyclopentanone Electrocyclic TS in Table 2. 


\section{$6(\mathrm{n}=2$, pyridazine figure and scheme in $\mathrm{SI})$}

$\mathrm{HF}=-919.0190476$ hartrees $(-576693.642559476 \mathrm{kcal} / \mathrm{mol})$

Imaginary Frequencies: none found

Zero-point correction $=0.311949$ (Hartree/Particle)

Temperature 298.150 Kelvin. Pressure 1.00000 Atm.

Sum of electronic and thermal Free Energies $=\mathbf{- 9 1 8 . 7 5 4 7 8 6}$ hartrees $(-576527.81576286 \mathrm{kcal} / \mathrm{mol})$

Coordinates (from last standard orientation):

\begin{tabular}{|c|c|c|c|c|}
\hline \multirow{2}{*}{$\begin{array}{l}\text { Center } \\
\text { Number }\end{array}$} & \multirow{2}{*}{$\begin{array}{l}\text { Atomic } \\
\text { Number }\end{array}$} & \multicolumn{3}{|c|}{ Coordinates (Angstroms) } \\
\hline & & $\mathrm{X}$ & $\mathrm{Y}$ & Z \\
\hline-- & & ------- & 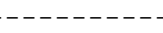 &.----- \\
\hline 1 & 6 & $\odot .613144$ & 0.407309 & 1.139950 \\
\hline 2 & 6 & 1.209308 & -0.232764 & -0.095865 \\
\hline 3 & 6 & -1.347651 & 1.711457 & -0.582736 \\
\hline 4 & 1 & -2.102515 & 2.315368 & -0.082374 \\
\hline 5 & 6 & -1.592294 & 0.372076 & -0.731664 \\
\hline 6 & 8 & 0.115436 & -0.271570 & 2.034116 \\
\hline 7 & 7 & 0.523675 & -0.650216 & -1.157452 \\
\hline 8 & 7 & -0.769965 & -0.550116 & -1.372670 \\
\hline 9 & 6 & -2.922265 & $-\odot .187807$ & -0.331064 \\
\hline 10 & 6 & -3.001157 & -1.513080 & ๑.131308 \\
\hline 11 & 6 & -4.113244 & $\odot .555725$ & -0.404674 \\
\hline 12 & 6 & -4.218964 & -2.063122 & 0.534700 \\
\hline 13 & 1 & -2.087527 & -2.096468 & ๑.170552 \\
\hline 14 & 6 & -5.334103 & ๑. 008118 & $-\odot .002901$ \\
\hline 15 & 1 & -4.082538 & 1.567133 & -0.799711 \\
\hline 16 & 6 & -5.393704 & -1.305714 & ๑. 472694 \\
\hline 17 & 1 & -4.251205 & -3.086968 & 0.900238 \\
\hline 18 & 1 & -6.241925 & ๑. 603515 & $-\odot .074504$ \\
\hline 19 & 1 & -6.343449 & -1.735836 & 0.782169 \\
\hline 20 & 6 & 2.643712 & -0.509263 & -0.076096 \\
\hline 21 & 6 & 3.325223 & -0.951812 & -1.242357 \\
\hline 22 & 6 & 3.431510 & -0.340839 & 1.091359 \\
\hline 23 & 6 & 4.691167 & -1.209152 & -1.231696 \\
\hline 24 & 1 & 2.741159 & -1.089914 & -2.145601 \\
\hline 25 & 6 & 4.805398 & $-\odot .590546$ & 1.090765 \\
\hline 26 & 1 & 2.956404 & $-\odot .043881$ & 2.021836 \\
\hline 27 & 6 & 5.455671 & -1.027257 & $-\odot .067441$ \\
\hline 28 & 1 & 5.173401 & -1.550209 & -2.146343 \\
\hline 29 & 1 & 5.369043 & $-\odot .453381$ & 2. 011826 \\
\hline 30 & 1 & 6.524995 & -1.222855 & -0.066550 \\
\hline 31 & 6 & -0.109809 & 2.417990 & -1.059200 \\
\hline 32 & 1 & -0.396402 & 3.348501 & -1.571533 \\
\hline 33 & 1 & ๑. 384567 & 1.792369 & -1.807188 \\
\hline 34 & 6 & 0.907618 & 2.775212 & 0.071130 \\
\hline 35 & 1 & 1.928378 & 2.691477 & -0.320258 \\
\hline 36 & 1 & 0.778904 & 3.825541 & 0.366480 \\
\hline 37 & 6 & 0.762562 & 1.919313 & 1.340732 \\
\hline 38 & 1 & 1.660690 & 2.052575 & 1.963109 \\
\hline 39 & 1 & -0.084175 & 2.242844 & 1.954107 \\
\hline
\end{tabular}

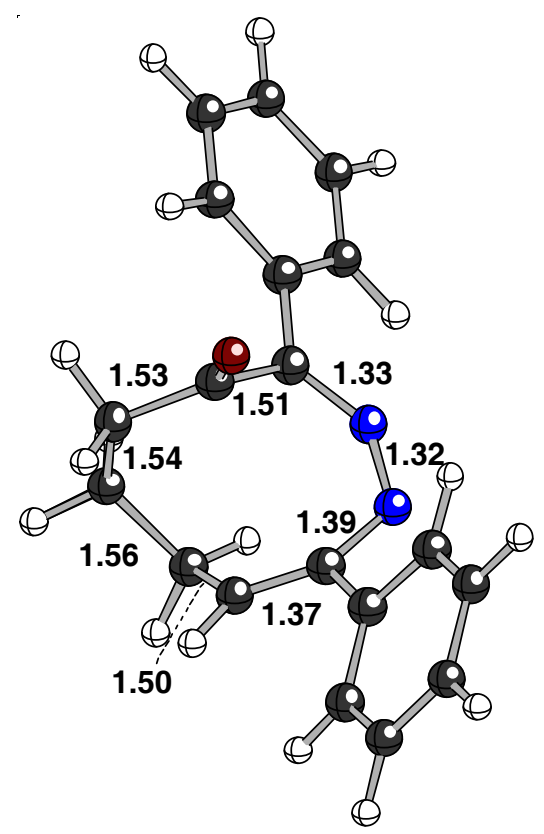




\section{$6(n=2$, pyridazine figure and scheme in SI)}

\section{(solvent optimization, methanol, uaks radii)}

$\mathrm{HF}=-919.104032$ hartrees $(-576746.97112032 \mathrm{kcal} / \mathrm{mol})$

Imaginary Frequencies: none found

Zero-point correction $=0.311605$ (Hartree/Particle)

Temperature 298.150 Kelvin. Pressure 1.00000 Atm.

Sum of electronic and thermal Free Energies $=\mathbf{- 9 1 8 . 8 3 9 8 6 1}$ hartrees $(-576581.20117611 \mathrm{kcal} / \mathrm{mol})$

Coordinates (from last standard orientation):

\begin{tabular}{|c|c|c|c|c|}
\hline \multirow{2}{*}{$\begin{array}{l}\text { Center } \\
\text { Number }\end{array}$} & \multirow{2}{*}{$\begin{array}{l}\text { Atomic } \\
\text { Number }\end{array}$} & \multicolumn{3}{|c|}{ Coordinates (Angstroms) } \\
\hline & & $\mathrm{x}$ & $\mathrm{Y}$ & Z \\
\hline 1 & 6 & $\odot .599674$ & $\odot .370569$ & 1.112449 \\
\hline 2 & 6 & 1.207485 & -0.230123 & -0.138637 \\
\hline 3 & 6 & -1.344938 & 1.719132 & $-\odot .525659$ \\
\hline 4 & 1 & -2.088254 & 2.294312 & 0.023947 \\
\hline 5 & 6 & -1.598844 & $\odot .394696$ & -0.736998 \\
\hline 6 & 8 & 0.106790 & -0.364894 & 1.971911 \\
\hline 7 & 7 & 0.533268 & -0.610360 & -1.213038 \\
\hline 8 & 7 & -0.772274 & -0.495878 & -1.446555 \\
\hline 9 & 6 & -2.920423 & -0.183937 & -0.336835 \\
\hline 10 & 6 & -3.000293 & -1.523751 & ๑. 085017 \\
\hline 11 & 6 & -4.110108 & 0.568150 & -0.371127 \\
\hline 12 & 6 & -4.216208 & -2.084322 & 0.484833 \\
\hline 13 & 1 & -2.092451 & -2.119377 & 0.102855 \\
\hline 14 & 6 & -5.327932 & 0.010672 & $\odot .027693$ \\
\hline 15 & 1 & -4.085069 & 1.593789 & -0.730137 \\
\hline 16 & 6 & -5.387279 & -1.318779 & 0.460550 \\
\hline 17 & 1 & -4.249484 & -3.119974 & ๑. 816873 \\
\hline 18 & 1 & -6.234019 & 0.611658 & -0.012962 \\
\hline 19 & 1 & -6.335598 & -1.754969 & 0.766297 \\
\hline 20 & 6 & 2.652659 & -0.502909 & -0.101912 \\
\hline 21 & 6 & 3.368011 & $-\odot .860740$ & -1.273008 \\
\hline 22 & 6 & 3.399869 & -0.408721 & 1. 097281 \\
\hline 23 & 6 & 4.735745 & -1.121056 & -1.238978 \\
\hline 24 & 1 & 2.825335 & -0.929436 & -2.210112 \\
\hline 25 & 6 & 4.774470 & -0.660126 & 1.124391 \\
\hline 26 & 1 & 2.902126 & -0.161224 & 2.030848 \\
\hline 27 & 6 & 5.458101 & -1.020817 & -0.040560 \\
\hline 28 & 1 & 5.248090 & -1.395343 & -2.159467 \\
\hline 29 & 1 & 5.309996 & -0.580611 & 2.068482 \\
\hline 30 & 1 & 6.527301 & -1.216552 & -0.019348 \\
\hline 31 & 6 & -0.122578 & 2.461834 & -0.986262 \\
\hline 32 & 1 & -0.431502 & 3.411595 & -1.444701 \\
\hline 33 & 1 & 0.374165 & 1.884364 & -1.771631 \\
\hline 34 & 6 & $\odot .894558$ & 2.776834 & $\odot .156007$ \\
\hline 35 & 1 & 1.917636 & 2.725197 & -0.242781 \\
\hline 36 & 1 & $\odot .752262$ & 3.810773 & 0.503948 \\
\hline 37 & 6 & 0.755223 & 1.861334 & 1.383787 \\
\hline 38 & 1 & 1.658709 & 1.958124 & 2.006903 \\
\hline 39 & 1 & -0.083678 & 2.162853 & 2.020662 \\
\hline
\end{tabular}

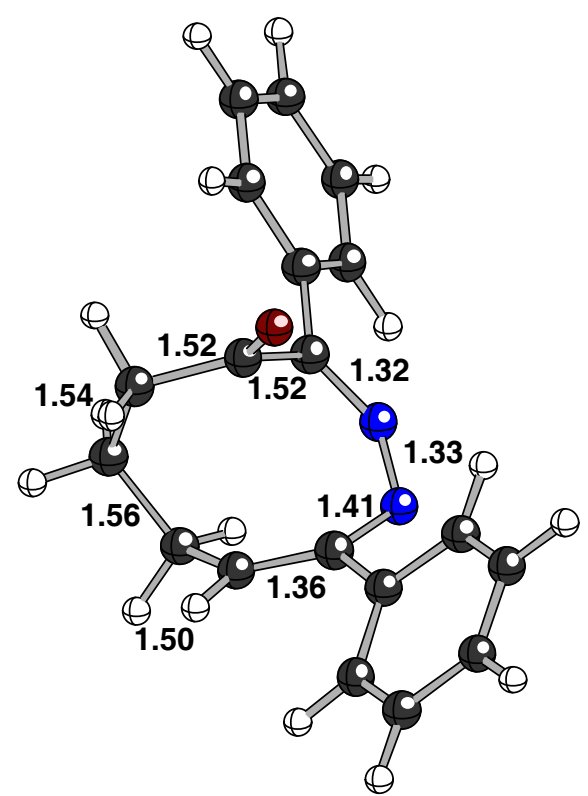




\section{Pyridazine ( $n=2$, pyridazine scheme in SI and footnote 26)}

$\mathrm{HF}=-843.1963106$ hartrees $(-529114.116864606 \mathrm{kcal} / \mathrm{mol})$

Imaginary Frequencies: none found

Zero-point correction $=0.301639$ (Hartree/Particle)

Temperature 298.150 Kelvin. Pressure 1.00000 Atm.

Sum of electronic and thermal Free Energies = -842.939321 hartrees $(-528952.85332071 \mathrm{kcal} / \mathrm{mol})$

Coordinates (from last standard orientation):

\begin{tabular}{|c|c|c|c|c|}
\hline \multirow{2}{*}{$\begin{array}{l}\text { Center } \\
\text { Number }\end{array}$} & \multirow{2}{*}{$\begin{array}{l}\text { Atomic } \\
\text { Number }\end{array}$} & \multicolumn{3}{|c|}{ Coordinates (Angstroms) } \\
\hline & & $\mathrm{x}$ & $\mathrm{Y}$ & Z \\
\hline 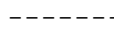 & & 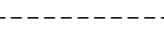 & 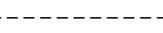 &.----- \\
\hline 1 & 6 & -0.696962 & ๑. 946336 & 0.063440 \\
\hline 2 & 6 & -1.365823 & $-\odot .281594$ & -0.073975 \\
\hline 3 & 6 & ๑. 696963 & 0.946355 & 0.063413 \\
\hline 4 & 6 & 1.365815 & -0.281586 & -0.073999 \\
\hline 5 & 7 & -0.659387 & -1.432347 & -0.142799 \\
\hline 6 & 7 & ๑. 659396 & -1.432337 & -0.142799 \\
\hline 7 & 6 & 2.842600 & -0.441708 & -0.097143 \\
\hline 8 & 6 & 3.426698 & -1.587791 & 0.471804 \\
\hline 9 & 6 & 3.681874 & 0.511572 & $-\odot .697918$ \\
\hline 10 & 6 & 4.809575 & -1.763613 & ๑. 454696 \\
\hline 11 & 1 & 2.780582 & -2.334919 & 0.919118 \\
\hline 12 & 6 & 5.066487 & 0.330362 & -0.721255 \\
\hline 13 & 1 & 3.255592 & 1.385837 & -1.177943 \\
\hline 14 & 6 & 5.636128 & $-\odot .804759$ & -0.140105 \\
\hline 15 & 1 & 5.243520 & -2.651732 & ๑. 905198 \\
\hline 16 & 1 & 5.697721 & 1.073345 & -1.200357 \\
\hline 17 & 1 & 6.713276 & -0.943911 & -0.154602 \\
\hline 18 & 6 & -2.842600 & -0.441714 & $-\odot .097129$ \\
\hline 19 & 6 & -3.426723 & -1.587620 & 0.472120 \\
\hline 20 & 6 & -3.681830 & 0.511416 & -0.698218 \\
\hline 21 & 6 & -4.809610 & -1.763439 & 0.455000 \\
\hline 22 & 1 & -2.780636 & -2.334623 & ○. 919679 \\
\hline 23 & 6 & -5.066437 & ๑. 330206 & -0.721558 \\
\hline 24 & 1 & -3.255517 & 1.385543 & -1.178452 \\
\hline 25 & 6 & -5.636118 & -0.804747 & -0.140110 \\
\hline 26 & 1 & -5.243591 & -2.651424 & $\odot .905731$ \\
\hline 27 & 1 & -5.697656 & 1.073055 & -1.200897 \\
\hline 28 & 1 & -6.713267 & -0.943884 & $-\odot .154615$ \\
\hline 29 & 6 & 1.225814 & 2.346652 & ๑. 301886 \\
\hline 30 & 1 & 1.522486 & 2.810031 & -0.649524 \\
\hline 31 & 1 & 2.105090 & 2.365438 & ๑. 952257 \\
\hline 32 & 6 & -0.000020 & 3.078401 & ๑. 899930 \\
\hline 33 & 1 & $-0.0 \odot \odot \odot 28$ & 2.957697 & 1.989317 \\
\hline 34 & 1 & $-0.0 \odot \odot \odot 25$ & 4.150683 & 0.685304 \\
\hline 35 & 6 & -1.225840 & 2.346644 & 0.301860 \\
\hline 36 & 1 & -2.105158 & 2.365469 & ○. 952154 \\
\hline 37 & 1 & -1.522401 & 2.809956 & -0.649609 \\
\hline
\end{tabular}

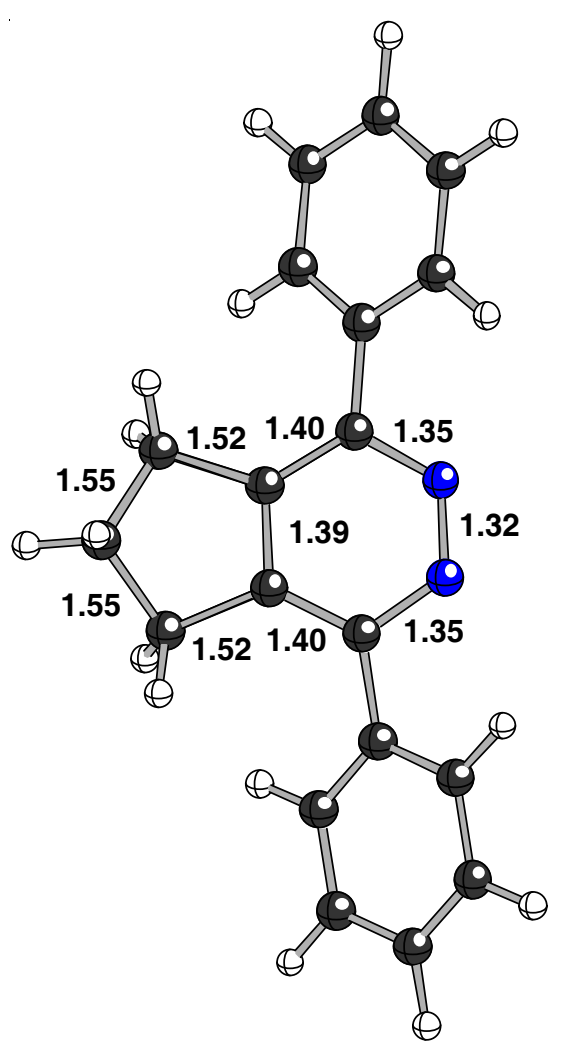




\section{Pyridazine ( $n=2$, pyridazine scheme in SI and footnote 26$)$}

\section{(solvent optimization, methanol, uaks radii)}

$\mathrm{HF}=-843.2146474$ hartrees $(-529125.623389974 \mathrm{kcal} / \mathrm{mol})$

Imaginary Frequencies: none found

Zero-point correction $=0.300117$ (Hartree/Particle)

Temperature 298.150 Kelvin. Pressure 1.00000 Atm.

Sum of electronic and thermal Free Energies $=\mathbf{- 8 4 2 . 9 5 9 3 7 8 ~ h a r t r e e s ~}(-528965.43928878 \mathrm{kcal} / \mathrm{mol})$

Coordinates (from last standard orientation):

\begin{tabular}{|c|c|c|c|c|}
\hline Center & Atomic & $\mathrm{CoO}$ & inates (Ang & troms ) \\
\hline Number & Number & $x$ & $Y$ & Z \\
\hline 1 & 6 & -0.696509 & 0.918685 & 0.076027 \\
\hline 2 & 6 & -1.365744 & -0.304797 & -0.102843 \\
\hline 3 & 6 & 0.696152 & 0.918928 & 0.076985 \\
\hline 4 & 6 & 1.366032 & -0.304494 & -0.099557 \\
\hline 5 & 7 & -0.663355 & -1.449978 & -0.237502 \\
\hline 6 & 7 & 0.664207 & -1.449934 & $-0.23432 \varepsilon$ \\
\hline 7 & 6 & 2.845741 & -0.447411 & -0.108124 \\
\hline 8 & 6 & 3.452011 & -1.525533 & $\odot .561026$ \\
\hline 9 & 6 & 3.662549 & $\odot .469691$ & -0.791340 \\
\hline 10 & 6 & 4.839630 & -1.677385 & 0.553315 \\
\hline 11 & 1 & 2.828209 & -2.238740 & 1.092058 \\
\hline 12 & 6 & 5.050933 & 0.312098 & -0.805214 \\
\hline 13 & 1 & 3.215814 & 1.295612 & $-1.33720 s$ \\
\hline 14 & 6 & 5.644357 & -0.759066 & $-0.13020 \mathrm{~s}$ \\
\hline 15 & 1 & 5.293037 & -2.511769 & $1.08325 \mathrm{c}$ \\
\hline 16 & 1 & 5.667316 & 1. 024861 & -1.347837 \\
\hline 17 & 1 & 6.725193 & -0.878126 & -0.137326 \\
\hline 18 & 6 & -2.845388 & $-\odot .448329$ & -0.111351 \\
\hline 19 & 6 & -3.450482 & -1.526675 & 0.558581 \\
\hline 20 & 6 & -3.663424 & ๑. 469154 & -0.792567 \\
\hline 21 & 6 & -4.838152 & -1.678212 & 0.553856 \\
\hline 22 & 1 & -2.825729 & -2.239959 & 1.088374 \\
\hline 23 & 6 & -5.051866 & $\odot .311827$ & -0.803525 \\
\hline 24 & 1 & -3.217705 & 1.295417 & -1.338745 \\
\hline 25 & 6 & -5.644106 & -0.759375 & -0.127513 \\
\hline 26 & 1 & -5.290623 & -2.512565 & 1.084664 \\
\hline 27 & 1 & -5.669318 & 1.025030 & -1.344376 \\
\hline 28 & 1 & -6.725005 & -0.877981 & $-0.13201 \varepsilon$ \\
\hline 29 & 6 & 1.226825 & 2.309734 & 0.349172 \\
\hline 30 & 1 & 1.529390 & 2.785991 & -0.594966 \\
\hline 31 & 1 & 2.103385 & 2.310631 & 1.005456 \\
\hline 32 & 6 & $-\odot .00 \odot 967$ & 3.032126 & 0.957574 \\
\hline 33 & 1 & $-\odot .001551$ & $2.8950 \odot 2$ & 2.049162 \\
\hline 34 & 1 & $-\odot . \odot \odot \odot 968$ & 4.109604 & 0.750160 \\
\hline 35 & 6 & -1.227969 & 2.309250 & 0.348122 \\
\hline 36 & 1 & -2.104960 & 2.309593 & 1.003827 \\
\hline 37 & 1 & -1.530177 & 2.785496 & -0.596131 \\
\hline
\end{tabular}

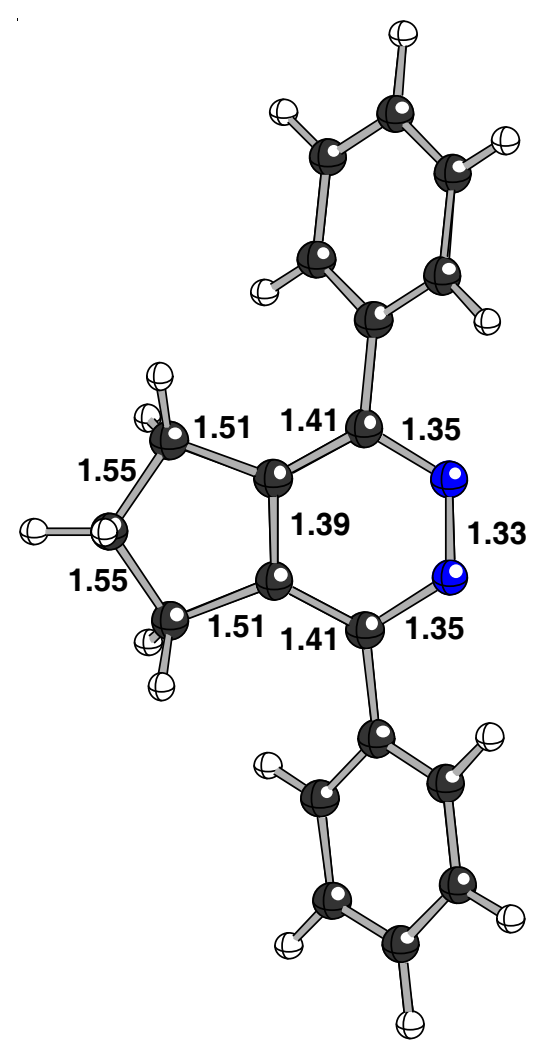




\section{bis-p-CN substituted reactant $(\mathrm{n}=\mathbf{1}$, footnote 25$)$}

$\mathrm{HF}=-1064.2063579$ hartrees $(-667800.131645829 \mathrm{kcal} / \mathrm{mol})$

Imaginary Frequencies: none found

Zero-point correction $=0.281320$ (Hartree/Particle)

Temperature 298.150 Kelvin. Pressure 1.00000 Atm.

Sum of electronic and thermal Free Energies = -1063.975467 hartrees (-667655.24529717 $\mathrm{kcal} / \mathrm{mol})$

\begin{tabular}{|c|c|c|c|c|}
\hline \multirow{2}{*}{$\begin{array}{l}\text { Center } \\
\text { Number }\end{array}$} & \multirow{2}{*}{$\begin{array}{l}\text { Atomic } \\
\text { Number }\end{array}$} & \multicolumn{3}{|c|}{ Coordinates (Angstroms) } \\
\hline & & $\mathrm{X}$ & $\mathrm{Y}$ & Z \\
\hline 1 & 6 & -0.997643 & 2.355706 & 0.780222 \\
\hline 2 & 1 & -1.940596 & 2.278057 & 1.333910 \\
\hline 3 & 1 & -0.903195 & 3.365747 & $\odot .365174$ \\
\hline 4 & 6 & 0.300633 & 1.893312 & 1.480540 \\
\hline 5 & 1 & 0.120212 & 1.104686 & 2.218198 \\
\hline 6 & 1 & 0.937416 & 2.656762 & 1.940855 \\
\hline 7 & 6 & -0.705066 & 1.324346 & $-\odot .384941$ \\
\hline 8 & 6 & -1.288397 & $-\odot .061681$ & -0.077224 \\
\hline 9 & 6 & 0.808693 & 1.302943 & 0.126351 \\
\hline 10 & 1 & 1.363593 & 2.057909 & -0.439724 \\
\hline 11 & 6 & 1.458777 & -0.027578 & 0.094510 \\
\hline 12 & 8 & -0.975622 & 1.595012 & -1.650747 \\
\hline 13 & 7 & -0.600416 & -1.160632 & $\odot .103737$ \\
\hline 14 & 7 & 0.785842 & -1.145994 & 0.137363 \\
\hline 15 & 6 & 2.933311 & $-\odot .172656$ & ๑. 019836 \\
\hline 16 & 6 & 3.524742 & -1.436561 & $-\odot .199826$ \\
\hline 17 & 6 & 3.788007 & 0.935639 & ๑.181331 \\
\hline 18 & 6 & 4.902150 & -1.586711 & -0.252733 \\
\hline 19 & 1 & 2.867164 & -2.288878 & -0.325664 \\
\hline 20 & 6 & 5.171936 & $\odot .797270$ & ๑.127973 \\
\hline 21 & 1 & 3.365201 & 1.918144 & 0.358964 \\
\hline 22 & 6 & 5.745455 & -0.467345 & -0.089434 \\
\hline 23 & 1 & 5.340611 & -2.564895 & -0.425086 \\
\hline 24 & 1 & 5.813993 & 1.663134 & $\odot .256176$ \\
\hline 25 & 6 & -2.761774 & $-\odot .242947$ & $-\odot .113331$ \\
\hline 26 & 6 & -3.344195 & -1.320126 & 0.587612 \\
\hline 27 & 6 & -3.614888 & 0.661411 & -0.779557 \\
\hline 28 & 6 & -4.722540 & -1.489799 & 0.631206 \\
\hline 29 & 1 & -2.687598 & -2.019930 & 1.092055 \\
\hline 30 & 6 & -4.995399 & 0.503372 & $-\odot .732181$ \\
\hline 31 & 1 & -3.151667 & 1.457950 & -1.354163 \\
\hline 32 & 6 & -5.566382 & -0.574901 & -0.027674 \\
\hline 33 & 1 & -5.155999 & -2.324204 & 1.174390 \\
\hline 34 & 1 & -5.641840 & 1.204126 & -1.251915 \\
\hline 35 & 6 & -6.987750 & -0.741956 & ๑.016968 \\
\hline 36 & 6 & 7.168442 & -0.617298 & -0.143446 \\
\hline 37 & 7 & -8.145679 & -0.877887 & ๑. 056867 \\
\hline 38 & 7 & 8.327256 & -0.740535 & -0.185740 \\
\hline
\end{tabular}

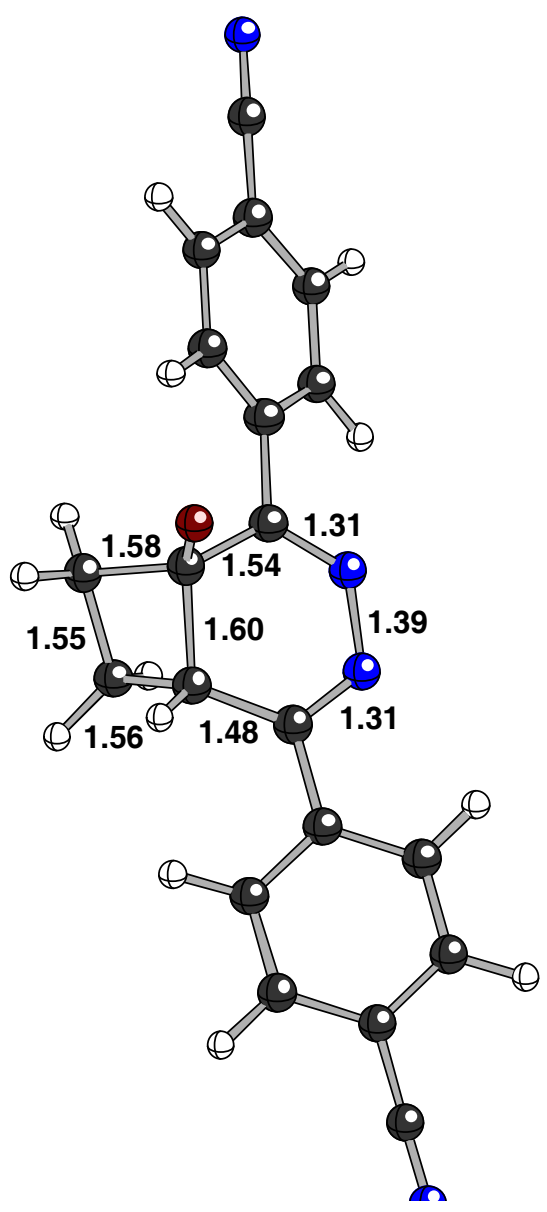




\section{bis-p-CN substituted Electrocyclic TS ( $n=1$, footnote 25$)$}

$\mathrm{HF}=-1064.190798$ hartrees $(-667790.36765298 \mathrm{kcal} / \mathrm{mol})$

Imaginary Frequencies: 1 (-286.4770 1/cm)

Zero-point correction $=0.279739$ (Hartree/Particle)

Temperature 298.150 Kelvin. Pressure 1.00000 Atm.

Sum of electronic and thermal Free Energies = -1063.961649 hartrees (-667646.57436399 $\mathrm{kcal} / \mathrm{mol})$

\begin{tabular}{|c|c|c|c|c|}
\hline \multirow{2}{*}{$\begin{array}{l}\text { Center } \\
\text { Number }\end{array}$} & \multirow{2}{*}{$\begin{array}{l}\text { Atomic } \\
\text { Number }\end{array}$} & \multicolumn{3}{|c|}{ Coordinates (Angstroms) } \\
\hline & & $\mathrm{x}$ & $\mathrm{Y}$ & z \\
\hline 1 & 6 & -0.477815 & 2.776201 & 1.118729 \\
\hline 2 & 1 & -0.461648 & 2.257591 & 2.084312 \\
\hline 3 & 1 & -1.103225 & 3.672440 & 1.192173 \\
\hline 4 & 6 & 0.918466 & 2.954938 & $\odot .536059$ \\
\hline 5 & 1 & 1.744898 & 3.075588 & 1.249657 \\
\hline 6 & 1 & 0.938080 & 3.796668 & -0.163252 \\
\hline 7 & 6 & -0.954697 & 1.849681 & -0.051563 \\
\hline 8 & 6 & -1.366058 & $\odot .470786$ & 0.413274 \\
\hline 9 & 6 & 0.957126 & 1.643858 & $-\odot .289939$ \\
\hline 10 & 1 & 1.291518 & 1.773299 & -1.323098 \\
\hline 11 & 6 & 1.449748 & 0.457559 & $\odot .370203$ \\
\hline 12 & 8 & -1.519209 & 2.406280 & -1.053078 \\
\hline 13 & 7 & -0.607362 & -0.344786 & 1.109741 \\
\hline 14 & 7 & 0.736232 & -0.285092 & 1.230537 \\
\hline 15 & 6 & 2.814706 & -0.052547 & $\odot .116471$ \\
\hline 16 & 6 & 3.230816 & -1.305338 & 0.626494 \\
\hline 17 & 6 & 3.757480 & 0.698820 & $-\odot .619908$ \\
\hline 18 & 6 & 4.519765 & -1.775166 & 0.424731 \\
\hline 19 & 1 & 2.506844 & -1.890651 & 1.181173 \\
\hline 20 & 6 & 5.043998 & 0.224651 & $-\odot .850522$ \\
\hline 21 & 1 & 3.478297 & 1.673977 & -1.003539 \\
\hline 22 & 6 & 5.444464 & -1.017907 & -0.325533 \\
\hline 23 & 1 & 4.821175 & -2.737224 & 0.828222 \\
\hline 24 & 1 & 5.750315 & 0.816371 & -1.424801 \\
\hline 25 & 6 & -2.727762 & -0.055816 & $\odot .136855$ \\
\hline 26 & 6 & -3.238327 & -1.115579 & 0.924796 \\
\hline 27 & 6 & -3.556988 & 0.476723 & -0.874246 \\
\hline 28 & 6 & -4.513514 & -1.618882 & 0.719493 \\
\hline 29 & 1 & -2.603970 & -1.527875 & 1.700541 \\
\hline 30 & 6 & -4.839745 & -0.024937 & -1.082180 \\
\hline 31 & 1 & -3.161277 & 1.286181 & -1.476392 \\
\hline 32 & 6 & -5.333633 & -1.078175 & -0.293317 \\
\hline 33 & 1 & -4.888464 & -2.429626 & 1.336969 \\
\hline 34 & 1 & -5.464646 & 0.396023 & -1.864175 \\
\hline 35 & 6 & -6.650183 & -1.596378 & -0.511620 \\
\hline 36 & 6 & 6.771198 & -1.505101 & -0.548833 \\
\hline 37 & 7 & -7.723316 & -2.019334 & -0.686311 \\
\hline 38 & 7 & 7.853232 & -1.901838 & $-\odot .729656$ \\
\hline
\end{tabular}

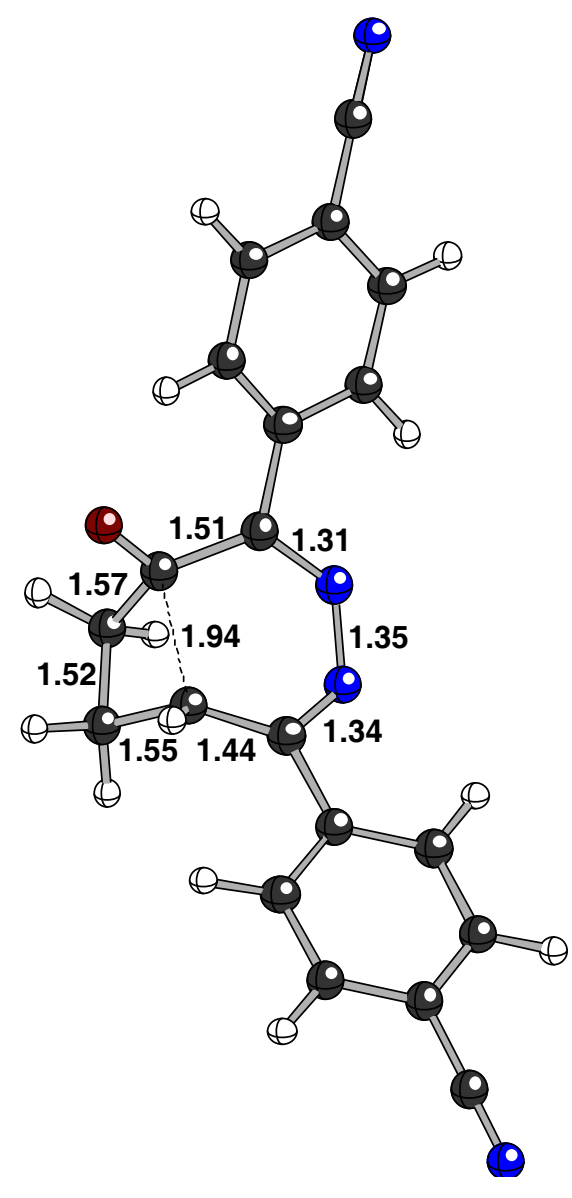




\section{bis- $p$-CN substituted Fragmentation TS ( $n=1$, footnote 25$)$}

$\mathrm{HF}=-1064.168484$ hartrees $(-667776.36539484 \mathrm{kcal} / \mathrm{mol})$

Imaginary Frequencies: 1 (-598.6094 1/cm)

Zero-point correction $=0.278871$ (Hartree/Particle)

Temperature 298.150 Kelvin. Pressure 1.00000 Atm.

Sum of electronic and thermal Free Energies = -1063.940024 hartrees (-667633.00446024 kcal/mol)

\begin{tabular}{|c|c|c|c|c|}
\hline \multirow{2}{*}{$\begin{array}{l}\text { Center } \\
\text { Number }\end{array}$} & \multirow{2}{*}{$\begin{array}{l}\text { Atomic } \\
\text { Number }\end{array}$} & \multicolumn{3}{|c|}{ Coordinates (Angstroms) } \\
\hline & & $\mathrm{X}$ & $\mathrm{Y}$ & Z \\
\hline 1 & 6 & -0.907434 & 1.708277 & -0.087592 \\
\hline 2 & 6 & -1.349617 & 0.308052 & -0.543008 \\
\hline 3 & 6 & 1.005103 & 1.735903 & -0.684249 \\
\hline 4 & 1 & $\odot .694036$ & 2.242026 & -1.588713 \\
\hline 5 & 6 & 1.462812 & 0.387675 & -0.822456 \\
\hline 6 & 8 & -1.555339 & 2.660925 & -0.613069 \\
\hline 7 & 7 & -0.657411 & -0.552706 & -1.248758 \\
\hline 8 & 7 & 0.715175 & -0.597483 & -1.322115 \\
\hline 9 & 6 & 2.825554 & -0.047818 & $-\odot .397008$ \\
\hline 10 & 6 & 3.101773 & -1.406301 & -0.141370 \\
\hline 11 & 6 & 3.882822 & 0.876220 & $-\odot .289834$ \\
\hline 12 & 6 & 4.378987 & -1.826738 & $\odot .209850$ \\
\hline 13 & 1 & 2.287716 & -2.116339 & $-\odot .235127$ \\
\hline 14 & 6 & 5.162344 & 0.471784 & 0.073699 \\
\hline 15 & 1 & 3.689509 & 1.919424 & $-\odot .518394$ \\
\hline 16 & 6 & 5.423152 & -0.888512 & ๑. 327812 \\
\hline 17 & 1 & 4.579332 & -2.876331 & 0.402735 \\
\hline 18 & 1 & 5.967630 & 1.195660 & 0.152556 \\
\hline 19 & 6 & -2.741973 & -0.091657 & -0.255134 \\
\hline 20 & 6 & -3.237826 & -1.352301 & $-\odot .668088$ \\
\hline 21 & 6 & -3.621117 & 0.758605 & 0.454013 \\
\hline 22 & 6 & -4.538508 & -1.742047 & -0.394369 \\
\hline 23 & 1 & -2.564816 & -2.008683 & -1.207385 \\
\hline 24 & 6 & -4.926037 & 0.370545 & 0.739032 \\
\hline 25 & 1 & -3.281078 & 1.742856 & 0.750845 \\
\hline 26 & 6 & -5.403832 & -0.884778 & 0.321133 \\
\hline 27 & 1 & -4.898802 & -2.713622 & -0.719547 \\
\hline 28 & 1 & -5.585944 & 1.040747 & 1.281630 \\
\hline 29 & 6 & 1.137280 & 2.523375 & $\odot .620030$ \\
\hline 30 & 6 & -0.169129 & 1.982430 & 1.253257 \\
\hline 31 & 1 & 0.035727 & 1.037143 & 1.776501 \\
\hline 32 & 1 & $-\odot .719894$ & 2.660730 & 1.921692 \\
\hline 33 & 1 & 2.034326 & 2.321544 & 1.217673 \\
\hline 34 & 1 & 1.074849 & 3.602101 & $\odot .445976$ \\
\hline 35 & 6 & -6.742948 & -1.289254 & 0.618497 \\
\hline 36 & 6 & 6.739968 & -1.313647 & 0.698594 \\
\hline 37 & 7 & -7.835323 & -1.621670 & ๑.859972 \\
\hline 38 & 7 & 7.812113 & -1.658645 & 1.000508 \\
\hline
\end{tabular}

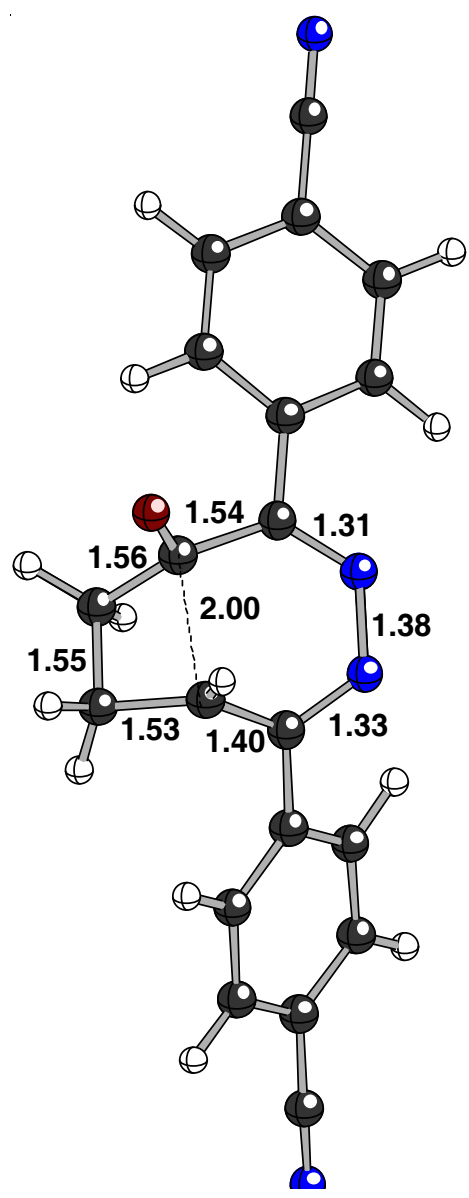




\section{bis-p-CN substituted Electrocyclic product $(n=1$, footnote 25$)$}

$\mathrm{HF}=-1064.2293092$ hartrees $(-667814.533816092 \mathrm{kcal} / \mathrm{mol})$

Imaginary Frequencies: none found

Zero-point correction $=0.281704$ (Hartree/Particle)

Temperature 298.150 Kelvin. Pressure 1.00000 Atm.

Sum of electronic and thermal Free Energies = -1063.999142 hartrees (-667670.10159642 $\mathrm{kcal} / \mathrm{mol})$

\begin{tabular}{|c|c|c|c|c|}
\hline \multirow{2}{*}{$\begin{array}{l}\text { Center } \\
\text { Number }\end{array}$} & \multirow{2}{*}{$\begin{array}{l}\text { Atomic } \\
\text { Number }\end{array}$} & \multicolumn{3}{|c|}{ Coordinates (Angstroms) } \\
\hline & & $\mathrm{X}$ & $\mathrm{Y}$ & Z \\
\hline 1 & 6 & -0.448680 & 3.506648 & ๑. 395962 \\
\hline 2 & 1 & -0.301539 & 3.297505 & 1.459124 \\
\hline 3 & 1 & -1.080302 & 4.396130 & 0.299544 \\
\hline 4 & 6 & 0.897790 & 3.759029 & -0.283122 \\
\hline 5 & 1 & 1.429915 & 4.595844 & 0.199302 \\
\hline 6 & 1 & 0.712103 & 4.076543 & -1.318960 \\
\hline 7 & 6 & -1.208024 & 2.377451 & $-\odot .315512$ \\
\hline 8 & 6 & -1.269931 & 1.083926 & ๑.338622 \\
\hline 9 & 6 & 1.777579 & 2.546945 & -0.311677 \\
\hline 10 & 1 & 2.618004 & 2.610187 & -1.001472 \\
\hline 11 & 6 & 1.655938 & 1.397846 & 0.391157 \\
\hline 12 & 8 & -1.847242 & 2.693424 & -1.340811 \\
\hline 13 & 7 & -0.457563 & $\odot .779386$ & 1.411689 \\
\hline 14 & 7 & 0.797719 & 1.033894 & 1.486227 \\
\hline 15 & 6 & 2.668663 & $\odot .313039$ & 0.184993 \\
\hline 16 & 6 & 2.285326 & -1.034209 & 0.331234 \\
\hline 17 & 6 & 4.010725 & 0.581040 & -0.146766 \\
\hline 18 & 6 & 3.187497 & -2.070529 & 0.116955 \\
\hline 19 & 1 & 1.263596 & -1.261285 & 0.612394 \\
\hline 20 & 6 & 4.925685 & -0.444653 & $-\odot .357821$ \\
\hline 21 & 1 & 4.349340 & 1.609225 & -0.219152 \\
\hline 22 & 6 & 4.520521 & -1.785966 & -0.231864 \\
\hline 23 & 1 & 2.868072 & -3.102815 & $0.21907 \odot$ \\
\hline 24 & 1 & 5.957754 & -0.216399 & -0.605301 \\
\hline 25 & 6 & -2.398410 & 0.168426 & 0.162794 \\
\hline 26 & 6 & $-2.55 \odot 252$ & -0.955501 & 1.024185 \\
\hline 27 & 6 & -3.380860 & 0.327562 & -0.852439 \\
\hline 28 & 6 & -3.614705 & -1.832844 & 0.905256 \\
\hline 29 & 1 & -1.810992 & -1.111279 & 1.800814 \\
\hline 30 & 6 & -4.441826 & -0.558587 & -0.979676 \\
\hline 31 & 1 & -3.285763 & 1.161077 & -1.535240 \\
\hline 32 & 6 & -4.585105 & -1.654850 & -0.105000 \\
\hline 33 & 1 & -3.703685 & -2.672578 & 1.588913 \\
\hline 34 & 1 & -5.174984 & -0.407500 & -1.767185 \\
\hline 35 & 6 & -5.674658 & -2.566027 & -0.242037 \\
\hline 36 & 6 & 5.461495 & -2.845357 & -0.443686 \\
\hline 37 & 7 & -6.568541 & -3.310674 & -0.350963 \\
\hline 38 & 7 & 6.231786 & -3.702947 & $-\odot .617626$ \\
\hline
\end{tabular}

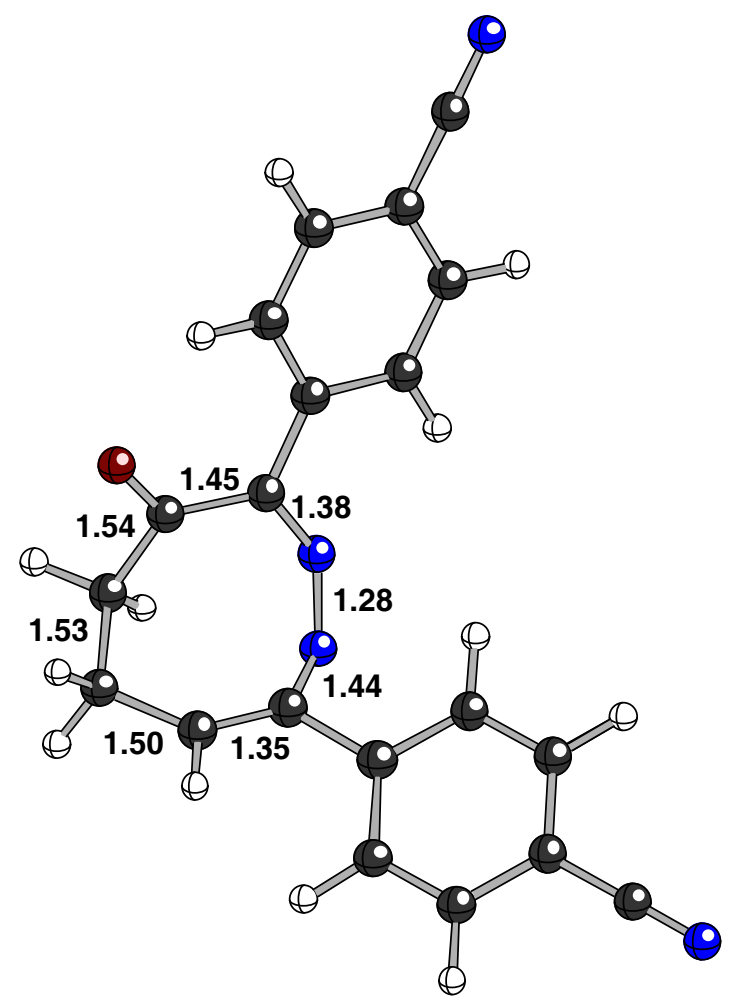




\section{bis-p-CN substituted Fragmentation product ( $n=1$, footnote 25$)$}

$\mathrm{HF}=-1064.1934842$ hartrees $(-667792.053270342 \mathrm{kcal} / \mathrm{mol})$

Imaginary Frequencies: none found

Zero-point correction $=0.281133$ (Hartree/Particle)

Temperature 298.150 Kelvin. Pressure 1.00000 Atm.

Sum of electronic and thermal Free Energies = -1063.963531 hartrees (-667647.75533781 $\mathrm{kcal} / \mathrm{mol})$

\begin{tabular}{|c|c|c|c|c|}
\hline \multirow{2}{*}{$\begin{array}{l}\text { Center } \\
\text { Number }\end{array}$} & \multirow{2}{*}{$\begin{array}{l}\text { Atomic } \\
\text { Number }\end{array}$} & \multicolumn{3}{|c|}{ Coordinates (Angstroms) } \\
\hline & & $\mathrm{x}$ & $\mathrm{Y}$ & z \\
\hline 1 & 6 & 0.920413 & 1.811865 & -0.499596 \\
\hline 2 & 6 & 1.271935 & $\odot .573202$ & $\odot .280767$ \\
\hline 3 & 6 & -1.278892 & 2.051333 & 1.045500 \\
\hline 4 & 1 & -0.404746 & 2.363994 & 1.617376 \\
\hline 5 & 6 & -1.560227 & 0.722379 & 1.141866 \\
\hline 6 & 8 & 1.769115 & 2.699437 & -0.657609 \\
\hline 7 & 7 & 0.607817 & -0.129649 & 1.232339 \\
\hline 8 & 7 & -0.606593 & -0.159967 & 1.713606 \\
\hline 9 & 6 & -2.777952 & 0.079839 & $\odot .614119$ \\
\hline 10 & 6 & -2.757081 & -1.278601 & $\odot .233575$ \\
\hline 11 & 6 & -4.004825 & 0.772557 & 0.529774 \\
\hline 12 & 6 & -3.900265 & -1.908665 & -0.245627 \\
\hline 13 & 1 & -1.825200 & -1.825104 & 0.327538 \\
\hline 14 & 6 & -5.150170 & 0.157338 & 0.042630 \\
\hline 15 & 1 & -4.051491 & 1.799230 & 0.878674 \\
\hline 16 & 6 & -5.108661 & -1.194749 & -0.354629 \\
\hline 17 & 1 & -3.867642 & -2.952962 & -0.540528 \\
\hline 18 & 1 & -6.085861 & 0.704642 & -0.018078 \\
\hline 19 & 6 & 2.663083 & $\odot .078004$ & $\odot .120238$ \\
\hline 20 & 6 & 3.201298 & -0.873949 & 1.026915 \\
\hline 21 & 6 & 3.504213 & 0.453870 & -0.959626 \\
\hline 22 & 6 & 4.471549 & -1.405870 & 0.873056 \\
\hline 23 & 1 & 2.580196 & -1.185854 & 1.857221 \\
\hline 24 & 6 & 4.774902 & -0.078736 & -1.124450 \\
\hline 25 & 1 & 3.160844 & 1.186626 & -1.677891 \\
\hline 26 & 6 & 5.288935 & -1.019758 & -0.210136 \\
\hline 27 & 1 & 4.847010 & -2.125692 & 1.595110 \\
\hline 28 & 1 & 5.383592 & 0.233331 & -1.968396 \\
\hline 29 & 6 & -1.441149 & 2.812264 & $-\odot .23864 \odot$ \\
\hline 30 & 6 & -0.439584 & 2.052798 & -1.176994 \\
\hline 31 & 1 & -0.907639 & 1.098524 & -1.440591 \\
\hline 32 & 1 & -0.236468 & 2.623459 & -2.091961 \\
\hline 33 & 1 & -2.440505 & 2.776525 & -0.682612 \\
\hline 34 & 1 & -1.149068 & 3.862600 & $-\odot .135287$ \\
\hline 35 & 6 & 6.598165 & -1.563320 & -0.374513 \\
\hline 36 & 6 & -6.289449 & -1.836794 & -0.848516 \\
\hline 37 & 7 & 7.669413 & -2.009519 & -0.510017 \\
\hline 38 & 7 & -7.252546 & -2.355845 & -1.252210 \\
\hline
\end{tabular}

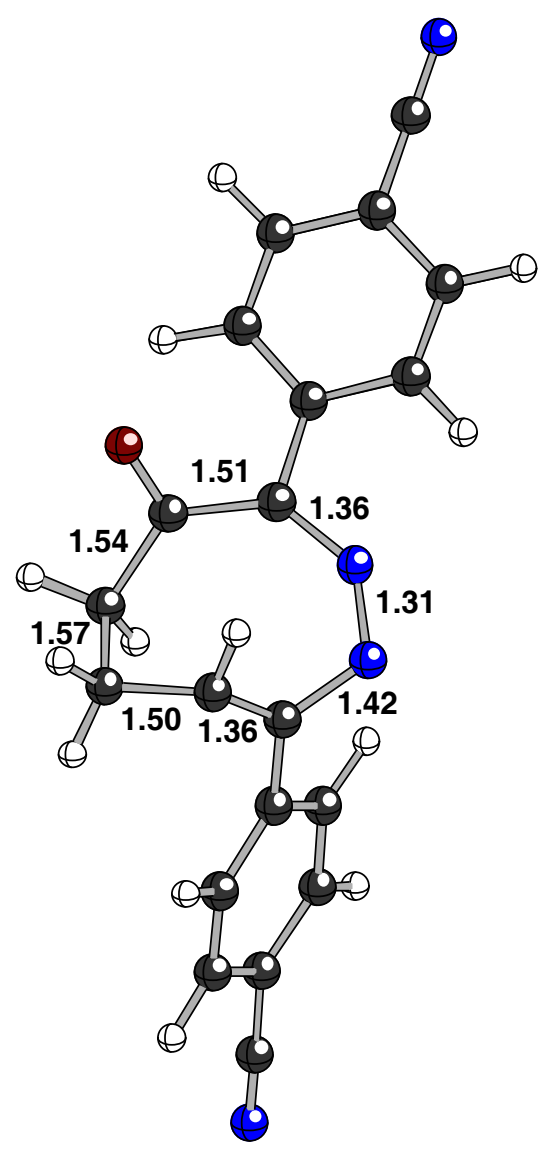




\section{di-CN substituted reactant ( $n=1$, footnote 25$)$}

$\mathrm{HF}=-602.0511437 \mathrm{hartrees}(-377793.113183187 \mathrm{kcal} / \mathrm{mol})$

Imaginary Frequencies: none found

Zero-point correction $=0.119574$ (Hartree/Particle)

Temperature 298.150 Kelvin. Pressure 1.00000 Atm.

Sum of electronic and thermal Free Energies $=\mathbf{- 6 0 1 . 9 6 8 3 4 2}$ hartrees $(-377741.15428842 \mathrm{kcal} / \mathrm{mol})$

\begin{tabular}{|c|c|c|c|c|}
\hline \multirow{2}{*}{$\begin{array}{l}\text { Center } \\
\text { Number }\end{array}$} & \multirow{2}{*}{$\begin{array}{l}\text { Atomic } \\
\text { Number }\end{array}$} & \multicolumn{3}{|c|}{ Coordinates (Angstroms) } \\
\hline & & $\mathrm{X}$ & $\mathrm{Y}$ & Z \\
\hline 1 & 6 & 0.996332 & 1.930963 & $-\odot .425939$ \\
\hline 2 & 1 & 1.903511 & 1.898892 & -1.039848 \\
\hline 3 & 1 & 0.995204 & 2.848427 & 0.173016 \\
\hline 4 & 6 & -0.368514 & 1.683026 & -1.110809 \\
\hline 5 & 1 & -0.285366 & 1.042060 & -1.993734 \\
\hline 6 & 1 & -0.982168 & 2.551813 & -1.366936 \\
\hline 7 & 6 & 0.705103 & 0.719046 & 0.532896 \\
\hline 8 & 6 & 1.139082 & -0.623417 & -0.067842 \\
\hline 9 & 6 & -0.841088 & 0.875447 & 0.144262 \\
\hline 10 & 1 & -1.350643 & 1.527796 & 0.860357 \\
\hline 11 & 6 & -1.546208 & -0.413912 & -0.031514 \\
\hline 12 & 8 & 1.105805 & 0.658987 & 1.781961 \\
\hline 13 & 7 & 0.398224 & -1.668436 & -0.347181 \\
\hline 14 & 7 & -0.984190 & -1.560952 & -0.307551 \\
\hline 15 & 6 & 2.563323 & -0.854736 & -0.122891 \\
\hline 16 & 6 & -2.981732 & -0.447688 & 0.104644 \\
\hline 17 & 7 & 3.709877 & -0.996617 & -0.276411 \\
\hline 18 & 7 & -4.141734 & -0.395889 & 0.213230 \\
\hline
\end{tabular}




\section{di-CN substituted Electrocyclic TS ( $n=1$, footnote 25$)$}

$\mathrm{HF}=-602.0485234$ hartrees $(-377791.468918734 \mathrm{kcal} / \mathrm{mol})$

Imaginary Frequencies: 1 (-306.8685 1/cm)

Zero-point correction $=0.118635$ (Hartree/Particle)

Temperature 298.150 Kelvin. Pressure 1.00000 Atm.

Sum of electronic and thermal Free Energies = -601.966182 hartrees $(-377739.79886682 \mathrm{kcal} / \mathrm{mol})$

\begin{tabular}{|c|c|c|c|c|}
\hline \multirow{2}{*}{$\begin{array}{l}\text { Center } \\
\text { Number }\end{array}$} & \multirow{2}{*}{$\begin{array}{l}\text { Atomic } \\
\text { Number }\end{array}$} & \multicolumn{3}{|c|}{ Coordinates (Angstroms) } \\
\hline & & $\mathrm{x}$ & $\mathrm{Y}$ & Z \\
\hline 1 & 6 & 1.061791 & 1.892650 & -0.439014 \\
\hline 2 & 1 & 2.041076 & 1.871370 & -0.933643 \\
\hline 3 & 1 & $\odot .953588$ & 2.838739 & 0.102467 \\
\hline 4 & 6 & -0.194482 & 1.575419 & -1.265961 \\
\hline 5 & 1 & -0.002442 & $\odot .842007$ & -2.054074 \\
\hline 6 & 1 & -0.721264 & 2.424978 & -1.713946 \\
\hline 7 & 6 & 0.770497 & 0.744627 & 0.580876 \\
\hline 8 & 6 & 1.117785 & -0.611103 & -0.058159 \\
\hline 9 & 6 & -0.939349 & 0.943781 & -0.074336 \\
\hline 10 & 1 & -1.482780 & 1.677584 & $\odot .522170$ \\
\hline 11 & 6 & -1.582863 & -0.339456 & -0.161331 \\
\hline 12 & 8 & 1.024960 & 0.829229 & 1.822805 \\
\hline 13 & 7 & 0.356142 & -1.554357 & -0.563886 \\
\hline 14 & 7 & -1.012175 & -1.479975 & -0.511956 \\
\hline 15 & 6 & 2.512587 & -0.967224 & 0.025996 \\
\hline 16 & 6 & -2.969234 & -0.456055 & 0.235396 \\
\hline 17 & 7 & 3.656771 & -1.194118 & 0.023533 \\
\hline 18 & 7 & -4.093346 & -0.483599 & 0.542849 \\
\hline
\end{tabular}

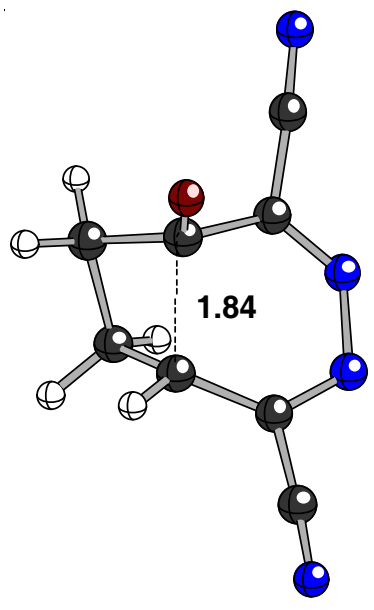




\section{di-CN substituted Fragmentation TS ( $\mathrm{n}=\mathbf{1}$, footnote 25$)$}

$\mathrm{HF}=-602.0161501$ hartrees $(-377771.154349251 \mathrm{kcal} / \mathrm{mol})$

Imaginary Frequencies: 1 (-629.2900 1/cm)

Zero-point correction $=0.117120$ (Hartree/Particle)

Temperature 298.150 Kelvin. Pressure 1.00000 Atm.

Sum of electronic and thermal Free Energies = -601.936012 hartrees $(-377720.86689012 \mathrm{kcal} / \mathrm{mol})$

\begin{tabular}{|c|c|c|c|c|}
\hline \multirow{2}{*}{$\begin{array}{l}\text { Center } \\
\text { Number }\end{array}$} & \multirow{2}{*}{$\begin{array}{l}\text { Atomic } \\
\text { Number }\end{array}$} & \multicolumn{3}{|c|}{ Coordinates (Angstroms) } \\
\hline & & $\mathrm{x}$ & $\mathrm{Y}$ & Z \\
\hline 1 & 6 & -0.872710 & $\odot .905175$ & -0.088625 \\
\hline 2 & 6 & -1.319469 & -0.578081 & -0.023756 \\
\hline 3 & 6 & 0.976141 & 0.676280 & $-0.80140 \odot$ \\
\hline 4 & 1 & 0.655433 & 0.780233 & -1.830443 \\
\hline 5 & 6 & 1.418670 & -0.614186 & -0.380011 \\
\hline 6 & 8 & -1.589286 & 1.596840 & -0.859911 \\
\hline 7 & 7 & -0.674295 & -1.662248 & -0.374304 \\
\hline 8 & 7 & 0.706469 & -1.731893 & $-\odot .371926$ \\
\hline 9 & 6 & 1.208735 & 1.891193 & $\odot .089147$ \\
\hline 10 & 6 & -0.027412 & 1.630544 & 0.992410 \\
\hline 11 & 1 & 0.241402 & 0.931433 & 1.796970 \\
\hline 12 & 1 & -0.537361 & 2.505589 & 1.418884 \\
\hline 13 & 1 & 2.167695 & 1.911377 & 0.622567 \\
\hline 14 & 1 & 1.101580 & 2.824083 & -0.471073 \\
\hline 15 & 6 & -2.697045 & -0.787432 & 0.324109 \\
\hline 16 & 6 & 2.754232 & -0.761639 & 0.184430 \\
\hline 17 & 7 & -3.810441 & -0.911720 & 0.652128 \\
\hline 18 & 7 & 3.840937 & -0.822505 & 0.603326 \\
\hline
\end{tabular}

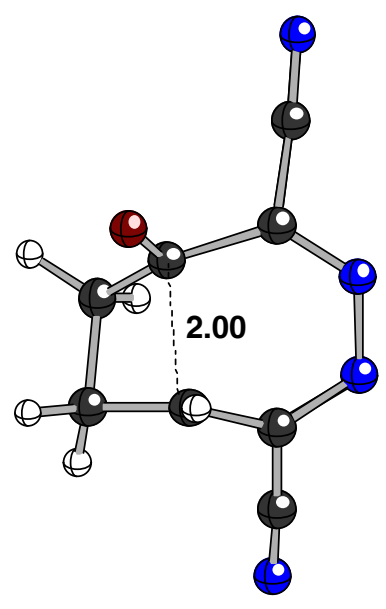




\section{di-CN substituted Electrocyclic product $(n=1$, footnote 25$)$}

$\mathrm{HF}=-602.0825249$ hartrees $(-377812.805199999 \mathrm{kcal} / \mathrm{mol})$

Imaginary Frequencies: none found

Zero-point correction $=0.119965$ (Hartree/Particle)

Temperature 298.150 Kelvin. Pressure 1.00000 Atm.

Sum of electronic and thermal Free Energies = -602.000406 hartrees $(-377761.27476906 \mathrm{kcal} / \mathrm{mol})$

\begin{tabular}{|c|c|c|c|c|}
\hline \multirow{2}{*}{$\begin{array}{l}\text { Center } \\
\text { Number }\end{array}$} & \multirow{2}{*}{$\begin{array}{l}\text { Atomic } \\
\text { Number }\end{array}$} & \multicolumn{3}{|c|}{ Coordinates (Angstroms) } \\
\hline & & $\mathrm{x}$ & $\mathrm{Y}$ & Z \\
\hline 1 & 6 & 0.601492 & 2.050946 & -0.065354 \\
\hline 2 & 1 & 1.425637 & 2.582761 & -0.563730 \\
\hline 3 & 1 & 0.273271 & 2.694884 & $\odot .757467$ \\
\hline 4 & 6 & -0.551712 & 1.796776 & -1.042466 \\
\hline 5 & 1 & -0.206361 & 1.171142 & -1.873723 \\
\hline 6 & 1 & -0.889783 & 2.749814 & -1.467557 \\
\hline 7 & 6 & 1.265975 & 0.812912 & 0.550454 \\
\hline 8 & 6 & 1.226264 & -0.509786 & -0.120222 \\
\hline 9 & 6 & -1.676158 & 1.122072 & -0.322956 \\
\hline 10 & 1 & -2.442691 & 1.738986 & $\odot .141832$ \\
\hline 11 & 6 & -1.758777 & -0.223666 & -0.191309 \\
\hline 12 & 8 & 2.047256 & 1.017628 & 1.485786 \\
\hline 13 & 7 & 0.311496 & -1.260767 & -0.787366 \\
\hline 14 & 7 & -0.979123 & -1.226418 & -0.804260 \\
\hline 15 & 6 & 2.498496 & -1.180618 & -0.087052 \\
\hline 16 & 6 & -2.908699 & -0.765595 & 0.500724 \\
\hline 17 & 7 & 3.541243 & -1.708354 & $-\odot .078409$ \\
\hline 18 & 7 & -3.833531 & -1.189726 & 1.068394 \\
\hline
\end{tabular}




\section{di-CN substituted Fragmentation product $(n=1$, footnote 25$)$}

$\mathrm{HF}=-602.0525229$ hartrees $(-377793.978644979 \mathrm{kcal} / \mathrm{mol})$

Imaginary Frequencies: none found

Zero-point correction $=0.119885$ (Hartree/Particle)

Temperature 298.150 Kelvin. Pressure 1.00000 Atm.

Sum of electronic and thermal Free Energies = -601.970105 hartrees $(-377742.26058855 \mathrm{kcal} / \mathrm{mol})$

\begin{tabular}{|c|c|c|c|c|}
\hline \multirow{2}{*}{$\begin{array}{l}\text { Center } \\
\text { Number }\end{array}$} & \multirow{2}{*}{$\begin{array}{l}\text { Atomic } \\
\text { Number }\end{array}$} & \multicolumn{3}{|c|}{ Coordinates (Angstroms) } \\
\hline & & $\mathrm{x}$ & $\mathrm{Y}$ & Z \\
\hline 1 & 6 & 1.018028 & 1.032916 & -0.240947 \\
\hline 2 & 6 & 1.246550 & $-\odot .433357$ & $-\odot .044041$ \\
\hline 3 & 6 & -1.246306 & 0.382699 & 1.242787 \\
\hline 4 & 1 & -0.467892 & 0.226583 & 1.987826 \\
\hline 5 & 6 & -1.529276 & -0.701545 & 0.489155 \\
\hline 6 & 8 & 2.015408 & 1.768194 & -0.299746 \\
\hline 7 & 7 & $\odot .569283$ & -1.585131 & 0.273804 \\
\hline 8 & 7 & -0.676178 & -1.848801 & $\odot .517984$ \\
\hline 9 & 6 & -1.353722 & 1.767812 & 0.697897 \\
\hline 10 & 6 & -0.337940 & 1.732892 & $-\odot .495975$ \\
\hline 11 & 1 & -0.843189 & 1.216888 & -1.320237 \\
\hline 12 & 1 & -0.082314 & 2.745630 & -0.823649 \\
\hline 13 & 1 & -2.347844 & 2.020668 & 0.312057 \\
\hline 14 & 1 & -1.068883 & 2.523252 & 1.437297 \\
\hline 15 & 6 & 2.649556 & -0.758045 & -0.165016 \\
\hline 16 & 6 & -2.582900 & -0.710807 & -0.486310 \\
\hline 17 & 7 & 3.772439 & -1.063267 & -0.266199 \\
\hline 18 & 7 & -3.450843 & -0.753367 & -1.265678 \\
\hline
\end{tabular}

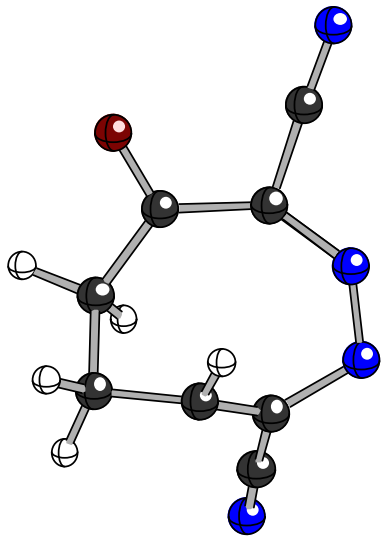




\section{IRC Diagrams}

Structures on the following IRC plots correspond to the transition state structures (center images) and to the last structures from the forward and reverse IRC calculations (side images).

\section{$\underline{3^{\prime} \rightarrow 4 \text { (Figure 1) }}$}

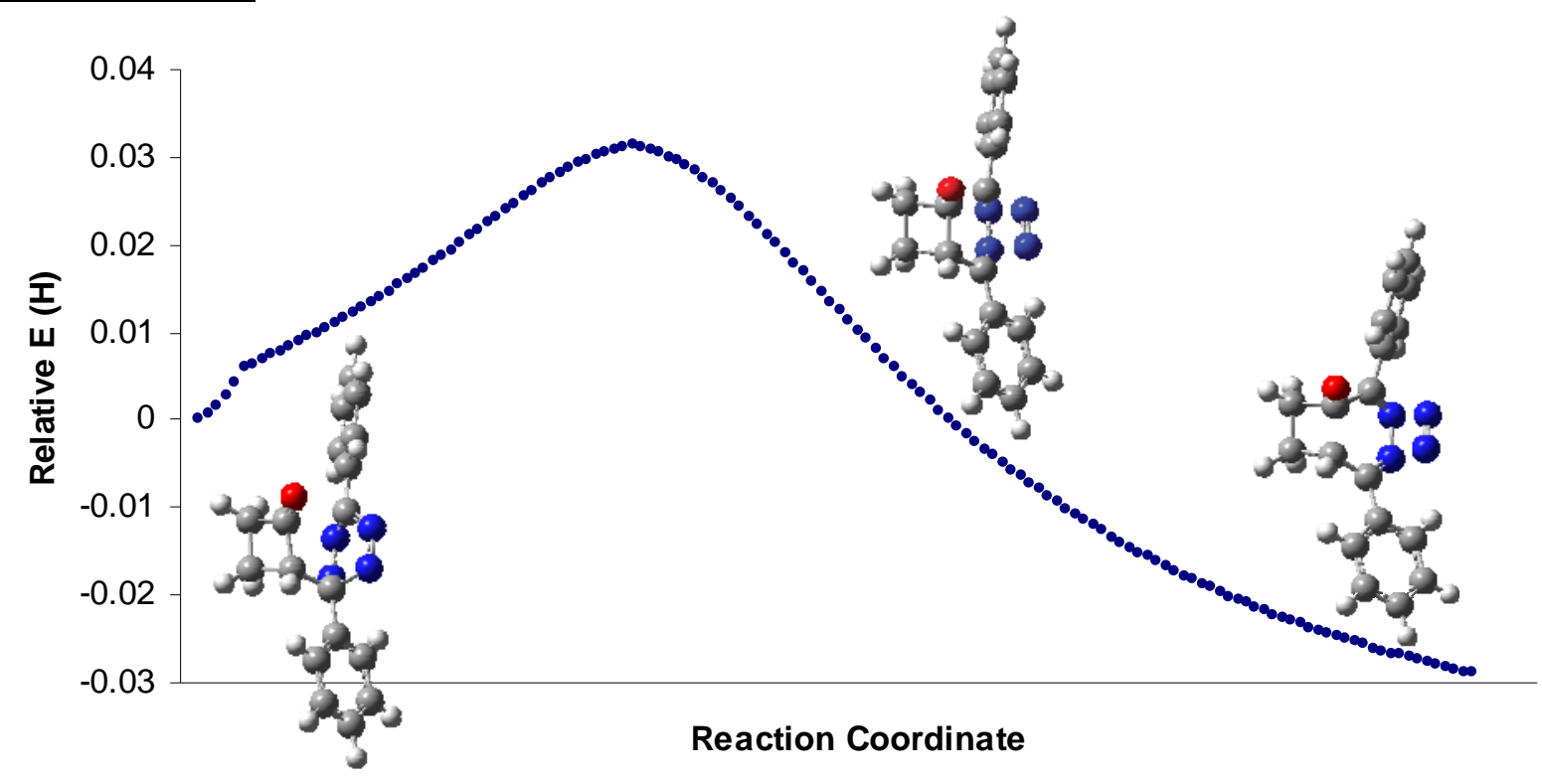

$\underline{4 \rightarrow 6}$ (Figure 2) / cyclobutanone allowed electrocyclic reaction (Table 2)

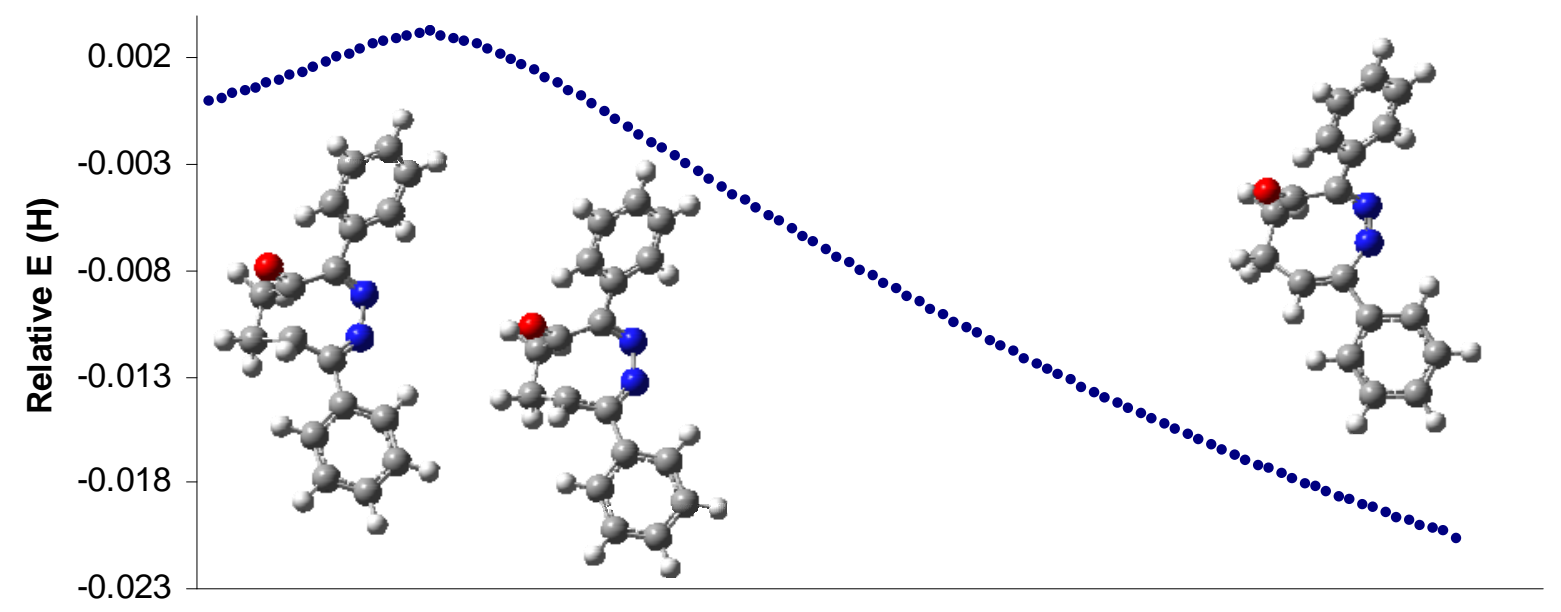

Reaction Coordinate 
$\underline{4 \rightarrow 7 \text { (Figure 2) / cyclobutanone fragmentation reaction (Table 2) }}$

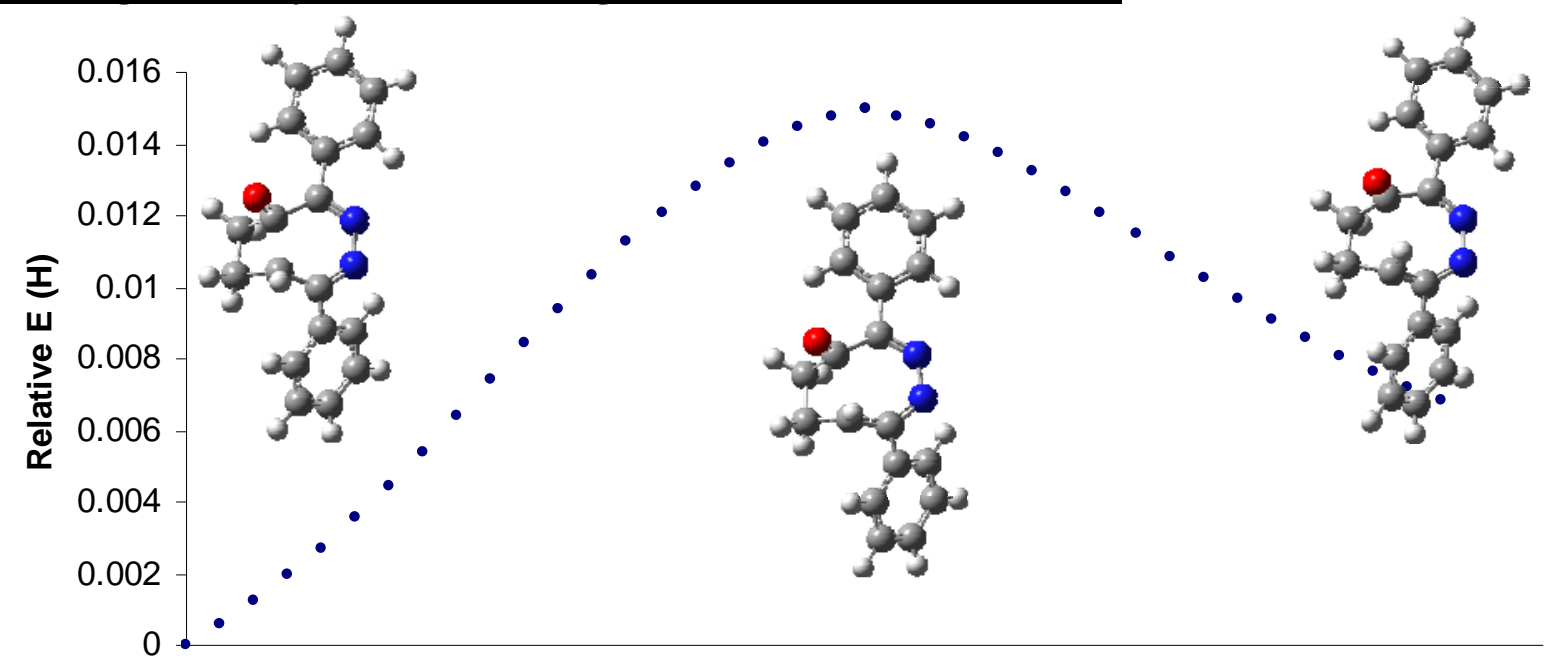

Reaction Coordinate

Cyclopentanone allowed electrocyclic reaction (Table 2)

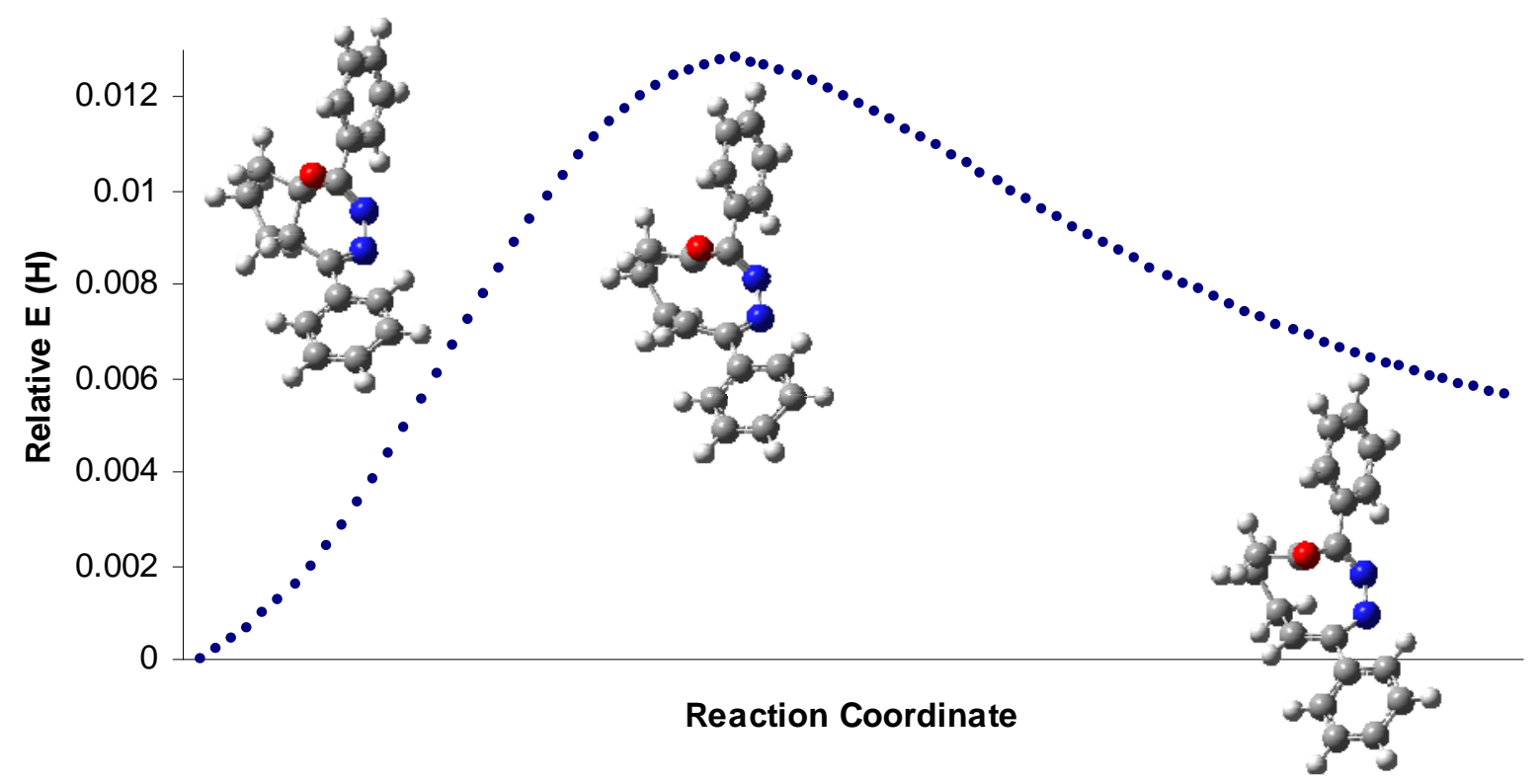




\section{Cyclopentanone fragmentation reaction (Table 2)}

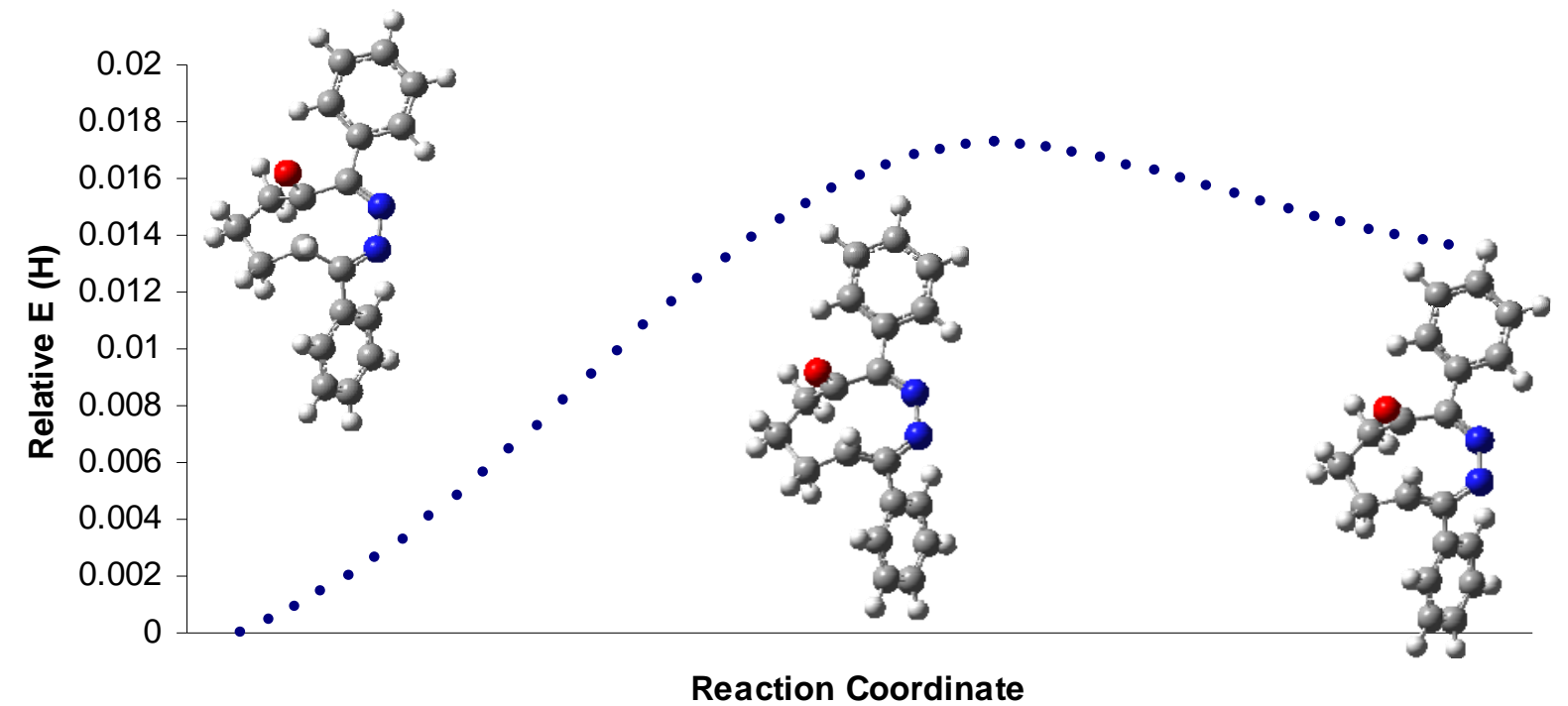

Cyclohexanone 1,2-shift reaction (Table 2)

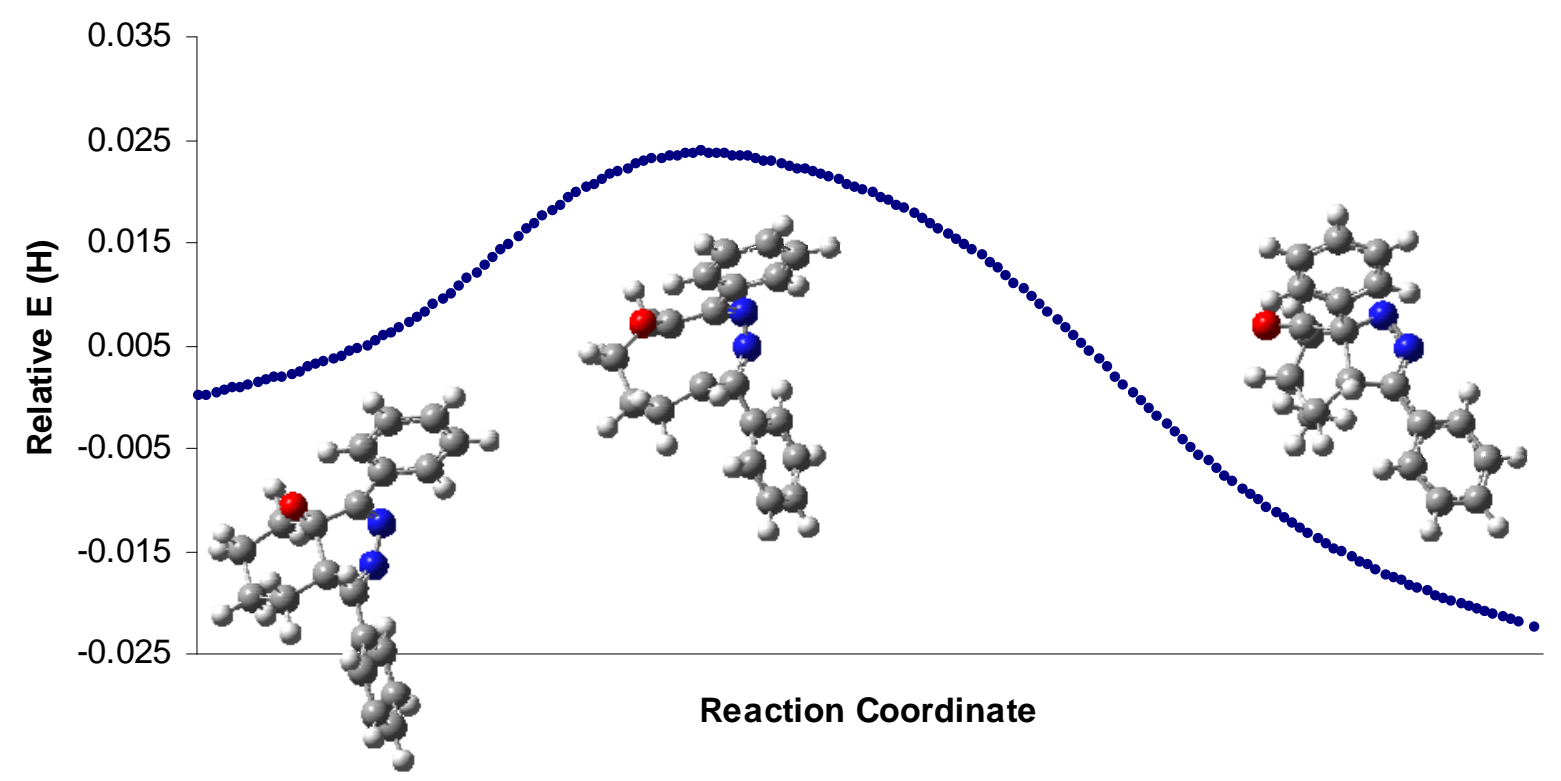




\section{Cyclohexanone fragmentation reaction (Table 2)}

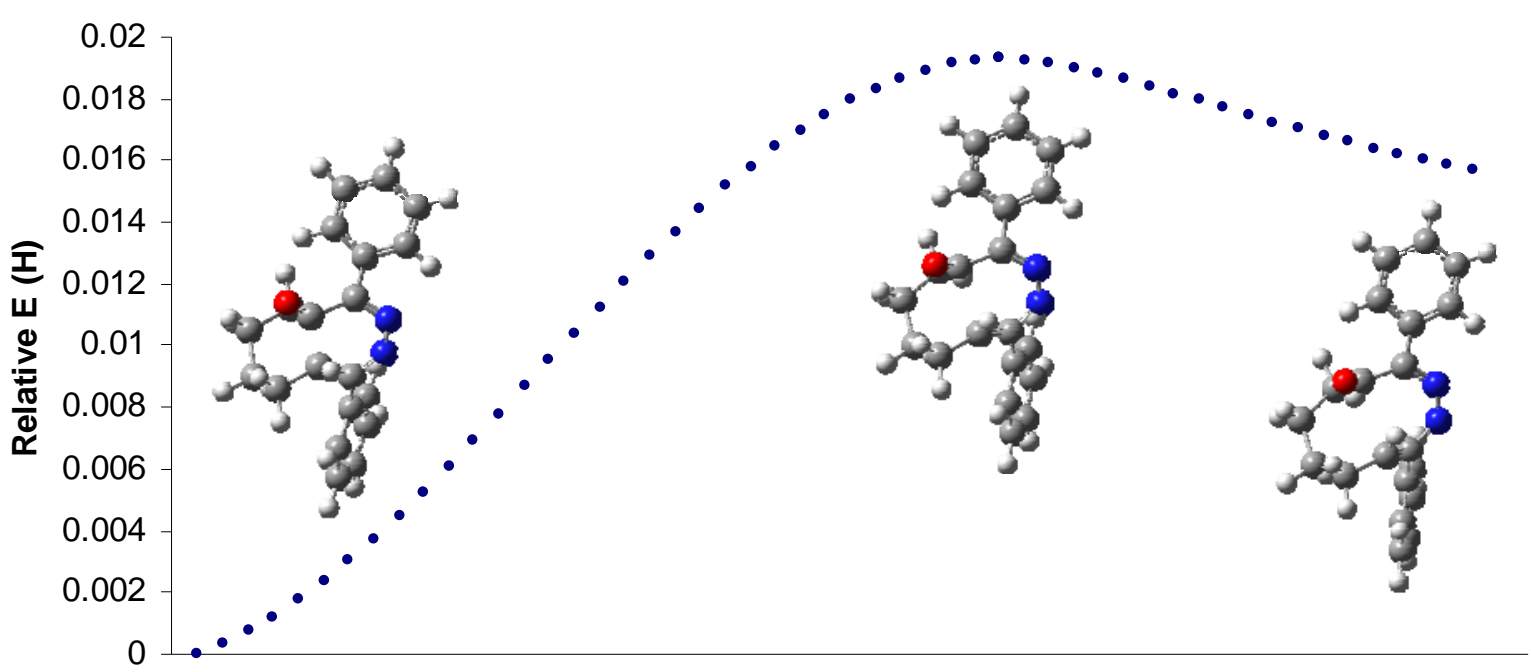

Reaction Coordinate

\section{Cycloheptanone 1,2-shift reaction (Table 2)}

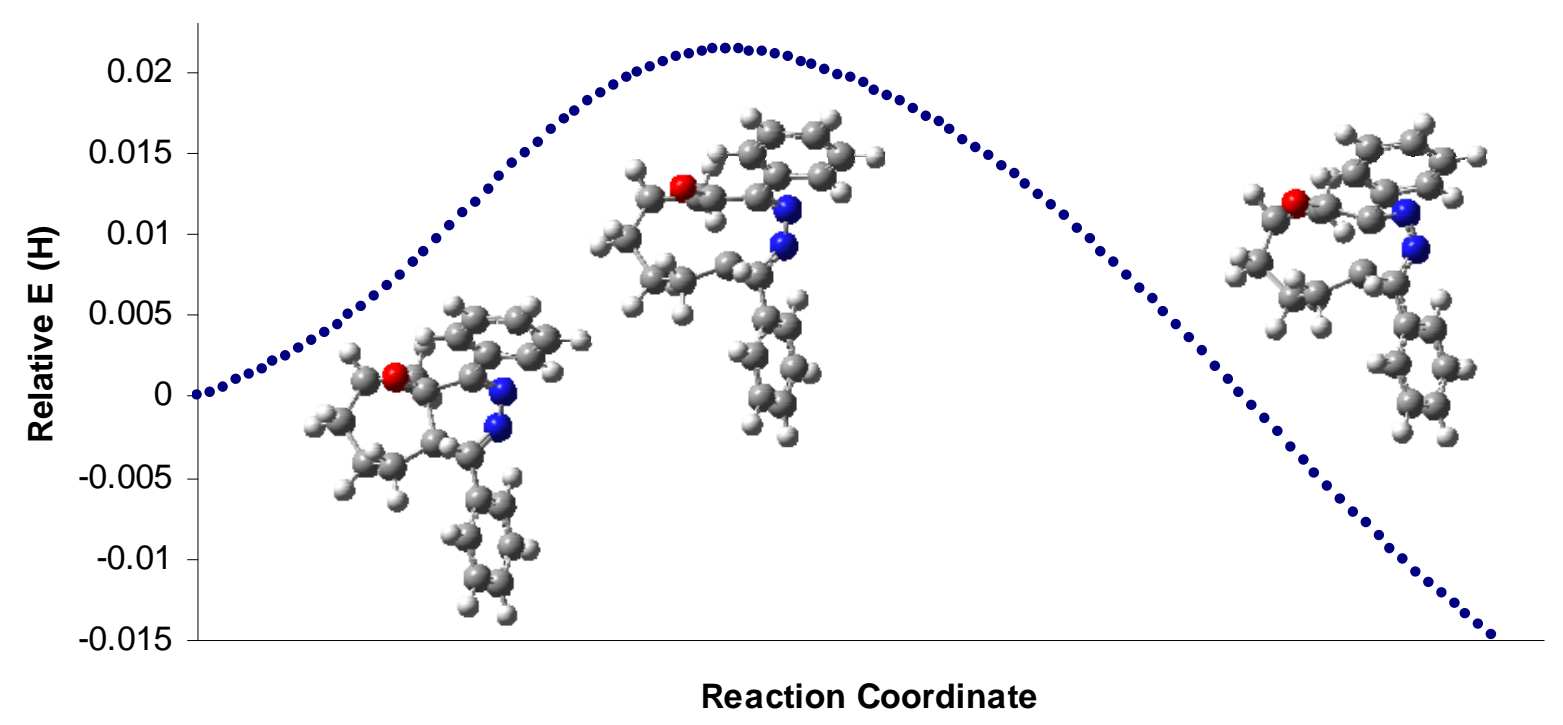




\section{Cycloheptanone fragmentation reaction (Table 2)}

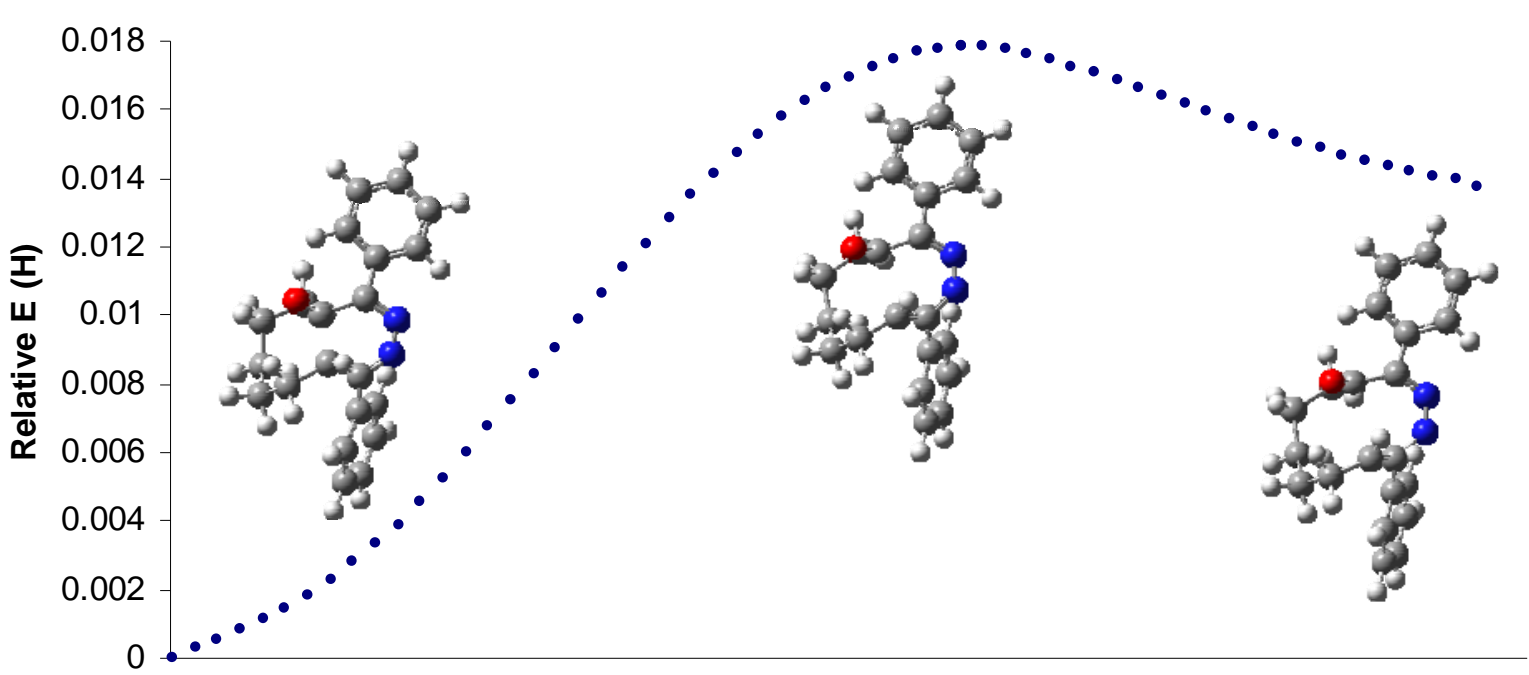

Reaction Coordinate

\section{Cyclooctanone allowed electrocyclic reaction (Table 2)}

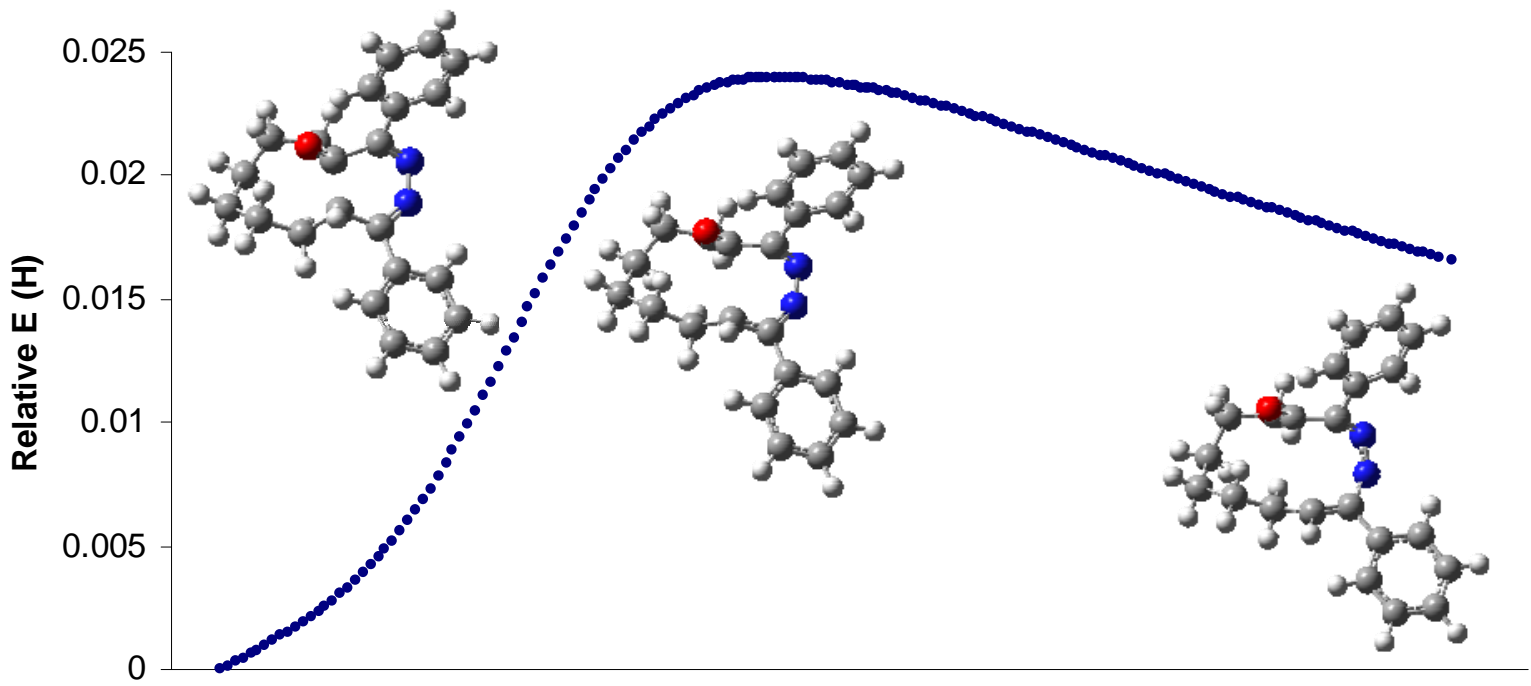

Reaction Coordinate 


\section{Cyclooctonone fragmentation reaction (Table 2)}

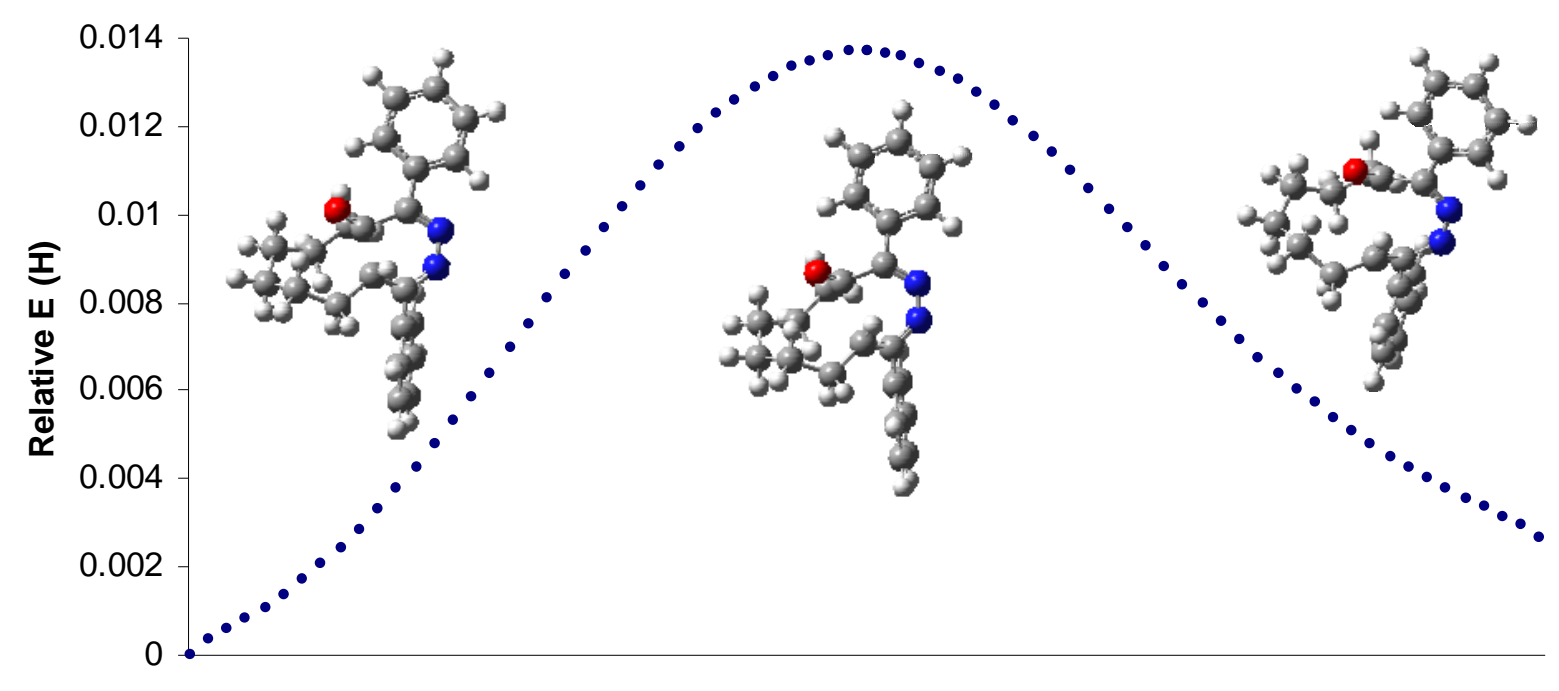

Reaction Coordinate

$\underline{12 \rightarrow \text { pyridazine ( } n=1 \text {, pyridazine figure and scheme in SI and footnote } 26)}$

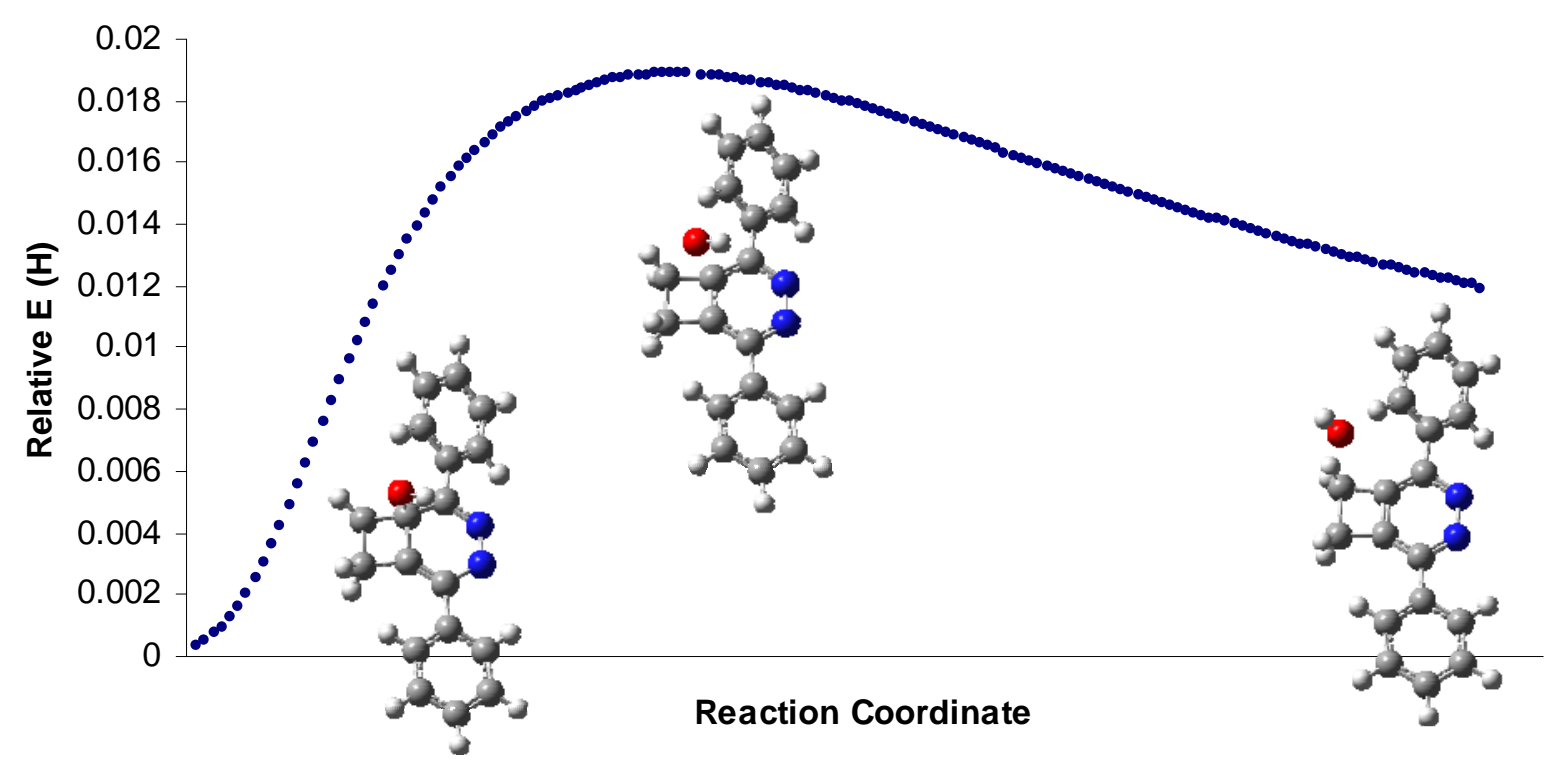


$\underline{12 \rightarrow \text { pyridazine ( } n=2 \text {, pyridazine figure and scheme in SI and footnote 26) }}$

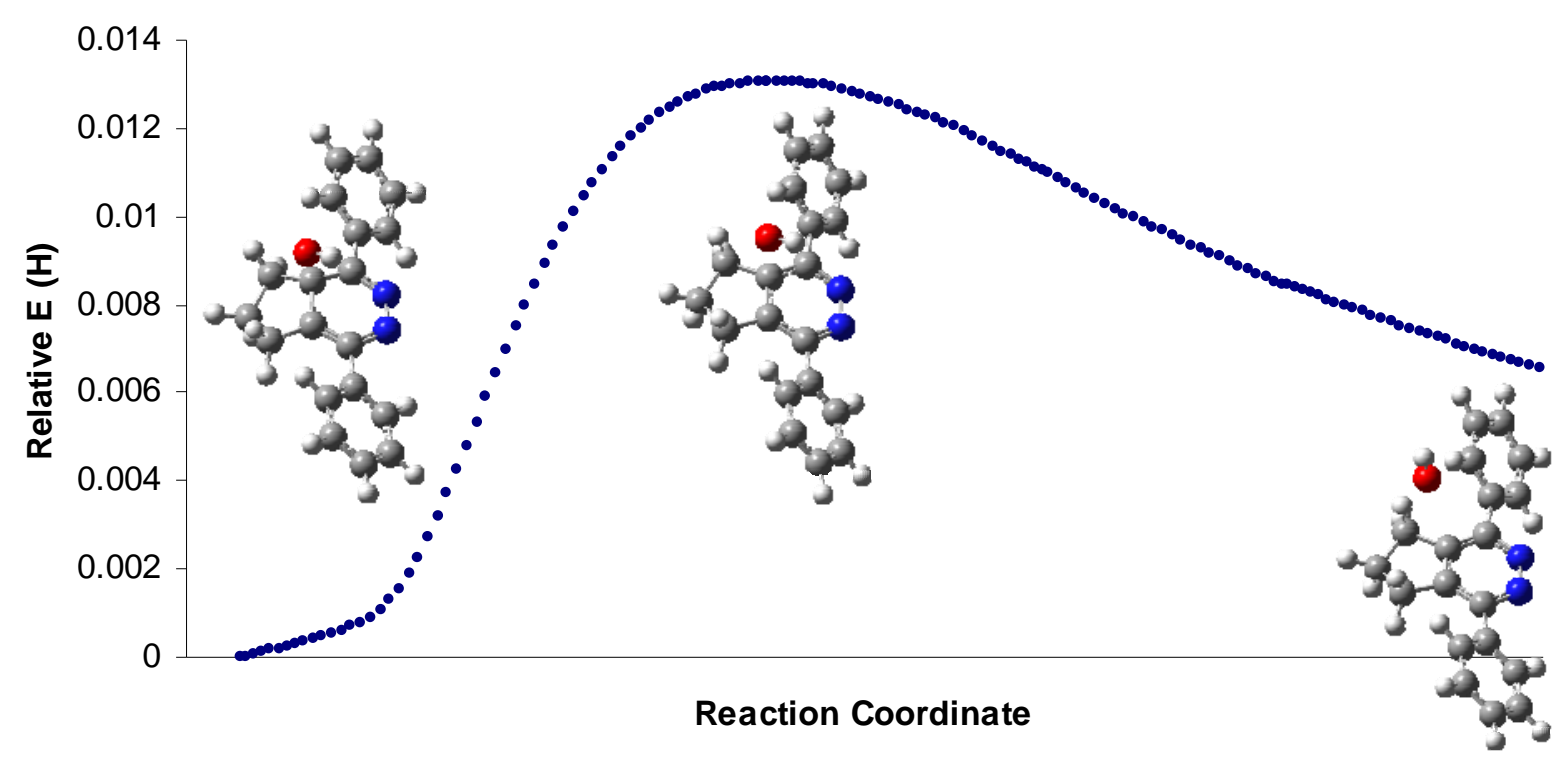

